\title{
High-Order Deterministic Sensitivity Analysis and Uncertainty Quantification: Review and New Developments
}

\author{
Dan Gabriel Cacuci
}

Citation: Cacuci, D.G. High-Order Deterministic Sensitivity Analysis and Uncertainty Quantification: Review and New Developments. Energies 2021, 14, 6715. https: //doi.org/10.3390/en14206715

Academic Editors: Victor Becerra and José Matas

Received: 20 August 2021

Accepted: 7 October 2021

Published: 15 October 2021

Publisher's Note: MDPI stays neutral with regard to jurisdictional claims in published maps and institutional affiliations.

Copyright: (C) 2021 by the author. Licensee MDPI, Basel, Switzerland. This article is an open access article distributed under the terms and conditions of the Creative Commons Attribution (CC BY) license (https:/ / creativecommons.org/licenses/by/ $4.0 /)$.
Center for Nuclear Science and Energy, Department of Mechanical Engineering, University of South Carolina, Columbia, SC 29208, USA; cacuci@cec.sc.edu

\begin{abstract}
This work reviews the state-of-the-art methodologies for the deterministic sensitivity analysis of nonlinear systems and deterministic quantification of uncertainties induced in model responses by uncertainties in the model parameters. The need for computing high-order sensitivities is underscored by presenting an analytically solvable model of neutron scattering in a hydrogenous medium, for which all of the response's relative sensitivities have the same absolute value of unity. It is shown that the wider the distribution of model parameters, the higher the order of sensitivities needed to achieve a desired level of accuracy in representing the response and in computing the response's expectation, variance, skewness and kurtosis. This work also presents new mathematical expressions that extend to the sixth-order of the current state-of-the-art fourth-order formulas for computing fourth-order correlations among computed model response and model parameters. Another novelty presented in this work is the mathematical framework of the 3rd-Order Comprehensive Adjoint Sensitivity Analysis Methodology for Nonlinear Systems (3rd-CASAM-N), which enables the most efficient computation of the exact expressions of the 1st-, 2nd- and 3rd-order functional derivatives ("sensitivities") of a model's response to the underlying model parameters, including imprecisely known initial, boundary and/or interface conditions. The 2nd- and 3rd-level adjoint functions are computed using the same forward and adjoint computer solvers as used for solving the original forward and adjoint systems. Comparisons between the CPU times are also presented for an OECD/NEA reactor physics benchmark, highlighting the fact that finite-difference schemes would not only provide approximate values for the respective sensitivities (in contradistinction to the 3rd-CASAM-N, which provides exact expressions for the sensitivities) but would simply be unfeasible for computing sensitivities of an order higher than first-order. Ongoing work will generalize the 3rd-CASAM-N to a higher order while aiming to overcome the curse of dimensionality.
\end{abstract}

Keywords: six-order moments of model response distribution in parameter phase space; third-order comprehensive adjoint sensitivity analysis methodology for nonlinear systems

\section{Introduction}

The functional derivatives of the results produced by models with respect to the underlying model parameters are customarily called the model "response sensitivities". The various methods for computing first-order sensitivities can be grouped into two broad categories: statistical methods and deterministic methods. There is a plethora of statistical methods, but all are subject to the "curse of dimensionality" [1], in that the number of computations increases exponentially with the number of parameters and order of sensitivity. Additionally, none of the statistical methods can compute sensitivities exactly. On the other hand, there are just three deterministic methods for computing sensitivities: (i) finite-differences, which can produce approximate results within the order of approximation of the respective finite-difference formulas and are also subject to the curse of dimensionality; (ii) the forward variational method, which obtains exact expressions for the sensitivities but is also subject to the curse of dimensionality, and (iii) the adjoint 
sensitivity analysis method, which also produces exact expressions for the sensitivities but can overcome the curse of dimensionality, as will be discussed in this work.

The field of nuclear science and engineering has provided the earliest use [2] of adjoint operators for performing a sensitivity analysis of a large-scale linear system, modeled by the linear Boltzmann transport equation and comprising many model parameters. The large system analyzed by Wigner [2] was the model of linear neutron transport through the core of a nuclear reactor. The reactor's multiplication factor was the "response", and the various neutron cross-sections influencing the reactor's behavior were the "model parameters". Wigner's ideas were generalized and set in a rigorous mathematical (functional analysis) framework for generic nonlinear systems by Cacuci [3,4]. Several works [5-9] in the field of nuclear engineering pioneered the computation of specific 2 nd-order sensitivities of reaction rates and/or multiplication factors for nuclear reactors modeled by forward and adjoint linear Boltzmann or diffusion equations. These works generally indicated that, for a system comprising TP model parameters, the computation of 2 nd-order sensitivities of responses to model parameters required $O\left(T P^{2}\right)$ large-scale computations per response. Therefore, only the 2nd-order sensitivities that were deemed a priori to be "important" were actually computed, and these 2nd-order sensitivities turned out to be considerably smaller than the corresponding 1st-order sensitivities. These findings likely gave rise to the generally held opinion that "2nd-order sensitivities are generally insignificant in reactor physics". This opinion was likely responsible for the diminishing interest in computing 2nd-order response sensitivities, which practically vanished in the nuclear engineering field in the 1990s. On the other hand, interest in computing 2nd-order sensitivities became evident in other fields, such as structural mechanics [10-15], parametric circuit analysis and optimization [16,17] and the atmospheric and earth sciences; see, e.g., References [18-22].

All of the 2nd-order adjoint methods mentioned above were developed for specific applications for which they usually estimated rather than computed exactly and exhaustively as 2nd-order response sensitivities to the model's parameters. Cacuci [23-25] conceived the generally applicable "Second-Order Adjoint Sensitivity Analysis Methodology" for both linear [23] and nonlinear [24,25] systems, which enables the most efficient computation of the exact expressions of all of the second-order functional derivatives of model responses to model parameters while overcoming the curse of dimensionality. This methodology has been applied [26-31] to compute the exact expressions of the first- and second-order sensitivities to the parameters of the leakage response of an OECD/NEA reactor physics benchmark [32]. This OECD/NEA benchmark was modeled using the neutron transport code PARTISN [33]. As has been shown in References [26-31], this reactor physics benchmark comprises 21,976 model parameters, which give rise to 21,976 first-order sensitivities (of which 7477 have nonzero values) and 241,483,276 second-order sensitivities (of which $27,956,503$ have nonzero values).

The 2nd-order methodology developed in References [23-25] has been extended by Cacuci $[34,35]$ to a 3rd order and has been applied [36-38] to perform a 3rd-order sensitivity and uncertainty analysis of the OECD/NEA benchmark [32]. Recently, Cacuci [39] developed the "Fourth-Order Comprehensive Adjoint Sensitivity Analysis (4th-CASAM) of Response-Coupled Linear Forward/Adjoint Systems", which has been applied to perform [40,41] an initial 4th-order sensitivity analysis of the OECD/NEA benchmark [32].

As is well-known, model responses of linear systems may involve the solutions of both the forward and the adjoint linear models that correspond to the respective physical ones. Since nonlinear operators do not admit bona fide adjoint operators (only a linearized form of a nonlinear operator admits an adjoint operator), the responses of nonlinear models can depend only on the forward functions. Hence, linear systems cannot be treated as particular cases of nonlinear systems but need to be treated separately; for this reason, the adjoint sensitivity analysis methodology for linear systems will not be reviewed in this work.

The mathematical formulation of the equations that underlies the computational model of a generic nonlinear physical system will be presented in Section 2, along with 
the definition of a generic response that depends on the model's state variables and parameters, which are considered to be uncertain (i.e., not known precisely). Besides the initial conditions and correlations, the model parameters are also considered to include geometrical parameters that describe the system's boundaries and internal interfaces. Section 3 presents the sixth-order Taylor series expansion of a model's response in the model's uncertain parameters. Section 3 also illustrates the need for computing highorder sensitivities by means of a paradigm physical model of neutron scattering in an infinite hydrogenous medium, which admits a closed form solution for all orders of the sensitivities of the model's response (neutron flux) to the model's scattering cross-section. This model was deliberately chosen, because the relative sensitivities of all orders of the model's response with respect to the scattering cross-section are unity. The sixth-order Taylor series expansion presented in Section 3 provides the basis for the new formulas, accurate up to and including the sixth order in the parameters' standard deviations, for the quantification of uncertainties induced in the model's response by the uncertainties in the model. These new formulas are presented in Section 4, along with additional new formulas that provide the expressions for the correlations up to the fourth order among the model responses and model parameters. By means of a paradigm physical model of neutron scattering in an infinite hydrogenous medium, Section 4 also illustrates the need for high-order uncertainty quantification.

Section 5 presents the novel Third-Order Comprehensive Adjoint Sensitivity Analysis Methodology for Nonlinear Systems (3rd-CASAM-N). Section 5 also presents illustrative comparisons of the CPU times needed by the 3rd-CASAM versus finite-difference formulas for computing sensitivities of various orders for the OECD/NEA benchmark [32]. These comparisons underscore the fact that finite-difference schemes would not only provide approximate values for the respective sensitivities (in contradistinction to the 3rd-CASAM$\mathrm{N}$, which provides exact expressions for the sensitivities) but would simply be unfeasible for computing sensitivities of an order higher than first-order. Since statistical methods require orders of magnitude more computational time and resources than finite-differences, it is evident that the statistical methods could not even compute the first-order sensitivities of the benchmark's response to the benchmark's 21,976 model parameters.

The concluding discussion presented in Section 5 highlights the fact that the 3rdCASAM-N presented in this work provides a fundamentally important step in the quest to overcome the "curse of dimensionality" in the sensitivity analysis, uncertainty quantification and predictive modeling.

\section{Mathematical Description of a Generic Nonlinear Physical System with Uncertain Parameters and Boundaries}

The modeling of a physical system and/or the result of an indirect experimental measurement includes the following modeling components:

(a) A mathematical model comprising independent variables (e.g., space, time, etc.); dependent variables (aka "state functions", e.g., temperature, mass, momentum, etc.) and various parameters (appearing in correlations, coordinates of physical boundaries, etc.), which are all interrelated by equations (linear and/or nonlinear in the state functions) that usually represent conservation laws.

(b) Model parameters, which usually stem from processes that are external to the system under consideration and are seldom, if ever, known precisely. The known characteristics of the model parameters may include their nominal (expected/mean) values and, possibly, higher-order moments or cumulants (i.e., variance/covariances, skewness and kurtosis), which are usually determined from experimental data and/or processes external to the physical system under consideration. Occasionally, only inequality and/or equality constraints that delimit the ranges of the system's parameters are known. 
(c) One or several computational results, customarily called "system responses" (or objective functions or indices of performance), which are computed using the mathematical model.

(d) Experimentally measured values of the responses under consideration, which may be used to infer nominal (expected) values and uncertainties (variances, covariances, skewness, kurtosis, etc.) of the respective measured responses.

Without loss of generality, the imprecisely known model parameters can be considered to be real-valued scalar quantities. These model parameters will be denoted as $\alpha_{1}, \ldots$, $\alpha_{T P}$, where TP denotes the "total number of imprecisely known parameters" underlying the model under consideration. For subsequent developments, it is convenient to consider that these parameters are components of a "vector of parameters" denoted as $\alpha \triangleq\left(\alpha_{1}, \ldots, \alpha_{T P}\right)^{\dagger} \in \mathrm{E}_{\alpha} \in \mathbb{R}^{T P}$, where $\mathrm{E}_{\alpha}$ is also a normed linear space and where $\mathbb{R}^{T P}$ denotes the TP-dimensional subset of the set of real scalars. The components of the $T P$-dimensional column vector $\alpha \in \mathbb{R}^{T P}$ are considered to include imprecisely known geometrical parameters that characterize the physical system's boundaries in the phase-space of the model's independent variables. Matrices will be denoted using capital bold letters while vectors will be denoted using either capital or lower-case bold letters. The symbol "ڤ" will be used to denote "is defined as" or "is by definition equal to". Transposition will be indicated by a dagger $(\dagger)$ superscript.

The model is considered to comprise TI independent variables which will be denoted as $x_{i}, i=1, \ldots, T I$, and which are considered to be components of a TI-dimensional column vector denoted as $\mathbf{x} \triangleq\left(x_{1}, \ldots, x_{T I}\right)^{\dagger} \in \mathbb{R}^{T I}$, where the sub/superscript " $T I^{\prime}$ " denotes the "Total (number of) Independent variables". The vector $\mathbf{x} \in \mathbb{R}^{T I}$ of independent variables is considered to be defined on a phase-space domain which will be denoted as $\Omega(\boldsymbol{\alpha})$ and which is defined as follows: $\Omega(\boldsymbol{\alpha}) \triangleq\left\{-\infty \leq \lambda_{i}(\boldsymbol{\alpha}) \leq x_{i} \leq \omega_{i}(\boldsymbol{\alpha}) \leq \infty ; i=1, \ldots, T I\right\}$. The lower boundary-point of an independent variable is denoted as $\lambda_{i}(\boldsymbol{\alpha})$ and the corresponding upper boundary-point is denoted as $\omega_{i}(\boldsymbol{\alpha})$. A typical example of boundaries that depend on both geometrical parameters and material properties are the "boundaries facing vacuum" in models based on diffusion theory, where conditions are imposed on the "extrapolated boundary" of the respective spatial domain. The "extrapolated boundary" depends both on the imprecisely known physical dimensions of the problem's domain and also on the medium's properties, such as atomic number densities and microscopic transport cross sections. The boundary of $\Omega(\boldsymbol{\alpha})$, which will be denoted as $\partial \Omega(\boldsymbol{\alpha})$, comprises the set of all of the endpoints $\lambda_{i}(\boldsymbol{\alpha}), \omega_{i}(\boldsymbol{\alpha}), i=1, \ldots, T I$, of the respective intervals on which the components of $\mathbf{x}$ are defined, i.e., $\partial \Omega(\boldsymbol{\alpha}) \triangleq\left\{\lambda_{i}(\boldsymbol{\alpha}) \cup \omega_{i}(\boldsymbol{\alpha}), i=1, \ldots, T I\right\}$.

A nonlinear physical system can be generally represented/modeled by means of coupled equations which can be represented in operator form as follows:

$$
\mathbf{N}[\mathbf{u}(\mathbf{x}), \boldsymbol{\alpha}]=\mathbf{Q}(\mathbf{x}, \boldsymbol{\alpha}), \mathbf{x} \in \Omega_{x}(\boldsymbol{\alpha}) .
$$

The quantities which appear in Equation (1) are defined as follows:

1. $\quad \mathbf{u}(\mathbf{x}) \triangleq\left[u_{1}(\mathbf{x}), \ldots, u_{T D}(\mathbf{x})\right]^{\dagger}$ is a $T D$-dimensional column vector of dependent variables; the abbreviation "TD" denotes "Total (number of) Dependent variables." The functions $u_{i}(\mathbf{x}), i=1, \ldots, T D$, denote the system's "dependent variables" (also called "state functions"); $\mathbf{u}(\mathbf{x}) \in \mathrm{E}_{u}$, where $\mathrm{E}_{u}$ is a normed linear space over the scalar field $\mathrm{F}$ of real numbers.

2. $\mathbf{N}[\mathbf{u}(\mathbf{x}), \boldsymbol{\alpha}] \triangleq\left[N_{1}(\mathbf{u}, \boldsymbol{\alpha}), \ldots, N_{T D}(\mathbf{u}, \boldsymbol{\alpha})\right]^{\dagger}$ denotes a $T D$-dimensional column vector The components $N_{i}(\mathbf{u}, \boldsymbol{\alpha}), i=1, \ldots, T D$ are operators (including differential, difference, integral, distributions, and/or infinite matrices) acting (usually) nonlinearly on the dependent variables $\mathbf{u}(\mathbf{x})$, the independent variables $\mathbf{x}$ and the model parameters $\boldsymbol{\alpha} ; \mathbf{N}(\boldsymbol{\alpha}, \mathbf{u})$ is the mapping $\mathbf{N}: \mathrm{D} \subset \mathrm{E} \rightarrow \mathrm{E}_{Q}$, where $\mathrm{D}=\mathrm{D}_{u} \oplus \mathrm{D}_{\alpha}, \mathrm{D}_{u} \subset \mathrm{E}_{u}$, $\mathrm{D}_{\alpha} \subset \mathrm{E}_{\alpha}, \mathrm{E}=\mathrm{E}_{u} \oplus \mathrm{E}_{\alpha}$. An arbitrary element $\mathbf{e} \in \mathrm{E}$ has the form $\mathbf{e}=(\boldsymbol{\alpha}, \mathbf{u})$. 
3. $\mathbf{Q}(\mathbf{x}, \boldsymbol{\alpha}) \triangleq\left[q_{1}(\mathbf{x} ; \boldsymbol{\alpha}), \ldots, q_{T D}(\mathbf{x} ; \boldsymbol{\alpha})\right]^{\dagger}$ is a $T D$-dimensional column vector which represents inhomogeneous source terms, which usually depend nonlinearly on the uncertain parameters $\alpha ; \mathbf{Q} \in \mathrm{E}_{\mathrm{Q}}$, where $\mathrm{E}_{Q}$ is also a normed linear space.

4. The equalities in this work are considered to hold in the weak ("distributional") sense. The right-sides of Equation (1) and of other various equations to be derived in this work may contain "generalized functions/functionals", particularly Diracdistributions and derivatives thereof.

Boundary and/or initial conditions must also be provided if differential operators appear in Equation (1). In an operator form, these boundary and/or initial conditions are represented as follows:

$$
\mathbf{B}[\mathbf{u}(\mathbf{x}), \mathbf{x} ; \boldsymbol{\alpha}]-\mathbf{C}(\mathbf{x}, \boldsymbol{\alpha})=0, \mathbf{x} \in \partial \Omega_{x}(\boldsymbol{\alpha}) .
$$

In Equation (2), the components $B_{i}(\mathbf{u} ; \boldsymbol{\alpha}), i=1, \ldots, T D$ of $\mathbf{B}(\mathbf{u}, \boldsymbol{\alpha}) \triangleq\left[B_{1}(\mathbf{u} ; \boldsymbol{\alpha}), \ldots, B_{T D}\right.$ $(\mathbf{u} ; \boldsymbol{\alpha})]^{\dagger}$ are nonlinear operators of $\mathbf{u}(\mathbf{x})$ and $\boldsymbol{\alpha}$, which are defined on the boundary $\partial \Omega_{x}(\boldsymbol{\alpha})$ of the model's domain $\Omega_{x}(\boldsymbol{\alpha})$. The components $C_{i}(\mathbf{u} ; \boldsymbol{\alpha}), i=1, \ldots, T D$ of $\mathbf{C}(\mathbf{x}, \boldsymbol{\alpha}) \triangleq$ $\left[C_{1}(\mathbf{u} ; \boldsymbol{\alpha}), \ldots, C_{T D}(\mathbf{u} ; \boldsymbol{\alpha})\right]^{\dagger}$ comprise inhomogeneous boundary sources which are nonlinear functions of $\alpha$.

Solving Equations (1) and (2) at the nominal parameter values $\alpha^{0} \triangleq\left[\alpha_{1}^{0}, \ldots, \alpha_{i}^{0}, \ldots, \alpha_{T P}^{0}\right]^{\dagger}$ provides "the nominal solution" $\mathbf{u}^{0}(\mathbf{x})$, i.e., the vectors $\mathbf{u}^{0}(\mathbf{x})$ and $\alpha^{0}$ satisfy the following equations:

$$
\begin{gathered}
\mathbf{N}\left[\mathbf{u}^{0}(\mathbf{x}), \boldsymbol{\alpha}^{0}\right]=\mathbf{Q}\left(\mathbf{x}, \boldsymbol{\alpha}^{0}\right), \mathbf{x} \in \Omega_{x}, \\
\mathbf{B}\left[\mathbf{u}^{0}(\mathbf{x}), \mathbf{x} ; \boldsymbol{\alpha}^{0}\right]-\mathrm{C}\left(\mathbf{x}, \boldsymbol{\alpha}^{0}\right)=0, \quad \mathbf{x} \in \partial \Omega_{x}\left(\boldsymbol{\alpha}^{0}\right) .
\end{gathered}
$$

Responses of particularly important interest are model representations/computations of physical measurements of the model's state function $\mathbf{u}\left(\mathbf{x}_{p}\right)$ at a specific point, $\mathbf{x}_{p}$, in phase-space. Other responses of particular interest are averages over the phase-space domain (or segments thereof). Both point-measurements and "average" responses can be represented generically in the following integral form:

$$
\begin{aligned}
& R[\mathbf{u}(\mathbf{x}), \boldsymbol{\alpha} ; \mathbf{x}] \triangleq \prod_{i=1}^{T I} \int_{\lambda_{i}(\boldsymbol{\alpha})}^{\omega_{i}(\boldsymbol{\alpha})} S[\mathbf{u}(\mathbf{x}) ; \boldsymbol{\alpha}] d x_{i} \\
& \prod_{i=1}^{T I} \int_{\lambda_{i}(\boldsymbol{\alpha})}^{\omega_{i}(\boldsymbol{\alpha})}[] d x_{i} \triangleq \int_{\lambda_{1}(\boldsymbol{\alpha})}^{\omega_{1}(\boldsymbol{\alpha})} \ldots \int_{\lambda_{i}(\boldsymbol{\alpha})}^{\omega_{i}(\boldsymbol{\alpha})} \ldots \int_{\lambda_{T I}(\boldsymbol{\alpha})}^{\omega_{T I}(\boldsymbol{\alpha})}[] d x_{1} d x_{2} \ldots d x_{i} \ldots d x_{T I},
\end{aligned}
$$

where $S[\mathbf{u}(\mathbf{x}) ; \boldsymbol{\alpha}]$ is suitably differentiable nonlinear function of $\mathbf{u}(\mathbf{x})$ and of $\boldsymbol{\alpha}$. It is important to note that the components of $\alpha$ also include parameters that may appear specifically just in the definition of the response under consideration, but which might not appear in Equations (1) and (2). In particular, a measurement of a physical quantity can be represented as a response $R_{p}\left[\boldsymbol{\varphi}\left(\mathbf{x}_{p}\right), \boldsymbol{\psi}\left(\mathbf{x}_{p}\right) ; \boldsymbol{\alpha}\right]$ located at a point, $\mathbf{x}_{p}$, which may itself be afflicted by uncertainties. Such a response can be represented mathematically in the form $R_{p}\left[\mathbf{u}\left(\mathbf{x}_{p}\right) ; \boldsymbol{\alpha}\right] \triangleq \prod_{i=1}^{T I} \int_{\lambda_{i}(\boldsymbol{\alpha})}^{\omega_{i}(\boldsymbol{\alpha})} R_{p}[\mathbf{u}(\mathbf{x}) ; \boldsymbol{\alpha}] \delta\left(\mathbf{x}-\mathbf{x}_{p}\right) d x_{i}$, where $\delta\left(\mathbf{x}-\mathbf{x}_{p}\right)$ denotes the multidimensional Dirac-delta functional. The measurement point $\mathbf{x}_{p}$ appears only in the definition of the response but does not appear in Equations (1) and (2). Thus, the (physical) "system" includes not only the computational model but also the model's response.

\section{Sixth-Order Formulas for Sensitivity Analysis of Model Responses to Model Parameters}

The system's response is a function of the system's parameters $\alpha \triangleq\left(\alpha_{1}, \ldots, \alpha_{T P}\right)^{\dagger} \in$ $\mathbb{R}^{T P}$, either directly or indirectly through the state functions. Up to 6th-order, the explicit 
Taylor-series of a system response, denoted as $R_{k}(\boldsymbol{\alpha})$, around the expected (or nominal) parameter values $\alpha^{0}$ has the following well-known formal expression:

$$
\begin{gathered}
R_{k}^{(0)}(\boldsymbol{\alpha})=R_{k}\left(\boldsymbol{\alpha}^{0}\right), \\
R_{k}^{(1)}(\boldsymbol{\alpha})=R_{k}^{(0)}(\boldsymbol{\alpha})+\sum_{j 1=1}^{T P} \frac{\partial R_{k}\left(\boldsymbol{\alpha}^{0}\right)}{\partial \alpha_{j 1}}\left(\alpha_{j 1}-\alpha_{j 1}^{0}\right), \\
R_{k}^{(2)}(\boldsymbol{\alpha})=R_{k}^{(1)}(\boldsymbol{\alpha})+\frac{1}{2} \sum_{j 1=1}^{T P} \sum_{j 2=1}^{T P} \frac{\partial^{2} R_{k}\left(\boldsymbol{\alpha}^{0}\right)}{\partial \alpha_{j 1} \partial \alpha_{j 2}}\left(\alpha_{j 1}-\alpha_{j 1}^{0}\right)\left(\alpha_{j 2}-\alpha_{j 2}^{0}\right), \\
R_{k}^{(3)}(\boldsymbol{\alpha})=R_{k}^{(2)}(\boldsymbol{\alpha})+\frac{1}{3 !} \sum_{j 1=1}^{T P} \sum_{j 2=1}^{T P} \sum_{j 3=1}^{T P} \frac{\partial^{3} R_{k}\left(\boldsymbol{\alpha}^{0}\right)}{\partial \alpha_{j 1} \partial \alpha_{j 2} \partial \alpha_{i 3}}\left(\alpha_{j 1}-\alpha_{j 1}^{0}\right)\left(\alpha_{j 2}-\alpha_{j 2}^{0}\right)\left(\alpha_{j 3}-\alpha_{j 3}^{0}\right), \\
R_{k}^{(4)}(\boldsymbol{\alpha})=R_{k}^{(3)}(\boldsymbol{\alpha})+\frac{1}{4 !} \sum_{j 1=1}^{T P} \sum_{j 2=1}^{T P} \sum_{j 3=1}^{T P} \sum_{j 4=1}^{T P} \frac{\partial^{4} R_{k}\left(\boldsymbol{\alpha}^{0}\right)}{\partial \alpha_{j 1} \partial \alpha_{j 2} \partial \alpha_{j 3} \partial \alpha_{j 4}} \\
\times\left[\left(\alpha_{j 1}-\alpha_{j 1}^{0}\right)\left(\alpha_{j 2}-\alpha_{j 2}^{0}\right)\left(\alpha_{j 3}-\alpha_{j 3}^{0}\right)\left(\alpha_{j 4}-\alpha_{j 4}^{0}\right)\right], \\
R_{k}^{(5)}(\boldsymbol{\alpha})=R_{k}^{(4)}(\boldsymbol{\alpha})+\frac{1}{5 !} \sum_{j 1=1}^{T P} \sum_{j 2=1}^{T P} \sum_{j 3=1}^{T P} \sum_{j 4=1}^{T P} \sum_{j 5}^{T P} \frac{\partial^{5} R_{k}\left(\boldsymbol{\alpha}^{0}\right)}{\partial \alpha_{j 1} \partial \alpha_{j 2} \partial \alpha_{j 3} \partial \alpha_{j 4} \partial \alpha_{j 5}} \\
\times\left[\left(\alpha_{j 1}-\alpha_{j 1}^{0}\right)\left(\alpha_{j 2}-\alpha_{j 2}^{0}\right)\left(\alpha_{j 3}-\alpha_{j 3}^{0}\right)\left(\alpha_{j 4}-\alpha_{j 4}^{0}\right)\left(\alpha_{j 5}-\alpha_{j 5}^{0}\right)\right], \\
R_{k}^{(6)}(\boldsymbol{\alpha})=R_{k}^{(5)}(\boldsymbol{\alpha})+\frac{1}{6 !} \sum_{j 1=1}^{T P} \sum_{j 2=1}^{T P} \sum_{j 3=1}^{T P} \sum_{j 4=1}^{T P} \sum_{j 5=1}^{T P} \sum_{j 6=1}^{T P} \frac{\partial^{6} R_{k}\left(\boldsymbol{\alpha}^{0}\right)}{\partial \alpha_{j 1} \partial \alpha_{j 2} \partial \alpha_{j 3} \partial \alpha_{j 4} \partial \alpha_{j 5} \partial \alpha_{j 6}} \\
\times\left[\left(\alpha_{j 1}-\alpha_{j 1}^{0}\right)\left(\alpha_{j 2}-\alpha_{j 2}^{0}\right)\left(\alpha_{j 3}-\alpha_{j 3}^{0}\right)\left(\alpha_{j 4}-\alpha_{j 4}^{0}\right)\left(\alpha_{j 5}-\alpha_{j 5}^{0}\right)\left(\alpha_{j 6}-\alpha_{j 6}^{0}\right)\right] .
\end{gathered}
$$

As is well known, and as indicated by Equations (6)-(12), the Taylor-series of a function of $T P$-variables [e.g., $R_{k}(\boldsymbol{\alpha})$ ] comprises $T P$ 1st-order derivatives, $T P(T P+1) / 2$ distinct 2 nd-order derivatives, $T P(T P+1)(T P+2) / 6$ distinct 3 rd-order derivatives, and so on. The computation by conventional methods of the $n^{\text {th }}$-order functional derivatives (called "sensitivities" in the field of sensitivity analysis) of a response with respect to the $T P$-parameters it depends on would require at least $O\left(T P^{n}\right)$ large-scale computations. This exponential increase - with the order of response sensitivities-of the number of largescale computations needed to determine higher-order sensitivities is the manifestation of the "curse of dimensionality in sensitivity analysis," by analogy to expression coined by Belmann [1] to express the difficulty of using "brute-force" grid search when optimizing a function with many input variables.

Illustrating the Need for High-Order Sensitivities: Neutron Scattering in an Infinite Hydrogenous Medium

The energy-dependent neutron flux, $\Phi(E)$, of scattered of neutrons emitted by an energy-dependent source in a hydrogen-moderated system in which absorption is neglected, is modeled [42-48] by the following integral equation: $Q(E)$

$$
\int_{E}^{\infty} \Phi\left(E^{\prime}\right) \Sigma_{s}\left(E^{\prime}\right) \frac{d E^{\prime}}{E^{\prime}}+Q(E)=\Phi(E) \Sigma_{s}(E) .
$$

The quantity $\Sigma_{S}(E)$ in Equation (13) represents the scattering cross section of hydrogen, having the expression $\Sigma_{s}(E)=N_{H} \sigma_{s}(E)$, where $N_{H}$ denotes the atomic number density of hydrogen while $\sigma_{s}(E)$ denotes the microscopic scattering cross section of hydrogen. The solution of Equation (13) has the following form:

$$
\Phi_{H}(\sigma ; E)=\frac{Q\left(\boldsymbol{\alpha}^{0}\right)}{E N_{H} \sigma_{s}(E)} ; \quad Q(\boldsymbol{\alpha}) \triangleq \int_{E_{s}(\boldsymbol{\alpha})}^{\infty} Q\left(\boldsymbol{\alpha} ; E^{\prime}\right) d E^{\prime}(E)
$$


Although Equation (14) is strictly correct only for an infinite hydrogen medium, it is often used to estimate the energy-dependent flux in water-moderated reactors [18-20]. The effects of the scattering cross sections can be quantified by in isolation from the effects of the other model parameters by considering that the source does not vary from its nominal value denoted as $Q\left(\boldsymbol{\alpha}^{0}\right)$ and that the number density of hydrogen, $N_{H}$, remains unchanged, as well.

Consider that the energy-dependent microscopic total cross section of hydrogen, $\sigma_{S}(E)$, was measured in a series of measurements at an arbitrary but fixed energy value $E_{m}, 0<E_{m}<\infty$, and was found to have a mean value denoted as $\sigma_{m}$. The Taylor-series expansion of $\Phi_{H}(\sigma ; E)$ around $\sigma_{m}$ has the following form:

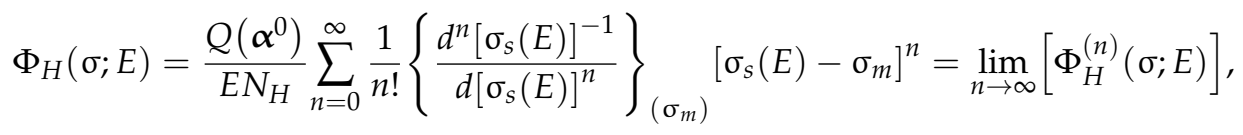

where the quantity $\Phi_{H}^{(n)}(\sigma ; E)$ denotes the " $n^{\text {th }}$-order approximation of the value of the actual flux $\Phi_{H}(\sigma ; E)^{\prime \prime}$, and is defined as follows:

$$
\Phi_{H}^{(n)}(\sigma ; E) \triangleq \frac{Q\left(\boldsymbol{\alpha}^{0}\right)}{E N_{H} \sigma_{m}} \sum_{k=0}^{n}(-1)^{n} t^{n} ; t \triangleq \frac{\sigma_{s}(E)-\sigma_{m}}{\sigma_{m}} .
$$

The Taylor-series in Equation (15) converges only for values of $\sigma_{s}(E)$ confined within the following interval:

$$
(1-\beta) \sigma_{m} \leq \sigma_{s}(E) \leq(1+\beta) \sigma_{m} ; 0<\beta<1 .
$$

It is especially important to ensure that the values of $\sigma_{s}(E)$ used for constructing statistical properties for $\Phi_{H}(\sigma ; E)$ do not fall outside the interval of convergence shown in Equation (17). In particular, it follows from Equation (17) that if a standard deviation, $S D\left[\sigma_{S}(E)\right]$, is also provided (in addition to the measured mean value $\sigma_{m}$ ) for the variate $\sigma_{s}(E)$, then it must be ensured that the Taylor-series shown in Equation (15) is used only for the following range of values:

$$
\beta \sigma_{m} \leq S D\left[\sigma_{s}(E)\right] \leq \beta \sigma_{m} ; 0<\beta<1 .
$$

If a standard deviation is not provided for the measurement of $\sigma_{s}\left(E_{m}\right)$, then the most conservative assumption is to consider that $\sigma_{S}(E)$ is uniformly distributed within the interval provided in Equation (17), in which the Taylor-series of $\Phi_{H}(\sigma ; E)$ convergences, since the uniform distribution is the least informative (hence, the least biased) distribution. It will henceforth be assumed that the distribution for $\sigma_{s}(E)$ is uniform in the interval given in Equation (17). Thus, the uniform probability distribution, $P\left[\sigma_{S}(E)\right]$, the expectation, $\mathbf{E}\left[\sigma_{S}(E)\right]$, the variance, $\operatorname{Var}\left[\sigma_{s}(E)\right]$, and the standard deviation, $S D\left[\sigma_{\mathcal{S}}(E)\right]$, of $\sigma_{s}(E)$ have the following expressions:

$$
\begin{aligned}
& P\left[\sigma_{s}(E)\right]=\frac{1}{2 \beta \sigma_{m}} ; \quad \mathbf{E}\left[\sigma_{s}(E)\right]=\sigma_{m} ; \quad \operatorname{Var}\left[\sigma_{s}(E)\right]=\frac{\left(\beta \sigma_{m}\right)^{2}}{3} ; \\
& S D\left[\sigma_{s}(E)\right]=\frac{1}{\sqrt{3}} \beta \sigma_{m} ; \quad 0<\beta<1 .
\end{aligned}
$$

It follows from the expression given in Equation (15) that the absolute sensitivities of $\Phi_{H}(\sigma ; E)$, which will be denoted as $S_{n}(E), n=1,2, \ldots$, with respect to the parameter $\sigma_{s}(E)$ at energy $E=E_{m}$, where $\sigma_{s}\left(E_{m}\right)=\sigma_{m}$, have the following expressions:

$$
S_{n}(E)=\frac{Q\left(\boldsymbol{\alpha}^{0}\right)}{E N_{H} \sigma_{m}}(-1)^{n}\left[\frac{1}{\sigma_{m}}\right]^{n} .
$$


It follows from the expression in Equation (20) that the relative sensitivities of $\Phi_{H}(\sigma ; E)$ with respect to the parameter $\sigma_{S}(E)$, which will be denoted as $R_{n}(E), n=1,2, \ldots$, have the following expressions:

$$
R_{n}(E)=S_{n}(E) \frac{\left(\sigma_{m}\right)^{n}}{\Phi_{H}\left(\sigma_{m} ; E\right)}=(-1)^{n}, n=1,2, \ldots
$$

It is evident from Equation (21) that the relative values of the sensitivities of all orders are all equal to unity; their values do not decrease with increasing order $n=1,2, \ldots$.

The relative error, $\varepsilon_{\Phi}^{(n)}$, between the exact value of $\Phi_{H}(\sigma ; E)$ provided in Equation (14) and the $n^{\text {th }}$-order approximation $\Phi_{H}^{(n)}(\sigma ; E)$ is defined as follows expression:

$$
\varepsilon_{\Phi}^{(n)} \triangleq \frac{1}{\Phi_{H}(\sigma ; E)}\left[\Phi_{H}(\sigma ; E)-\frac{Q\left(\alpha^{0}\right)}{E N_{H} \sigma_{m}} \sum_{k=0}^{n}(-1)^{k} t^{k}\right]=(-1)^{n+1} t^{n+1} .
$$

It follows from Equation (22) that:

$$
\left|\varepsilon_{\Phi}^{(n)}\right|=\left|t^{n+1}\right|=\left|\left[\frac{\sigma_{s}(E)-\sigma_{m}}{\sigma_{m}}\right]^{n+1}\right|<1 .
$$

As an example of the use of the Taylor-series expansion in sensitivity analysis, consider that it is desired to predict the value of $\Phi_{H}(\sigma ; E)$ when $\sigma_{S}(E)$ is "one standard deviation away from the mean," i.e., when:

$$
\sigma_{s}^{1 S D}(E)=\sigma_{m}+S D\left[\sigma_{s}(E)\right]=\sigma_{m}\left(1+\frac{1}{\sqrt{3}} \beta\right) .
$$

Replacing the result obtained in Equation (24) into the definition provided in Equation (16) and taking $\beta=1-10^{-4}$ (to be extremely close to the outer boundaries of the "one-standard deviation interval") yields the following result when keeping the first four decimal digits:

$$
t=\beta / \sqrt{3}=\left(1-10^{-4}\right) / \sqrt{3} \cong 0.5773
$$

In sensitivity analysis, the value provided in Equation (25) is used in Equation (16) to investigate how accurately the various orders of approximations, $\Phi_{H}^{(n)}(\sigma ; E)$, can predict the actual value of the flux at $\Phi_{H}(\sigma ; E)$. The following results, to four significant decimals, are obtained for $\beta=1-10^{-4}$ and $t=0.5773$ from Equations (16) and (24):

1. The exact value of the flux is:

$$
\Phi_{H}\left(\sigma_{S}^{1 S D} ; E\right)=\frac{Q\left(\boldsymbol{\alpha}^{0}\right)}{E N_{H} \sigma_{m}}\left(1+\frac{1-10^{-4}}{\sqrt{3}}\right)^{-1} \cong 0.6340 \frac{Q\left(\boldsymbol{\alpha}^{0}\right)}{E N_{H} \sigma_{m}} .
$$

2. If only the first-order sensitivities of $\Phi_{H}(\sigma ; E)$ with respect to $\sigma_{S}(E)$ are available, then the 1st-order expansion $(n=1)$ in Equation (16) yields the following result:

$$
\Phi_{H}^{(1)}(\sigma ; E) \triangleq \frac{Q\left(\boldsymbol{\alpha}^{0}\right)}{E N_{H} \sigma_{m}}(1-t)=0.4227 \frac{Q\left(\boldsymbol{\alpha}^{0}\right)}{E N_{H} \sigma_{m}} .
$$

It follows from Equations (26) and (27) that the error of the 1st-order approximation is:

$$
\varepsilon_{\Phi}^{(1)} \triangleq \frac{\Phi_{H}\left(\sigma_{S}^{1 S D} ; E\right)-\Phi_{H}^{(1)}(\sigma ; E)}{\Phi_{H}\left(\sigma_{S}^{1 S D} ; E\right)}=33.33 \% .
$$

Thus, the result obtained by keeping only the first-order sensitivities in the Taylorexpansion underestimates the exact flux by $33.34 \%$. 
3. If first-order and second-order sensitivities of $\Phi_{H}(\sigma ; E)$ with respect to $\sigma_{s}(E)$ are available, then the $2 n d$-order expansion $(n=2)$ in Equation (16) yields the following result:

$$
\Phi_{H}^{(2)}(\sigma ; E) \triangleq \frac{Q\left(\boldsymbol{\alpha}^{0}\right)}{E N_{H} \sigma_{m}}\left(1-t+t^{2}\right)=0.7560 \frac{Q\left(\boldsymbol{\alpha}^{0}\right)}{E N_{H} \sigma_{m}} .
$$

It follows from Equations (29) and (26) that the error of the 2nd-order approximation is as follows:

$$
\varepsilon_{\Phi}^{(2)} \triangleq \frac{\Phi_{H}\left(\sigma_{s}^{1 S D} ; E\right)-\Phi_{H}^{(2)}(\sigma ; E)}{\Phi_{H}\left(\sigma_{s}^{1 S D} ; E\right)}=-19.24 \% .
$$

Thus, the result obtained by keeping the first- and second-order sensitivities in the Taylor-expansion overestimates the exact flux by $19.24 \%$.

4. If all sensitivities up to and including the third-order sensitivities of $\Phi_{H}(\sigma ; E)$ with respect to $\sigma_{s}(E)$ are available, then the 3rd-order expansion $(n=3)$ in Equation (16) yields the following result:

$$
\Phi_{H}^{(3)}(\sigma ; E) \triangleq \frac{Q\left(\boldsymbol{\alpha}^{0}\right)}{E N_{H} \sigma_{m}}\left(1-t+t^{2}-t^{3}\right)=0.5636 \frac{Q\left(\boldsymbol{\alpha}^{0}\right)}{E N_{H} \sigma_{m}} .
$$

It follows from Equations (31) and (26) that the error of the 3rd-order approximation is as follows:

$$
\varepsilon_{\Phi}^{(3)} \triangleq \frac{\Phi_{H}\left(\sigma_{s}^{1 S D} ; E\right)-\Phi_{H}^{(3)}(\sigma ; E)}{\Phi_{H}\left(\sigma_{S}^{1 S D} ; E\right)}=11.11 \%
$$

Thus, the result obtained by keeping the 1st-, 2nd-, and 3rd-order sensitivities in the Taylor-expansion underestimates the exact flux by $11.11 \%$.

Thus, the results obtained by keeping the 1st-, 2nd- and 3rd-order sensitivities in the Taylor-expansion underestimate the exact flux by $11.11 \%$.

5. If all sensitivities up to and including the 4th-order sensitivities of $\Phi_{H}(\sigma ; E)$ with respect to $\sigma_{s}(E)$ are available, then the 4th-order expansion $(n=4)$ in Equation (16) yields the following result:

$$
\Phi_{H}^{(4)}(\sigma ; E) \triangleq \frac{Q\left(\alpha^{0}\right)}{E N_{H} \sigma_{m}}\left(1-t+t^{2}-t^{3}+t^{4}\right)=0.6747 \frac{Q\left(\alpha^{0}\right)}{E N_{H} \sigma_{m}} .
$$

It follows from Equations (33) and (26) that the error of the 4th-order approximation is as follows:

$$
\varepsilon_{\Phi}^{(4)} \triangleq \frac{\Phi_{H}\left(\sigma_{s}^{1 S D} ; E\right)-\Phi_{H}^{(4)}(\sigma ; E)}{\Phi_{H}\left(\sigma_{S}^{1 S D} ; E\right)}=-6.42 \% .
$$

As indicated by the results in Equations (28), (30), (32) and (34), the odd-order approximations underestimate the true value, and the even-order approximations overestimate the true value of the flux, while converging to the actual, true value, of the flux $\Phi_{H}(\sigma ; E)$. This behavior is expected since the flux is represented by an alternating series, cf. Equation (16). The results obtained in Equations (28), (30), (32) and (34) are consistent with the error estimate provided in Equation (22). The relation provided in Equation (23) can be used to determine which order of approximation would need to be used to obtain an approximate result which would be within a predetermined error by comparison to the exact result.

\section{Sixth-Order Moments of the Response Distribution in the Parameter Phase-Space}

In practice, even though the model parameters are not bona fide random quantities, they are considered to be variates distributed according to a multivariate probability distribution function, denoted as $p_{\alpha}(\boldsymbol{\alpha})$. Although $p_{\alpha}(\boldsymbol{\alpha})$ is unknown, it is considered to 
be formally defined on a domain $D_{\alpha}$, so that the various moments of $p_{\alpha}(\boldsymbol{\alpha})$ can be defined in formally as follows:

$$
\langle u(\boldsymbol{\alpha})\rangle_{\alpha} \triangleq \int_{D_{\alpha}} u(\boldsymbol{\alpha}) p_{\alpha}(\boldsymbol{\alpha}) d \boldsymbol{\alpha},
$$

where $u(\alpha)$ is a continuous function of the parameters $\alpha$. Using the general notation introduced in Equation (35), the moments of $p_{\alpha}(\boldsymbol{\alpha})$ are defined as follows:

1. The expectation of a model parameter $\alpha_{j}$, is denoted as $\alpha_{j}^{0}$ and is defined as follows:

$$
\alpha_{j}^{0} \triangleq\left\langle\alpha_{j}\right\rangle_{\alpha} \triangleq \int_{D_{\alpha}} \alpha_{j} p_{\alpha}(\boldsymbol{\alpha}) d \boldsymbol{\alpha}, j=1, \ldots, T P .
$$

The expected values $\alpha_{j}^{0}$ are considered to be the components of the following vector of mean (expected) values:

$$
\alpha^{0} \triangleq\left(\alpha_{1}^{0}, \ldots, \alpha_{T P}^{0}\right)^{\dagger}
$$

2. The covariance, $\operatorname{cov}\left(\alpha_{j 1}, \alpha_{j 2}\right)$, of two parameters, $\alpha_{j 1}$ and $\alpha_{j 2}$, is defined as follows:

$$
\mu_{2}^{j 1 j 2}(\boldsymbol{\alpha}) \triangleq \operatorname{cov}\left(\alpha_{j 1}, \alpha_{j 2}\right) \triangleq\left\langle\left(\alpha_{j 1}-\alpha_{j 1}^{0}\right)\left(\alpha_{j 2}-\alpha_{j 2}^{0}\right)\right\rangle_{\alpha^{\prime}}, j 1, j 2=1, \ldots, T P .
$$

The variance, $\operatorname{var}\left(\alpha_{i}\right)$, of a parameter $\alpha_{i}$, is defined as follows:

$$
\operatorname{var}\left(\alpha_{j}\right) \triangleq\left\langle\left(\alpha_{j}-\alpha_{j}^{0}\right)^{2}\right\rangle_{\alpha}, j=1, \ldots, T P .
$$

The standard deviation, $\sigma_{i}$, of $\alpha_{i}$, is defined as follows: $\sigma_{j} \triangleq \sqrt{\operatorname{var}\left(\alpha_{j}\right)}$. The correlation, $\rho_{j 1 j 2}$, between two parameters $\alpha_{j 1}$ and $\alpha_{j 2}$, is defined as follows:

$$
\rho_{j 1 j 2} \triangleq \operatorname{cov}\left(\alpha_{j 1}, \alpha_{j 2}\right) /\left(\sigma_{j 1} \sigma_{j 2}\right) ; j 1, j 2=1, \ldots, T P
$$

3. The third-order parameter moment, $\mu_{3}^{j 1 j 2 j 3}$, and the associated third-order correlation $t_{j 1 j 2 j 3}$ among three parameters, are defined as follows:

$$
\mu_{3}^{j 1 j 2 j 3} \triangleq\left\langle\left(\alpha_{j 1}-\alpha_{j 1}^{0}\right)\left(\alpha_{j 2}-\alpha_{j 2}^{0}\right)\left(\alpha_{j 3}-\alpha_{j 3}^{0}\right)\right\rangle_{\alpha} \triangleq t_{j 1 j 2 j 3} \sigma_{j 1} \sigma_{j 2} \sigma_{j 3} ; j 1, j 2, j 3=1, \ldots, T P .
$$

4. The fourth-order parameter moment, $\mu_{4}^{j 1 j 2 j 3 j 4}$, is defined as follows:

$$
\begin{aligned}
& \mu_{4}^{i j k l} \triangleq\left\langle\left(\alpha_{j 1}-\alpha_{j 1}^{0}\right)\left(\alpha_{j 2}-\alpha_{j 2}^{0}\right)\left(\alpha_{j 3}-\alpha_{j 3}^{0}\right)\left(\alpha_{j 4}-\alpha_{j 4}^{0}\right)\right\rangle_{\alpha} \\
& \triangleq q_{j 1 j 2 j 3 j 4} \sigma_{j 1} \sigma_{j 2} \sigma_{j 3} \sigma_{j 4} ; j 1, j 2, j 3, j 4=1, \ldots, T P,
\end{aligned}
$$

where $q_{j 1 j 2 j 3 j 4}$ denotes the fourth-order correlation among four parameters.

5. The fifth-order parameter moment, $\mu_{5}^{j 1 j 2 j 3 j 4 j 5}$, is defined as follows:

$$
\begin{aligned}
& \mu_{5}^{j 1 j 2 j 3 j 4 j 5} \triangleq\left\langle\left(\alpha_{j 1}-\alpha_{j 1}^{0}\right)\left(\alpha_{j 2}-\alpha_{j 2}^{0}\right)\left(\alpha_{j 3}-\alpha_{j 3}^{0}\right)\left(\alpha_{j 4}-\alpha_{j 4}^{0}\right)\left(\alpha_{j 5}-\alpha_{j 5}^{0}\right)\right\rangle_{\alpha} \\
& \triangleq p_{j 1 j 2 j 3 j 4 j 5} \sigma_{j 1} \sigma_{j 2} \sigma_{j 3} \sigma_{j 4} \sigma_{j 5} ; j 1, j 2, j 3, j 4, j 5=1, \ldots, T P,
\end{aligned}
$$

where $p_{i j k l m}$ denotes the fifth-order correlation among five parameters.

6. The sixth-order parameter moment, $\mu_{6}^{j 1 j 2 j 3 j 4 j 5 j 6}$, is defined as follows:

$$
\begin{aligned}
& \mu_{6}^{j 1 j 2 j 3 j 4 j 5 j 6} \triangleq\left\langle\left(\alpha_{j 1}-\alpha_{j 1}^{0}\right)\left(\alpha_{j 2}-\alpha_{j 2}^{0}\right) \times \ldots \times\left(\alpha_{j 5}-\alpha_{j 5}^{0}\right)\left(\alpha_{j 6}-\alpha_{j 6}^{0}\right)\right\rangle_{\alpha} \\
& \triangleq s_{j 1 j 2 j 3 j 4 j 5 j 6} \sigma_{j 1} \sigma_{j 2} \sigma_{j 3} \sigma_{j 4} \sigma_{j 5} \sigma_{j 6}, j 1, j 2, j 3, j 4, j 5, j 6=1, \ldots, T P,
\end{aligned}
$$


where $s_{j 1 j 2 j 3 j 4 j 5 j 6}$ denotes the sixth-order correlation among six parameters.

Higher-order moments of the parameter distribution are defined similarly to the first six moments defined in Equations (36)-(44). Uncertainties in the model's parameters will evidently give rise to uncertainties in the model responses $R_{k}(\boldsymbol{\alpha})$. The approximate moments of the unknown distribution of $R_{k}(\boldsymbol{\alpha})$ are obtained by using the so-called "propagation of errors" methodology, integrating formally, over the parameter distribution, various expressions involving the truncated Taylor-series expansion of the response provided in Equation (12). Using this concept of integrating the truncated Taylor-series expansion of the response, Tukey [49] has presented the most extensive formulas to date for the mean, variance, skewness and kurtosis of the response distribution, which include terms up to 4th-order response sensitivities. The remainder of this Section presents formulas which are consistent up to and including the 6th-order standard deviations, thus generalizing the formulas presented by Tukey [49].

\subsection{Expectation Value of a Response}

The expectation (value), $E\left(R_{k}\right)$, of a response $R_{k}(\boldsymbol{\alpha})$ is defined using Equation (35), as follows:

$$
E\left(R_{k}\right) \triangleq\left\langle R_{k}(\boldsymbol{\alpha})\right\rangle_{\alpha} \triangleq \int_{D_{\alpha}} R_{k}(\boldsymbol{\alpha}) p_{\alpha}(\boldsymbol{\alpha}) d \boldsymbol{\alpha} .
$$

Using Equations (6)-(12) in the definition provided in Equation (45) yields the following expressions for the $n^{\text {th }}$-order approximate expectation, $E^{(n)}\left(R_{k}\right), n=1, \ldots, 6$, of the response $R_{k}(\boldsymbol{\alpha})$ :

$$
\begin{gathered}
E^{(1)}\left(R_{k}\right)=E^{(0)}\left(R_{k}\right) \triangleq R_{k}\left(\boldsymbol{\alpha}^{0}\right), \\
E^{(2)}\left(R_{k}\right)=E^{(1)}\left(R_{k}\right)+\frac{1}{2} \sum_{j 1=1}^{T P} \sum_{j 2=1}^{T P} \frac{\partial^{2} R_{k}\left(\boldsymbol{\alpha}^{0}\right)}{\partial \alpha_{j 1} \partial \alpha_{j 2}} \rho_{j 1 j 2} \sigma_{j 1} \sigma_{j 2}, \\
E^{(3)}\left(R_{k}\right)=E^{(2)}\left(R_{k}\right)+\frac{1}{6} \sum_{j 1=1}^{T P} \sum_{j 2=1}^{T P} \sum_{j 3=1}^{T P} \frac{\partial^{3} R_{k}\left(\boldsymbol{\alpha}^{0}\right)}{\partial \alpha_{j 1} \partial \alpha_{j 2} \partial \alpha_{i 3}} t_{j 1 j 2 j 3} \sigma_{j 1} \sigma_{j 2} \sigma_{j 3}, \\
E^{(4)}\left(R_{k}\right)=E^{(3)}\left(R_{k}\right)+\frac{1}{4 !} \sum_{j 1=1}^{T P} \sum_{j 2=1}^{T P} \sum_{j 3=1}^{T P} \sum_{j 4=1}^{T P} \frac{\partial^{4} R_{k}\left(\boldsymbol{\alpha}^{0}\right)}{\partial \alpha_{j 1} \partial \alpha_{j 2} \partial \alpha_{j 3} \partial \alpha_{j 4}} q_{j 1 j 2 j 3 j 4} \sigma_{j 1} \sigma_{j 2} \sigma_{j 3} \sigma_{j 4}, \\
E^{(5)}\left(R_{k}\right)=E^{(4)}\left(R_{k}\right)+\frac{1}{5 !} \sum_{j 1=1}^{T P} \sum_{j 2=1}^{T P} \sum_{j 3=1}^{T P} \sum_{j 4=1}^{T P} \sum_{j 5=1}^{T P} \frac{\partial^{5} R_{k}\left(\boldsymbol{\alpha}^{0}\right)}{\partial \alpha_{j 1} \partial \alpha_{j 2} \partial \alpha_{j 3} \partial \alpha_{j 4} \partial \alpha_{j 5}} \\
\times p_{j 1 j 2 j 3 j 4 j 5} \sigma_{j 1} \sigma_{j 2} \sigma_{j 3} \sigma_{j 4} \sigma_{j 5}, \\
E^{(6)}\left(R_{k}\right)=E^{(5)}\left(R_{k}\right)+\frac{1}{6 !} \sum_{j 1=1}^{T P} \sum_{j 2=1}^{T P} \sum_{j 3=1}^{T P} \sum_{j 4=1}^{T P} \sum_{j 5=1}^{T P} \sum_{j 6=1}^{T P} \frac{\partial^{6} R_{k}\left(\boldsymbol{\alpha}^{0}\right)}{\partial \alpha_{j 1} \partial \alpha_{j 2} \partial \alpha_{j 3} \partial \alpha_{j 4} \partial \alpha_{j 5} \partial \alpha_{j 6}} \\
\times s_{j 1 j 2 j 3 j 4 j 5 j 6} \sigma_{j 1} \sigma_{j 2} \sigma_{j 3} \sigma_{j 4} \sigma_{j 5} \sigma_{j 6} .
\end{gathered}
$$

The radius/domain of convergence of the series in Equation (51) must also be established before using it for any subsequent purpose, since the radius/domain of convergence of the series in Equation (51) may differ from the radius/domain of convergence of the Taylor-series in Equation (12). As indicated in Equation (47), the second-order sensitivities cause the response's expected value to differ from the response's computed value, i.e., $E^{(n)}\left(R_{k}\right) \neq R_{k}\left(\boldsymbol{\alpha}^{0}\right), n \geq 2$.

\subsection{Response Parameter Covariances}

For $n=1, \ldots, 6$, the $n^{\text {th }}$-order approximation of the covariance, $\operatorname{cov}\left(\alpha_{i}, R_{k}\right) \triangleq\left\langle\left(\alpha_{i}-\alpha_{i}^{0}\right)\right.$ $\left.\left[R_{k}(\boldsymbol{\alpha})-E\left(R_{k}\right)\right]\right\rangle_{\alpha}$, between a computed responses and a parameter is defined as follows:

$$
\operatorname{cov}\left(\alpha_{i}, R_{k}^{(n)}\right) \triangleq\left\langle\left(\alpha_{i}-\alpha_{i}^{0}\right)\left[R_{k}^{(n)}(\boldsymbol{\alpha})-E^{(n)}\left(R_{k}\right)\right]\right\rangle_{\alpha}, n=1, \ldots, 6 ; i=1, \ldots, T P .
$$


Using Equations (6)-(12) in Equation (52) yields the following expressions for $i=$ $1, \ldots, T P$ :

$$
\begin{array}{r}
\operatorname{cov}\left(\alpha_{i}, R_{k}^{(1)}\right) \triangleq\left\langle\left(\alpha_{i}-\alpha_{i}^{0}\right) R_{k}^{(1)}(\boldsymbol{\alpha})\right\rangle_{\alpha}=\sum_{j 1=1}^{T P} \frac{\partial R_{k}\left(\boldsymbol{\alpha}^{0}\right)}{\partial \alpha_{j 1}} \rho_{i, j 1} \sigma_{i} \sigma_{j 1} ; \\
\operatorname{cov}\left(\alpha_{i}, R_{k}^{(2)}\right) \triangleq\left\langle\left(\alpha_{i}-\alpha_{i}^{0}\right) R_{k}^{(2)}(\boldsymbol{\alpha})\right\rangle_{\alpha}=\operatorname{cov}\left(\alpha_{i}, R_{k}^{(1)}\right)+\frac{1}{2} \sum_{j 1=1}^{T P} \sum_{j 2=1}^{T P} \frac{\partial^{2} R_{k}\left(\boldsymbol{\alpha}^{0}\right)}{\partial \alpha_{j 1} \partial \alpha_{j 2}} t_{i, j 1 j 2} \sigma_{i} \sigma_{j 1} \sigma_{j 2} ; \\
\operatorname{cov}\left(\alpha_{i}, R_{k}^{(3)}\right) \triangleq\left\langle\left(\alpha_{i}-\alpha_{i}^{0}\right) R_{k}^{(3)}(\boldsymbol{\alpha})\right\rangle_{\alpha}=\operatorname{cov}\left(\alpha_{i}, R_{k}^{(2)}\right) \\
+\frac{1}{6} \sum_{j 1=1}^{T P} \sum_{j 2=1}^{T P} \sum_{j 3=1}^{T P} \frac{\partial^{3} R_{k}\left(\boldsymbol{\alpha}^{0}\right)}{\partial \alpha_{j 1} \partial \alpha_{j 2} \partial \alpha_{j 3}} q_{i, j 1 j 2 j 3} \sigma_{i} \sigma_{j 1} \sigma_{j 2} \sigma_{j 3} ; \\
\operatorname{cov}\left(\alpha_{i}, R_{k}^{(4)}\right) \triangleq\left\langle\left(\alpha_{i}-\alpha_{i}^{0}\right) R_{k}^{(4)}(\boldsymbol{\alpha})\right\rangle_{\alpha}=\operatorname{cov}\left(\alpha_{i}, R_{k}^{(3)}\right) \\
+\frac{1}{4 !} \sum_{j 1=1}^{T P} \sum_{j 2=1}^{T P} \sum_{j 3=1}^{T P} \sum_{j 4=1}^{T P} \frac{\partial^{4} R_{k}\left(\boldsymbol{\alpha}^{0}\right)}{\partial \alpha_{j 1} \partial \alpha_{j 2} \partial \alpha_{j 3} \partial \alpha_{j 4}} p_{i, j 1 j 2 j 3 j 4} \sigma_{i} \sigma_{j 1} \sigma_{j 2} \sigma_{j 3} \sigma_{j 4} ; \\
\operatorname{cov}\left(\alpha_{i}, R_{k}^{(5)}\right) \triangleq\left\langle\left(\alpha_{i}-\alpha_{i}^{0}\right) R_{k}^{(5)}(\boldsymbol{\alpha})\right\rangle_{\alpha}=\operatorname{cov}\left(\alpha_{i}, R_{k}^{(4)}\right) \\
+\frac{1}{5 !} \sum_{j 1=1}^{T P} \sum_{j 2=1}^{T P} \sum_{j=1}^{T P} \sum_{j 4=1}^{T P} \sum_{j 5=1}^{T P} \frac{\partial^{5} R_{k}\left(\boldsymbol{\alpha}^{0}\right)}{\partial \alpha_{j 1} \partial \alpha_{j 2} \partial \alpha_{j 3} \partial \alpha_{j 4} \partial \alpha_{j 5}} s_{i, j 1 j 2 j 3 j 4 j 5} \sigma_{i} \sigma_{j 1} \sigma_{j 2} \sigma_{j 3} \sigma_{j 4} \sigma_{j 5} .
\end{array}
$$

\subsection{Covariance of Two Response}

For $n=1, \ldots, 6$, the $n^{\text {th }}$-order approximation, $\operatorname{cov}\left(R_{k}^{(n)}, R_{\ell}^{(n)}\right)$, of the covariance, $\operatorname{cov}\left(R_{k}, R_{\ell}\right) \triangleq\left\langle\left[R_{k}(\boldsymbol{\alpha})-E\left(R_{k}\right)\right]\left[R_{\ell}(\boldsymbol{\alpha})-E\left(R_{\ell}\right)\right]\right\rangle_{\alpha}$, between two responses $R_{k}$ and $R_{\ell}$ is defined as follows:

$$
\left\{\operatorname{cov}\left(R_{k}^{(n)}, R_{\ell}^{(n)}\right)\right\}_{i n c} \triangleq\left\langle\left[R_{k}^{(n)}(\boldsymbol{\alpha})-E^{(n)}\left(R_{k}\right)\right]\left[R_{\ell}^{(n)}(\boldsymbol{\alpha})-E^{(n)}\left(R_{\ell}\right)\right]\right\rangle_{\alpha} .
$$

It will be seen that the result, denoted as $\left\{\operatorname{cov}\left(R_{k}^{(n)}, R_{\ell}^{(n)}\right)\right\}_{\text {inc }}$, which is provided by the right-side of, is inconsistent (i.e., incomplete) in the approximation of the highest-order term, which contains the product of standard deviations $\sigma_{j 1} \times \sigma_{j 2} \times \ldots \times \sigma_{j n}$. The alternative definition below provides an approximation for a "consistent" covariance, which is denoted as $\left\{\operatorname{cov}\left(R_{k}^{(n)}, R_{\ell}^{(n)}\right)\right\}_{\text {con }}$ and which comprises the consistent (i.e., complete) expression for the term containing $\sigma_{j 1} \times \sigma_{j 2} \times \ldots \times \sigma_{j n}$, when the terms of $O\left(\sigma^{n+1}\right)$ are discarded, i.e.,

$$
\left\{\operatorname{cov}\left(R_{k}^{(n)}, R_{\ell}^{(n)}\right)\right\}_{\operatorname{con}} \triangleq \operatorname{cov}\left(R_{k}^{(n+1)}, R_{\ell}^{(n+1)}\right)-O\left(\sigma^{n+1}\right), n=1, \ldots, 5 .
$$

The standard deviation, $S D\left[R_{k}^{(n)}(\boldsymbol{\alpha})\right]$, of $R_{k}^{(n)}(\boldsymbol{\alpha})$ is obtained by setting $k=\ell$ in either Equation (58) or Equation (59) and taking the square root of the resulting expression, respectively, to obtain:

$$
\begin{gathered}
\left\{S D\left(R_{k}^{(n)}\right)\right\}_{i n c} \triangleq \sqrt{\left\{\operatorname{var}\left(R_{k}^{(n)}, R_{k}^{(n)}\right)\right\}_{i n c}}+O\left(\sigma^{n / 2}\right), n=1,2, \ldots \\
\left\{S D\left(R_{k}^{(n)}\right)\right\}_{\text {con }} \triangleq \sqrt{\left\{\operatorname{var}\left(R_{k}^{(n)}, R_{k}^{(n)}\right)\right\}_{c o n}}, n=1,2, \ldots
\end{gathered}
$$

As indicated by the right-side of Equation (60), the standard deviation $\left\{S D\left(R_{k}^{(n)}\right)\right\}_{\text {inc }}$ corresponding to the inconsistent variance $\left\{\operatorname{var}\left(R_{k}^{(n)}, R_{k}^{(n)}\right)\right\}_{\text {inc }}$ will not comprise the complete number of terms that contain the $n^{\text {th }}$-order parameter standard deviations. In 
contradistinction to $\left\{S D\left(R_{k}^{(n)}\right)\right\}_{i n c}$, the consistent standard deviation, $\left\{S D\left(R_{k}^{(n)}\right)\right\}_{\text {con }}{ }^{\prime}$ which corresponds to the consistent variance $\left\{\operatorname{var}\left(R_{k}^{(n)}, R_{k}^{(n)}\right)\right\}_{\text {inc }}$, will comprise all of the terms that contain the $n^{\text {th }}$-order parameter standard deviations.

The expressions for the covariances and standard deviations of various orders of approximation $n=1, \ldots, 5$, are provided below:

1. The 1st-order approximation of the covariance $\operatorname{cov}\left(R_{k}^{(n)}, R_{\ell}^{(n)}\right)$

$$
\begin{aligned}
& \operatorname{cov}\left(R_{k}^{(1)}, R_{\ell}^{(1)}\right) \triangleq\left\langle\left[R_{k}^{(1)}(\boldsymbol{\alpha})-E^{(1)}\left(R_{k}\right)\right]\left[R_{\ell}^{(1)}(\boldsymbol{\alpha})-E^{(1)}\left(R_{\ell}\right)\right]\right\rangle_{\alpha} \\
& =\sum_{j 1=1}^{N_{\alpha}} \sum_{j 2=1}^{N_{\alpha}} \frac{\partial R_{k}\left(\boldsymbol{\alpha}^{0}\right)}{\partial \alpha_{j 1}} \frac{\partial R_{\ell}\left(\boldsymbol{\alpha}^{0}\right)}{\partial \alpha_{j 2}} \rho_{j 1 j 2} \sigma_{j 1} \sigma_{j 2},
\end{aligned}
$$

Notably, the expression of $\operatorname{cov}\left(R_{k}^{(1)}, R_{\ell}^{(1)}\right)$ provided in Equation (62) is consistent both in the highest-order (in this case: first-order) of sensitivities and also in the highest-order of parameter standard deviation (in this case, second-order) since all of the terms involving the product $\sigma_{j 1} \sigma_{j 2}$ are consistently included (i.e., none are missing) in the expression provided. Therefore, the standard deviation will also be correct to first-order in the standard deviations of the parameters, i.e.,

$$
S D\left(R_{k}^{(1)}\right)=\left\{\sum_{j 1=1}^{T P} \sum_{j 2=1}^{T P} \frac{\partial R_{k}\left(\boldsymbol{\alpha}^{0}\right)}{\partial \alpha_{j 1}} \frac{\partial R_{\ell}\left(\boldsymbol{\alpha}^{0}\right)}{\partial \alpha_{j 2}} \rho_{j 1 j 2} \sigma_{j 1} \sigma_{j 2}\right\}^{\frac{1}{2}}
$$

2. The 2 nd-order approximation of the covariance $\operatorname{cov}\left(R_{k}^{(n)}, R_{\ell}^{(n)}\right)$ :

(i) Using Equation (58) yields the following expression:

$$
\begin{aligned}
& \left\{\operatorname{cov}\left(R_{k}^{(2)}, R_{\ell}^{(2)}\right)\right\}_{i n c}=\operatorname{cov}\left(R_{k}^{(1)}, R_{\ell}^{(1)}\right) \\
& +\frac{1}{2} \sum_{j 1=1}^{T P} \sum_{j 2=1}^{T P} \sum_{j 3=1}^{T P}\left(\frac{\partial^{2} R_{k}\left(\boldsymbol{\alpha}^{0}\right)}{\partial \alpha_{j 1} \partial \alpha_{j 2}} \frac{\partial R_{\ell}\left(\boldsymbol{\alpha}^{0}\right)}{\partial \alpha_{j 3}}+\frac{\partial R_{k}\left(\boldsymbol{\alpha}^{0}\right)}{\partial \alpha_{j 1}} \frac{\partial^{2} R_{\ell}\left(\boldsymbol{\alpha}^{0}\right)}{\partial \alpha_{j 2} \partial \alpha_{j 3}}\right) t_{j 1 j 2 j 3} \sigma_{j 1} \sigma_{j 2} \sigma_{j 3} \\
& +\frac{1}{4} \sum_{j 1=1}^{T P} \sum_{j 2=1}^{T P} \sum_{j 3=1}^{T P} \sum_{j 4=1}^{T P} \frac{\partial^{2} R_{k}\left(\boldsymbol{\alpha}^{0}\right)}{\partial \alpha_{j 1} \partial \alpha_{j 2}} \frac{\partial^{2} R_{\ell}\left(\boldsymbol{\alpha}^{0}\right)}{\partial \alpha_{j 3} \partial \alpha_{j 4}}\left(q_{j 1 j 2 j 34}-\rho_{j 1 j 2} \rho_{j 3 j 4}\right) \sigma_{j 1} \sigma_{j 2} \sigma_{j 3} \sigma_{j 4} .
\end{aligned}
$$

(ii) Using Equation (59) yields the following expression:

$$
\begin{aligned}
& \left\{\operatorname{cov}\left(R_{k}^{(2)}, R_{\ell}^{(2)}\right)\right\}_{\operatorname{con}}=\left\{\operatorname{cov}\left(R_{k}^{(2)}, R_{\ell}^{(2)}\right)\right\}_{i n c} \\
& +\frac{1}{6} \sum_{j 1=1}^{T P} \sum_{j 2=1}^{T P} \sum_{j 3=1}^{T P} \sum_{j 4=1}^{T P}\left(\frac{\partial^{3} R_{k}\left(\boldsymbol{\alpha}^{0}\right)}{\partial \alpha_{j 1} \partial \alpha_{j 2} \partial \alpha_{j 3}} \frac{\partial R_{\ell}\left(\boldsymbol{\alpha}^{0}\right)}{\partial \alpha_{j 4}}+\frac{\partial R_{k}\left(\boldsymbol{\alpha}^{0}\right)}{\partial \alpha_{j 1}} \frac{\partial^{3} R_{\ell}\left(\boldsymbol{\alpha}^{0}\right)}{\partial \alpha_{j 2} \partial \alpha_{j 3} \partial \alpha_{j 4}}\right) q_{j 1 j 2 j 34} \sigma_{j 1} \sigma_{j 2} \sigma_{j 3} \sigma_{j 4} .
\end{aligned}
$$

3. Notably, the expression of $\left\{\operatorname{cov}\left(R_{k}^{(2)}, R_{\ell}^{(2)}\right)\right\}_{\text {inc }}$ provided in Equation (64) is consistent in the 2nd-order of sensitivities but is inconsistent in the 4th-order of standard deviations of parameters, i.e., $O\left(\sigma_{j 1} \sigma_{j 2} \sigma_{j 3} \sigma_{j 4}\right)$. On the other hand, the expression $\left\{\operatorname{cov}\left(R_{k}^{(2)}, R_{\ell}^{(2)}\right)\right\}_{\text {con }}$ provided in Equation (65) is consistent in the 4th-order (i.e., $\left.\sigma_{j 1} \sigma_{j 2} \sigma_{j 3} \sigma_{j 4}\right)$ of parameter standard deviations. Therefore, if the 3rd-order sensitivities are available, then the expression provided in Equation (65) should be used, since it is correct up to, and including, the fourth-order terms containing the products $\sigma_{j 1} \sigma_{j 2} \sigma_{j 3} \sigma_{j 4}$. Consequently, the consistent second-order standard deviation $\left\{S D\left(R_{k}^{(2)}\right)\right\}_{\text {con }} \triangleq \sqrt{\left\{\operatorname{cov}\left(R_{k}^{(2)}, R_{\ell}^{(2)}\right)\right\}_{\text {con }}}$ will not have any 2nd-order errors in the parameter standard deviations. In contradistinction, the 2nd-order inconsistent standard deviation $\left\{S D\left(R_{k}^{(2)}\right)\right\}_{\text {inc }} \triangleq \sqrt{\left\{\operatorname{cov}\left(R_{k}^{(2)}, R_{\ell}^{(2)}\right)\right\}_{i n c}}$ will have second-order errors in the parameter standard deviations. 
4. The 3rd-order approximation of the covariance $\operatorname{cov}\left(R_{k}^{(3)}, R_{\ell}^{(3)}\right)$ :

(i) Using Equation (58) yields the following expression:

$$
\begin{aligned}
& \left\{\operatorname{cov}\left(R_{k}^{(3)}, R_{\ell}^{(3)}\right)\right\}_{i n c}=\frac{1}{12} \sum_{j 1=1}^{T P} \sum_{j 2=1}^{T P} \sum_{j 3=1}^{T P} \sum_{j 4=1}^{T P} \sum_{j 5=1}^{T P}\left[\frac{\partial^{3} R_{k}\left(\boldsymbol{\alpha}^{0}\right)}{\partial \alpha_{j 1} \partial \alpha_{j 2} \partial \alpha_{j 3}} \frac{\partial^{2} R_{\ell}\left(\boldsymbol{\alpha}^{0}\right)}{\partial \alpha_{j 4} \partial \alpha_{j 5}}\left(p_{j 1 j 2 j 3 j 4 j 5}-t_{j 1 j 2 j 3} \rho_{j 4 j 5}\right)\right. \\
& \left.+\frac{\partial^{2} R_{k}\left(\boldsymbol{\alpha}^{0}\right)}{\partial \alpha_{j 1} \partial \alpha_{j 2}} \frac{\partial^{3} R_{\ell}\left(\boldsymbol{\alpha}^{0}\right)}{\partial \alpha_{j 3} \partial \alpha_{j 4} \partial \alpha_{j 5}}\left(p_{j 1 j 2 j 3 j 4 j 5}-\rho_{j 1 j 2} t_{j 3 j 4 j 5}\right)\right] \sigma_{j 1} \sigma_{j 2} \sigma_{j 3} \sigma_{j 4} \sigma_{j 5}+\left\{\operatorname{cov}\left(R_{k}^{(2)}, R_{\ell}^{(2)}\right)\right\}_{c o n} .
\end{aligned}
$$

(ii) Using Equation (59) yields the following expression:

$$
\begin{aligned}
\left\{\operatorname{cov}\left(R_{k}^{(3)}, R_{\ell}^{(3)}\right)\right\}_{c o n} & =\left\{\operatorname{cov}\left(R_{k}^{(3)}, R_{\ell}^{(3)}\right)\right\}_{i n c}+\frac{1}{24} \sum_{j 1=1}^{T P} \sum_{j 2=1}^{T P} \sum_{j 3=1}^{T P} \sum_{j 4=1}^{T P} \sum_{j 5=1}^{T P}\left[\frac{\partial^{4} R_{k}\left(\boldsymbol{\alpha}^{0}\right)}{\partial \alpha_{j 1} \partial \alpha_{j 2} \partial \alpha_{j 3} \partial \alpha_{j 4}} \frac{\partial R_{\ell}\left(\boldsymbol{\alpha}^{0}\right)}{\partial \alpha_{j 5}}\right. \\
& \left.+\frac{\partial R_{k}\left(\boldsymbol{\alpha}^{0}\right)}{\partial \alpha_{j 1}} \frac{\partial^{4} R_{\ell}\left(\boldsymbol{\alpha}^{0}\right)}{\partial \alpha_{j 2} \partial \alpha_{j 3} \partial \alpha_{j 4} \partial \alpha_{j 5}}\right] p_{j 1 j 2 j 3 j 4 j 5} \sigma_{j 1} \sigma_{j 2} \sigma_{j 3} \sigma_{j 4} \sigma_{j 5} .
\end{aligned}
$$

The expression of $\left\{\operatorname{cov}\left(R_{k}^{(3)}, R_{\ell}^{(3)}\right)\right\}_{\text {inc }}$ provided in Equation (66) is consistent in the third-order sensitivities but is inconsistent in the highest-order of parameter standard deviations, i.e., $O\left(\sigma_{j 1} \sigma_{j 2} \sigma_{j 3} \sigma_{j 4} \sigma_{j 5}\right)$, while the expression $\left\{\operatorname{cov}\left(R_{k}^{(3)}, R_{\ell}^{(3)}\right)\right\}_{\text {con }}$ provided in Equation (67) is consistent in the highest-order of parameter standard deviations. Therefore, if the 4th-order sensitivities are available, then the expression provided in Equation (67) should be used, since it is correct up to, and including, the fifth-order terms containing the products $\sigma_{j 1} \sigma_{j 2} \sigma_{j 3} \sigma_{j 4} \sigma_{j 5}$. Also noteworthy is the fact that neither the consistent nor the inconsistent standard deviations computed using the 3rd-order variance approximations provided in Equation (66) or Equation(67), respectively, are correct to third-order (i.e., $\sqrt{\sigma_{j 1} \sigma_{j 2} \sigma_{j 3} \sigma_{j 4} \sigma_{j 5} \sigma_{j 6}}$ ) parameter standard deviations. In order to obtain standard deviations which are correct up to and includ-

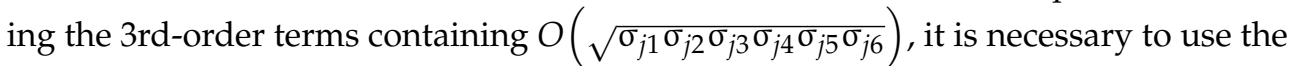
4th-order variance approximations to be provided below.

5. The 4th-order approximation of the covariance $\operatorname{cov}\left(R_{k}^{(n)}, R_{\ell}^{(n)}\right)$ :

(i) Using Equation (58) yields the following expression:

$$
\begin{aligned}
& \left\{\operatorname{cov}\left(R_{k}^{(4)}, R_{\ell}^{(4)}\right)\right\}_{i n c}=\left\{\operatorname{cov}\left(R_{k}^{(3)}, R_{\ell}^{(3)}\right)\right\}_{c o n} \\
& +\frac{1}{36} \sum_{j 1=1}^{J_{\alpha}} \sum_{j 2=1}^{J_{\alpha}} \sum_{j 3=1}^{J_{\alpha}} \sum_{j 4=1}^{N_{\alpha}} \sum_{j 5=1}^{N_{\alpha}} \sum_{j \sigma=1}^{N_{\alpha}} \frac{\partial^{3} R_{k}\left(\boldsymbol{\alpha}^{0}\right)}{\partial \alpha_{j 1} \partial \alpha_{j 2} \partial \alpha_{j 3}} \frac{\partial^{3} R_{l}\left(\boldsymbol{\alpha}^{0}\right)}{\partial \alpha_{j 4} \partial \alpha_{j 5} \partial \alpha_{j 6}} \\
& \times\left(s_{j 1 j 2 j 3 j 4 j 5 j 6}-t_{j 1 j 2 j 3} t_{j 4 j 5 j 6}\right) \sigma_{j 1} \sigma_{j 2} \sigma_{j 3} \sigma_{j 4} \sigma_{j 5} \sigma_{j 6} \\
& +\frac{1}{48} \sum_{j 1=1}^{N_{\alpha}} \sum_{j 2=1}^{N_{\alpha}} \sum_{j 3=1}^{N_{\alpha}} \sum_{j 4=1}^{N_{\alpha}} \sum_{j 5=1}^{N_{\alpha}} \sum_{j \sigma=1}^{N_{\alpha}} \frac{\partial^{4} R_{k}\left(\boldsymbol{\alpha}^{0}\right)}{\partial \alpha_{j 1} \partial \alpha_{j 2} \partial \alpha_{j 3} \partial \alpha_{j 4}} \frac{\partial^{2} R_{l}\left(\boldsymbol{\alpha}^{0}\right)}{\partial \alpha_{j 5} \partial \alpha_{j 6}} \\
& \times\left(s_{j 1 j 2 j 3 j 4 j 5 j 6}-q_{j 1 j 2 j 3 j 4} \rho_{j 5 j 6}\right) \sigma_{j 1} \sigma_{j 2} \sigma_{j 3} \sigma_{j 4} \sigma_{j 5} \sigma_{j 6} \\
& +\frac{1}{48} \sum_{j 1=1}^{J_{\alpha}} \sum_{j 2=1}^{J_{\alpha}} \sum_{j 3=1}^{N_{\alpha}} \sum_{j 4=1}^{N_{\alpha}} \sum_{j 5=1}^{N_{\alpha}} \sum_{j \sigma=1}^{N_{\alpha}} \frac{\partial^{2} R_{k}\left(\boldsymbol{\alpha}^{0}\right)}{\partial \alpha_{j 1} \partial \alpha_{j 2}} \frac{\partial^{4} R_{l}\left(\boldsymbol{\alpha}^{0}\right)}{\partial \alpha_{j 3} \partial \alpha_{j 4} \partial \alpha_{j 5} \partial \alpha_{j 6}} \\
& \times\left(s_{j 1 j 2 j 3 j 4 j 5 j 6}-\rho_{j 1 j 2} q_{j 3 j 4 j 5 j 6}\right) \sigma_{j 1} \sigma_{j 2} \sigma_{j 3} \sigma_{j 4} \sigma_{j 5} \sigma_{j 6} .
\end{aligned}
$$

(ii) Using Equation (59) yields the following expression:

$$
\begin{aligned}
& \left\{\operatorname{cov}\left(R_{k}^{(4)}, R_{\ell}^{(4)}\right)\right\}_{c o n}=\left\{\operatorname{cov}\left(R_{k}^{(4)}, R_{\ell}^{(4)}\right)\right\}_{i n c}+\frac{1}{120} \sum_{j 1=1}^{T P} \sum_{j 2=1}^{T P} \sum_{j 3=1}^{T P} \sum_{j 4=1}^{T P} \sum_{j 5=1}^{T P} \sum_{j 6=1}^{T P}\left[\frac{\partial^{5} R_{k}\left(\boldsymbol{\alpha}^{0}\right)}{\partial \alpha_{j 1} \partial \alpha_{j 2} \partial \alpha_{j 3} \partial \alpha_{j 4} \partial \alpha_{j 5}}\right. \\
& \left.\times \frac{\partial R_{l}\left(\boldsymbol{\alpha}^{0}\right)}{\partial \alpha_{j 6}}+\frac{\partial R_{k}\left(\boldsymbol{\alpha}^{0}\right)}{\partial \alpha_{j 1}} \frac{\partial^{5} R_{l}\left(\boldsymbol{\alpha}^{0}\right)}{\partial \alpha_{j 2} \partial \alpha_{j 3} \partial \alpha_{j 4} \partial \alpha_{j 5} \partial \alpha_{j 6}}\right] s_{j 1 j 2 j 3 j 4 j 5 j 6} \sigma_{j 1} \sigma_{j 2} \sigma_{j 3} \sigma_{j 4} \sigma_{j 5} \sigma_{j 6} .
\end{aligned}
$$


The expression of $\left\{\operatorname{cov}\left(R_{k}^{(4)}, R_{\ell}^{(4)}\right)\right\}_{\text {inc }}$ provided in Equation (68) is consistent in the 4th-order of sensitivities but is inconsistent in the highest-order (6th-order) of parameter standard deviations, while the expression $\left\{\operatorname{cov}\left(R_{k}^{(3)}, R_{\ell}^{(3)}\right)\right\}_{\operatorname{con}}$ provided in Equation (67) is consistent in the 6th-order of parameter standard deviations $\sigma_{j 1} \sigma_{j 2} \sigma_{j 3} \sigma_{j 4} \sigma_{j 5} \sigma_{j 6}$ Therefore, if the 5th-order sensitivities are available, then the expression provided in Equation (67) should be used, since it is correct up to, and including, the fifth-order terms containing the products $\sigma_{j 1} \sigma_{j 2} \sigma_{j 3} \sigma_{j 4} \sigma_{j 5} \sigma_{j 6}$. The standard deviation $\left\{S D\left(R_{k}^{(4)}\right)\right\}_{\text {con }} \triangleq$ $\sqrt{\left\{\operatorname{cov}\left(R_{k}^{(4)}, R_{\ell}^{(4)}\right)\right\}_{\operatorname{con}}}$ would comprise all of the 3rd-order standard deviations of the form $\sqrt{\sigma_{j 1} \sigma_{j 2} \sigma_{j 3} \sigma_{j 4} \sigma_{j 5} \sigma_{j 6}}$.

\subsection{Triple Correlations among Responses and Parameters}

A. The triple-correlations (or third-order moment of the distribution of responses), denoted as $\mu_{3}\left(R_{k}, R_{\ell}, R_{m}\right)$, among three responses $R_{k}(\boldsymbol{\alpha}), R_{\ell}(\boldsymbol{\alpha})$ and $R_{m}(\boldsymbol{\alpha})$, are defined as follows:

$$
\mu_{3}\left(R_{k}, R_{\ell}, R_{m}\right) \triangleq\left\langle\left[R_{k}(\boldsymbol{\alpha})-E\left(R_{k}\right)\right]\left[R_{\ell}(\boldsymbol{\alpha})-E\left(R_{\ell}\right)\right]\left[R_{m}(\boldsymbol{\alpha})-E\left(R_{m}\right)\right]\right\rangle_{\alpha} .
$$

1. If only first-order response sensitivities are available, the following 1st-order approximate expression, denoted as $\mu_{3}^{(1)}\left(R_{k}, R_{\ell}, R_{m}\right)$, can be obtained:

$$
\mu_{3}^{(1)}\left(R_{k}, R_{\ell}, R_{m}\right)=\sum_{j 1=1}^{T P} \sum_{j 2=1}^{T P} \sum_{j 3=1}^{T P} \frac{\partial R_{k}\left(\boldsymbol{\alpha}^{0}\right)}{\partial \alpha_{j 1}} \frac{\partial R_{\ell}\left(\boldsymbol{\alpha}^{0}\right)}{\partial \alpha_{j 2}} \frac{\partial R_{m}\left(\boldsymbol{\alpha}^{0}\right)}{\partial \alpha_{j 3}} t_{j 1 j 2 j 3} \sigma_{j 1} \sigma_{j 2} \sigma_{j 3} .
$$

2. If 1 st- and 2nd-order response sensitivities are available, the following 2 nd-order approximate expression, denoted as $\mu_{3}^{(2)}\left(R_{k}, R_{\ell}, R_{m}\right)$, can be obtained:

$$
\begin{aligned}
& \mu_{3}^{(2)}\left(R_{k}, R_{\ell}, R_{m}\right)=\mu_{3}^{(1)}\left(R_{k}, R_{\ell}, R_{m}\right)+\mu_{3}^{(2)}\left(\sigma^{5}\right)+\mu_{3}^{(2)}\left(\sigma^{6}\right) \\
& +\frac{1}{2} \sum_{j 1=1}^{N_{\alpha}} \sum_{j 2=1}^{N_{\alpha}} \sum_{j=1}^{N_{\alpha}} \sum_{j 4=1}^{N_{\alpha}}\left[\frac{\partial R_{k}\left(\boldsymbol{\alpha}^{0}\right)}{\partial \alpha_{j 1}} \frac{\partial R_{\ell}\left(\boldsymbol{\alpha}^{0}\right)}{\partial \alpha_{j 2}} \frac{\partial^{2} R_{m}\left(\boldsymbol{\alpha}^{0}\right)}{\partial \alpha_{j 3} \partial \alpha_{j 4}}\left(q_{j 1 j 2 j 3 j 4}-\rho_{j 1 j 2} \rho_{j 3 j 4}\right)\right. \\
& \left.+\frac{\partial R_{k}\left(\boldsymbol{\alpha}^{0}\right)}{\partial \alpha_{j 1}} \frac{\partial^{2} R_{\ell}\left(\boldsymbol{\alpha}^{0}\right)}{\partial \alpha_{j 2} \partial \alpha_{j 3}} \frac{\partial R_{m}\left(\boldsymbol{\alpha}^{0}\right)}{\partial \alpha_{j 4}}\left(q_{j 1 j 2 j 3 j 4}-\rho_{j 1 j 4} \rho_{j 2 j 3}\right)\right] \sigma_{j 1} \sigma_{j 2} \sigma_{j 3} \sigma_{j 4},
\end{aligned}
$$

where the quantity $\mu_{3}^{(2)}\left(\sigma^{5}\right)$ contains products of the form $\sigma_{j 1} \sigma_{j 2} \sigma_{j 3} \sigma_{j 4} \sigma_{j 5}$ and is defined as follows:

$$
\begin{aligned}
& \mu_{3}^{(2)}\left(\sigma^{5}\right) \triangleq \frac{1}{4} \sum_{j 1=1}^{T P} \sum_{j 2=1}^{T P} \sum_{j 3=1}^{T P} \sum_{j 4=1}^{T P} \sum_{j 5=1}^{T P}\left[\frac{\partial R_{k}\left(\boldsymbol{\alpha}^{0}\right)}{\partial \alpha_{j 1}} \frac{\partial^{2} R_{\ell}\left(\boldsymbol{\alpha}^{0}\right)}{\partial \alpha_{j 2} \partial \alpha_{j 3}} \frac{\partial^{2} R_{m}\left(\boldsymbol{\alpha}^{0}\right)}{\partial \alpha_{j 4} \partial \alpha_{j 5}}\right. \\
& \times\left(p_{j 1 j 2 j 3 j 4 j 5}-t_{j 122 j 3} \rho_{j 4 j 5}-t_{j 1 j 4 j 5} \rho_{j 2 j 3}\right)+\frac{\partial^{2} R_{k}\left(\boldsymbol{\alpha}^{0}\right)}{\partial \alpha_{j 1} \partial \alpha_{j 2}} \frac{\partial R_{\ell}\left(\boldsymbol{\alpha}^{0}\right)}{\partial \alpha_{j 3}} \frac{\partial^{2} R_{m}\left(\boldsymbol{\alpha}^{0}\right)}{\partial \alpha_{j 4} \partial \alpha_{j 5}} \\
& \times\left(p_{j 1 j 2 j 3 j 4 j 5}-\rho_{j 1 j 2} t_{j 3 j 4 j 5}-t_{j 1 j 2 j 3} \rho_{j 4 j 5}\right)+\frac{\partial^{2} R_{k}\left(\boldsymbol{\alpha}^{0}\right)}{\partial \alpha_{j 1} \partial \alpha_{j 2}} \frac{\partial^{2} R_{\ell}\left(\boldsymbol{\alpha}^{0}\right)}{\partial \alpha_{j 3} \partial \alpha_{j 4}} \frac{\partial R_{m}\left(\boldsymbol{\alpha}^{0}\right)}{\partial \alpha_{j 5}} \\
& \left.\times\left(p_{j 1 j 2 j 34 j 55}-\rho_{j 1 j 2} t_{j 3 j 4 j 5}-t_{j 1 j 2 j 5} \rho_{j 3 j 4}\right)\right] \sigma_{j 1} \sigma_{j 2} \sigma_{j 3} \sigma_{j 4} \sigma_{j 5},
\end{aligned}
$$

while the quantity $\mu_{3}^{(2)}\left(\sigma^{6}\right)$ contains products of the form $\sigma_{j 1} \sigma_{j 2} \sigma_{j 3} \sigma_{j 4} \sigma_{j 5} \sigma_{j 6}$ and is defined as follows:

$$
\begin{aligned}
& \mu_{3}^{(2)}\left(\sigma^{6}\right) \triangleq \frac{1}{8} \sum_{j 1=1}^{T P} \sum_{j 2=1}^{T P} \sum_{j 3=1}^{T P} \sum_{j 4=1}^{T P} \sum_{j 5=1}^{T P} \sum_{j 6=1}^{T P} \frac{\partial^{2} R_{k}\left(\boldsymbol{\alpha}^{0}\right)}{\partial \alpha_{j 1} \partial \alpha_{j 2}} \frac{\partial^{2} R_{\ell}\left(\boldsymbol{\alpha}^{0}\right)}{\partial \alpha_{j 3} \partial \alpha_{j 4}} \frac{\partial^{2} R_{m}\left(\boldsymbol{\alpha}^{0}\right)}{\partial \alpha_{j 5} \partial \alpha_{j 6}} \sigma_{j 1} \sigma_{j 2} \sigma_{j 3} \sigma_{j 4} \sigma_{j 5} \sigma_{j 6} \\
& \left(s_{j 1 j 2 j 3 j 4 j 5 j 6}+2 \rho_{j 1 j 2} \rho_{j 3 j 4} \rho_{j 5 j 6}-\rho_{j 1 j 2} q_{j 3 j 4 j 5 j 6}-q_{j 1 j 2 j 5 j 6} \rho_{j 3 j 4}-q_{j 1 j 2 j 3 j 4} \rho_{j 5 j 6}\right) .
\end{aligned}
$$

As indicated in Equation (74), the expression of $\mu_{3}^{(2)}\left(R_{k}, R_{\ell}, R_{m}\right)$ consistently contains all of the 1st- and 2nd-order response sensitivities, and all of the 
5th-order terms in standard deviations (of the form $\sigma_{j 1} \sigma_{j 2} \sigma_{j 3} \sigma_{j 4} \sigma_{j 5}$ ) but does not contain all of the 6th-order terms in standard deviations (of the form $\left.\sigma_{j 1} \sigma_{j 2} \sigma_{j 3} \sigma_{j 4} \sigma_{j 5} \sigma_{j 6}\right)$ The 6th-order terms in standard deviations, containing the remaining terms of the form $\sigma_{j 1} \sigma_{j 2} \sigma_{j 3} \sigma_{j 4} \sigma_{j 5} \sigma_{j 6}$ are provided below.

3. The availability of the third-order response sensitivities enables the computation of additional terms that comprise 6th-order terms containing the products $\sigma_{j 1} \sigma_{j 2} \sigma_{j 3} \sigma_{j 4} \sigma_{j 5} \sigma_{j 6}$. The following 3rd-order (in sensitivities) approximate expression, denoted as $\mu_{3}^{(3)}\left(R_{k}, R_{\ell}, R_{m}\right)$, can be obtained:

$$
\begin{aligned}
& \mu_{3}^{(3)}\left(R_{k}, R_{l}, R_{m}\right) \triangleq \frac{1}{12} \sum_{j 1=1}^{T P} \sum_{j 2=1}^{T P} \sum_{j 3=1}^{T P} \sum_{j 4=1}^{T P} \sum_{j 5=1}^{T P} \sum_{j 6=1}^{T P}\left[\frac{\partial R_{k}\left(\boldsymbol{\alpha}^{0}\right)}{\partial \alpha_{j 1}} \frac{\partial^{2} R_{l}\left(\boldsymbol{\alpha}^{0}\right)}{\partial \alpha_{j 2} \partial \alpha_{j 3}} \frac{\partial^{3} R_{m}\left(\boldsymbol{\alpha}^{0}\right)}{\partial \alpha_{j 4} \partial \alpha_{j 5} \partial \alpha_{j 6}}\right. \\
& \times\left(s_{j 1 j 2 j 3 j 4 j 5 j 6}-t_{j 1 j 2 j 3} t_{j 4 j 5 j 6}-q_{j 1 j 4 j 5 j 6} \rho_{j 2 j 3}\right)+\frac{\partial R_{k}\left(\boldsymbol{\alpha}^{0}\right)}{\partial \alpha_{i_{1}}} \frac{\partial^{3} R_{l}\left(\boldsymbol{\alpha}^{0}\right)}{\partial \alpha_{i_{2}} \partial \alpha_{i_{3}} \partial \alpha_{i_{4}}} \frac{\partial^{2} R_{m}\left(\boldsymbol{\alpha}^{0}\right)}{\partial \alpha_{i 5} \partial \alpha_{i}} \\
& \times\left(s_{j 1 j 2 j 3 j 4 j 5 j 6}-t_{j 1 j 5 j 6} t_{j 2 j 3 j 4}-q_{j 1 j 2 j 3 j 4} \rho_{j 5 j 6}\right)+\frac{\partial^{2} R_{k}\left(\boldsymbol{\alpha}^{0}\right)}{\partial \alpha_{j 1} \partial \alpha_{j 2}} \frac{\partial R_{l}\left(\boldsymbol{\alpha}^{0}\right)}{\partial \alpha_{j 3}} \frac{\partial^{3} R_{m}\left(\boldsymbol{\alpha}^{0}\right)}{\partial \alpha_{j 4} \partial \alpha_{j 5} \partial \alpha_{j 6}} \\
& \times\left(s_{j 1 j 2 j 3 j 4 j 5 j 6}-t_{j 1 j 2 j 3} t_{j 4 j 5 j 6}-\rho_{j 1 j 2} q_{j 3 j 4 j 5 j 6}\right)+\frac{\partial^{2} R_{k}\left(\boldsymbol{\alpha}^{0}\right)}{\partial \alpha_{j 1} \partial \alpha_{j 2}} \frac{\partial^{3} R_{l}\left(\boldsymbol{\alpha}^{0}\right)}{\partial \alpha_{j 3} \partial \alpha_{j 4} \partial \alpha_{j 5}} \frac{\partial R_{m}\left(\boldsymbol{\alpha}^{0}\right)}{\partial \alpha_{j 6}} \\
& \times\left(s_{j 1 j 2 j 3 j 4 j 5 j 6}-t_{j 1 j 2 j 6} t_{j 3 j 4 j 5}-\rho_{j 1 j 2} q_{j 3 j 4 j 5 j 6}\right)+\frac{\partial^{3} R_{l}\left(\boldsymbol{\alpha}^{0}\right)}{\partial \alpha_{j 1} \partial \alpha_{j 2} \partial \alpha_{j 3}} \frac{\partial R_{l}\left(\boldsymbol{\alpha}^{0}\right)}{\partial \alpha_{j 4}} \frac{\partial^{2} R_{m}\left(\boldsymbol{\alpha}^{0}\right)}{\partial \alpha_{j 5} \partial \alpha_{j 6}} \\
& \times\left(s_{j 1 j 2 j 3 j 4 j 5 j 6}-t_{j 1 j 2 j 3} t_{j 4 j 5 j 6}-q_{j 1 j 2 j 3 j 4} \rho_{j 5 j 6}\right)+\frac{\partial^{3} R_{l}\left(\boldsymbol{\alpha}^{0}\right)}{\partial \alpha_{j 1} \partial \alpha_{j 2} \partial \alpha_{j 3}} \frac{\partial^{2} R_{l}\left(\boldsymbol{\alpha}^{0}\right)}{\partial \alpha_{j 4} \partial \alpha_{j 5}} \frac{\partial R_{m}\left(\boldsymbol{\alpha}^{0}\right)}{\partial \alpha_{j 6}} \\
& \left.\times\left(s_{j 1 j 2 j 3 j 4 j 5 j 6}-t_{j 1 j 2 j 3} t_{j 4 j 5 j 6}-q_{j 1 j 2 j 3 j 6} \rho_{j 4 j 5}\right)\right] \sigma_{j 1} \sigma_{j 2} \sigma_{j 3} \sigma_{j 4} \sigma_{j 5} \sigma_{j 6}+\mu_{3}^{(2)}\left(R_{k}, R_{\ell}, R_{m}\right) .
\end{aligned}
$$

4. If fourth-order response sensitivities are also available, the following 4th-order (in sensitivities) approximate expression $\mu_{3}^{(4)}\left(R_{k}, R_{l}, R_{m}\right)$ can be obtained:

$$
\begin{aligned}
& \mu_{3}^{(4)}\left(R_{k}, R_{l}, R_{m}\right) \triangleq \frac{1}{24} \sum_{j 1=1}^{T P} \sum_{j 2=1}^{T P} \sum_{j 3=1}^{T P} \sum_{j 4=1}^{T P} \sum_{j 5=1}^{T P} \sum_{j 6=1}^{T P}\left[\frac{\partial R_{k}\left(\boldsymbol{\alpha}^{0}\right)}{\partial \alpha_{j 1}} \frac{\partial R_{l}\left(\boldsymbol{\alpha}^{0}\right)}{\partial \alpha_{j 2}} \frac{\partial^{4} R_{m}\left(\boldsymbol{\alpha}^{0}\right)}{\partial \alpha_{j 3} \partial \alpha_{j 4} \partial \alpha_{j 5} \partial \alpha_{j 6}}\right. \\
& \times\left(s_{j 1 j 2 j 3 j 4 j 5 j 6}-\rho_{j 1 j 2} q_{j 3 j 4 j 5 j 6}\right)+\frac{\partial R_{k}\left(\boldsymbol{\alpha}^{0}\right)}{\partial \alpha_{j 1}} \frac{\partial^{4} R_{l}\left(\boldsymbol{\alpha}^{0}\right)}{\partial \alpha_{j 2} \partial \alpha_{j 3} \partial \alpha_{j 4} \partial \alpha_{j 5}} \frac{\partial R_{m}\left(\boldsymbol{\alpha}^{0}\right)}{\partial \alpha_{j 6}} \\
& \times\left(s_{j 1 j 2 j 3 j 4 j 5 j 6}-\rho_{j 1 j 6} q_{j 2 j 3 j 4 j 5}\right)+\frac{\partial^{4} R_{k}\left(\boldsymbol{\alpha}^{0}\right)}{\partial \alpha_{j 1} \partial \alpha_{j 2} \partial \alpha_{j 3} \partial \alpha_{j 4}} \frac{\partial R_{l}\left(\boldsymbol{\alpha}^{0}\right)}{\partial \alpha_{j 5}} \frac{\partial R_{m}\left(\boldsymbol{\alpha}^{0}\right)}{\partial \alpha_{j 6}} \\
& \left.\times\left(s_{j 1 j 2 j 3 j 4 j 5 j 6}-q_{j 1 j 2 j 3 j 4} \rho_{j 5 j 6}\right)\right] \sigma_{j 1} \sigma_{j 2} \sigma_{j 3} \sigma_{j 4} \sigma_{j 5} \sigma_{j 6}+\mu_{3}^{(3)}\left(R_{k}, R_{l}, R_{m}\right) .
\end{aligned}
$$

As can be observed from Equation (76), the quantity $\mu_{3}^{(4)}\left(R_{k}, R_{l}, R_{m}\right)$ contains all of the terms involving 6 th-order products $\sigma_{j 1} \sigma_{j 2} \sigma_{j 3} \sigma_{j 4} \sigma_{j 5} \sigma_{j 6}$ of standard deviations.

B. The triple-correlations among one parameter, $\alpha_{i}$, and two responses, $R_{k}(\alpha)$ and $R_{\ell}(\boldsymbol{\alpha})$, are defined as follows:

$$
\mu_{3}\left(\alpha_{i}, R_{k}, R_{\ell}\right) \triangleq\left\langle\left(\alpha_{i}-\alpha_{i}^{0}\right)\left[R_{k}(\boldsymbol{\alpha})-E\left(R_{k}\right)\right]\left[R_{\ell}(\boldsymbol{\alpha})-E\left(R_{\ell}\right)\right]\right\rangle_{\alpha} .
$$

Using the Taylor series up to and including the 6th-order in standard deviations, the expression of $\mu_{3}\left(\alpha_{i}, R_{k}, R_{\ell}\right)$ is as follows:

$$
\begin{aligned}
& \mu_{3}\left(\alpha_{i}, R_{k}, R_{\ell}\right)=\sum_{j 1=1}^{T P} \sum_{j 2=1}^{T P} \frac{\partial R_{j}\left(\boldsymbol{\alpha}^{0}\right)}{\partial \alpha_{j 1}} \frac{\partial R_{k}\left(\boldsymbol{\alpha}^{0}\right)}{\partial \alpha_{j 2}} t_{j 1 j 2 i} \sigma_{j 1} \sigma_{j 2} \sigma_{i}+\frac{1}{2} \sum_{j 1=1}^{T P} \sum_{j 2=1}^{T P} \sum_{j 3=1}^{T P}\left[\frac{\partial R_{j}\left(\boldsymbol{\alpha}^{0}\right)}{\partial \alpha_{j 1}} \frac{\partial^{2} R_{k}\left(\boldsymbol{\alpha}^{0}\right)}{\partial \alpha_{j 2} \partial \alpha_{j 3}}\right. \\
& \left.\times\left(q_{j 1 j 2 j 3 i}-\rho_{j 1 i} \rho_{j 2 j 3}\right)+\frac{\partial^{2} R_{j}\left(\boldsymbol{\alpha}^{0}\right)}{\partial \alpha_{j 1} \partial \alpha_{j 2}} \frac{\partial R_{k}\left(\boldsymbol{\alpha}^{0}\right)}{\partial \alpha_{j 3}}\left(q_{j 1 j 2 j 3 i}-\rho_{j 1 j 2} \rho_{j 3 i}\right)\right] \sigma_{j 1} \sigma_{j 2} \sigma_{j 3} \sigma_{i} \\
& +\mu_{5}^{(5)}\left(\alpha_{i}, R_{k}, R_{\ell}\right)+\mu_{5}^{(6)}\left(\alpha_{i}, R_{k}, R_{\ell}\right),
\end{aligned}
$$

where the quantity $\mu_{5}^{(5)}\left(\alpha_{i}, R_{k}, R_{\ell}\right)$ comprises terms in 5th-order standard deviations involving products of the form $\sigma_{j 1} \sigma_{j 2} \sigma_{j 3} \sigma_{j 4} \sigma_{i}$, and is defined as follows: 


$$
\begin{aligned}
& \mu_{3}^{(5)}\left(\alpha_{i}, R_{k}, R_{\ell}\right) \triangleq \frac{1}{4} \sum_{j 1=1}^{T P} \sum_{j 2=1}^{T P} \sum_{j 3=1}^{T P} \sum_{j 4=1}^{T P} \frac{\partial^{2} R_{j}\left(\boldsymbol{\alpha}^{0}\right)}{\partial \alpha_{j 1} \partial \alpha_{j 2}} \frac{\partial^{2} R_{k}\left(\boldsymbol{\alpha}^{0}\right)}{\partial \alpha_{j 3} \partial \alpha_{j 4}}\left(p_{j 1 j 2 j 3 j 4 i}-t_{j 1 j 2 i} \rho_{j 3 j 4}-\rho_{j 1 j 2} t_{j 3 j 4 i}\right) \\
& \times \sigma_{j 1} \sigma_{j 2} \sigma_{j 3} \sigma_{j 4} \sigma_{i}+\frac{1}{6} \sum_{j 1=1}^{T P} \sum_{j 2=1}^{T P} \sum_{j 3=1}^{T P} \sum_{j 4=1}^{T P}\left[\frac{\partial R_{j}\left(\boldsymbol{\alpha}^{0}\right)}{\partial \alpha_{j 1}} \frac{\partial^{3} R_{k}\left(\boldsymbol{\alpha}^{0}\right)}{\partial \alpha_{j 2} \partial \alpha_{j 3} \partial \alpha_{j 4}}\left(p_{j 1 j 2 j 3 j 4 i}-\rho_{j 1 i} t_{j 2 j 3 j 4}\right)\right. \\
& \left.+\frac{\partial^{3} R_{j}\left(\boldsymbol{\alpha}^{0}\right)}{\partial \alpha_{j 1} \partial \alpha_{j 2} \partial \alpha_{j 3}} \frac{\partial R_{k}\left(\boldsymbol{\alpha}^{0}\right)}{\partial \alpha_{j 4}} \times\left(p_{j 1 j 2 j 3 j 4 i}-t_{j 1 j 2 j 3} \rho_{j 4 i}\right)\right] \sigma_{j 1} \sigma_{j 2} \sigma_{j 3} \sigma_{j 4} \sigma_{i},
\end{aligned}
$$

while the quantity $\mu_{5}^{(6)}\left(\alpha_{i}, R_{k}, R_{\ell}\right)$ comprises terms in 6th-order standard deviations involving products of the form $\sigma_{j 1} \sigma_{j 2} \sigma_{j 3} \sigma_{j 4} \sigma_{j 5} \sigma_{i}$, and is defined as follows:

$$
\begin{aligned}
& \mu_{5}^{(6)}\left(\alpha_{i}, R_{k}, R_{\ell}\right) \triangleq \frac{1}{12} \sum_{j 1=1}^{T P} \sum_{j 2=1}^{T P} \sum_{j 3=1}^{T P} \sum_{j 4=1}^{T P} \sum_{j 5=1}^{T P}\left[\frac{\partial^{2} R_{j}\left(\boldsymbol{\alpha}^{0}\right)}{\partial \alpha_{j 1} \partial \alpha_{j 2}} \frac{\partial^{3} R_{k}\left(\boldsymbol{\alpha}^{0}\right)}{\partial \alpha_{j 3} \partial \alpha_{j 4} \partial \alpha_{j 5}}\right. \\
& \times\left(s_{j 1 j 2 j 3 j 4 j 5 i}-t_{j 1 j 2 i} t_{j 3 j 4 j 5}-\rho_{j 1 j 2} q_{j 3 j 4 j 5 i}\right)+\frac{\partial^{3} R_{j}\left(\boldsymbol{\alpha}^{0}\right)}{\partial \alpha_{j 1} \partial \alpha_{j 2} \partial \alpha_{j 3}} \frac{\partial^{2} R_{k}\left(\boldsymbol{\alpha}^{0}\right)}{\partial \alpha_{j 4} \partial \alpha_{j 5}} \\
& \left.\times\left(s_{j 1 j 2 j 3 j 4 j 5 i}-t_{j 1 j 2 j 3} t_{j 4 j 5 i}-q_{j 1 j 2 j 3 i} \rho_{j 4 j 5}\right)\right] \sigma_{j 1} \sigma_{j 2} \sigma_{j 3} \sigma_{j 4} \sigma_{j 5} \sigma_{i} \\
& +\frac{1}{24} \sum_{j 1=1}^{T P} \sum_{j 2=1}^{T P} \sum_{j 3=1}^{T P} \sum_{j 4=1}^{T P} \sum_{j 5=1}^{T P}\left[\frac{\partial R_{j}\left(\boldsymbol{\alpha}^{0}\right)}{\partial \alpha_{j 1}} \frac{\partial^{4} R_{k}\left(\boldsymbol{\alpha}^{0}\right)}{\partial \alpha_{j 2} \partial \alpha_{j 3} \partial \alpha_{j 4} \partial \alpha_{j 5}}\left(s_{j 1 j 2 j 3 j 4 j 5 i}-\rho_{j 1 i} q_{j 2 j 3 j 4 j 5}\right)\right. \\
& \left.+\frac{\partial^{4} R_{j}\left(\boldsymbol{\alpha}^{0}\right)}{\partial \alpha_{j 1} \partial \alpha_{j 2} \partial \alpha_{j 3} \partial \alpha_{j 4}} \frac{\partial R_{k}\left(\boldsymbol{\alpha}^{0}\right)}{\partial \alpha_{j 5}}\left(s_{j 1 j 2 j 3 j 4 j 5 i}-q_{j 1 j 2 j 3 j 4} \rho_{j 5 i}\right)\right] \sigma_{j 1} \sigma_{j 2} \sigma_{j 3} \sigma_{j 4} \sigma_{j 5} \sigma_{i} .
\end{aligned}
$$

C. The triple-correlations among two parameters $\alpha_{i}, \alpha_{j}$ and one response $R_{k}(\alpha)$ are defined as follows:

$$
\mu_{3}\left(\alpha_{i}, \alpha_{j}, R_{k}\right) \triangleq\left\langle\left(\alpha_{i}-\alpha_{i}^{0}\right)\left(\alpha_{j}-\alpha_{j}^{0}\right)\left[R_{k}(\boldsymbol{\alpha})-E\left(R_{k}\right)\right]\right\rangle_{\alpha}
$$

Using the Taylor series up to and including the 6th-order in standard deviations, the expression of $\mu_{3}\left(\alpha_{i}, \alpha_{j}, R_{k}\right)$ is as follows:

$$
\begin{aligned}
& \mu_{3}\left(\alpha_{i}, \alpha_{j}, R_{k}\right)=\sum_{j 1=1}^{T P} \frac{\partial R_{k}\left(\boldsymbol{\alpha}^{0}\right)}{\partial \alpha_{j 1}} t_{j 1 i j} \sigma_{j 1} \sigma_{i} \sigma_{j}+\frac{1}{2} \sum_{j 1=1}^{T P} \sum_{j 2=1}^{T P} \frac{\partial^{2} R_{k}\left(\boldsymbol{\alpha}^{0}\right)}{\partial \alpha_{j 1} \partial \alpha_{j 2}}\left(q_{j 1 j 2 i j}-\rho_{j 1 j 2} \rho_{i j}\right) \sigma_{j 1} \sigma_{j 2} \sigma_{i} \sigma_{j} \\
& +\frac{1}{6} \sum_{j 1=1}^{T P} \sum_{j 2=1}^{T P} \sum_{j 3=1}^{T P} \frac{\partial^{3} R_{k}\left(\boldsymbol{\alpha}^{0}\right)}{\partial \alpha_{j 1} \partial \alpha_{j 2} \partial \alpha_{j 3}}\left(p_{j 1 j 2 j 3 i j}-t_{j 1 j 2 j 3} \rho_{i j}\right) \sigma_{j 1} \sigma_{j 2} \sigma_{j 3} \sigma_{i} \sigma_{j} \\
& +\frac{1}{24} \sum_{j 1=1}^{T P} \sum_{j 2=1}^{T P} \sum_{j 3=1}^{T P} \sum_{j 4=1}^{T P} \frac{\partial^{4} R_{k}\left(\boldsymbol{\alpha}^{0}\right)}{\partial \alpha_{j 1} \partial \alpha_{j 2} \partial \alpha_{j 3} \partial \alpha_{j 4}}\left(s_{j 1 j 2 j 3 j 4 i j}-q_{j 1 j 2 j 3 j} \rho_{i j}\right) \sigma_{j 1} \sigma_{j 2} \sigma_{j 3} \sigma_{j 4} \sigma_{i} \sigma_{j} .
\end{aligned}
$$

\subsection{Quadruple Correlations among Responses and Parameters}

A. The quadruple-correlations (or fourth-order moment of the distribution of responses), denoted as $\mu_{4}\left(R_{k}, R_{\ell}, R_{m}, R_{n}\right)$, among four responses $R_{k}(\boldsymbol{\alpha}), R_{\ell}(\boldsymbol{\alpha}), R_{m}(\boldsymbol{\alpha})$ and $R_{n}(\boldsymbol{\alpha})$, are defined as follows:

$$
\begin{aligned}
& \mu_{4}\left(R_{k}, R_{\ell}, R_{m}, R_{n}\right) \triangleq\left\langle\left[R_{k}(\boldsymbol{\alpha})-E\left(R_{k}\right)\right]\left[R_{\ell}(\boldsymbol{\alpha})-E\left(R_{\ell}\right)\right]\right. \\
& \left.\times\left[R_{m}(\boldsymbol{\alpha})-E\left(R_{m}\right)\right]\left[R_{n}(\boldsymbol{\alpha})-E\left(R_{n}\right)\right]\right\rangle_{\alpha} .
\end{aligned}
$$

1. If only first-order response sensitivities are available, the following 1st-order approximate expression, denoted as $\mu_{4}^{(1)}\left(R_{k}, R_{l}, R_{m}, R_{n}\right)$, can be obtained:

$$
\begin{gathered}
\mu_{4}^{(1)}\left(R_{k}, R_{l}, R_{m}, R_{n}\right) \triangleq\left\langle\left[R_{k}^{(1)}(\boldsymbol{\alpha})-E^{(1)}\left(R_{k}\right)\right]\left[R_{\ell}^{(1)}(\boldsymbol{\alpha})-E^{(1)}\left(R_{\ell}\right)\right]\right. \\
\left.\times\left[R_{m}^{(1)}(\boldsymbol{\alpha})-E^{(1)}\left(R_{m}\right)\right]\left[R_{n}^{(1)}(\boldsymbol{\alpha})-E^{(1)}\left(R_{n}\right)\right]\right\rangle_{\alpha} \\
=\sum_{j 1=1}^{T P} \sum_{j 2=1}^{T P} \sum_{j 3=1}^{T P} \sum_{j 4=1}^{T P} \frac{\partial R_{k}\left(\boldsymbol{\alpha}^{0}\right)}{\partial \alpha_{j 1}} \frac{\partial R_{l}\left(\boldsymbol{\alpha}^{0}\right)}{\partial \alpha_{j 2}} \frac{\partial R_{m}\left(\boldsymbol{\alpha}^{0}\right)}{\partial \alpha_{j 3}} \frac{\partial R_{n}\left(\boldsymbol{\alpha}^{0}\right)}{\partial \alpha_{j 4}} q_{j 1 j 2 j 34} \sigma_{j 1} \sigma_{j 2} \sigma_{j 3} \sigma_{j 4} .
\end{gathered}
$$


The expression of $\mu_{4}^{(1)}\left(R_{k}, R_{l}, R_{m}, R_{n}\right)$ is consistent in that it comprises all of the 1st-order sensitivities and all of the terms involving 4 th-order products of standard deviations of the form $\sigma_{j 1} \sigma_{j 2} \sigma_{j 3} \sigma_{j 4}$.

2. If all 1st-and 2nd-order response sensitivities are available, the following approximation of the quadruple-correlations among four responses $R_{k}(\alpha), R_{\ell}(\boldsymbol{\alpha})$, $R_{m}(\boldsymbol{\alpha})$ and $R_{n}(\boldsymbol{\alpha})$ can be obtained:

$$
\begin{aligned}
& \mu_{4}^{(2)}\left(R_{k}, R_{\ell}, R_{m}, R_{n}\right) \triangleq \mu_{4}^{(1)}\left(R_{k}, R_{\ell}, R_{m}, R_{n}\right)+\mu_{4}^{(2, a)}\left(\sigma^{6}\right)+\mu_{4}^{(2, b)}\left(\sigma^{6}\right) \\
& +\frac{1}{2} \sum_{j 1=1}^{T P} \sum_{j 2=1}^{T P} \sum_{j 3=1}^{T P} \sum_{j 4=1}^{T P} \sum_{j 5=1}^{T P}\left[\frac{\partial R_{k}\left(\boldsymbol{\alpha}^{0}\right)}{\partial \alpha_{j 1}} \frac{\partial R_{\ell}\left(\boldsymbol{\alpha}^{0}\right)}{\partial \alpha_{j 2}} \frac{\partial R_{m}\left(\boldsymbol{\alpha}^{0}\right)}{\partial \alpha_{j 3}} \frac{\partial^{2} R_{n}\left(\boldsymbol{\alpha}^{0}\right)}{\partial \alpha_{j 4} \partial \alpha_{j 5}}\right. \\
& \times\left(p_{j 1 j 2 j 3 j 4 j 5}-t_{j 1 j 2 j 3} \rho_{j 4 j 5}\right)+\frac{\partial R_{k}\left(\boldsymbol{\alpha}^{0}\right)}{\partial \alpha_{j 1}} \frac{\partial R_{\ell}\left(\boldsymbol{\alpha}^{0}\right)}{\partial \alpha_{j 2}} \frac{\partial^{2} R_{m}\left(\boldsymbol{\alpha}^{0}\right)}{\partial \alpha_{j 3} \partial \alpha_{j 4}} \frac{\partial R_{n}\left(\boldsymbol{\alpha}^{0}\right)}{\partial \alpha_{j 5}}\left(p_{j 1 j 2 j 3 j 4 j 5}-t_{j 1 j 2 j 5} \rho_{j 3 j 4}\right) \\
& +\frac{\partial R_{k}\left(\boldsymbol{\alpha}^{0}\right)}{\partial \alpha_{j 1}} \frac{\partial^{2} R_{\ell}\left(\boldsymbol{\alpha}^{0}\right)}{\partial \alpha_{j 2} \partial \alpha_{j 3}} \frac{\partial R_{m}\left(\boldsymbol{\alpha}^{0}\right)}{\partial \alpha_{j 4}} \frac{\partial R_{n}\left(\boldsymbol{\alpha}^{0}\right)}{\partial \alpha_{j 5}}\left(p_{j 1 j 2 j 3 j 4 j 5}-t_{j 1 j 4 j 5} \rho_{j 2 j 3}\right) \\
& \left.+\frac{\partial^{2} R_{k}\left(\boldsymbol{\alpha}^{0}\right)}{\partial \alpha_{j 1} \partial \alpha_{j 2}} \frac{\partial R_{\ell}\left(\boldsymbol{\alpha}^{0}\right)}{\partial \alpha_{j 3}} \frac{\partial R_{m}\left(\boldsymbol{\alpha}^{0}\right)}{\partial \alpha_{j 4}} \frac{\partial R_{n}\left(\boldsymbol{\alpha}^{0}\right)}{\partial \alpha_{j 5}}\left(p_{j 1 j 2 j 3 j 4 j 5}-\rho_{j 1 j 2} t_{j 3 j 4 j 5}\right)\right] \sigma_{j 1} \sigma_{j 2} \sigma_{j 3} \sigma_{j 4} \sigma_{j 5},
\end{aligned}
$$

where the quantities $\mu_{4}^{(2, a)}\left(\sigma^{6}\right)$ and $\mu_{4}^{(2, b)}\left(\sigma^{6}\right)$ contain the terms involving 1 storder and 2nd-order sensitivities but 6 th-order products of standard deviations of the form $\sigma_{j 1} \sigma_{j 2} \sigma_{j 3} \sigma_{j 4} \sigma_{j 5} \sigma_{j 6}$, and are defined as follows:

$$
\begin{aligned}
& \mu_{4}^{(2, a)}\left(\sigma^{6}\right) \triangleq \frac{1}{4} \sum_{j 1=1}^{T P} \sum_{j 2=1}^{T P} \sum_{j 3=1}^{T P} \sum_{j 4=1}^{T P} \sum_{j 5=1}^{T P} \sum_{j 6=1}^{T P}\left[\frac{\partial R_{k}\left(\boldsymbol{\alpha}^{0}\right)}{\partial \alpha_{j 1}} \frac{\partial R_{\ell}\left(\boldsymbol{\alpha}^{0}\right)}{\partial \alpha_{j 2}} \frac{\partial^{2} R_{m}\left(\boldsymbol{\alpha}^{0}\right)}{\partial \alpha_{j 3} \partial \alpha_{j 4}} \frac{\partial^{2} R_{n}\left(\boldsymbol{\alpha}^{0}\right)}{\partial \alpha_{j 5} \partial \alpha_{j 6}}\right. \\
& \times\left(s_{j 1 j 2 j 3 j 4 j 5 j 6}+\rho_{j 1 j 2} \rho_{j 3 j 4} \rho_{j 5 j 6}-q_{j 1 j 2 j 5 j 6} \rho_{j 3 j 4}-q_{j 1 j 2 j 3 j 4} \rho_{j 5 j 6}\right) \\
& +\frac{\partial R_{k}\left(\boldsymbol{\alpha}^{0}\right)}{\partial \alpha_{j 1}} \frac{\partial^{2} R_{\ell}\left(\boldsymbol{\alpha}^{0}\right)}{\partial \alpha_{j 2} \partial \alpha_{j 3}} \frac{\partial R_{m}\left(\boldsymbol{\alpha}^{0}\right)}{\partial \alpha_{j 4}} \frac{\partial^{2} R_{n}\left(\boldsymbol{\alpha}^{0}\right)}{\partial \alpha_{j 5} \partial \alpha_{j 6}} \\
& \times\left(s_{j 1 j 2 j 3 j 4 j 5 j 6}+\rho_{j 1 j 4} \rho_{j 2 j 3} \rho_{j 5 j 6}-q_{j 1 j 4 j 5 j 6} \rho_{j 2 j 3}-q_{j 1 j 2 j 3 j 4} \rho_{j 5 j 6}\right) \\
& +\frac{\partial R_{k}\left(\boldsymbol{\alpha}^{0}\right)}{\partial \alpha_{j 1}} \frac{\partial^{2} R_{\ell}\left(\boldsymbol{\alpha}^{0}\right)}{\partial \alpha_{j 2} \partial \alpha_{j 3}} \frac{\partial^{2} R_{m}\left(\boldsymbol{\alpha}^{0}\right)}{\partial \alpha_{j 4} \partial \alpha_{j 5}} \frac{\partial R_{n}\left(\boldsymbol{\alpha}^{0}\right)}{\partial \alpha_{j 6}} \\
& \left.\times\left(s_{j 1 j 2 j 3 j 4 j 5 j 6}+\rho_{j 1 j 6} \rho_{j 2 j 3} \rho_{j 4 j 5}-q_{j 1 j 4 j 5 j 6} \rho_{j 2 j 3}-q_{j 1 j 2 j 3 j 6} \rho_{j 4 j 5}\right)\right] \sigma_{j 1} \sigma_{j 2} \sigma_{j 3} \sigma_{j 4} \sigma_{j 5} \sigma_{j 6} \\
& \mu_{4}^{(2, b)}\left(\sigma^{6}\right) \triangleq \frac{1}{4} \sum_{j 1=1}^{T P} \sum_{j 2=1}^{T P} \sum_{j 3=1}^{T P} \sum_{j 4=1}^{T P} \sum_{j 5=1}^{T P} \sum_{j 6=1}^{T P}\left[\frac{\partial^{2} R_{k}\left(\boldsymbol{\alpha}^{0}\right)}{\partial \alpha_{j 1} \partial \alpha_{j 2}} \frac{\partial R_{\ell}\left(\boldsymbol{\alpha}^{0}\right)}{\partial \alpha_{j 3}} \frac{\partial R_{m}\left(\boldsymbol{\alpha}^{0}\right)}{\partial \alpha_{j 4}} \frac{\partial^{2} R_{n}\left(\boldsymbol{\alpha}^{0}\right)}{\partial \alpha_{j 5} \partial \alpha_{j 6}}\right. \\
& \times\left(s_{j 1 j 2 j 3 j 4 j 5 j 6}+\rho_{j 1 j 2} \rho_{j 3 j 4} \rho_{j 5 j 6}-\rho_{j 1 j 2} q_{j 3 j 4 j 5 j 6}-q_{j 1 j 2 j 3 j 4} \rho_{j 5 j 6}\right) \\
& +\frac{\partial^{2} R_{k}\left(\boldsymbol{\alpha}^{0}\right)}{\partial \alpha_{j 1} \partial \alpha_{j 2}} \frac{\partial R_{\ell}\left(\boldsymbol{\alpha}^{0}\right)}{\partial \alpha_{j 3}} \frac{\partial^{2} R_{m}\left(\boldsymbol{\alpha}^{0}\right)}{\partial \alpha_{j 4} \partial \alpha_{j 5}} \frac{\partial R_{n}\left(\boldsymbol{\alpha}^{0}\right)}{\partial \alpha_{j 6}} \\
& \times\left(s_{j 1 j 2 j 3 j 4 j 5 j 6}+\rho_{j 1 j 2} \rho_{j 3 j 6} \rho_{j 4 j 5}-\rho_{j 1 j 2} q_{j 3 j 4 j 5 j 6}-q_{j 1 j 2 j 3 j 6} \rho_{j 4 j 5}\right) \\
& +\frac{\partial^{2} R_{k}\left(\boldsymbol{\alpha}^{0}\right)}{\partial \alpha_{j 1} \partial \alpha_{j 2}} \frac{\partial^{2} R_{l}\left(\boldsymbol{\alpha}^{0}\right)}{\partial \alpha_{j 3} \partial \alpha_{j 4}} \frac{\partial R_{m}\left(\boldsymbol{\alpha}^{0}\right)}{\partial \alpha_{j 5}} \frac{\partial R_{n}\left(\boldsymbol{\alpha}^{0}\right)}{\partial \alpha_{j 6}} \\
& \left.\times\left(s_{j 1 j 2 j 3 j 4 j 5 j 6}+\rho_{j 1 j 2} \rho_{j 3 j 4} \rho_{j 5 j 6}-\rho_{j 1 j 2} q_{j 3 j 4 j 5 j 6}-q_{j 1 j 2 j 5 j 6} \rho_{j 3 j 4}\right)\right] \sigma_{j 1} \sigma_{j 2} \sigma_{j 3} \sigma_{j 4} \sigma_{j 5} \sigma_{j 6}
\end{aligned}
$$

3. If third-order response sensitivities are also available, then the remaining terms containing 6th-order products of standard deviations of the form $\sigma_{j 1} \sigma_{j 2} \sigma_{j 3} \sigma_{j 4} \sigma_{j 5} \sigma_{j 6}$ can also be computed, to obtain the following approximation consistent in 6thorder products of standard deviations, denoted as $\mu_{4}^{(3)}\left(R_{k}, R_{l}, R_{m}, R_{n}\right)$ :

$$
\begin{aligned}
& \mu_{4}^{(3)}\left(R_{k}, R_{\ell}, R_{m}, R_{n}\right) \triangleq \mu_{4}^{(2)}\left(R_{k}, R_{\ell}, R_{m}, R_{n}\right) \\
& +\frac{1}{6} \sum_{j 1=1}^{T P} \sum_{j 2=1}^{T P} \sum_{j 3=1} \sum_{j 4=1} \sum_{j 5=1} \sum_{j 6=1}^{T P}\left[\frac{\partial R_{k}\left(\boldsymbol{\alpha}^{0}\right)}{\partial \alpha_{j 1}} \frac{\partial R_{l}\left(\boldsymbol{\alpha}^{0}\right)}{\partial \alpha_{j 2}} \frac{\partial R_{m}\left(\boldsymbol{\alpha}^{0}\right)}{\partial \alpha_{j 3}} \frac{\partial^{3} R_{n}\left(\boldsymbol{\alpha}^{0}\right)}{\partial \alpha_{j 4} \partial \alpha_{j 5} \partial \alpha_{j 6}}\right. \\
& \times\left(s_{j 1 j 2 j 3 j 4 j 5 j 6}-t_{j 1 j 2 j 3} t_{j 4 j 5 j 6}\right)+\frac{\partial R_{k}\left(\boldsymbol{\alpha}^{0}\right)}{\partial \alpha_{j 1}} \frac{\partial R_{l}\left(\boldsymbol{\alpha}^{0}\right)}{\partial \alpha_{j 2}} \frac{\partial^{3} R_{l}\left(\boldsymbol{\alpha}^{0}\right)}{\partial \alpha_{j 3} \partial \alpha_{j 4} \partial \alpha_{j 5}} \frac{\partial R_{n}\left(\boldsymbol{\alpha}^{0}\right)}{\partial \alpha_{j 6}} \\
& \times\left(s_{j 1 j 2 j 3 j 4 j 5 j 6}-t_{j 1 j 2 j 6} t_{j 3 j 4 j 5}\right)+\frac{\partial R_{k}\left(\boldsymbol{\alpha}^{0}\right)}{\partial \alpha_{j 1}} \frac{\partial^{3} R_{l}\left(\boldsymbol{\alpha}^{0}\right)}{\partial \alpha_{j 2} \partial \alpha_{j 3} \partial \alpha_{j 4}} \frac{\partial R_{m}\left(\boldsymbol{\alpha}^{0}\right)}{\partial \alpha_{j 5}} \frac{\partial R_{n}\left(\boldsymbol{\alpha}^{0}\right)}{\partial \alpha_{j 6}} \\
& \times\left(s_{j 1 j 2 j 3 j 4 j 5 j 6}-t_{j 1 j 5 j 6} t_{j 2 j 3 j 4}\right)+\frac{\partial^{3} R_{k}\left(\boldsymbol{\alpha}^{0}\right)}{\partial \alpha_{j 1} \partial \alpha_{j 2} \partial \alpha_{j 3}} \frac{\partial R_{l}\left(\boldsymbol{\alpha}^{0}\right)}{\partial \alpha_{j 4}} \frac{\partial R_{m}\left(\boldsymbol{\alpha}^{0}\right)}{\partial \alpha_{j 5}} \frac{\partial R_{n}\left(\boldsymbol{\alpha}^{0}\right)}{\partial \alpha_{j 6}} \\
& \left.\times\left(s_{j 1 j 2 j 3 j 4 j 5 j 6}-t_{j 1 j 2 j 3} t_{j 4 j 5 j 6}\right)\right] \sigma_{j 1} \sigma_{j 2} \sigma_{j 3} \sigma_{j 4} \sigma_{j 5} \sigma_{j 6} .
\end{aligned}
$$


B. The quadruple-correlations denoted as $\mu_{4}\left(\alpha_{i}, R_{j}, R_{k}, R_{\ell}\right)$, among one parameter, $\alpha_{i}$, and three responses $R_{j}(\boldsymbol{\alpha}), R_{k}(\boldsymbol{\alpha})$, and $R_{\ell}(\boldsymbol{\alpha})$, are defined as follows:

$\mu_{4}\left(\alpha_{i}, R_{j}, R_{k}, R_{\ell}\right) \triangleq\left\langle\left(\alpha_{i}-\alpha_{i}^{0}\right)\left[R_{j}(\boldsymbol{\alpha})-E\left(R_{j}\right)\right]\left[R_{k}(\boldsymbol{\alpha})-E\left(R_{k}\right)\right]\left[R_{\ell}(\boldsymbol{\alpha})-E\left(R_{\ell}\right)\right]\right\rangle_{\alpha}$.

1. If first-and second-order response sensitivities are available, the following 2nd-order approximate expression, denoted as $\mu_{4}^{(2)}\left(\alpha_{i}, R_{j}, R_{k}, R_{\ell}\right)$, can be obtained:

$$
\begin{aligned}
& \mu_{4}^{(2)}\left(\alpha_{i}, R_{j}, R_{k}, R_{\ell}\right)=\sum_{j 1=1}^{T P} \sum_{j 2=1}^{T P} \sum_{j 3=1}^{T P} \frac{\partial R_{j}\left(\boldsymbol{\alpha}^{0}\right)}{\partial \alpha_{j 1}} \frac{\partial R_{k}\left(\boldsymbol{\alpha}^{0}\right)}{\partial \alpha_{j 2}} \frac{\partial R_{l}\left(\boldsymbol{\alpha}^{0}\right)}{\partial \alpha_{j 3}} q_{j 1 j 2 j 3 i} \sigma_{j 1} \sigma_{j 2} \sigma_{j 3} \sigma_{i} \\
& +\frac{1}{2} \sum_{j 1=1}^{T P} \sum_{j 2=1}^{T P} \sum_{j 3=1}^{T P} \sum_{j 4=1}^{T P}\left[\frac{\partial R_{j}\left(\boldsymbol{\alpha}^{0}\right)}{\partial \alpha_{j 1}} \frac{\partial R_{k}\left(\boldsymbol{\alpha}^{0}\right)}{\partial \alpha_{j 2}} \frac{\partial^{2} R_{l}\left(\boldsymbol{\alpha}^{0}\right)}{\partial \alpha_{j 3} \partial \alpha_{j 4}}\left(p_{j 1 j 2 j 3 j 4 i}-t_{j 1 j 2 i} \rho_{j 3 j 4}\right)\right. \\
& +\frac{\partial R_{j}\left(\boldsymbol{\alpha}^{0}\right)}{\partial \alpha_{j 1}} \frac{\partial^{2} R_{k}\left(\boldsymbol{\alpha}^{0}\right)}{\partial \alpha_{j 2} \partial \alpha_{j 3}} \frac{\partial R_{l}\left(\boldsymbol{\alpha}^{0}\right)}{\partial \alpha_{j 4}}\left(p_{j 1 j 2 j 3 j 4 i}-\rho_{j 2 j 3} t_{j 1 j 4 i}\right)+\frac{\partial^{2} R_{j}\left(\boldsymbol{\alpha}^{0}\right)}{\partial \alpha_{j 1} \partial \alpha_{j 2}} \frac{\partial R_{l}\left(\boldsymbol{\alpha}^{0}\right)}{\partial \alpha_{j 3}} \frac{\partial R_{l}\left(\boldsymbol{\alpha}^{0}\right)}{\partial \alpha_{j 4}} \\
& \left.\times\left(p_{j 1 j 2 j 3 j 4 i}-\rho_{j 1 j 2} t_{j 3 j 4 i}\right)\right] \sigma_{j 1} \sigma_{j 2} \sigma_{j 3} \sigma_{j 4} \sigma_{i}+\frac{1}{4} \sum_{j 1=1}^{T P} \sum_{j 2} \sum_{j 3}^{T P} \sum_{j 4}^{T P} \sum_{j 5}^{T P}\left[\frac{\partial R_{j}\left(\boldsymbol{\alpha}^{0}\right)}{\partial \alpha_{j 1}} \frac{\partial^{2} R_{k}\left(\boldsymbol{\alpha}^{0}\right)}{\partial \alpha_{j 2} \partial \alpha_{j 3}} \frac{\partial^{2} R_{l}\left(\boldsymbol{\alpha}^{0}\right)}{\partial \alpha_{j 4} \partial \alpha_{j 5}}\right. \\
& \times\left(s_{j 1 j 2 j 3 j 4 j 5 i}-\rho_{j 2 j 3} q_{j 1 j 4 j 5 i}-q_{j 1 j 2 j 3 i} \rho_{j 4 j 5}+\rho_{j 1 i} \rho_{j 2 j 3} \rho_{j 4 j 5}\right)+\frac{\partial^{2} R_{j}\left(\boldsymbol{\alpha}^{0}\right)}{\partial \alpha_{j 1} \partial \alpha_{j 2}} \frac{\partial R_{l}\left(\boldsymbol{\alpha}^{0}\right)}{\partial \alpha_{j 3}} \frac{\partial^{2} R_{l}\left(\boldsymbol{\alpha}^{0}\right)}{\partial \alpha_{j 4} \partial \alpha_{j 5}} \\
& \times\left(s_{j 1 j 2 j 3 j 4 j 5 i}-\rho_{j 1 j 2} q_{j 3 j 4 j 5 i}-q_{j 1 j 2 j 3 i} \rho_{j 4 j 5}+\rho_{j 1 j 2} \rho_{j 3 i} \rho_{j 4 j 5}\right)+\frac{\partial^{2} R_{j}\left(\boldsymbol{\alpha}^{0}\right)}{\partial \alpha_{j 1} \partial \alpha_{j 2}} \frac{\partial^{2} R_{k}\left(\boldsymbol{\alpha}^{0}\right)}{\partial \alpha_{j 3} \partial \alpha_{j 4}} \frac{\partial R_{l}\left(\boldsymbol{\alpha}^{0}\right)}{\partial \alpha_{j 5}} \\
& \left.\times\left(s_{j 1 j 2 j 3 j 4 j 5 i}-\rho_{j 1 j 2} q_{j 3 j 4 j 5 i}-q_{j 1 j 2 j 5 i} \rho_{j 3 j 4}+\rho_{j 1 j 2} \rho_{j 3 j 4} \rho_{j 5 i}\right)\right] \sigma_{j 1} \sigma_{j 2} \sigma_{j 3} \sigma_{j 4} \sigma_{j 5} \sigma_{i} .
\end{aligned}
$$

2. If third-order response sensitivities are also available, the following 3rd-order approximate expression, denoted as $\mu_{4}^{(3)}\left(\alpha_{i}, R_{j}, R_{k}, R_{\ell}\right)$, which contains all of the 6th-order terms in the standard deviations, can be obtained:

$$
\begin{aligned}
& \mu_{4}^{(3)}\left(\alpha_{i}, R_{j}, R_{k}, R_{\ell}\right)=\frac{1}{6} \sum_{j 1=1}^{T P} \sum_{j 2=1}^{T P} \sum_{j 3=1}^{T P} \sum_{j 4=1}^{T P} \sum_{j 5=1}^{T P}\left[\frac{\partial R_{j}\left(\boldsymbol{\alpha}^{0}\right)}{\partial \alpha_{j 1}} \frac{\partial R_{k}\left(\boldsymbol{\alpha}^{0}\right)}{\partial \alpha_{j 2}} \frac{\partial^{3} R_{l}\left(\boldsymbol{\alpha}^{0}\right)}{\partial \alpha_{j 3} \partial \alpha_{j 4} \partial \alpha_{j 5}}\right. \\
& \times\left(s_{j 1 j 2 j 3 j 4 j 5 i}-t_{j 1 j 2 i} t_{j 3 j 4 j 5}\right)+\frac{\partial R_{j}\left(\boldsymbol{\alpha}^{0}\right)}{\partial \alpha_{j 1}} \frac{\partial^{3} R_{k}\left(\boldsymbol{\alpha}^{0}\right)}{\partial \alpha_{j 2} \partial \alpha_{j 3} \partial \alpha_{j 4}} \frac{\partial R_{l}\left(\boldsymbol{\alpha}^{0}\right)}{\partial \alpha_{j 5}} \\
& \times\left(s_{j 1 j 2 j 3 j 4 j 5 i}-t_{j 1 j 5 i} t_{j 2 j 3 j 4}\right)+\frac{\partial^{3} R_{k}\left(\boldsymbol{\alpha}^{0}\right)}{\partial \alpha_{j 1} \partial \alpha_{j 2} \partial \alpha_{j 3}} \frac{\partial R_{k}\left(\boldsymbol{\alpha}^{0}\right)}{\partial \alpha_{j 4}} \frac{\partial R_{l}\left(\boldsymbol{\alpha}^{0}\right)}{\partial \alpha_{j 5}} \\
& \left.\times\left(s_{j 1 j 2 j 3 j 4 j 5 i}-t_{j 1 j 2 j 3} t_{j 4 j 5 i}\right)\right] \sigma_{j 1} \sigma_{j 2} \sigma_{j 3} \sigma_{j 4} \sigma_{j 5} \sigma_{i}+\mu_{4}^{(2)}\left(\alpha_{i}, R_{j}, R_{k}, R_{\ell}\right) .
\end{aligned}
$$

C. The quadruple-correlations, denoted as $\mu_{4}\left(\alpha_{i}, \alpha_{j}, R_{k}, R_{\ell}\right)$, among two parameters $\alpha_{i}, \alpha_{j}$ and two responses $R_{k}(\alpha), R_{\ell}(\alpha)$, are defined as follows:

$$
\begin{aligned}
& \mu_{4}\left(\alpha_{i}, \alpha_{j}, R_{k}, R_{\ell}\right) \triangleq\left\langle\left(\alpha_{i}-\alpha_{i}^{0}\right)\left(\alpha_{j}-\alpha_{j}^{0}\right)\left[R_{k}(\boldsymbol{\alpha})-E\left(R_{k}\right)\right]\left[R_{\ell}(\boldsymbol{\alpha})-E\left(R_{\ell}\right)\right]\right\rangle_{\alpha} \\
& =\sum_{j 1=1}^{T P} \sum_{j 2=1}^{T P} \frac{\partial R_{k}\left(\boldsymbol{\alpha}^{0}\right)}{\partial \alpha_{j 1}} \frac{\partial R_{l}\left(\boldsymbol{\alpha}^{0}\right)}{\partial \alpha_{j 2}} q_{j 1 j 2 i j} \sigma_{j 1} \sigma_{j 2} \sigma_{i} \sigma_{j} \\
& +\frac{1}{2} \sum_{j 1=1}^{T P} \sum_{j 2=1}^{T P} \sum_{j 3=1}^{T P}\left[\frac{\partial R_{k}\left(\boldsymbol{\alpha}^{0}\right)}{\partial \alpha_{j 1}} \frac{\partial^{2} R_{l}\left(\boldsymbol{\alpha}^{0}\right)}{\partial \alpha_{j 2} \partial \alpha_{j 3}}\left(p_{j 1 j 2 j 3 i j}-t_{j 1 i j} \rho_{j 2 j 3}\right)\right. \\
& \left.+\frac{\partial^{2} R_{k}\left(\boldsymbol{\alpha}^{0}\right)}{\partial \alpha_{j 1} \partial \alpha_{j 2}} \frac{\partial R_{l}\left(\boldsymbol{\alpha}^{0}\right)}{\partial \alpha_{j 3}}\left(p_{j 1 j 2 j 3 i j}-\rho_{j 1 j 2} t_{j 3 i j}\right)\right] \sigma_{j 1} \sigma_{j 2} \sigma_{j 3} \sigma_{i} \sigma_{j} \\
& +\frac{1}{4} \sum_{j 1=1}^{T P} \sum_{j 2=1}^{T P} \sum_{j 3=1}^{T P} \sum_{j 4=1}^{T P} \frac{\partial^{2} R_{k}\left(\boldsymbol{\alpha}^{0}\right)}{\partial \alpha_{j 1} \partial \alpha_{j 2}} \frac{\partial^{2} R_{l}\left(\boldsymbol{\alpha}^{0}\right)}{\partial \alpha_{j 3} \partial \alpha_{j 4}} \\
& \times\left(s_{j 1 j 2 j 3 j 4 i j}+\rho_{j 1 j 2} \rho_{j 3 j 4} \rho_{i j}-\rho_{j 1 j 2} q_{j 3 j 4 i j}-q_{j 1 j 2 i j} \rho_{j 3 j 4}\right) \sigma_{j 1} \sigma_{j 2} \sigma_{j 3} \sigma_{j 4} \sigma_{i} \sigma_{j} \\
& +\frac{1}{6} \sum_{j 1=1}^{T P} \sum_{j 2=1}^{T P} \sum_{j 3=1}^{T P} \sum_{j 4=1}^{T P}\left[\frac{\partial R_{k}\left(\boldsymbol{\alpha}^{0}\right)}{\partial \alpha_{j 1}} \frac{\partial^{3} R_{l}\left(\boldsymbol{\alpha}^{0}\right)}{\partial \alpha_{j 2} \partial \alpha_{j 3} \partial \alpha_{j 4}}\left(s_{j 1 j 2 j 3 j 4 i j}-t_{j 1 i j} t_{j 2} 3 j 4\right)\right. \\
& \left.+\frac{\partial^{3} R_{k}\left(\boldsymbol{\alpha}^{0}\right)}{\partial \alpha_{j 1} \partial \alpha_{j 2} \partial \alpha_{j 3}} \frac{\partial R_{l}\left(\boldsymbol{\alpha}^{0}\right)}{\partial \alpha_{j 4}}\left(s_{j 1 j 2 j 3 j 4 i j}-t_{j 1 j 2 j 3} t_{j 4 i j}\right)\right] \sigma_{j 1} \sigma_{j 2} \sigma_{j 3} \sigma_{j 4} \sigma_{i} \sigma_{j} .
\end{aligned}
$$

D. The quadruple-correlations, denoted as $\mu_{4}\left(\alpha_{i}, \alpha_{j}, \alpha_{k}, R_{\ell}\right)$, among three parameters $\alpha_{i}, \alpha_{j}, \alpha_{k}$, and one response, $R_{\ell}(\alpha)$, are defined as follows: 


$$
\begin{aligned}
& \mu_{4}\left(\alpha_{i}, \alpha_{j}, \alpha_{k}, R_{\ell}\right) \triangleq\left\langle\left(\alpha_{i}-\alpha_{i}^{0}\right)\left(\alpha_{j}-\alpha_{j}^{0}\right)\left(\alpha_{k}-\alpha_{k}^{0}\right)\left[R_{\ell}(\boldsymbol{\alpha})-E\left(R_{\ell}\right)\right]\right\rangle_{\alpha} \\
& =\sum_{i_{1}=1}^{T P} \frac{\partial R_{\ell}\left(\boldsymbol{\alpha}^{0}\right)}{\partial \alpha_{j 1}} q_{j 1 i j k} \sigma_{j 1} \sigma_{i} \sigma_{j} \sigma_{k}+\frac{1}{2} \sum_{j 1=1}^{T P} \sum_{j 2=1}^{T P} \frac{\partial^{2} R_{\ell}\left(\boldsymbol{\alpha}^{0}\right)}{\partial \alpha_{j 1} \partial \alpha_{j 2}}\left(p_{j 1 j 2 i j k}-\rho_{j 1 j 2} t_{i j k}\right) \sigma_{j 1} \sigma_{j 2} \sigma_{i} \sigma_{j} \sigma_{k} \\
& +\frac{1}{6} \sum_{j 1=1}^{T P} \sum_{j 2=1}^{T P} \sum_{j 3=1}^{T P} \frac{\partial^{3} R_{\ell}\left(\boldsymbol{\alpha}^{0}\right)}{\partial \alpha_{j 1} \partial \alpha_{j 2} \partial \alpha_{j 3}}\left(s_{j 1 j 2 j 3 i j k}-t_{j 1 j 2 j 3} t_{i j k}\right) \sigma_{j 1} \sigma_{j 2} \sigma_{j 3} \sigma_{i} \sigma_{j} \sigma_{k} .
\end{aligned}
$$

The formulas presented in this Section are consistent up to and including the 6th-order standard deviations, thus generalizing the formulas presented by Tukey [49]. It is essential to obtaining the exact and complete set of first-order sensitivities of responses to model parameters, since these sensitivities contribute the leading terms to the second-, third-, and fourth-order moments of the response distribution. The second-order sensitivities contribute the leading correction terms to the response's expected value and to the response's skewness. In particular, the skewness, denoted as $\gamma_{1}(R)$, of a response $R$ is defined as $\gamma_{1}(R)=\mu_{3}(R) /[\operatorname{var}(R)]^{\frac{3}{2}}$, where $\mu_{3}(R)$ denotes the third central moment of the response distribution. Hence, a "first-order sensitivity and uncertainty quantification" will always produce an erroneous third moment (and hence skewness) of the predicted response distribution, unless the unknown response distribution happens to be symmetrical. At least second-order sensitivities must be used in order to estimate the third-order moment (and hence the skewness) of the response distribution. Skewness indicates the direction and relative magnitude of a distribution's deviation from the normal distribution while kurtosis indicates the propensity of the predicted response distribution to have heavy tails and/or outliers. With pronounced skewness, standard statistical inference procedures such as constructing a confidence interval for the mean (expectation) of a computed/predicted model response will be not only incorrect, in the sense that the true coverage level will differ from the nominal (e.g., 95\%) level, but the error probabilities will be unequal on each side of the predicted mean.

\subsection{Illustrating the Need for High-Order Uncertainty Quantification: Neutron Scattering in an} Infinite Hydrogenous Medium

If a standard deviation is not provided for the measurement of $\sigma_{s}\left(E_{m}\right)$, then the most conservative assumption is to consider that $\sigma_{S}(E)$ is uniformly distributed within the interval provided in Equation (17), in which the Taylor-series of $\Phi_{H}(\sigma ; E)$ convergences, since the uniform distribution is the least informative (hence, the least biased) distribution. It will henceforth be assumed that the distribution for $\sigma_{S}(E)$ is uniform in the interval given in Equation (17). Thus, the uniform probability distribution, $P\left[\sigma_{S}(E)\right]$, the expectation, $\mathbf{E}\left[\sigma_{S}(E)\right]$, the variance, $\operatorname{Var}\left[\sigma_{S}(E)\right]$, and the standard deviation, $S D\left[\sigma_{\mathcal{S}}(E)\right]$, of $\sigma_{S}(E)$ have the following expressions:

$$
\begin{aligned}
& P\left[\sigma_{S}(E)\right]=\frac{1}{2 \beta \sigma_{m}} ; \quad \mathbf{E}\left[\sigma_{\mathcal{S}}(E)\right]=\sigma_{m} ; \quad \operatorname{Var}\left[\sigma_{\mathcal{S}}(E)\right]=\frac{\left(\beta \sigma_{m}\right)^{2}}{3} ; \\
& S D\left[\sigma_{\mathcal{S}}(E)\right]=\frac{1}{\sqrt{3}} \beta \sigma_{m} ; \quad 0<\beta<1 .
\end{aligned}
$$

The third and fourth moments, $\mu_{3}\left[\sigma_{S}(E)\right]$ and $\mu_{4}\left[\sigma_{S}(E)\right]$, of the uniform distribution $\sigma_{S}(E)$ have the following expressions:

$$
\begin{aligned}
& \mu_{3}\left[\sigma_{S}(E)\right] \triangleq \int_{(1-\beta) \sigma_{m}}^{(1+\beta) \sigma_{m}}\left[\sigma_{\mathcal{S}}(E)-\sigma_{m}\right]^{3} d \sigma_{S}(E)=0 \\
& \mu_{4}\left[\sigma_{S}(E)\right] \triangleq \int_{(1-\beta) \sigma_{m}}^{(1+\beta) \sigma_{m}}\left[\sigma_{S}(E)-\sigma_{m}\right]^{4} d \sigma_{S}(E)=\frac{\left(\beta \sigma_{m}\right)^{4}}{5}
\end{aligned}
$$

The uniform distribution defined in Equation (94) is symmetrical with respect to $\sigma_{m}$ and can be visualized as a rectangle of height $P\left[\sigma_{s}(E)\right]=\left[2 \beta \sigma_{m}\right]^{-1}$ and width extending from $(1-\beta) \sigma_{m}$ to $(1+\beta) \sigma_{m}$ for $0<\beta<1$. When $\beta$ increases towards unity, the uniform 
distribution $P\left[\sigma_{s}(E)\right]=\left[2 \beta \sigma_{m}\right]^{-1}$ widens and its height decreases towards $0.5 / \sigma_{m}$. At the other extreme, when $\beta$ decreases towards zero, the distribution $P\left[\sigma_{S}(E)\right]=\left[2 \beta \sigma_{m}\right]^{-1}$ becomes very narrow and peaked, tending towards a Dirac-delta distribution centered at $\sigma_{m}$.

It follows from the expressions provided in Equations (94) and (17) that, if $\sigma_{\mathcal{S}}(E)$ is distributed uniformly as shown in Equation (94), then the Taylor-series of shown in Equation (15) for $\Phi_{H}(\sigma ; E)$ converges only in the following interval expressed in terms of "standard deviations of $\sigma_{\mathcal{S}}(E)$ ":

$$
\sigma_{m}-\sqrt{3} \cdot S D\left[\sigma_{s}(E)\right] \leq \sigma_{S}(E) \leq \sigma_{m}+\sqrt{3} \cdot S D\left[\sigma_{s}(E)\right] ; 0<\beta<1 .
$$

The expectation, $\mathbf{E}\left[\Phi_{H}\right]$, of the flux $\Phi_{H}(\sigma ; E)$, is obtained by integrating Equation (15) over the uniform probability distribution for $\sigma_{S}(E)$ shown in Equation (94). The result of this integration is:

$$
\begin{aligned}
& \mathbf{E}\left[\Phi_{H}\right]=\frac{Q\left(\boldsymbol{\alpha}^{0}\right)}{E N_{H}} \frac{1}{2 \beta \sigma_{m}} \int_{(1-\beta) \sigma_{m}}^{(1+\beta) \sigma_{m}} \frac{d \sigma_{s}(E)}{\sigma_{s}(E)}=\frac{Q\left(\boldsymbol{\alpha}^{0}\right)}{E N_{H}} \frac{1}{2 \beta \sigma_{m}} \ln \frac{1+\beta}{1-\beta} \\
& =\frac{Q\left(\boldsymbol{\alpha}^{0}\right)}{E N_{H} \sigma_{m}} \sum_{n=0}^{\infty} \frac{1}{2 \beta \sigma_{m}} \int_{(1-\beta) \sigma_{m}}^{(1+\beta) \sigma_{m}}\left[\frac{\sigma_{s}(E)-\sigma_{m}}{\sigma_{m}}\right]^{n} d \sigma_{S}(E)=\frac{Q\left(\boldsymbol{\alpha}^{0}\right)}{E N_{H} \sigma_{m}} \sum_{k=0}^{\infty} \frac{\beta^{2 k}}{2 k+1} \\
& =\frac{Q\left(\boldsymbol{\alpha}^{0}\right)}{E N_{H}\left(\beta \sigma_{m}\right)} \operatorname{Arctanh}(\beta)=\frac{Q\left(\boldsymbol{\alpha}^{0}\right)}{E N_{H}} \frac{1}{2 \beta \sigma_{m}} \ln \frac{1+\beta}{1-\beta} ; 0<\beta<1 .
\end{aligned}
$$

As indicated in (97), $\mathbf{E}\left[\Phi_{H}\right]$ is an even function of $\beta$, i.e.,

$$
\mathbf{E}\left[\Phi_{H}, \beta\right]=\mathbf{E}\left[\Phi_{H},-\beta\right]=\frac{Q\left(\alpha^{0}\right)}{E N_{H}} \frac{1}{2 \beta \sigma_{m}} \ln \frac{1+\beta}{1-\beta} .
$$

The $n^{\text {th }}$-order approximation, denoted as $\mathbf{E}^{(n)}\left[\Phi_{H}\right]$, of the expectation $\mathbf{E}\left[\Phi_{H}\right]$, is provided by the following truncated Taylor series:

$$
\mathbf{E}^{(2 n)}\left[\Phi_{H}\right]=\mathbf{E}\left[\Phi_{H}^{(2 n)}(\sigma ; E)\right]=\frac{Q\left(\alpha^{0}\right)}{E N_{H} \sigma_{m}} \sum_{k=0}^{n} \frac{\beta^{2 k}}{2 k+1} ; \mathbf{E}^{(2 n+1)}\left[\Phi_{H}\right]=\mathbf{E}^{(2 n)}\left[\Phi_{H}\right] ; n=0,1, \ldots ;
$$

Notably, the convergence of the infinite series-representation of $\mathbf{E}\left[\Phi_{H}\right]$ obtained in Equation (97) depends solely on the chosen value for the positive parameter $\beta$ : the smaller the chosen value for $\beta$, the faster the convergence of the series representation for $\mathbf{E}\left[\Phi_{H}\right]$.

The variance, denoted as $V\left[\Phi_{H}\right]$, of the flux $\Phi_{H}(\sigma ; E)$, is obtained by recalling the following relation:

$$
V\left[\Phi_{H}\right]=\mathbf{E}\left[\left(\Phi_{H}\right)^{2}\right]-\mathbf{E}^{2}\left[\Phi_{H}\right]
$$

Using Equations and (97) in Equation (100) yields the following result:

$$
\begin{gathered}
V\left[\Phi_{H}\right]=\left[\frac{Q\left(\boldsymbol{\alpha}^{0}\right)}{E N_{H}}\right]^{2} \frac{1}{2 \beta \sigma_{m}} \int_{(1-\beta) \sigma_{m}}^{(1+\beta) \sigma_{m}} \frac{d \sigma_{s}(E)}{\left[\sigma_{s}(E)\right]^{2}}-\left[\frac{Q\left(\boldsymbol{\alpha}^{0}\right)}{E N_{H}} \frac{1}{2 \beta \sigma_{m}} \ln \frac{1+\beta}{1-\beta}\right]^{2} \\
=\left[\frac{Q\left(\boldsymbol{\alpha}^{0}\right)}{E N_{H} \sigma_{m}}\right]^{2}\left[\frac{1}{1-\beta^{2}}-\frac{1}{4 \beta^{2}}\left(\ln \frac{1+\beta}{1-\beta}\right)^{2}\right]
\end{gathered}
$$

Note that $V\left[\Phi_{H}\right]$ is an even function of $\beta$, i.e.,

$$
V\left[\Phi_{H}, \beta\right]=V\left[\Phi_{H},-\beta\right]=\left[\frac{Q\left(\alpha^{0}\right)}{E N_{H} \sigma_{m}}\right]^{2}\left[\frac{1}{1-\beta^{2}}-\frac{1}{4 \beta^{2}}\left(\ln \frac{1+\beta}{1-\beta}\right)^{2}\right]
$$

As indicated by the result obtained in Equation (101), the convergence of the seriesrepresentation of $V\left[\Phi_{H}\right]$ would also depend solely on the chosen value for the positive 
parameter $\beta$ : the smaller the chosen value for $\beta$, the faster would be the convergence of the series representation for $V\left[\Phi_{H}\right]$.

The $n^{\text {th }}$-order approximation, denoted as $V^{(n)}\left[\Phi_{H}\right]$, of the variance $V\left[\Phi_{H}\right]$, is also obtained by using the relation provided in Equation (100), which takes on the following form:

$$
V^{(n)}\left[\Phi_{H}\right]=\mathbf{E}^{(n)}\left[\left(\Phi_{H}^{(n)}\right)^{2}\right]-\left(\mathbf{E}^{(n)}\right)^{2}\left[\Phi_{H}^{(n)}\right] ; n=1,2, \ldots ;
$$

The third-order moment, denoted as $\mu_{3}\left[\Phi_{H}\right]$, of the flux $\Phi_{H}(\sigma ; E)$, is obtained using Equations (15) and (97) in the definition of $\mu_{3}\left[\Phi_{H}\right]$ and performing the ensuing integrations, to obtain the result shown below:

$$
\begin{aligned}
& \mu_{3}\left[\Phi_{H}\right] \triangleq \frac{1}{2 \beta \sigma_{m}} \int_{(1-\beta) \sigma_{m}}^{(1+\beta) \sigma_{m}}\left\{\Phi_{H}(\sigma ; E)-\mathbf{E}\left[\Phi_{H}\right]\right\}^{3} d \sigma_{s}(E) \\
& =\left[\frac{Q\left(\boldsymbol{\alpha}^{0}\right)}{E N_{H} \sigma_{m}}\right]^{3} \frac{1}{2 \beta}\left[\frac{2 \beta}{\left(1-\beta^{2}\right)^{2}}-\frac{3}{1-\beta^{2}} \ln \frac{1+\beta}{1-\beta}+\frac{1}{2 \beta^{2}}\left(\ln \frac{1+\beta}{1-\beta}\right)^{3}\right] .
\end{aligned}
$$

Note that $\mu_{3}\left[\Phi_{H}\right]$ is an even function of $\beta$, i.e.,

$$
\begin{gathered}
\mu_{3}\left[\Phi_{H}, \beta\right]=\mu_{3}\left[\Phi_{H},-\beta\right] \\
=\left[\frac{Q\left(\alpha^{0}\right)}{E N_{H} \sigma_{m}}\right]^{3} \frac{1}{2 \beta}\left[\frac{2 \beta}{\left(1-\beta^{2}\right)^{2}}-\frac{3}{1-\beta^{2}} \ln \frac{1+\beta}{1-\beta}+\frac{1}{2 \beta^{2}}\left(\ln \frac{1+\beta}{1-\beta}\right)^{3}\right] .
\end{gathered}
$$

The skewness of the distribution of the response $\Phi_{H}(\sigma ; E)$ will be denoted as $S k e w\left[\Phi_{H}\right]$ and is defined as follows:

$$
\operatorname{Skew}\left[\Phi_{H}\right]=\frac{\mu_{3}\left[\Phi_{H}\right]}{\left\{V\left[\Phi_{H}\right]\right\}^{\frac{3}{2}}}=\frac{\frac{1}{2 \beta}\left[\frac{2 \beta}{\left(1-\beta^{2}\right)^{2}}-\frac{3}{1-\beta^{2}} \ln \frac{1+\beta}{1-\beta}+\frac{1}{2 \beta^{2}}\left(\ln \frac{1+\beta}{1-\beta}\right)^{3}\right]}{\left[\frac{1}{1-\beta^{2}}-\frac{1}{4 \beta^{2}}\left(\ln \frac{1+\beta}{1-\beta}\right)^{2}\right]^{\frac{3}{2}}} .
$$

Skewness indicates the direction and relative magnitude of a distribution's deviation from the normal distribution. Note that, for this illustrative model, $\operatorname{Skew}\left[\Phi_{H}, \beta\right]=$ Skew $\left[\Phi_{H},-\beta\right]$.

The $n^{\text {th }}$-order approximation of the third-order moment, denoted as $\mu_{3}^{(n)}\left[\Phi_{H}\right]$, is defined below:

$$
\mu_{3}^{(n)}\left[\Phi_{H}\right] \triangleq \frac{1}{2 \beta \sigma_{m}} \int_{(1-\beta) \sigma_{m}}^{(1+\beta) \sigma_{m}}\left\{\Phi_{H}^{(n)}(\sigma ; E)-\mathbf{E}^{(n)}\left[\Phi_{H}\right]\right\}^{3} d \sigma_{\mathcal{S}}(E) .
$$

It follows from Equations (107), (16) and (99) that the first-order approximation of the third-order moment, denoted as $\mu_{3}^{(1)}\left[\Phi_{H}\right]$, has the following expression:

$$
\begin{aligned}
& \mu_{3}^{(1)}\left[\Phi_{H}\right] \triangleq \frac{1}{2 \beta \sigma_{m}} \int_{(1-\beta) \sigma_{m}}^{(1+\beta) \sigma_{m}}\left\{\Phi_{H}^{(1)}(\sigma ; E)-\mathbf{E}^{(1)}\left[\Phi_{H}\right]\right\}^{3} d \sigma_{s}(E) \\
& =\frac{1}{2 \beta \sigma_{m}} \int_{(1-\beta) \sigma_{m}}^{(1+\beta) \sigma_{m}}\left\{\frac{Q\left(\boldsymbol{\alpha}^{0}\right)}{E N_{H} \sigma_{m}}\left[1-\frac{\sigma_{s}(E)-\sigma_{m}}{\sigma_{m}}\right]-\frac{Q\left(\boldsymbol{\alpha}^{0}\right)}{E N_{H} \sigma_{m}}\right\}^{3} d \sigma_{s}(E) \equiv 0 .
\end{aligned}
$$

It also follows from Equations (107), (16) and (99) that the second-order approximation of the third-order moment, denoted as $\mu_{3}^{(2)}\left[\Phi_{H}\right]$, has the following expression: 


$$
\begin{aligned}
& \mu_{3}^{(2)}\left[\Phi_{H}\right] \triangleq \frac{1}{2 \beta \sigma_{m}} \int_{(1-\beta) \sigma_{m}}^{(1+\beta) \sigma_{m}}\left\{\Phi_{H}^{(2)}(\sigma ; E)-\mathbf{E}^{(2)}\left[\Phi_{H}\right]\right\}^{3} d \sigma_{S}(E) \\
& =\frac{1}{2 \beta \sigma_{m}} \int_{(1-\beta) \sigma_{m}}^{(1+\beta) \sigma_{m}}\left\{\frac{Q\left(\boldsymbol{\alpha}^{0}\right)}{E N_{H} \sigma_{m}}\left[1-\frac{\sigma_{s}(E)-\sigma_{m}}{\sigma_{m}}+\left(\frac{\sigma_{S}(E)-\sigma_{m}}{\sigma_{m}}\right)^{2}\right]-\frac{Q\left(\boldsymbol{\alpha}^{0}\right)}{E N_{H} \sigma_{m}}\left(1+\frac{\beta^{2}}{3}\right)\right\}^{3} d \sigma_{S}(E) \\
& =\left[\frac{Q\left(\boldsymbol{\alpha}^{0}\right)}{E N_{H} \sigma_{m}}\right]^{3} \frac{4}{15} \beta^{4}+O\left(\beta^{6}\right) .
\end{aligned}
$$

It is evident that high-order approximations for the $3^{\text {rd }}$-order moment are very laborious to obtain. On the other hand, since the definition of skewness involves not only the response's third moment but also the response's variance, it is possible to compute approximate values of the skewness, denoted as $\operatorname{Skew}{ }^{(m, n)}\left[\Phi_{H}\right]$, by using approximations of different orders for the response's variance and third moment, respectively, as follows:

$$
\operatorname{Skew}^{(m, n)}\left[\Phi_{H}\right] \triangleq \frac{\mu_{3}^{(m)}\left[\Phi_{H}\right]}{\left\{V^{(n)}\left[\Phi_{H}\right]\right\}^{\frac{3}{2}}} ; m, n=1,2, \ldots
$$

The flexible definition for the approximate skewness $S k e w^{(m, n)}\left[\Phi_{H}\right]$ provided in Equation (110) enables the use of higher-order approximations for the response variance, which require less computational effort to determine by comparison to the same order of approximation for the third moment. The flexible definition of $S k e w^{(m, n)}\left[\Phi_{H}\right]$ could provide higher accuracy for the skewness with less computational effort by using a lower-order approximation for the third-moment than the order of approximation used for computing the variance.

The fourth-order moment, denoted as $\mu_{4}\left[\Phi_{H}\right]$, of the flux $\Phi_{H}(\sigma ; E)$, is defined as follows:

$$
\begin{aligned}
& \mu_{4}\left[\Phi_{H}\right] \triangleq \frac{1}{2 \beta \sigma_{m}} \int_{(1-\beta) \sigma_{m}}^{(1+\beta) \sigma_{m}}\left\{\Phi_{H}(\sigma ; E)-\mathbf{E}\left[\Phi_{H}\right]\right\}^{4} d \sigma_{S}(E) \\
& =\left[\frac{Q\left(\alpha^{0}\right)}{E N_{H} \sigma_{m}}\right]^{4}\left[\frac{1+\beta^{2} / 3}{\left(1-\beta^{2}\right)^{3}}-\frac{2}{\beta\left(1-\beta^{2}\right)^{2}} \ln \frac{1+\beta}{1-\beta}+\frac{3}{2 \beta^{2}\left(1-\beta^{2}\right)}\left(\ln \frac{1+\beta}{1-\beta}\right)^{2}-\frac{3}{16 \beta^{4}}\left(\ln \frac{1+\beta}{1-\beta}\right)^{4}\right] .
\end{aligned}
$$

Note that $\mu_{4}\left[\Phi_{H}\right]$ is an even function of $\beta$, i.e.,

$$
\begin{gathered}
\mu_{4}\left[\Phi_{H}, \beta\right]=\mu_{4}\left[\Phi_{H},-\beta\right] \\
=\left[\frac{Q\left(\alpha^{0}\right)}{E N_{H} \sigma_{m}}\right]^{4}\left[\frac{1+\beta^{2} / 3}{\left(1-\beta^{2}\right)^{3}}-\frac{2}{\beta\left(1-\beta^{2}\right)^{2}} \ln \frac{1+\beta}{1-\beta}+\frac{3}{2 \beta^{2}\left(1-\beta^{2}\right)}\left(\ln \frac{1+\beta}{1-\beta}\right)^{2}-\frac{3}{16 \beta^{4}}\left(\ln \frac{1+\beta}{1-\beta}\right)^{4}\right] .
\end{gathered}
$$

The $n^{t h}$-order approximation of the fourth-order moment, denoted as $\mu_{4}^{(n)}\left[\Phi_{H}\right]$, is defined below:

$$
\mu_{4}^{(n)}\left[\Phi_{H}\right] \triangleq \frac{1}{2 \beta \sigma_{m}} \int_{(1-\beta) \sigma_{m}}^{(1+\beta) \sigma_{m}}\left\{\Phi_{H}^{(n)}(\sigma ; E)-\mathbf{E}^{(n)}\left[\Phi_{H}\right]\right\}^{4} d \sigma_{s}(E) .
$$

It follows from Equations (113), (16) and (99) that the first-order approximation of the fourth-order moment, denoted as $\mu_{4}^{(1)}\left[\Phi_{H}\right]$, has the following expression:

$$
\begin{aligned}
& \mu_{4}^{(1)}\left[\Phi_{H}\right] \triangleq \frac{1}{2 \beta \sigma_{m}} \int_{(1-\beta) \sigma_{m}}^{(1+\beta) \sigma_{m}}\left\{\Phi_{H}^{(1)}(\sigma ; E)-\mathbf{E}^{(1)}\left[\Phi_{H}\right]\right\}^{4} d \sigma_{S}(E) \\
& =\frac{1}{2 \beta \sigma_{m}} \int_{(1-\beta) \sigma_{m}}^{(1+\beta) \sigma_{m}}\left\{\frac{Q\left(\boldsymbol{\alpha}^{0}\right)}{E N_{H} \sigma_{m}}\left[1-\frac{\sigma_{s}(E)-\sigma_{m}}{\sigma_{m}}\right]-\frac{Q\left(\boldsymbol{\alpha}^{0}\right)}{E N_{H} \sigma_{m}}\right\}^{4} d \sigma_{S}(E)=\left[\frac{Q\left(\boldsymbol{\alpha}^{0}\right)}{E N_{H} \sigma_{m}}\right]^{4} \frac{\beta^{4}}{5} .
\end{aligned}
$$


It also follows from Equations (113), (16) and (99) that the second-order approximation of the third-order moment, denoted as $\mu_{4}^{(2)}\left[\Phi_{H}\right]$, has the following expression:

$$
\begin{aligned}
& \mu_{4}^{(2)}\left[\Phi_{H}\right] \triangleq \frac{1}{2 \beta \sigma_{m}} \int_{(1-\beta) \sigma_{m}}^{(1+\beta) \sigma_{m}}\left\{\Phi_{H}^{(2)}(\sigma ; E)-\mathbf{E}^{(2)}\left[\Phi_{H}\right]\right\}^{4} d \sigma_{s}(E) \\
& =\frac{1}{2 \beta \sigma_{m}} \int_{(1-\beta) \sigma_{m}}^{(1+\beta) \sigma_{m}}\left\{\frac{Q\left(\boldsymbol{\alpha}^{0}\right)}{E N_{H} \sigma_{m}}\left[1-\frac{\sigma_{s}(E)-\sigma_{m}}{\sigma_{m}}+\left(\frac{\sigma_{s}(E)-\sigma_{m}}{\sigma_{m}}\right)^{2}\right]-\frac{Q\left(\boldsymbol{\alpha}^{0}\right)}{E N_{H} \sigma_{m}}\left(1+\frac{\beta^{2}}{3}\right)\right\}^{4} d \sigma_{s}(E) \\
& =\left[\frac{Q\left(\boldsymbol{\alpha}^{0}\right)}{E N_{H} \sigma_{m}}\right]^{3}\left[\frac{\beta^{4}}{5}+\frac{6}{7} \beta^{6}+O\left(\beta^{8}\right)\right] .
\end{aligned}
$$

Evidently, high-order approximations for the fourth-order moment $\mu_{4}\left[\Phi_{H}\right]$ are very laborious to obtain.

The kurtosis of the distribution of the response $\Phi_{H}(\sigma ; E)$ will be denoted as $\operatorname{Kurt}\left[\Phi_{H}\right]$ and is defined as follows:

$$
\begin{aligned}
& \operatorname{Kurt}\left[\Phi_{H}\right]=\frac{\mu_{4}\left[\Phi_{H}\right]}{\left\{V\left[\Phi_{H}\right]\right\}^{2}} \\
& =\frac{\left[\frac{1+\beta^{2} / 3}{\left(1-\beta^{2}\right)^{3}}-\frac{2}{\beta\left(1-\beta^{2}\right)^{2}} \ln \frac{1+\beta}{1-\beta}+\frac{3}{2 \beta^{2}\left(1-\beta^{2}\right)}\left(\ln \frac{1+\beta}{1-\beta}\right)^{2}-\frac{3}{16 \beta^{4}}\left(\ln \frac{1+\beta}{1-\beta}\right)^{4}\right]}{\left[\frac{1}{1-\beta^{2}}-\frac{1}{4 \beta^{2}}\left(\ln \frac{1+\beta}{1-\beta}\right)^{2}\right]^{2}} .
\end{aligned}
$$

The kurtosis of a probability distribution indicates the propensity of the respective distribution to produce outliers or heavy tails.

The approximate kurtosis, denoted as $\operatorname{Kurt}^{(m, n)}\left[\Phi_{H}\right]$, can be computed by using approximations of different orders for the response's variance and fourth moment, respectively, as follows:

$$
\operatorname{Kurt}^{(m, n)}\left[\Phi_{H}\right]=\frac{\mu_{4}^{(m)}\left[\Phi_{H}\right]}{\left\{V^{(n)}\left[\Phi_{H}\right]\right\}^{2}} ; m, n=1,2, \ldots
$$

The flexible definition for the approximate skewness $\operatorname{Kurt}^{(m, n)}\left[\Phi_{H}\right]$ provided in Equation (117) aims at obtaining higher accuracy for the kurtosis with less computational effort than would be required if the fourth moment were computed at the same high order $m=n$ as the variance. Thus, the variance would be computed relatively inexpensively at an order of approximation $n>m$, while the fourth moment would be computed only up to the order of approximation $m<n$, expecting that $\operatorname{Kurt}^{(m, n)}\left[\Phi_{H}\right] \simeq \operatorname{Kurt}^{(n, n)}\left[\Phi_{H}\right]$ for a sufficiently high order $m$.

The investigation of the order of sensitivities that would be needed to achieve a target accuracy for predicting changes in the flux due to changes in the cross section $\sigma_{s}(E)$ by using the Taylor-series expansion given in Equation (16). Analogously, it is of interest to investigate the accuracy provided by the approximations $\mathbf{E}^{(n)}\left[\Phi_{H}\right], V^{(n)}\left[\Phi_{H}\right]$, $\operatorname{Skew}^{(m, n)}\left[\Phi_{H}\right]$ and $\operatorname{Kurt}^{(m, n)}\left[\Phi_{H}\right], n=1,2, \ldots ; m=1,2, \ldots$, for the expectation $\mathbf{E}\left[\Phi_{H}\right]$, variance $V\left[\Phi_{H}\right]$, skewness $S k e w\left[\Phi_{H}\right]$, and kurtosis $\operatorname{Kurt}\left[\Phi_{H}\right]$, respectively.

The expressions of the various orders of approximation for $\mathbf{E}^{(n)}\left[\Phi_{H}\right]$ and $V^{(n)}\left[\Phi_{H}\right]$, $n=1,2, \ldots$ are computed by using the expression of the approximations $\Phi_{H}^{(n)}$ in Equation (103), in order to verify independently the partial series that would be obtained by expanding (in truncated Taylor-series in $\beta$ ) the exact results obtained in Equations (97) and (101). The following results are thus obtained: 
1. If only the first-order sensitivities of $\Phi_{H}(\sigma ; E)$ with respect to $\sigma_{S}(E)$ are available, then the 1st-order approximation $\Phi_{H}^{(1)}(\sigma ; E)$ of $\Phi_{H}(\sigma ; E)$ takes on the following particular form of Equation (16):

$$
\Phi_{H}^{(1)}(\sigma ; E) \triangleq \frac{Q\left(\boldsymbol{\alpha}^{0}\right)}{E N_{H} \sigma_{m}}(1-t) ; t \triangleq \frac{\sigma_{s}(E)-\sigma_{m}}{\sigma_{m}} .
$$

The first-order approximate expectation $\mathbf{E}^{(1)}\left[\Phi_{H}\right]$ has the following expression:

$$
\mathbf{E}^{(1)}\left[\Phi_{H}\right]=\frac{Q\left(\boldsymbol{\alpha}^{0}\right)}{E N_{H} \sigma_{m}} \frac{1}{2 \beta} \int_{-\beta}^{\beta}(1-t) d t=\frac{Q\left(\boldsymbol{\alpha}^{0}\right)}{E N_{H} \sigma_{m}}=\mathbf{E}^{(0)}\left[\Phi_{H}\right] .
$$

The expectation $\mathbf{E}^{(1)}\left[\Phi_{H}\right]$ is accurate only to the zeroth-order term since the contribution from the first-order sensitivities vanishes after the integration of the respective 1st-order (odd) term over the symmetric-interval around the expectation of the parameter $\sigma_{s}(E)$.

Replacing the expression given in Equation (118) into Equation (103) yields:

$$
V^{(1)}\left[\Phi_{H}\right]=\left[\frac{Q\left(\boldsymbol{\alpha}^{0}\right)}{E N_{H} \sigma_{m}}\right]^{2}\left[\frac{1}{2 \beta} \int_{-\beta}^{\beta}(1-t)^{2} d t\right]-\left[\frac{Q\left(\boldsymbol{\alpha}^{0}\right)}{E N_{H} \sigma_{m}}\right]^{2}=\left[\frac{Q\left(\boldsymbol{\alpha}^{0}\right)}{E N_{H} \sigma_{m}}\right]^{2} \frac{\beta^{2}}{3} .
$$

The variance $V^{(1)}\left[\Phi_{H}\right]$ is accurate up to and including the contributions stemming from the 1st-order sensitivities.

2 If first-order and second-order sensitivities of $\Phi_{H}(\sigma ; E)$ with respect to $\sigma_{s}(E)$ are available, then the $2 n d$-order expansion $\Phi_{H}^{(2)}(\sigma ; E)$ of $\Phi_{H}(\sigma ; E)$ takes on the following particular form of Equation (16):

$$
\Phi_{H}^{(2)}(\sigma ; E) \triangleq \frac{Q\left(\boldsymbol{\alpha}^{0}\right)}{E N_{H} \sigma_{m}}\left(1-t+t^{2}\right) ; t \triangleq \frac{\sigma_{s}(E)-\sigma_{m}}{\sigma_{m}} .
$$

The expectation $\mathbf{E}^{(2)}\left[\Phi_{H}\right]$ has the following expression:

$$
\mathbf{E}^{(2)}\left[\Phi_{H}\right]=\frac{Q\left(\boldsymbol{\alpha}^{0}\right)}{E N_{H} \sigma_{m}} \frac{1}{2 \beta} \int_{-\beta}^{\beta}\left(1-t+t^{2}\right) d t=\frac{Q\left(\boldsymbol{\alpha}^{0}\right)}{E N_{H} \sigma_{m}}\left(1+\frac{\beta^{2}}{3}\right) .
$$

The expectation $\mathbf{E}^{(2)}\left[\Phi_{H}\right]$ is accurate up to and including the contributions stemming from the 2 nd-order sensitivities.

Replacing the expression given in Equation (121) into Equation (103) and using Equation (122) yields:

$$
\begin{gathered}
V^{(2)}\left[\Phi_{H}\right]=\left[\frac{Q\left(\boldsymbol{\alpha}^{0}\right)}{E N_{H} \boldsymbol{\sigma}_{m}}\right]^{2}\left[\frac{1}{2 \beta} \int_{-\beta}^{\beta}\left(1-t+t^{2}\right)^{2} d t\right]-\left[\frac{Q\left(\boldsymbol{\alpha}^{0}\right)}{E N_{H} \boldsymbol{\sigma}_{m}}\right]^{2}\left(1+\frac{\beta^{2}}{3}\right)^{2} \\
=\left[\frac{Q\left(\boldsymbol{\alpha}^{0}\right)}{E N_{H} \boldsymbol{\sigma}_{m}}\right]^{2}\left(\frac{\beta^{2}}{3}+\frac{4}{45} \beta^{4}\right) .
\end{gathered}
$$

The variance $V^{(2)}\left[\Phi_{H}\right]$ is accurate up to and including the contributions stemming from the 1st-order sensitivities but the contributions from the 2 nd-order sensitivities are incomplete because the term $4 \beta^{4} / 45$ is incomplete. Additional contributions to the term $4 \beta^{4} / 45$ will arise from the $3^{\text {rd }}$-order sensitivities, as will be shown below. 
3 If all sensitivities up to and including the third-order sensitivities of $\Phi_{H}(\sigma ; E)$ with respect to $\sigma_{s}(E)$ are available, then the $3 r d$-order expansion $\Phi_{H}^{(3)}(\sigma ; E)$ of $\Phi_{H}(\sigma ; E)$ takes on the following particular form of Equation (16):

$$
\Phi_{H}^{(3)}(\sigma ; E) \triangleq \frac{Q\left(\boldsymbol{\alpha}^{0}\right)}{E N_{H} \sigma_{m}}\left(1-t+t^{2}-t^{3}\right) ; t \triangleq \frac{\sigma_{s}(E)-\sigma_{m}}{\sigma_{m}} .
$$

The expectation $\mathbf{E}^{(3)}\left[\Phi_{H}\right]$ has therefore the following expression:

$$
\mathbf{E}^{(3)}\left[\Phi_{H}\right]=\frac{Q\left(\boldsymbol{\alpha}^{0}\right)}{E N_{H} \sigma_{m}} \frac{1}{2 \beta} \int_{-\beta}^{\beta}\left(1-t+t^{2}-t^{3}\right) d t=\frac{Q\left(\boldsymbol{\alpha}^{0}\right)}{E N_{H} \sigma_{m}}\left(1+\frac{\beta^{2}}{3}\right)=\mathbf{E}^{(2)}\left[\Phi_{H}\right] .
$$

The expectation $\mathbf{E}^{(3)}\left[\Phi_{H}\right]$ is only as accurate as the lower-order approximation $\mathbf{E}^{(2)}\left[\Phi_{H}\right]$, i.e., it is accurate up to and including the contributions stemming from the 2 nd-order sensitivities, because the contribution from the 3rd-order sensitivities vanishes after the integration of the respective 3 rd-order (odd) term over the symmetric-interval around the expectation of the parameter $\sigma_{s}(E)$.

Replacing the expression given in Equation (124) into Equation (103) and using Equation (125) yields:

$$
\begin{gathered}
V^{(3)}\left[\Phi_{H}\right]=\left[\frac{Q\left(\boldsymbol{\alpha}^{0}\right)}{E N_{H} \sigma_{m}}\right]^{2}\left[\frac{1}{2 \beta} \int_{-\beta}^{\beta}\left(1-t+t^{2}-t^{3}\right)^{2} d t\right]-\left[\frac{Q\left(\boldsymbol{\alpha}^{0}\right)}{E N_{H} \sigma_{m}}\right]^{2}\left(1+\frac{\beta^{2}}{3}\right)^{2} \\
=\left[\frac{Q\left(\boldsymbol{\alpha}^{0}\right)}{E N_{H} \sigma_{m}}\right]^{2}\left(\frac{\beta^{2}}{3}+\frac{22}{45} \beta^{4}+\frac{\beta^{6}}{7}\right) .
\end{gathered}
$$

4 If all sensitivities up to and including the 4th-order sensitivities of $\Phi_{H}(\sigma ; E)$ with respect to $\sigma_{s}(E)$ are available, then the 4 th-order expansion $\Phi_{H}^{(4)}(\sigma ; E)$ of $\Phi_{H}(\sigma ; E)$ takes on the following particular form of Equation (16):

$$
\Phi_{H}^{(4)}(\sigma ; E) \triangleq \frac{Q\left(\boldsymbol{\alpha}^{0}\right)}{E N_{H} \sigma_{m}}\left(1-t+t^{2}-t^{3}+t^{4}\right) ; t \triangleq \frac{\sigma_{s}(E)-\sigma_{m}}{\sigma_{m}} .
$$

The expectation $\mathbf{E}^{(4)}\left[\Phi_{H}\right]$ has the following expression:

$$
\mathbf{E}^{(4)}\left[\Phi_{H}\right]=\frac{Q\left(\alpha^{0}\right)}{E N_{H} \sigma_{m}} \frac{1}{2 \beta} \int_{-\beta}^{\beta}\left(1-t+t^{2}-t^{3}+t^{4}\right) d t=\frac{Q\left(\alpha^{0}\right)}{E N_{H} \sigma_{m}}\left(1+\frac{\beta^{2}}{3}+\frac{\beta^{4}}{5}\right) .
$$

The expectation $\mathbf{E}^{(4)}\left[\Phi_{H}\right]$ is accurate up to and including the contributions stemming from the 4 th-order sensitivities.

Replacing the expression given in Equation (128) into Equation (103) and using Equation (128) yields:

$$
\begin{gathered}
V^{(4)}\left[\Phi_{H}\right]=\left[\frac{Q\left(\alpha^{0}\right)}{E N_{H} \sigma_{m}}\right]^{2}\left[\frac{1}{2 \beta} \int_{-\beta}^{\beta}\left(1-t+t^{2}-t^{3}+t^{4}\right)^{2} d t\right]-\left[\frac{Q\left(\alpha^{0}\right)}{E N_{H} \sigma_{m}}\right]^{2}\left(1+\frac{\beta^{2}}{3}+\frac{\beta^{4}}{5}\right)^{2} \\
=\left[\frac{Q\left(\alpha^{0}\right)}{E N_{H} \sigma_{m}}\right]^{2}\left(\frac{\beta^{2}}{3}+\frac{22}{45} \beta^{4}+\frac{31}{105} \beta^{6}+\frac{16}{225} \beta^{8}\right) .
\end{gathered}
$$

The variance $V^{(4)}\left[\Phi_{H}\right]$ is accurate up to and including the contributions stemming from the 3rd-order sensitivities but the contributions from the 4 th-order sensitivities are incomplete because the term $16 \beta^{8} / 225$ is incomplete. Additional contributions to the term $16 \beta^{8} / 225$ will arise from the 5 th-order sensitivities. 
As has been mentioned in the foregoing, and as indicated by the results obtained thus far, the convergence of the infinite series-representations of the expectation and variance, $\mathbf{E}\left[\Phi_{H}\right]$ and, respectively, $V\left[\Phi_{H}\right]$ depends solely on the chosen value for the positive parameter $\beta$ : the smaller the chosen value for $\beta$, the faster the convergence of the series representation for $\mathbf{E}\left[\Phi_{H}\right]$ and for $V\left[\Phi_{H}\right]$, respectively. It is therefore of interest to investigate numerically the errors associated with the first four-orders of approximations $\mathbf{E}^{(n)}\left[\Phi_{H}\right]$ and $V^{(n)}\left[\Phi_{H}\right]$ for $n=1,2,3,4$, for several representative values for $\beta, 0<\beta<1$. Three values will be considered for the parameter $\beta$, as follows:

(a) $\quad \beta=0.2$, which corresponds to a uniform distribution of height $P\left[\sigma_{s}(E)\right]=5 / \sigma_{m}$ and (very narrow) width confined to the interval $0.8 \sigma_{m} \leq \sigma_{S}(E) \leq 1.2 \sigma_{m}$, with a standard deviation $S D\left[\sigma_{S}(E)\right]=\frac{1}{\sqrt{3}} \beta \sigma_{m}=(11.55 \%) \sigma_{m}$.

(b) $\beta=0.5$, which corresponds to a uniform distribution of height $P\left[\sigma_{s}(E)\right]=1 / \sigma_{m}$ and width confined to the interval $0.5 \sigma_{m} \leq \sigma_{\mathcal{S}}(E) \leq 1.5 \sigma_{m}$, with $S D\left[\sigma_{\mathcal{S}}(E)\right]=$ $(28.87 \%) \sigma_{m}$.

(c) $\beta=0.95$, which corresponds to a uniform distribution of height $P\left[\sigma_{s}(E)\right]=0.5263 / \sigma_{m}$ and width confined to the interval $0.05 \sigma_{m} \leq \sigma_{s}(E) \leq 1.95 \sigma_{m}$, with a standard deviation $S D\left[\sigma_{S}(E)\right]=(54.85 \%) \sigma_{m}$.

The exact normalized expectation, $\mathbf{E}_{N}\left[\Phi_{H}\right]$ and normalized variance, $V_{N}\left[\Phi_{H}\right]$ of the flux $\Phi_{H}(\sigma ; E)$ are defined by using Equations (97) and (101), as follows:

$$
\begin{gathered}
\mathbf{E}_{N}\left[\Phi_{H}\right] \triangleq \mathbf{E}\left[\Phi_{H}\right]\left[\frac{Q\left(\alpha^{0}\right)}{E N_{H}} \frac{1}{\sigma_{m}}\right]^{-1}=\frac{1}{2 \beta} \ln \frac{1+\beta}{1-\beta} \\
V_{N}\left[\Phi_{H}\right] \triangleq V\left[\Phi_{H}\right]\left[\frac{Q\left(\alpha^{0}\right)}{E N_{H} \sigma_{m}}\right]^{-2}=\left[\frac{1}{1-\beta^{2}}-\frac{1}{4 \beta^{2}}\left(\ln \frac{1+\beta}{1-\beta}\right)^{2}\right] .
\end{gathered}
$$

The normalized exact standard deviation is defined as follows: $S D_{N}\left[\Phi_{H}\right]=\sqrt{V_{N}\left[\Phi_{H}\right]}$. The normalized $n^{\text {th }}$-order approximations $\mathbf{E}_{N}^{(n)}\left[\Phi_{H}\right], V_{N}^{(n)}\left[\Phi_{H}\right]$ and $S D_{N}^{(n)}\left[\Phi_{H}\right]$ of the expectation, variance and standard deviation of the flux response $\Phi_{H}(\sigma ; E)$, respectively, are defined as follows:

$$
\begin{aligned}
& \mathbf{E}_{N}^{(n)}\left[\Phi_{H}\right] \triangleq \mathbf{E}^{(n)}\left[\Phi_{H}\right]\left[\frac{Q\left(\boldsymbol{\alpha}^{0}\right)}{E N_{H}} \frac{1}{\sigma_{m}}\right]^{-1} ; V_{N}^{(n)}\left[\Phi_{H}\right] \triangleq V^{(n)}\left[\Phi_{H}\right]\left[\frac{Q\left(\boldsymbol{\alpha}^{0}\right)}{E N_{H} \sigma_{m}}\right]^{-2} ; \\
& S D_{N}^{(n)}\left[\Phi_{H}\right] \triangleq \sqrt{V_{N}^{(n)}\left[\Phi_{H}\right]} ; n=1,2,3,4 .
\end{aligned}
$$

The results obtained from using the exact expression in provided in Equations (130) and (131) are compared to the approximate results obtained by using the expressions provided in Equation (132), for $n=1,2,3,4$, and for $\beta=0.2, \beta=0.5$ and $\beta=0.95$, respectively. Since all four moments (i.e., expectation, variance, skewness and kurtosis) of the distribution of the response $\Phi_{H}(\sigma ; E)$ are even functions of $\beta$, it suffices to consider only positive values for $\beta$. The results obtained for the expectation and variance of the predicted distribution of the response $\Phi_{H}(\sigma ; E)$ are summarized in Tables $1-3$, below.

The following conclusions can be drawn from the results presented in Tables 1-3:

For very small values $(\beta=0.2)$ of the parameter $\beta$, the expansions (in powers of $\beta$ ), which represent the various orders $(n=1,2,3,4)$ of approximations of the normalized expectation $\mathbf{E}_{N}^{(n)}\left[\Phi_{H}\right]$ and normalized variance $V_{N}^{(n)}\left[\Phi_{H}\right]$ of the unknown distribution of the flux $\Phi_{H}(\sigma ; E)$ converge very quickly to the respective exact values $\mathbf{E}_{N}\left[\Phi_{H}\right]$ and $V_{N}\left[\Phi_{H}\right]$, as indicated by the results presented in Table 3. Already the 1st-order approximations $\mathbf{E}_{N}^{(1)}\left[\Phi_{H}\right]$ and $V_{N}^{(1)}\left[\Phi_{H}\right]$ yield results that are within $5 \%$ of the exact values of the expectation and, respectively, variance of the flux $\Phi_{H}(\sigma ; E)$. The 3rd-order approximation $\mathbf{E}_{N}^{(1)}\left[\Phi_{H}\right]$ is within $0.07 \%$ of the exact value for the expectation of the flux $\Phi_{H}(\sigma ; E)$ of the while the 3rd-order approximation $V_{N}^{(3)}\left[\Phi_{H}\right]$ of the standard deviation of the flux $\Phi_{H}(\sigma ; E)$ is 
exact up to at least four decimals. Recall that a very small value of $\beta$ implies that the measurement $\sigma_{m} \triangleq \sigma_{s}\left(E_{m}\right)$ is extremely accurate, i.e., the uniform distribution of $\sigma_{s}\left(E_{m}\right)$ around $\sigma_{m}$ is extremely narrow.

Table 1. Exact and approximate values for the expectation, variance and standard deviation of $\Phi_{H}(\sigma ; E)$ for $\beta=0.2$.

\begin{tabular}{|c|c|c|c|c|c|}
\hline Moment & Exact Value & $\begin{array}{l}\text { 1st-Order Value } \\
\text { (Error }^{1} \text { ) }\end{array}$ & $\begin{array}{l}\text { 2nd-Order Value } \\
\left(\text { Error }^{1}\right)\end{array}$ & $\begin{array}{l}\text { 3rd-Order Value } \\
\text { (Error }^{1} \text { ) }\end{array}$ & $\begin{array}{l}\text { 4th-Order Value } \\
\text { (Error }^{1} \text { ) }\end{array}$ \\
\hline $\mathbf{E}_{N}\left[\Phi_{H}\right]$ & 1.014 & $\begin{array}{l}1.000 \\
(1.4 \%)\end{array}$ & $\begin{array}{l}1.000 \\
(1.4 \%)\end{array}$ & $\begin{array}{c}1.013 \\
(0.07 \%)\end{array}$ & $\begin{array}{c}1.013 \\
(0.07 \%)\end{array}$ \\
\hline$V_{N}\left[\Phi_{H}\right]$ & 0.0142 & $\begin{array}{c}0.0133 \\
(5 \%)\end{array}$ & $\begin{array}{c}0.0134 \\
(3.88 \%)\end{array}$ & $\begin{array}{c}0.0142 \\
\left(\text { exact }^{2}\right)\end{array}$ & $\begin{array}{c}0.0142 \\
\left(\text { exact }^{2}\right)\end{array}$ \\
\hline$S D_{N}\left[\Phi_{H}\right]$ & 0.1190 & $\begin{array}{c}0.1155 \\
(3 \%)\end{array}$ & $\begin{array}{l}0.1158 \\
(2.7 \%)\end{array}$ & $\begin{array}{c}0.0190 \\
\left(\text { exact }^{2}\right)\end{array}$ & $\begin{array}{c}0.0190 \\
\left(\text { exact }^{2}\right)\end{array}$ \\
\hline
\end{tabular}

${ }^{1}$ Error $=($ exact-approximate $) /$ exact. ${ }^{2}$ Exact to at least four decimal digits.

Table 2. Exact and approximate values for the expectation, variance and standard deviation of $\Phi_{H}(\sigma ; E)$ for $\beta=0.5$.

\begin{tabular}{|c|c|c|c|c|c|}
\hline Moment & Exact Value & $\begin{array}{l}\text { 1st-Order Value } \\
\quad\left(\text { Error }^{1}\right)\end{array}$ & $\begin{array}{l}\text { 2nd-Order Value } \\
\text { (Error }^{1} \text { ) }\end{array}$ & $\begin{array}{l}\text { 3rd-Order Value } \\
\text { (Error }^{\mathbf{1}} \text { ) }\end{array}$ & $\begin{array}{c}\text { 4th-Order Value } \\
\text { (Error }{ }^{1} \text { ) }\end{array}$ \\
\hline $\mathbf{E}_{N}\left[\Phi_{H}\right]$ & 1.0990 & $\begin{array}{c}1.000 \\
(9.01 \%)\end{array}$ & $\begin{array}{c}1.083 \\
(1.43 \%)\end{array}$ & $\begin{array}{c}1.083 \\
(1.43 \%)\end{array}$ & $\begin{array}{c}1.096 \\
(0.27 \%)\end{array}$ \\
\hline$V_{N}\left[\Phi_{H}\right]$ & 0.1264 & $\begin{array}{c}0.0833 \\
(34.05 \%)\end{array}$ & $\begin{array}{c}0.0889 \\
(29.67 \%)\end{array}$ & $\begin{array}{c}0.1161 \\
(8.13 \%)\end{array}$ & $\begin{array}{c}0.1188 \\
(6.03 \%)\end{array}$ \\
\hline$S D_{N}\left[\Phi_{H}\right]$ & 0.3555 & $\begin{array}{c}0.2887 \\
(18.8 \%)\end{array}$ & $\begin{array}{c}0.2981 \\
(16.2 \%)\end{array}$ & $\begin{array}{c}0.3375 \\
(5.06 \%)\end{array}$ & $\begin{array}{c}0.3447 \\
(3.05 \%)\end{array}$ \\
\hline
\end{tabular}

${ }^{1}$ Error $=$ (exact-approximate) $/$ exact.

Table 3. Exact and approximate values for the expectation, variance and standard deviation of $\Phi_{H}(\sigma ; E)$ for $\beta=0.95$.

\begin{tabular}{|c|c|c|c|c|c|}
\hline Moment & Exact Value & $\begin{array}{l}\text { 1st-Order Value } \\
\quad\left(\text { Error }^{1}\right)\end{array}$ & $\begin{array}{l}\text { 2nd-Order Value } \\
\text { (Error }{ }^{1} \text { ) }\end{array}$ & $\begin{array}{l}\text { 3rd-Order Value } \\
\quad \text { (Error }^{1} \text { ) }\end{array}$ & $\begin{array}{l}\text { 4th-Order Value } \\
\text { (Error }{ }^{1} \text { ) }\end{array}$ \\
\hline $\mathbf{E}_{N}\left[\Phi_{H}\right]$ & 1.928 & $\begin{array}{c}1.000 \\
(48.14 \%)\end{array}$ & $\begin{array}{c}1.301 \\
(32.53 \%)\end{array}$ & $\begin{array}{c}1.301 \\
(32.53 \%)\end{array}$ & $\begin{array}{c}1.464 \\
(24.1 \%)\end{array}$ \\
\hline$V_{N}\left[\Phi_{H}\right]$ & 6.5385 & $\begin{array}{c}0.3008 \\
(95.40 \%)\end{array}$ & $\begin{array}{c}0.3732 \\
(94.29 \%)\end{array}$ & $\begin{array}{c}0.8040 \\
(87.70 \%)\end{array}$ & $\begin{array}{c}0.9632 \\
(85.27 \%)\end{array}$ \\
\hline$S D_{N}\left[\Phi_{H}\right]$ & 2.5570 & $\begin{array}{c}0.5485 \\
(78.55 \%)\end{array}$ & $\begin{array}{c}0.6109 \\
(76.11 \% \%)\end{array}$ & $\begin{array}{c}0.8967 \\
(64.93 \%)\end{array}$ & $\begin{array}{c}0.9814 \\
(62.62 \%)\end{array}$ \\
\hline
\end{tabular}

${ }^{1}$ Error $=($ exact-approximate $) /$ exact.

The value $\beta=0.5$ is characteristic of a measurement of moderate precision, so it is representative of the majority of evaluated cross sections such as the scattering cross section $\sigma_{s}\left(E_{m}\right)$. The results in Table 4 indicate that the 1st-order approximations are not satisfactory: the first-order approximation $\mathbf{E}_{N}^{(1)}\left[\Phi_{H}\right]$ of the exact expectation $\mathbf{E}_{N}\left[\Phi_{H}\right]$ is in error by $9 \%$, while the first-order approximation $S D_{N}^{(1)}\left[\Phi_{H}\right]$ of the exact standard deviation $S D_{N}\left[\Phi_{H}\right]$ is in error by $19 \%$. Only the 3rd-order (or higher-order) approximations, $\mathbf{E}_{N}^{(3)}\left[\Phi_{H}\right]$ and $S D_{N}^{(3)}\left[\Phi_{H}\right]$, would provide values for the expectation and, respectively, standard deviation of the flux $\Phi_{H}(\sigma ; E)$ which would be within $5 \%$ of the respective exact values. 
Table 4. Exact and approximate values for the third-order moment and skewness of $\Phi_{H}(\sigma ; E)$.

\begin{tabular}{|c|c|c|c|}
\hline 3rd-Order Moment & $\beta=0.2$ & $\beta=0.5$ & $\beta=0.95$ \\
\hline Exact: $\mu_{3}\left[\Phi_{H}\right]$ & 0.0005 & 0.0353 & 60.20 \\
\hline Exact: Skew $\left[\Phi_{H}\right]$ & 0.2955 & 0.7857 & 3.6 \\
\hline 1st-order: $\mu_{3}^{(1)}\left[\Phi_{H}\right]\left(\right.$ Error $\left.^{1}\right)$ & $\begin{array}{c}0.0000 \\
(100 \%)\end{array}$ & $\begin{array}{r}0.0000 \\
(100 \%)\end{array}$ & $\begin{array}{l}0.0000 \\
(100 \%)\end{array}$ \\
\hline 1st-order: Skew ${ }^{(1,1)}\left[\Phi_{H}\right]\left(\right.$ Error $\left.^{1}\right)$ & $\begin{array}{r}0.0000 \\
(100 \%)\end{array}$ & $\begin{array}{c}0.0000 \\
(100 \%)\end{array}$ & $\begin{array}{c}0.0000 \\
(100 \%)\end{array}$ \\
\hline 2nd-order: $\mu_{3}^{(2)}\left[\Phi_{H}\right]\left(\right.$ Error $\left.^{1}\right)$ & $\begin{array}{l}0.0004 \\
(20 \%)\end{array}$ & $\begin{array}{c}0.0267 \\
(32.2 \%)\end{array}$ & $\begin{array}{c}0.2172 \\
(99.6 \%)\end{array}$ \\
\hline $\begin{array}{l}\text { 2nd-order: } \operatorname{Skew}^{(2,2)}\left[\Phi_{H}\right] \\
\left(\text { Error }^{1}\right)\end{array}$ & $\begin{array}{c}0.2576 \\
(13.2 \%)\end{array}$ & $\begin{array}{c}1.0073 \\
(-28.3 \%)\end{array}$ & $\begin{array}{l}0.9527 \\
(73.6 \%)\end{array}$ \\
\hline $\begin{array}{l}\operatorname{Skew}^{(2,4)}\left[\Phi_{H}\right] \\
\quad\left(\text { Error }^{1}\right)\end{array}$ & $\begin{array}{l}0.2364 \\
(20 \%)\end{array}$ & $\begin{array}{c}0.6521 \\
(17.0 \%)\end{array}$ & $\begin{array}{c}0.2298 \\
(93.6 \%)\end{array}$ \\
\hline
\end{tabular}

${ }^{1}$ Error $=($ exact-approximate $) /$ exact.

The value $\beta=0.95$ characterizes either an imprecise measurement or the need to construct "tolerance intervals" that cover a large segment of the unknown distribution of the quantity under investigation, i.e., the flux $\Phi_{H}(\sigma ; E)$, in this illustrative example. As the results in Table 5 indicate, the convergence of the various approximations is extremely slow. Even the 4th-order approximations $\mathbf{E}_{N}^{(4)}\left[\Phi_{H}\right]$ and $S D_{N}^{(4)}\left[\Phi_{H}\right]$ are very far off the exact values $\mathbf{E}_{N}\left[\Phi_{H}\right]$ and $S D_{N}\left[\Phi_{H}\right]$, respectively, of the expectation and the standard deviation of $\Phi_{H}(\sigma ; E)$.

Table 5. Exact and approximate values for the fourth-order moment of $\Phi_{H}(\sigma ; E)$.

\begin{tabular}{|c|c|c|c|}
\hline 4th-Order Moment & $\beta=0.2$ & $\beta=0.5$ & $\beta=0.95$ \\
\hline Exact: $\mu_{4}\left[\Phi_{H}\right]$ & 0.0004 & 0.0411 & 779.47 \\
\hline Exact: $\operatorname{Kurt}\left[\Phi_{H}\right]$ & 1.9837 & 2.5725 & 18.23 \\
\hline $\begin{array}{l}\text { 1st-order: } \mu_{4}^{(1)}\left[\Phi_{H}\right] \\
\quad\left(\text { Error }^{1}\right)\end{array}$ & $\begin{array}{l}0.0003 \\
(25 \%)\end{array}$ & $\begin{array}{c}0.0125 \\
(69.6 \%)\end{array}$ & $\begin{array}{l}0.1629 \\
(100 \%)\end{array}$ \\
\hline $\begin{array}{c}\text { 1st-order: } \operatorname{Kurt}^{(1,1)}\left[\Phi_{H}\right] \\
\left(\text { Error }^{1}\right)\end{array}$ & $\begin{array}{c}1.6960 \\
(14.5 \%)\end{array}$ & $\begin{array}{c}1.8014 \\
(29.97 \%)\end{array}$ & $\begin{array}{l}1.8004 \\
(90 \%)\end{array}$ \\
\hline $\begin{array}{l}\text { 2nd-order: } \mu_{4}^{(2)}\left[\Phi_{H}\right] \\
\quad\left(\text { Error }^{1}\right)\end{array}$ & $\begin{array}{c}0.0004 \\
\left(\text { Exact }^{2}\right)\end{array}$ & $\begin{array}{l}0.0259 \\
(37 \%)\end{array}$ & $\begin{array}{c}0.7930 \\
(99.99 \%)\end{array}$ \\
\hline $\begin{array}{c}\text { 2nd-order: } \operatorname{Kurt}^{(2,2)}\left[\Phi_{H}\right] \\
\left(\text { Error }^{1}\right)\end{array}$ & $\begin{array}{c}2.2277 \\
(-12.3 \%)\end{array}$ & $\begin{array}{c}3.2771 \\
(-27.39)\end{array}$ & $\begin{array}{c}5.694 \\
(68.8 \%)\end{array}$ \\
\hline $\begin{array}{l}\operatorname{Kurt}^{(2,4)}\left[\Phi_{H}\right] \\
\quad\left(\text { Error }^{1}\right)\end{array}$ & $\begin{array}{c}1.9837 \\
\left(\text { Exact }^{2}\right)\end{array}$ & $\begin{array}{l}1.8351 \\
(28.6 \%)\end{array}$ & $\begin{array}{c}0.8548 \\
(95.3 \%)\end{array}$ \\
\hline
\end{tabular}

${ }^{1}$ Error $=$ (exact-approximate) $/$ exact. ${ }^{2}$ Exact to at least four decimal digits.

The accuracies of the 1st-order and 2nd-order approximations for the third-order moment, $\mu_{3}\left[\Phi_{H}\right]$, and the skewness, $\operatorname{Skew}\left[\Phi_{H}\right]$, of $\Phi_{H}(\sigma ; E)$ are illustrated by the results presented in Table 5. The exact moment, $\mu_{3}\left[\Phi_{H}\right]$, of the predicted distribution of the response $\Phi_{H}(\sigma ; E)$ is positive, indicating a distribution which would be skewed toward values more positive than the distribution's expected value $\sigma_{m}$. As the positive values of the parameter $\beta$ increase towards unity, which would represent an increasingly broader distribution for $\Phi_{H}(\sigma ; E), \mu_{3}\left[\Phi_{H}\right]$ also increases; hence, the skewness of the distribution for $\Phi_{H}(\sigma ; E)$ also increases. With pronounced skewness, standard statistical inference procedures such as a confidence interval for a mean will be not only incorrect, in the sense that the true coverage level will differ from the nominal (e.g., 95\%) level, but they will also result in unequal error probabilities on each side.

Very importantly, the results presented in bold in Table 4 indicate that the first-order approximation for the third-moment $\mu_{3}^{(1)}\left[\Phi_{H}\right]$ of the predicted response distribution is always zero. Thus, using just the first-order response sensitivities will always be $100 \%$ incorrect unless the distribution of the response under consideration happens to be symmetric. Conversely, if only first-order sensitivities are considered, the third-order moment 
of the response is always zero. Thus, the response distribution is always predicted to be symmetrical if only first-order sensitivities are considered. Hence, a "first-order sensitivity and uncertainty quantification" will always produce an erroneous third moment (and hence skewness) of the predicted response distribution, unless the distribution happens to be symmetrical. At least second-order sensitivities must be used in order to estimate the third-order moment (and hence the skewness) of the response distribution.

The overall conclusion that follows from the results presented in Table 4 is that loworder (i.e., 1st- and 2nd-order) approximations for the third-order moment, $\mu_{3}\left[\Phi_{H}\right]$, of the response distribution cannot provide reliably correct values for the skewness. Significantly higher-order approximations, which would require the computation of high-order response sensitivities to model parameters, are needed in order to obtain reliable values for the third-order response moment $\mu_{3}\left[\Phi_{H}\right]$ and, hence, for the skewness of the response distribution. Comparing the results in Table 4 for increasing (positive) values for the parameter $\beta$, the low-order (1st-and 2nd-order) approximations become increasingly unreliable as $\beta$ increases toward unity. This trend indicates that, as the response distribution becomes wider, sensitivities of increasingly higher-order would be needed in order to improve the predicted values for the response skewness.

The accuracies of the 1st-order and 2nd-order approximations for the fourth-order moment $\mu_{4}\left[\Phi_{H}\right]$ of $\Phi_{H}(\sigma ; E)$ are illustrated by the results presented in Table 5 . The results presented in Table 5 also indicate that for small values of the parameter $\beta$, the first-order approximation, $\mu_{4}^{(1)}\left[\Phi_{H}\right]$, provides an approximate value which differs by $25 \%$ from the exact value $\mu_{4}\left[\Phi_{H}\right]$, while the second-order approximation $\mu_{4}^{(2)}\left[\Phi_{H}\right]$ provides the exact first four significant digits of the exact value of $\mu_{4}\left[\Phi_{H}\right]$. The main conclusions that follow from the results presented in Table 5 are similar to those that were drawn from the results presented in Table 4 for the response's third-order moment and skewness, namely: low-order (i.e., 1st- and 2nd-order) approximations for the fourth-order moment, $\mu_{4}\left[\Phi_{H}\right]$, of the response distribution can provide the correct sign of the response kurtosis, but cannot provide reliably correct values for the kurtosis. Significantly higher-order approximations, which would require the computation of high-order response sensitivities to model parameters, are needed in order to obtain reliable values for the fourth-order response moment $\mu_{4}\left[\Phi_{H}\right]$ and, hence, for the kurtosis of the response distribution. The results in Table 5 indicate that the low-order (1st-and 2 nd-order) approximations become increasingly unreliable as $\beta$ increases (with positive values) toward unity, which means that, as the response distribution becomes wider, sensitivities of increasingly higherorder would be needed in order to improve the predicted values for the response kurtosis. If accurate values for the response kurtosis are required, it is not possible to circumvent the need for computing high-order sensitivities.

\section{The Third-Order Comprehensive Adjoint Sensitivity Analysis Methodology for Nonlinear Systems (3rd-CASAM-N)}

The mathematical framework underlying the Third-Order Comprehensive Adjoint Sensitivity Analysis Methodology for Nonlinear Systems (3rd-CASAM-N) uses, as the starting point, the mathematical framework of the first- and second-order comprehensive adjoint sensitivity analysis methodologies, which have been presented in [24,25]. However, the novel 3rd-CASAM-N methodology cannot be comprehended without understating the 1st-CASAM-N and 2nd-CASAM-N. For convenient reference, therefore, the mathematical frameworks of the 1st-CASAM-N and 2nd-CASAM-N are summarized in the Appendix A. It is recommended that the derivations summarized in the Appendix A be perused prior to proceeding with the new material presented below.

The 3rd-order G-differential of the model response $R(\mathbf{e})$ to variations $\mathbf{h} \triangleq(\delta \boldsymbol{\alpha}, \delta \mathbf{u})$ in the model parameters and state functions around $\mathbf{e}^{0}=\left(\boldsymbol{\alpha}^{0}, \mathbf{u}^{0}\right)$ provides the 3rd-order total sensitivity of $R(\mathbf{e})$ and will exist if the partial 2 nd-order sensitivities $R_{i_{1} i_{2}}^{(2)}\left(\alpha^{0}, \varphi^{(2,0)} ; \psi_{1, i_{1}}^{(2,0)}, \Psi_{2, i_{1}}^{(2,0)}\right)$ satisfy the conditions represented by Equations (A2) and (A3), presented in the Appendix A. 
The 3rd-order G-differential of $R(\mathbf{e})$ will be denoted as $\delta^{(3)} R\left(\mathbf{e}^{0} ; \mathbf{h}\right)$, and is defined inductively as "the G-differential of the 2nd-order G-differential," i.e.,

$$
\begin{aligned}
\delta^{(3)} R\left(\mathbf{e}^{0} ; \mathbf{h}_{1}, \mathbf{h}_{2}, \mathbf{h}_{3}\right) & \triangleq\left\{\frac{d}{d \varepsilon}\left[\delta^{(2)} R\left(\mathbf{e}^{0}+\varepsilon \mathbf{h}_{3} ; \mathbf{h}_{1}, \mathbf{h}_{2}\right)\right]\right\}_{\varepsilon=0} \\
& =\left\{\frac{\partial^{2}}{\partial \varepsilon_{1} \partial \varepsilon_{2} \partial \varepsilon_{3}}\left[R\left(\mathbf{e}^{0}+\varepsilon_{1} \mathbf{h}_{1}+\varepsilon_{2} \mathbf{h}_{2}+\varepsilon_{3} \mathbf{h}_{3}\right)\right]\right\}_{\varepsilon_{1}=\varepsilon_{2}=\varepsilon_{3}=0^{\prime}}
\end{aligned}
$$

for $\varepsilon_{1}, \varepsilon_{2}, \varepsilon_{3} \in \mathrm{F}$, and all (i.e., arbitrary) vectors of variations $\mathbf{h}_{i} \in \mathrm{E}=\mathrm{E}_{\alpha} \oplus \mathrm{E}_{u} ; i=$ $1,2,3$; around $\mathbf{e}^{0}=\left(\boldsymbol{\alpha}^{0}, \mathbf{u}^{0}\right) \in \mathrm{E}$. The total 3rd-order response differential $\delta^{(3)} R\left(\mathbf{e}^{0} ; \mathbf{h}\right)$ is trilinear in the parameter variations $\delta \alpha_{i}$, a fact that will be denoted as $D^{(3)} R\left(\mathbf{e}^{0} ; \mathbf{h}_{1}, \mathbf{h}_{2}\right)$, so that Equation (133) will ultimately be expressed in the form $D^{(3)} R\left(\mathbf{e}^{0} ; \mathbf{h}_{1}, \mathbf{h}_{2}, \mathbf{h}_{3}\right)=$ $\sum_{i_{3}, i_{2}, i_{1}=1}^{N_{\alpha}} R_{i_{3} i_{2} i_{1}}^{(3)} \delta \alpha_{i_{3}} \delta \alpha_{i_{2}} \delta \alpha_{i_{1}}$, where the quantities $R_{i_{3} i_{2} i_{1}}^{(3)}$ do not depend on the parameter variations and represent the mixed 2nd-order partial sensitivities (G-derivatives) of the model response $R(\mathbf{e})$ at $\mathbf{e}^{0}$. The exact and efficient computation of the 3rd-order partial sensitivities (G-derivatives) $R_{i_{3} i_{2} i_{1}}^{(3)}$ will be the objective of the developments to be presented in the remainder of this sub-section.

As indicated in Equation (A56) in the Appendix A, the 2nd-order partial sensitivities are functionals of the form $R_{i_{1} i_{2}}^{(2)}\left(\alpha, \varphi^{(2)} ; \psi_{i_{1}}^{(2)}\right) \in \mathrm{H}_{\alpha} \oplus 2 \otimes \mathrm{H}_{\psi}^{(2)}\left(\Omega_{x}\right) \rightarrow$ F. In order to simplify the notation to follow, an arbitrary point in the product-Hilbert-space $2 \otimes \mathrm{H}_{\psi}^{(2)}\left(\Omega_{x}\right)$ will be denoted as $\boldsymbol{\varphi}_{i_{1}}^{(3)} \triangleq\left(\boldsymbol{\varphi}^{(2)} ; \boldsymbol{\psi}_{i_{1}}^{(2)}\right) \in 2 \otimes \mathrm{H}_{\psi}^{(2)}\left(\Omega_{x}\right)$, which is a short-hand notation for the equivalent notation $\boldsymbol{\varphi}_{i_{1}}^{(3)}(\mathbf{x}) \triangleq\left(\mathbf{u} ; \boldsymbol{\psi}^{(1)} ; \boldsymbol{\psi}_{1, i_{1}}^{(2)} \boldsymbol{\psi}_{i, i_{1}}^{(2)}\right)=\mathrm{H}_{u}\left(\Omega_{x}\right) \oplus \mathrm{H}_{\psi}^{(1)}\left(\Omega_{x}\right) \oplus$ $\mathrm{H}_{\psi}^{(2)}\left(\Omega_{x}\right)$. A "nominal value" of $\boldsymbol{\varphi}_{i_{1}}^{(3)}$ will be denoted as $\boldsymbol{\varphi}_{i_{1}}^{(3,0)} \triangleq\left(\boldsymbol{\varphi}^{(2,0)} ; \boldsymbol{\psi}_{i_{1}}^{(2,0)}\right) \in$ $2 \otimes \mathrm{H}_{\psi}^{(2)}\left(\Omega_{x}\right)$. A vector of arbitrary variations around $\varphi^{(3,0)}$ will be denoted as $\delta \varphi^{(3)} \triangleq$ $\left(\delta \mathbf{u}, \delta \boldsymbol{\psi}^{(1)}, \delta \boldsymbol{\psi}_{1, i_{1}}^{(2)} \delta \boldsymbol{\psi}_{2, i_{1}}^{(2)}\right)$. Occasionally, however, the respective arguments of $\boldsymbol{\varphi}^{(3)}(\mathbf{x})$, $\boldsymbol{\varphi}^{(3,0)}(\mathbf{x})$ and $\delta \varphi^{(3)}(\mathbf{x})$ may be written out explicitly, whenever needed for clarity.

The definition given in Equation (133) implies that

$\delta^{(3)} R\left(\mathbf{e}^{0} ; \mathbf{h}_{1}, \mathbf{h}_{2}, \mathbf{h}_{3}\right)=\sum_{i_{1}=1}^{T P} \sum_{i_{2}=1}^{T P} \delta R_{i_{1} i_{2}}^{(2)}\left(\boldsymbol{\alpha}^{0}, \boldsymbol{\varphi}_{i_{1}}^{(3,0)} ; \delta \boldsymbol{\alpha}, \delta \mathbf{u}, \delta \boldsymbol{\psi}^{(1)}, \delta \boldsymbol{\psi}_{1, i_{1}}^{(2)}, \delta \boldsymbol{\psi}_{2, i_{1}}^{(2)}\right) \delta \alpha_{i_{1}} \delta \alpha_{i_{2}}$,

where

$$
\begin{aligned}
& \delta R_{i_{1} i_{2}}^{(2)}\left(\boldsymbol{\alpha}^{0}, \boldsymbol{\varphi}^{(3,0)} ; \delta \boldsymbol{\alpha}, \delta \mathbf{u}, \delta \boldsymbol{\psi}^{(1)}, \delta \boldsymbol{\psi}_{1, i_{1}}^{(2)}, \delta \boldsymbol{\Psi}_{i, i_{1}}^{(2)}\right) \triangleq \\
& \left\{\frac{d}{d \varepsilon} R_{i_{1} i_{2}}^{(2)}\left(\boldsymbol{\alpha}^{0}+\varepsilon \delta \boldsymbol{\alpha}, \mathbf{u}^{0}+\varepsilon \delta \mathbf{u} ; \boldsymbol{\psi}^{(1,0)}+\varepsilon \delta \boldsymbol{\psi}^{(1)} ; \boldsymbol{\psi}_{1, i_{1}}^{(2,0)}+\varepsilon \delta \boldsymbol{\psi}_{1, i_{1},}^{(2)} \boldsymbol{\Psi}_{2, i_{1}}^{(2,0)}+\varepsilon \delta \boldsymbol{\psi}_{2, i_{1}}^{(2)}\right)\right\}_{\varepsilon=0} \\
& =\left(\delta R_{i_{1} i_{2}}^{(2)}\right)_{\text {direct }}+\left(\delta R_{i_{1} i_{2}}^{(2)}\right)_{\text {indirect }^{\prime}}
\end{aligned}
$$

with

$$
\left(\delta R_{i_{1} i_{2}}^{(2)}\right)_{\text {direct }} \triangleq\left\{\frac{\partial R_{i_{1} i_{2}}^{(2)} \delta \alpha}{\partial \boldsymbol{\alpha}}\right\}_{\left(\boldsymbol{\alpha}^{0}, \boldsymbol{\varphi}_{i_{1}}^{(3,0)}\right)},
$$

and

$$
\left(\delta R_{i_{1} i_{2}}^{(2)}\right)_{\text {indirect }} \triangleq\left\{\frac{\partial R_{i_{1} i_{2}}^{(2)}}{\partial \mathbf{u}} \delta \mathbf{u}+\frac{\partial R_{i_{1} i_{2}}^{(2)}}{\partial \boldsymbol{\psi}^{(1)}} \delta \boldsymbol{\psi}^{(1)}+\frac{\partial R_{i_{1} i_{2}}^{(2)}}{\partial \boldsymbol{\psi}_{1, i_{1}}^{(2)}} \delta \boldsymbol{\psi}_{1, i_{1}}^{(2)}+\frac{\partial R_{i_{1} i_{2}}^{(2)}}{\partial \boldsymbol{\psi}_{2, i_{1}}^{(2)}} \delta \boldsymbol{\psi}_{2, i_{1}}^{(2)}\right\}_{\left(\boldsymbol{\alpha}^{0}, \boldsymbol{\varphi}_{i_{1}}^{(3,0)}\right)}
$$

The direct effect term, $\left(\delta R_{i_{1} i_{2}}^{(2)}\right)_{\text {direct }}$, can be computed immediately at this stage while the "indirect effect term," $\left(\delta R_{i_{1} i_{2}}^{(2)}\right)_{\text {indirect }}$ can be computed only after determin- 
ing the vectors of variations $\delta \mathbf{u}, \delta \boldsymbol{\psi}^{(1)}, \delta \boldsymbol{\psi}_{1, i_{1}}^{(2)}$, and $\delta \boldsymbol{\psi}_{i, i_{1}}^{(2)}$, which are related to the parameter variations $\delta \alpha$. Two relations between all of these variations are obtained by taking the G-differential of the 2nd-LASS, see Equations (A54), (A55) and (A43) in the Appendix $A$, at the phase-space point $\left(\alpha^{0}, \mathbf{u}^{0} ; \boldsymbol{\psi}^{(1,0)} ; \boldsymbol{\psi}_{1, i_{1}}^{(2,0)}, \boldsymbol{\psi}_{2, i_{1}}^{(2,0)}\right)$ in the neighborhood of $\left(\alpha^{0}+\varepsilon \delta \alpha, \mathbf{u}^{0}+\varepsilon \delta \mathbf{u} ; \boldsymbol{\psi}^{(1,0)}+\varepsilon \delta \boldsymbol{\psi}^{(1)} ; \boldsymbol{\psi}_{1, i_{1}}^{(2,0)}+\varepsilon \delta \psi_{1, i_{1}}^{(2)}, \boldsymbol{\psi}_{2, i_{1}}^{(2,0)}+\varepsilon \delta \boldsymbol{\psi}_{2, i_{1}}^{(2)}\right)$, to obtain the following equations:

$$
\begin{aligned}
& \mathbf{F}_{11}^{(3)}\left(\boldsymbol{\alpha}^{0}, \mathbf{u}^{0}\right) \delta \boldsymbol{\psi}_{2, i_{1}}^{(2)}+\mathbf{F}_{12}^{(3)}\left(\boldsymbol{\alpha}^{0}, \mathbf{u}^{0}\right) \delta \boldsymbol{\psi}_{1, i_{1}}^{(2)}+\mathbf{F}_{13}^{(3)}\left(\boldsymbol{\alpha}^{0}, \mathbf{u}^{0} ; \boldsymbol{\psi}^{(1,0)}\right) \delta \boldsymbol{\psi}^{(1)}+\mathbf{F}_{14}^{(3)}\left(\boldsymbol{\alpha}^{0}, \mathbf{u}^{0} ; \boldsymbol{\psi}^{(1,0)}\right) \delta \mathbf{u} \\
& =\mathbf{Q}_{1, i_{1}}^{(3)}\left(\boldsymbol{\alpha}^{0}, \boldsymbol{\varphi}_{i_{1}}^{(3,0)} ; \delta \boldsymbol{\alpha}\right), \quad \mathbf{x} \in \Omega_{x,} i_{1}=1, \ldots, T P, \\
& \mathbf{F}_{22}^{(3)}\left(\boldsymbol{\alpha}^{0}, \mathbf{u}^{0}\right) \delta \boldsymbol{\psi}_{1, i_{1}}^{(2)}+\mathbf{F}_{23}^{(3)}\left(\boldsymbol{\alpha}^{0}, \mathbf{u}^{0} ; \boldsymbol{\psi}^{(1,0)}\right) \delta \boldsymbol{\psi}^{(1)}+\mathbf{F}_{24}^{(3)}\left(\boldsymbol{\alpha}^{0}, \mathbf{u}^{0}\right) \delta \mathbf{u} \\
& =\mathbf{Q}_{2, i_{1}}^{(3)}\left(\boldsymbol{\alpha}^{0}, \mathbf{u}^{0} ; \boldsymbol{\psi}^{(1,0)} ; \boldsymbol{\psi}_{1, i_{1}}^{(2,0)} ; \delta \boldsymbol{\alpha}\right), \quad \mathbf{x} \in \Omega_{x}, i_{1}=1, \ldots, T P,
\end{aligned}
$$

together with the following variational boundary conditions

$$
\delta \mathbf{B}_{A}^{(2)}\left(\boldsymbol{\alpha}^{0}, \boldsymbol{\varphi}_{i_{1}}^{(3,0)} ; \delta \boldsymbol{\alpha}, \delta \mathbf{u} ; \delta \boldsymbol{\psi}^{(1)} ; \delta \boldsymbol{\psi}_{1, i_{1}}^{(2)}, \delta \boldsymbol{\psi}_{2, i_{1}}^{(2)}\right)=0, \mathbf{x} \in \partial \Omega_{x}
$$

The following definitions were used in Equations (138) and (139):

$$
\begin{aligned}
& \mathbf{F}_{11}^{(3)}(\boldsymbol{\alpha}, \mathbf{u}) \triangleq \mathbf{A}_{11}^{(1)}(\boldsymbol{\alpha}, \mathbf{u}), \quad \mathbf{F}_{12}^{(3)}(\boldsymbol{\alpha}, \mathbf{u}) \triangleq \mathbf{A}_{21}^{(2)}(\boldsymbol{\alpha}, \mathbf{u}), \quad \mathbf{F}_{13}^{(3)}\left(\boldsymbol{\alpha}, \mathbf{u} ; \boldsymbol{\psi}^{(1)}\right) \triangleq-\frac{\partial \mathbf{\Theta}_{2, i_{1}}^{(2)}\left(\boldsymbol{\alpha}, \mathbf{u} ; \boldsymbol{\psi}^{(1)}\right)}{\partial \boldsymbol{\psi}^{(1)}}, \\
& \mathbf{F}_{14}^{(3)}\left(\boldsymbol{\alpha}, \mathbf{u} ; \boldsymbol{\psi}^{(1)} ; \boldsymbol{\psi}_{1, i_{1}}^{(2)}, \boldsymbol{\psi}_{2, i_{1}}^{(2)}\right) \triangleq \frac{\partial}{\partial \mathbf{u}}\left[\mathbf{A}_{21}^{(2)}(\boldsymbol{\alpha}, \mathbf{u}) \boldsymbol{\psi}_{1, i_{1}}^{(2)}+\mathbf{A}_{11}^{(1)}(\boldsymbol{\alpha}, \mathbf{u}) \boldsymbol{\psi}_{2, i_{1}}^{(2)}-\boldsymbol{\Theta}_{2, i_{1}}^{(2)}\left(\boldsymbol{\alpha}, \mathbf{u} ; \boldsymbol{\psi}^{(1)}\right)\right] \text {, } \\
& \mathbf{F}_{22}^{(3)}(\boldsymbol{\alpha}, \mathbf{u}) \triangleq \mathbf{F}_{11}^{(1)}(\boldsymbol{\alpha}, \mathbf{u}), \quad \mathbf{F}_{23}^{(3)}\left(\boldsymbol{\alpha}, \mathbf{u} ; \boldsymbol{\psi}^{(1)}\right) \triangleq-\frac{\partial \Theta_{1, i_{1}}^{(2)}\left(\boldsymbol{\alpha}, \mathbf{u} ; \boldsymbol{\psi}^{(1)}\right)}{\partial \boldsymbol{\psi}^{(1)}}, \\
& \mathbf{F}_{24}^{(3)}(\boldsymbol{\alpha}, \mathbf{u}) \triangleq \frac{\partial}{\partial \mathbf{u}}\left[\mathbf{F}_{11}^{(1)}(\boldsymbol{\alpha}, \mathbf{u}) \boldsymbol{\psi}_{1, i_{1}}^{(2)}-\boldsymbol{\Theta}_{1, i_{1}}^{(2)}\left(\boldsymbol{\alpha}, \mathbf{u} ; \boldsymbol{\psi}^{(1)}\right)\right] \text {, } \\
& \mathbf{Q}_{1, i_{1}}^{(3)}\left(\boldsymbol{\alpha}, \mathbf{u} ; \boldsymbol{\Psi}^{(1)} ; \boldsymbol{\Psi}_{1, i_{1}}^{(2)}, \boldsymbol{\Psi}_{2, i_{1}}^{(2)} ; \delta \boldsymbol{\alpha}\right) \triangleq \frac{\partial}{\partial \boldsymbol{\alpha}}\left[-\mathbf{A}_{11}^{(1)}(\boldsymbol{\alpha}, \mathbf{u}) \boldsymbol{\Psi}_{2, i_{1}}^{(2)}-\mathbf{A}_{21}^{(2)}(\boldsymbol{\alpha}, \mathbf{u}) \boldsymbol{\Psi}_{1, i_{1}}^{(2)}+\boldsymbol{\Theta}_{2, i_{1}}^{(2)}\left(\boldsymbol{\alpha}, \mathbf{u} ; \boldsymbol{\psi}^{(1)}\right)\right] \delta \boldsymbol{\alpha}, \\
& \mathbf{Q}_{2, i_{1}}^{(3)}\left(\boldsymbol{\alpha}, \mathbf{u} ; \boldsymbol{\psi}^{(1)} ; \boldsymbol{\Psi}_{1, i_{1}}^{(2)} ; \delta \boldsymbol{\alpha}\right) \triangleq \frac{\partial}{\partial \boldsymbol{\alpha}}\left[-\mathbf{F}_{11}^{(1)}(\boldsymbol{\alpha}, \mathbf{u}) \boldsymbol{\Psi}_{1, i_{1}}^{(2)}+\boldsymbol{\Theta}_{1, i_{1}}^{(2)}\left(\boldsymbol{\alpha}, \mathbf{u} ; \boldsymbol{\psi}^{(1)}\right)\right] \delta \boldsymbol{\alpha} .
\end{aligned}
$$

In order to obtain a well-posed system of equations for the vectors of variations $\delta \mathbf{u}$, $\delta \boldsymbol{\psi}^{(1)}, \delta \boldsymbol{\psi}_{1, i_{1}}^{(2)}$, and $\delta \boldsymbol{\psi}_{2, i_{1}}^{(2)}$ as well-determined unique functions of the parameter variations $\delta \alpha$, Equations (138)-(140) will be augmented by the equations underlying the 2nd-LVSS, namely Equations (A38) and (A39) in the Appendix A. The resulting system of equations can be written in the following block-matrix form:

$$
\begin{aligned}
& \left\{\left[\begin{array}{cccc}
\mathbf{F}_{11}^{(3)}(\boldsymbol{\alpha}, \mathbf{u}) & \mathbf{F}_{12}^{(3)}(\boldsymbol{\alpha}, \mathbf{u}) & \mathbf{F}_{13}^{(3)}\left(\boldsymbol{\alpha}, \mathbf{u} ; \boldsymbol{\psi}^{(1)}\right) & \mathbf{F}_{14}^{(3)}\left(\boldsymbol{\alpha}, \mathbf{u} ; \boldsymbol{\psi}^{(1)}\right) \\
0 & \mathbf{F}_{22}^{(3)}(\boldsymbol{\alpha}, \mathbf{u}) & \mathbf{F}_{23}^{(3)}\left(\boldsymbol{\alpha}, \mathbf{u} ; \boldsymbol{\psi}^{(1)}\right) & \mathbf{F}_{24}^{(3)}(\boldsymbol{\alpha}, \mathbf{u}) \\
0 & 0 & \mathbf{F}_{11}^{(2)}(\boldsymbol{\alpha}, \mathbf{u}) & \mathbf{F}_{12}^{(2)}(\boldsymbol{\alpha}, \mathbf{u}) \\
0 & 0 & 0 & \mathbf{F}_{11}^{(1)}(\boldsymbol{\alpha}, \mathbf{u})
\end{array}\right]\right\}_{\left(\boldsymbol{\alpha}^{0}, \mathbf{u}^{0}, \boldsymbol{\psi}^{(1,0)}\right)}\left[\begin{array}{c}
\delta \boldsymbol{\psi}_{2, i_{1}}^{(2)} \\
\delta \boldsymbol{\psi}_{1, i_{1}}^{(2)} \\
\delta \boldsymbol{\psi}^{(1)} \\
\delta \mathbf{u}
\end{array}\right] \\
& =\left[\begin{array}{c}
\mathbf{Q}_{1, i_{1}}^{(3)}\left(\boldsymbol{\alpha}^{0}, \mathbf{u}^{0} ; \boldsymbol{\psi}^{(1,0)} ; \boldsymbol{\psi}_{1, i_{1}}^{(2,0)}, \boldsymbol{\psi}_{2, i_{1}}^{(2,0)} ; \delta \boldsymbol{\alpha}\right) \\
\mathbf{Q}_{2, i_{1}}^{(3)}\left(\boldsymbol{\alpha}^{0}, \mathbf{u}^{0} ; \boldsymbol{\psi}^{(1,0)} ; \boldsymbol{\psi}_{1, i_{1}}^{(2,0)} ; \delta \boldsymbol{\alpha}\right) \\
\mathbf{Q}^{(2)}\left(\boldsymbol{\alpha}^{0}, \mathbf{u}^{0} ; \boldsymbol{\psi}^{(1,0)} ; \delta \boldsymbol{\alpha}\right) \\
\mathbf{Q}^{(1)}\left(\boldsymbol{\alpha}^{0}, \mathbf{u}^{0} ; \delta \boldsymbol{\alpha}\right)
\end{array}\right]
\end{aligned}
$$


together with the 3rd-level forward variational boundary conditions

$$
\begin{aligned}
& \mathbf{B}_{F}^{(3)}\left(\boldsymbol{\alpha}^{0}, \boldsymbol{\varphi}_{i_{1}}^{(3,0)} ; \delta \boldsymbol{\alpha}, \delta \mathbf{u} ; \delta \boldsymbol{\psi}^{(1)} ; \delta \boldsymbol{\psi}_{1, i_{1}}^{(2)}, \delta \boldsymbol{\psi}_{2, i_{1}}^{(2)}\right) \\
& \triangleq\left[\begin{array}{c}
\delta \mathbf{B}_{A}^{(2)}\left(\boldsymbol{\alpha}^{0}, \boldsymbol{\varphi}_{i_{1}}^{(3,0)} ; \delta \boldsymbol{\alpha}, \delta \mathbf{u} ; \delta \boldsymbol{\psi}^{(1)} ; \delta \boldsymbol{\psi}_{1, i_{1}}^{(2)}, \delta \boldsymbol{\psi}_{2, i_{1}}^{(2)}\right) \\
\delta \mathbf{B}_{A}^{(1)}\left(\boldsymbol{\alpha}^{0}, \mathbf{u}^{0} ; \boldsymbol{\psi}^{(1,0)} ; \delta \boldsymbol{\alpha}, \delta \mathbf{u}, \delta \boldsymbol{\psi}^{(1)}\right) \\
\mathbf{B}_{F}^{(1)}\left(\boldsymbol{\alpha}^{0}, \mathbf{u}^{0} ; \delta \boldsymbol{\alpha}, \delta \mathbf{u}\right)
\end{array}\right]=0, \quad \mathbf{x} \in \partial \Omega_{x}
\end{aligned}
$$

The system comprising Equations (145) and (146) constitute the 3rd-Level Variational Sensitivity System (3rd-LVSS), which can be solved, in principle, to obtain the variations $\delta \mathbf{u}, \delta \boldsymbol{\psi}^{(1)}, \delta \boldsymbol{\psi}_{1, i_{1}}^{(2)}$, and $\delta \boldsymbol{\psi}_{2, i_{1}}^{(2)}$ as functions of the parameter variations $\delta \boldsymbol{\alpha}$. Subsequently, the variations $\delta \mathbf{u}, \delta \boldsymbol{\psi}^{(1)}, \delta \boldsymbol{\psi}_{1, i_{1}}^{(2)}$, and $\delta \boldsymbol{\psi}_{2, i_{1}}^{(2)}$ could be replaced in Equation (137) to determine the indirect effect term $\left(\delta R_{i_{1} i_{2}}^{(2)}\right)_{\text {indirect }}$. This procedure would be impractical to use for largescale systems since it would require $T P(T P+1)(T P+3) / 3$ ! large-scale computations. This number of computations can be significantly reduced by using the $3^{\text {rd }}$-Level Adjoint Sensitivity System (3rd-LASS), which will be constructed next by using the same guiding principles as those underlying the development of the 2nd-LASS, aiming at computing the indirect effect term $\left(\delta R_{i_{1} i_{2}}^{(2)}\right)_{\text {indirect }}$ in an alternative manner, which circumvents the need for computing explicitly the vectors of variations $\delta \mathbf{u}, \delta \boldsymbol{\psi}^{(1)}, \delta \boldsymbol{\psi}_{1, i_{1}}^{(2)}$, and $\delta \boldsymbol{\psi}_{i, i_{1}}^{(2)}$. For this purpose, the product Hilbert space denoted as $\mathrm{H}_{\psi}^{(3)}\left(\Omega_{x}\right) \triangleq 2 \otimes \mathrm{H}_{\psi}^{(2)}\left(\Omega_{x}\right)$ is introduced; the elements of $\mathrm{H}_{\psi}^{(3)}\left(\Omega_{x}\right)$ are block-vectors of the form $\boldsymbol{\psi}_{i_{1} i_{2}}^{(3)} \triangleq\left(\boldsymbol{\psi}_{1, i_{1} i_{2}}^{(3)}, \boldsymbol{\psi}_{2, i_{1} i_{2}}^{(3)}, \boldsymbol{\psi}_{3, i_{1} i_{2}}^{(3)}, \boldsymbol{\psi}_{4, i_{1} i_{2}}^{(3)}\right) \in$ $\mathrm{H}_{\psi}^{(3)}\left(\Omega_{x}\right)$. The inner product between any two vectors $\mathbf{a}_{i_{1} i_{2}}^{(3)} \triangleq\left(\mathbf{a}_{1, i_{1} i_{2}}^{(3)}, \mathbf{a}_{2, i_{1} i_{2}}^{(3)}, \mathbf{a}_{3, i_{1} i_{2}}^{(3)}, \mathbf{a}_{4, i_{1} i_{2}}^{(3)}\right) \in$ $\mathrm{H}_{\psi}^{(3)}\left(\Omega_{x}\right)$ and $\mathbf{b}_{i_{1} i_{2}}^{(3)} \triangleq\left(\mathbf{b}_{1, i_{1} i_{2}}^{(3)}, \mathbf{b}_{2, i_{1} i_{2}}^{(3)}, \mathbf{b}_{3, i_{1} i_{2}}^{(3)}, \mathbf{b}_{4, i_{1} i_{2}}^{(3)}\right) \in \mathrm{H}_{\psi}^{(3)}\left(\Omega_{x}\right)$ in $\mathrm{H}_{\psi}^{(3)}\left(\Omega_{x}\right)$ will be denoted as $\langle\bullet, \bullet\rangle_{\psi^{(3)}}$, and is defined as follows:

$$
\left\langle\mathbf{a}_{i_{1} i_{2}}^{(3)}, \mathbf{b}_{i_{1} i_{2}}^{(3)}\right\rangle_{\psi^{(2)}} \triangleq \sum_{j=1}^{4} \int_{\Omega_{x}}\left(\mathbf{a}_{j, i_{1} i_{2}}^{(3)} \cdot \mathbf{b}_{j, i_{1} i_{2}}^{(3)}\right) d \mathbf{x}
$$

Using the above definition, form the inner product of Equation (145) with a yet undefined vector $\boldsymbol{\psi}_{i_{1} i_{2}}^{(3)} \triangleq\left(\boldsymbol{\psi}_{1, i_{1} i_{2}}^{(3)}, \boldsymbol{\psi}_{2, i_{1} i_{2}}^{(3)}, \boldsymbol{\psi}_{3, i_{1} i_{2}}^{(3)}, \boldsymbol{\psi}_{4, i_{1} i_{2}}^{(3)}\right) \in \mathrm{H}_{\psi}^{(3)}\left(\Omega_{x}\right)$ to obtain:

$$
\begin{aligned}
& \left\langle\left[\begin{array}{c}
\boldsymbol{\psi}_{1, i_{1} i_{2}}^{(3)} \\
\boldsymbol{\psi}_{2, i_{1} i_{2}}^{(3)} \\
\boldsymbol{\psi}_{3, i_{1} i_{2}}^{(3)} \\
\boldsymbol{\psi}_{4, i_{1} i_{2}}^{(3)}
\end{array}\right]^{\dagger},\left\{\left[\begin{array}{cccc}
\mathbf{F}_{11}^{(3)}(\boldsymbol{\alpha}, \mathbf{u}) & \mathbf{F}_{12}^{(3)}(\boldsymbol{\alpha}, \mathbf{u}) & \mathbf{F}_{13}^{(3)}\left(\boldsymbol{\alpha}, \mathbf{u} ; \boldsymbol{\psi}^{(1)}\right) & \mathbf{F}_{14}^{(3)}\left(\boldsymbol{\alpha}, \mathbf{u} ; \boldsymbol{\psi}^{(1)}\right) \\
0 & \mathbf{F}_{22}^{(3)}(\boldsymbol{\alpha}, \mathbf{u}) & \mathbf{F}_{23}^{(3)}\left(\boldsymbol{\alpha}, \mathbf{u} ; \boldsymbol{\psi}^{(1)}\right) & \mathbf{F}_{24}^{(3)}(\boldsymbol{\alpha}, \mathbf{u}) \\
0 & 0 & \mathbf{F}_{11}^{(2)}(\boldsymbol{\alpha}, \mathbf{u}) & \mathbf{F}_{12}^{(2)}(\boldsymbol{\alpha}, \mathbf{u}) \\
0 & 0 & 0 & \mathbf{F}_{11}^{(1)}(\boldsymbol{\alpha}, \mathbf{u})
\end{array}\right]\right\}_{\left(\boldsymbol{\alpha}^{0}, \mathbf{u}^{0}, \boldsymbol{\psi}^{(1,0)}\right)}\left[\begin{array}{c}
\delta \boldsymbol{\psi}_{2, i_{1}}^{(2)} \\
\delta \boldsymbol{\psi}_{1, i_{1}}^{(2)} \\
\delta \boldsymbol{\psi}^{(1)} \\
\delta \mathbf{u}
\end{array}\right]\right\rangle_{\psi^{(3)}} \\
& =\int_{\Omega_{x}}\left[\boldsymbol{\psi}_{1, i_{1} i_{2}}^{(3)} \cdot \mathbf{Q}_{1, i_{1}}^{(3)}\left(\boldsymbol{\alpha}^{0}, \mathbf{u}^{0} ; \boldsymbol{\psi}^{(1,0)} ; \boldsymbol{\psi}_{1, i_{1}}^{(2,0)}, \boldsymbol{\psi}_{2, i_{1}}^{(2,0)} ; \delta \boldsymbol{\alpha}\right)+\boldsymbol{\psi}_{2, i_{1} i_{2}}^{(3)} \cdot \mathbf{Q}_{2, i_{1}}^{(3)}\left(\boldsymbol{\alpha}^{0}, \mathbf{u}^{0} ; \boldsymbol{\psi}^{(1,0)} ; \boldsymbol{\psi}_{1, i_{1}}^{(2,0)} ; \delta \boldsymbol{\alpha}\right)\right. \\
& \left.+\boldsymbol{\psi}_{3, i_{1} i_{2}}^{(3)} \cdot \mathbf{Q}^{(2)}\left(\boldsymbol{\alpha}^{0}, \mathbf{u}^{0} ; \boldsymbol{\psi}^{(1,0)} ; \delta \boldsymbol{\alpha}\right)+\boldsymbol{\psi}_{4, i_{1} i_{2}}^{(3)} \cdot \mathbf{Q}^{(1)}\left(\boldsymbol{\alpha}^{0}, \mathbf{u}^{0} ; \delta \boldsymbol{\alpha}\right)\right] d \mathbf{x} \text {. }
\end{aligned}
$$

The inner product on the left-side of Equation (148) is now further transformed by using the definition of the adjoint operator to obtain the following relation: 


$$
\begin{aligned}
& \left\{\left\langle\left[\begin{array}{c}
\boldsymbol{\psi}_{1, i_{1} i_{2}}^{(3)} \\
\boldsymbol{\psi}_{2, i_{1} i_{2}}^{(3)} \\
\boldsymbol{\psi}_{3, i_{1} i_{2}}^{(3)} \\
\boldsymbol{\psi}_{4, i_{1} i_{2}}^{(3)}
\end{array}\right]^{\dagger},\left[\begin{array}{cccc}
\mathbf{F}_{11}^{(3)} & \mathbf{F}_{12}^{(3)} & \mathbf{F}_{13}^{(3)} & \mathbf{F}_{14}^{(3)} \\
0 & \mathbf{F}_{22}^{(3)} & \mathbf{F}_{23}^{(3)} & \mathbf{F}_{24}^{(3)} \\
0 & 0 & \mathbf{F}_{11}^{(2)} & \mathbf{F}_{12}^{(2)} \\
0 & 0 & 0 & \mathbf{F}_{11}^{(1)}
\end{array}\right]\left[\begin{array}{c}
\delta \boldsymbol{\psi}_{2, i_{1}}^{(2)} \\
\delta \boldsymbol{\psi}_{1, i_{1}}^{(2)} \\
\delta \boldsymbol{\psi}^{(1)} \\
\delta \mathbf{u}
\end{array}\right]\right\rangle_{\psi^{(3)}}\right\}_{\boldsymbol{\alpha}^{0}}
\end{aligned}
$$

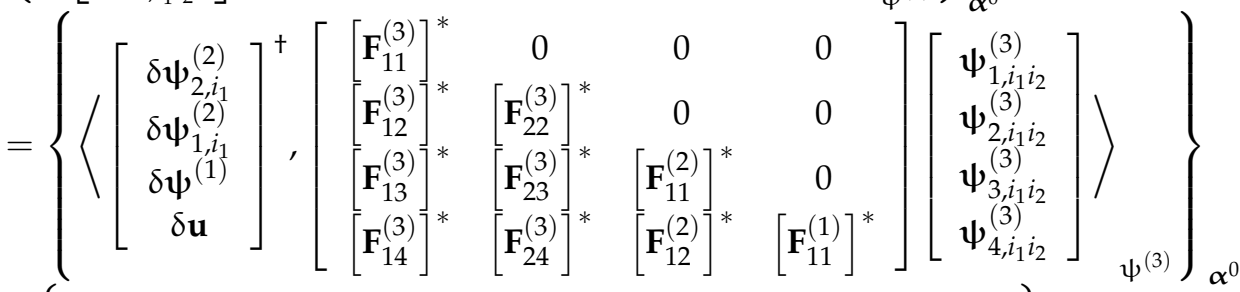

$$
\begin{aligned}
& +\left\{\left\{P^{(3)}\left[\boldsymbol{\alpha} ; \mathbf{u} ; \boldsymbol{\psi}^{(1)} ; \boldsymbol{\psi}_{i_{1}}^{(2)}(\mathbf{x}) ; \boldsymbol{\psi}_{i_{1} i_{2}}^{(3)} ; \delta \boldsymbol{\alpha} ; \delta \mathbf{u} ; \delta \boldsymbol{\psi}^{(1)} ; \delta \boldsymbol{\psi}_{2, i_{1}}^{(2)}, \delta \boldsymbol{\psi}_{1, i_{1}}^{(2)}\right]\right\}_{\partial \Omega_{x}}\right\}_{\boldsymbol{\alpha}^{0}} \text {. }
\end{aligned}
$$

In Equation (149), the symbol [ ] indicates "adjoint," as before, while the quantity $\left\{P^{(3)}\left[\boldsymbol{\alpha} ; \mathbf{u} ; \boldsymbol{\psi}^{(1)} ; \boldsymbol{\psi}_{i_{1}}^{(2)}(\mathbf{x}) ; \boldsymbol{\psi}_{i_{1} i_{2}}^{(3)} ; \delta \boldsymbol{\alpha} ; \delta \mathbf{u} ; \delta \boldsymbol{\psi}^{(1)} ; \delta \boldsymbol{\psi}_{2, i_{1}}^{(2)}, \delta \boldsymbol{\psi}_{1, i_{1}}^{(2)}\right]\right\}_{\partial \Omega_{x}}$ denotes the corresponding bilinear concomitant on $\mathbf{x} \in \partial \Omega_{x}$; as before, the superscript "zero" denotes "nominal values" and the short-hand notation \{\}$_{\alpha^{0}}$ indicates that the quantity within the braces is to be evaluated at the nominal values of all parameters and (forward and adjoint) state functions.

The domains of the adjoint operators appearing on the right-side of Equation (149) can now be specified by requiring that the function $\boldsymbol{\psi}_{i_{1} i_{2}}^{(3)} \triangleq\left(\boldsymbol{\psi}_{1, i_{1} i_{2}}^{(3)}, \boldsymbol{\psi}_{2, i_{1} i_{2}}^{(3)}, \boldsymbol{\psi}_{3, i_{1} i_{2}}^{(3)}, \boldsymbol{\psi}_{4, i_{1} i_{2}}^{(3)}\right) \in$ $\mathrm{H}_{\psi}^{(3)}\left(\Omega_{x}\right)$ can now be further specified by requiring that it satisfies the following $3 r d$-level adjoint boundary conditions, represented in operator form as follows:

$$
\left\{\mathbf{B}_{A}^{(3)}\left(\boldsymbol{\alpha}, \mathbf{u} ; \boldsymbol{\psi}^{(1)} ; \boldsymbol{\psi}_{i_{1}}^{(2)} ; \boldsymbol{\psi}_{i_{1} i_{2}}^{(3)}\right)\right\}_{\boldsymbol{\alpha}^{0}}=0, \mathbf{x} \in \partial \Omega_{x}\left(\boldsymbol{\alpha}^{0}\right)
$$

The above adjoint boundary and/or initial conditions for $\boldsymbol{\psi}_{i_{1} i_{2}}^{(3)} \triangleq\left(\boldsymbol{\psi}_{1, i_{1} i_{2}}^{(3)}, \boldsymbol{\psi}_{2, i_{1} i_{2}}^{(3)}, \boldsymbol{\psi}_{3, i_{1} i_{2}}^{(3)}\right.$, $\left.\psi_{4, i_{1} i_{2}}^{(3)}\right) \in \mathrm{H}_{\psi}^{(3)}\left(\Omega_{x}\right)$ are determined by following the same principles as described in Section 2, namely that: (a) they must be independent of unknown values of $\delta \alpha, \delta \mathbf{u}, \delta \psi^{(1)}, \delta \psi_{1, i_{1}}^{(2)}$ and $\delta \Psi_{2, i_{1}}^{(2)}$; and (b) substituting Equations (146) and (150) into the expression of $\left\{P^{(3)}\left[\boldsymbol{\alpha} ; \mathbf{u} ; \boldsymbol{\psi}^{(1)} ; \boldsymbol{\psi}_{i_{1}}^{(2)}(\mathbf{x}) ; \boldsymbol{\psi}_{i_{1} i_{2}}^{(3)} ; \delta \boldsymbol{\alpha} ; \delta \mathbf{u} ; \delta \boldsymbol{\psi}^{(1)} ; \delta \boldsymbol{\psi}_{2, i_{1}}^{(2)}, \delta \boldsymbol{\psi}_{1, i_{1}}^{(2)}\right]\right\}_{\partial \Omega_{x}}$ must cause all terms containing unknown values of $\delta \mathbf{u} \delta \boldsymbol{\psi}^{(1)}, \delta \boldsymbol{\psi}_{2, i_{1}}^{(2)}, \delta \boldsymbol{\psi}_{1, i_{1}}^{(2)}$ to vanish, which will reduce the bilinear concomitant to an expression that will contain only known values of $\delta \alpha$; this expression will be denoted as $\hat{P}^{(3)}\left[\boldsymbol{\alpha} ; \mathbf{u} ; \boldsymbol{\psi}^{(1)} ; \boldsymbol{\psi}_{i_{1}}^{(2)}(\mathbf{x}) ; \boldsymbol{\psi}_{i_{1} i_{2}}^{(3)} ; \delta \boldsymbol{\alpha}\right]$. In general, $\hat{P}^{(3)}\left[\boldsymbol{\alpha} ; \mathbf{u} ; \boldsymbol{\psi}^{(1)} ; \boldsymbol{\psi}_{i_{1}}^{(2)}(\mathbf{x}) ; \boldsymbol{\psi}_{i_{1} i_{2}}^{(3)} ; \delta \boldsymbol{\alpha}\right]$ does not automatically vanish as a result of these operations. 
Implementing the 3rd-level variational boundary/initial conditions, cf. Equation (146), together with the 3rd-level adjoint boundary/initial conditions represented in (150) into Equation (149) will transform the later into the form

$$
\begin{aligned}
& \left.\left\{\left\langle\left[\begin{array}{c}
\delta \boldsymbol{\psi}_{2, i_{1}}^{(2)} \\
\delta \boldsymbol{\psi}_{1, i_{1}}^{(2)} \\
\delta \boldsymbol{\psi}^{(1)} \\
\delta \mathbf{u}
\end{array}\right]^{+},\left[\begin{array}{cccc}
{\left[\mathbf{F}_{11}^{(3)}\right]^{*}} & 0 & 0 & 0 \\
{\left[\mathbf{F}_{12}^{(3)}\right]^{*}} & {\left[\mathbf{F}_{22}^{(3)}\right]^{*}} & 0 & 0 \\
{\left[\mathbf{F}_{13}^{(3)}\right]^{*}} & {\left[\mathbf{F}_{23}^{(3)}\right]^{*}} & {\left[\mathbf{F}_{11}^{(2)}\right]^{*}} & 0 \\
{\left[\mathbf{F}_{14}^{(3)}\right]^{*}} & {\left[\mathbf{F}_{24}^{(3)}\right]^{*}} & {\left[\mathbf{F}_{12}^{(2)}\right]^{*}} & {\left[\mathbf{F}_{11}^{(1)}\right]^{*}}
\end{array}\right]\left[\begin{array}{c}
\boldsymbol{\psi}_{1, i_{1} i_{2}}^{(3)} \\
\boldsymbol{\psi}_{2, i_{1} i_{2}}^{(3)} \\
\boldsymbol{\psi}_{3, i_{1} i_{2}}^{(3)} \\
\boldsymbol{\psi}_{4, i_{1} i_{2}}^{(3)}
\end{array}\right]\right\rangle\right\}_{\boldsymbol{\psi}^{(3)}}\right\}_{\boldsymbol{\alpha}^{0}} \\
& =\left\{\left\langle\left[\begin{array}{c}
\boldsymbol{\psi}_{1, i_{1} i_{2}}^{(3)} \\
\boldsymbol{\Psi}_{2, i_{1} i_{2}}^{(3)} \\
\boldsymbol{\Psi}_{3, i_{1} i_{2}}^{(3)} \\
\boldsymbol{\psi}_{4, i_{1} i_{2}}^{(3)}
\end{array}\right]^{\dagger}\left[\begin{array}{cccc}
\mathbf{F}_{11}^{(3)} & \mathbf{F}_{12}^{(3)} & \mathbf{F}_{13}^{(3)} & \mathbf{F}_{14}^{(3)} \\
0 & \mathbf{F}_{22}^{(3)} & \mathbf{F}_{23}^{(3)} & \mathbf{F}_{24}^{(3)} \\
0 & 0 & \mathbf{F}_{11}^{(2)} & \mathbf{F}_{12}^{(2)} \\
0 & 0 & 0 & \mathbf{F}_{11}^{(1)}
\end{array}\right]\left[\begin{array}{c}
\delta \boldsymbol{\psi}_{2, i_{1}}^{(2)} \\
\delta \boldsymbol{\psi}_{1, i_{1}}^{(2)} \\
\delta \boldsymbol{\psi}^{(1)} \\
\delta \mathbf{u}
\end{array}\right]\right\rangle_{\psi^{(3)}}\right\}_{\boldsymbol{\alpha}^{0}} \\
& -\left\{\hat{P}^{(3)}\left[\boldsymbol{\alpha} ; \mathbf{u} ; \boldsymbol{\psi}^{(1)} ; \boldsymbol{\psi}_{i_{1}}^{(2)}(\mathbf{x}) ; \boldsymbol{\psi}_{i_{1} i_{2}}^{(3)} ; \delta \boldsymbol{\alpha}\right]\right\}_{\boldsymbol{\alpha}^{0}} .
\end{aligned}
$$

The term on the left-side of Equation (151) is replaced by the right-side of Equation (148) to obtain the following relation:

$$
\begin{aligned}
& \left.\left\{\left\langle\left[\begin{array}{c}
\delta \boldsymbol{\psi}_{2, i_{1}}^{(2)} \\
\delta \boldsymbol{\psi}_{1, i_{1}}^{(2)} \\
\delta \boldsymbol{\psi}^{(1)} \\
\delta \mathbf{u}
\end{array}\right]^{\dagger},\left[\begin{array}{cccc}
{\left[\mathbf{F}_{11}^{(3)}\right]^{*}} & 0 & 0 & 0 \\
{\left[\mathbf{F}_{12}^{(3)}\right]^{*}} & {\left[\mathbf{F}_{22}^{(3)}\right]^{*}} & 0 & 0 \\
{\left[\mathbf{F}_{13}^{(3)}\right]^{*}} & {\left[\mathbf{F}_{23}^{(3)}\right]^{*}} & {\left[\mathbf{F}_{11}^{(2)}\right]^{*}} & 0 \\
{\left[\mathbf{F}_{14}^{(3)}\right]^{*}} & {\left[\mathbf{F}_{24}^{(3)}\right]^{*}} & {\left[\mathbf{F}_{12}^{(2)}\right]^{*}} & {\left[\mathbf{F}_{11}^{(1)}\right]^{*}}
\end{array}\right]\left[\begin{array}{c}
\boldsymbol{\psi}_{1, i_{1} i_{2}}^{(3)} \\
\boldsymbol{\psi}_{2, i_{1} i_{2}}^{(3)} \\
\boldsymbol{\psi}_{3, i_{1} i_{2}}^{(3)} \\
\boldsymbol{\psi}_{4, i_{1} i_{2}}^{(3)}
\end{array}\right]\right\rangle\right\}_{\psi_{(3)}^{(3)}}\right\}_{\boldsymbol{\alpha}^{0}} \\
& =\left\{\left\langle\left[\begin{array}{c}
\psi_{1, i_{1} i_{2}}^{(3)} \\
\boldsymbol{\psi}_{2, i_{1} i_{2}}^{(3)} \\
\boldsymbol{\psi}_{3, i_{1} i_{2}}^{(3)} \\
\boldsymbol{\psi}_{4, i_{1} i_{2}}^{(3)}
\end{array}\right]^{\dagger}\left[\begin{array}{c}
\mathbf{Q}_{1, i_{1}}^{(3)}\left(\boldsymbol{\alpha}^{0}, \mathbf{u}^{0} ; \boldsymbol{\psi}^{(1,0)} ; \boldsymbol{\psi}_{1, i_{1}}^{(2,0)}, \boldsymbol{\psi}_{2, i_{1}}^{(2,0)} ; \delta \boldsymbol{\alpha}\right) \\
\mathbf{Q}_{2, i_{1}}^{(3)}\left(\boldsymbol{\alpha}^{0}, \mathbf{u}^{0} ; \boldsymbol{\psi}^{(1,0)} ; \boldsymbol{\psi}_{1, i_{1}}^{(2,0)} ; \boldsymbol{\alpha}\right) \\
\mathbf{Q}^{(2)}\left(\boldsymbol{\alpha}^{0}, \mathbf{u}^{0} ; \boldsymbol{\psi}^{(1,0)} ; \delta \boldsymbol{\alpha}\right) \\
\mathbf{Q}^{(1)}\left(\boldsymbol{\alpha}^{0}, \mathbf{u}^{0} ; \delta \boldsymbol{\alpha}\right)
\end{array}\right]\right\rangle_{\psi^{(3)}}\right\}_{\boldsymbol{\alpha}^{0}} \\
& -\left\{\hat{P}^{(3)}\left[\boldsymbol{\alpha} ; \mathbf{u} ; \boldsymbol{\psi}^{(1)} ; \boldsymbol{\psi}_{i_{1}}^{(2)}(\mathbf{x}) ; \boldsymbol{\psi}_{i_{1} i_{2}}^{(3)} ; \delta \boldsymbol{\alpha}\right]\right\}_{\boldsymbol{\alpha}^{0}} .
\end{aligned}
$$

The definition of the 3rd-level adjoint function $\boldsymbol{\psi}_{i_{1} i_{2}}^{(3)} \triangleq\left(\boldsymbol{\psi}_{1, i_{1} i_{2}}^{(3)} \boldsymbol{\psi}_{2, i_{1} i_{2}}^{(3)}, \boldsymbol{\psi}_{3, i_{1} i_{2} i_{2}}^{(3)}, \boldsymbol{\psi}_{4, i_{1} i_{2}}^{(3)}\right) \in$ $\mathrm{H}_{\psi}^{(3)}\left(\Omega_{x}\right)$ will now be completed by requiring that the left-side of Equation (152) represent the indirect effect term $\left(\delta R_{i_{1} i_{2}}^{(2)}\right)_{\text {indirect }}$ defined in Equation (137), which yields the following relationship:

$$
\begin{aligned}
& \left.\left\{\left\langle\left[\begin{array}{c}
\delta \boldsymbol{\psi}_{2, i_{1}}^{(2)} \\
\delta \boldsymbol{\psi}_{1, i_{1}}^{(2)} \\
\delta \boldsymbol{\psi}^{(1)} \\
\delta \mathbf{u}
\end{array}\right]^{+}\left[\begin{array}{cccc}
{\left[\mathbf{F}_{11}^{(3)}\right]^{*}} & 0 & 0 & 0 \\
{\left[\mathbf{F}_{12}^{(3)}\right]^{*}} & {\left[\mathbf{F}_{22}^{(3)}\right]^{*}} & 0 & 0 \\
{\left[\mathbf{F}_{13}^{(3)}\right]^{*}} & {\left[\mathbf{F}_{23}^{(3)}\right]^{*}} & {\left[\mathbf{F}_{11}^{(2)}\right]^{*}} & 0 \\
{\left[\mathbf{F}_{14}^{(3)}\right]^{*}} & {\left[\mathbf{F}_{24}^{(3)}\right]^{*}} & {\left[\mathbf{F}_{12}^{(2)}\right]^{*}} & {\left[\mathbf{F}_{11}^{(1)}\right]^{*}}
\end{array}\right]\left[\begin{array}{c}
\boldsymbol{\psi}_{1, i_{1} i_{2}}^{(3)} \\
\boldsymbol{\psi}_{2, i_{1} i_{2}} \\
\boldsymbol{\psi}_{3, i_{1} i_{2}}^{(3)} \\
\boldsymbol{\psi}_{3, i_{1} i_{2}}^{(3)}
\end{array}\right]\right\rangle\right\}_{\boldsymbol{\psi}^{(3)}}\right\}_{\boldsymbol{\alpha}^{0}} \\
& =\left\{\left\langle\left(\delta \boldsymbol{\psi}_{2, i_{1}}^{(2)}, \delta \boldsymbol{\psi}_{1, i_{1}}^{(2)} \delta \boldsymbol{\psi}^{(1)}, \delta \mathbf{u}\right),\left[\begin{array}{c}
\partial R_{i_{1} i_{2}}^{(2)} / \partial \boldsymbol{\psi}_{2, i_{1}}^{(2)} \\
\partial R_{i_{1} i_{2}}^{(2)} / \partial \boldsymbol{\psi}_{1, i_{1}}^{(2)} \\
\partial R_{i_{1} i_{2}}^{(2)} / \partial \boldsymbol{\psi}^{(1)} \\
\partial R_{i_{1} i_{2}}^{(2)} / \partial \mathbf{u}
\end{array}\right]\right\rangle_{\psi^{(3)}}\right\}_{\boldsymbol{\alpha}^{0}} \text {. }
\end{aligned}
$$


The relation indicated in Equation (153) implies that the adjoint function $\psi_{i_{1} i_{2}}^{(3)} \triangleq$ $\left(\boldsymbol{\psi}_{1, i_{1} i_{2}}^{(3)}, \boldsymbol{\psi}_{2, i_{1} i_{2},}^{(3)}, \boldsymbol{\psi}_{3, i_{1} i_{2}}^{(3)} \boldsymbol{\psi}_{4, i_{1} i_{2}}^{(3)}\right) \in \mathrm{H}_{\psi}^{(3)}\left(\Omega_{x}\right)$ is the weak solution (in the sense of distributions) of the following operator equation:

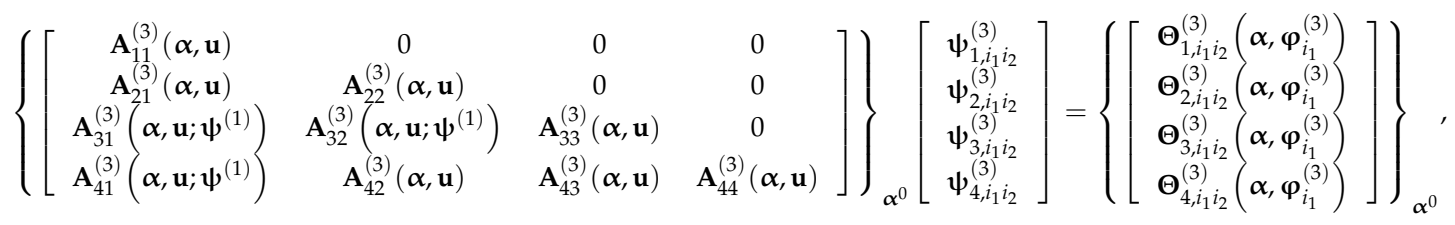

where the 3rd-level adjoint operators $\mathbf{A}_{i j}^{(3)}, i, j=1,2,3,4$, and sources $\boldsymbol{\Theta}_{j, i_{1} i_{2}}^{(3)} j=1,2,3,4$, are defined as follows:

$$
\begin{aligned}
& \mathbf{A}_{11}^{(3)}(\boldsymbol{\alpha}, \mathbf{u}) \triangleq\left[\mathbf{F}_{11}^{(3)}(\boldsymbol{\alpha}, \mathbf{u})\right]^{*}, \mathbf{A}_{21}^{(3)}(\boldsymbol{\alpha}, \mathbf{u}) \triangleq\left[\mathbf{F}_{12}^{(3)}(\boldsymbol{\alpha}, \mathbf{u})\right]^{*}, \mathbf{A}_{22}^{(3)}(\boldsymbol{\alpha}, \mathbf{u}) \triangleq\left[\mathbf{F}_{22}^{(3)}(\boldsymbol{\alpha}, \mathbf{u})\right]^{*}, \\
& \mathbf{A}_{31}^{(3)}\left(\boldsymbol{\alpha}, \mathbf{u} ; \boldsymbol{\psi}^{(1)}\right) \triangleq\left[\mathbf{F}_{13}^{(3)}\left(\boldsymbol{\alpha}, \mathbf{u} ; \boldsymbol{\psi}^{(1)}\right)\right]^{*}, \mathbf{A}_{32}^{(3)}\left(\boldsymbol{\alpha}, \mathbf{u} ; \boldsymbol{\psi}^{(1)}\right) \triangleq\left[\mathbf{F}_{23}^{(3)}\left(\boldsymbol{\alpha}, \mathbf{u} ; \boldsymbol{\psi}^{(1)}\right)\right]^{*}, \\
& \mathbf{A}_{33}^{(3)}(\boldsymbol{\alpha}, \mathbf{u}) \triangleq\left[\mathbf{F}_{11}^{(2)}(\boldsymbol{\alpha}, \mathbf{u})\right]^{*}, \quad \mathbf{A}_{41}^{(3)}\left(\boldsymbol{\alpha}, \mathbf{u} ; \boldsymbol{\psi}^{(1)}\right) \triangleq\left[\mathbf{F}_{14}^{(3)}\left(\boldsymbol{\alpha}, \mathbf{u} ; \boldsymbol{\psi}^{(1)}\right)\right]^{*}, \\
& \mathbf{A}_{42}^{(3)}(\boldsymbol{\alpha}, \mathbf{u}) \triangleq\left[\mathbf{F}_{24}^{(3)}(\boldsymbol{\alpha}, \mathbf{u})\right]^{*}, \mathbf{A}_{43}^{(3)}(\boldsymbol{\alpha}, \mathbf{u}) \triangleq\left[\mathbf{F}_{12}^{(2)}(\boldsymbol{\alpha}, \mathbf{u})\right]^{*}, \mathbf{A}_{44}^{(3)}(\boldsymbol{\alpha}, \mathbf{u}) \triangleq\left[\mathbf{F}_{11}^{(1)}(\boldsymbol{\alpha}, \mathbf{u})\right]^{*} \text {. } \\
& \text { and } \\
& \boldsymbol{\Theta}_{1, i_{1} i_{2}}^{(3)}\left(\boldsymbol{\alpha}, \boldsymbol{\varphi}_{i_{1}}^{(3)}\right) \triangleq \frac{\partial}{\partial \boldsymbol{\psi}_{2, i_{1}}^{(2)}}\left[R_{i_{1} i_{2}}^{(2)}\left(\boldsymbol{\alpha}, \mathbf{u} ; \boldsymbol{\psi}^{(1)} ; \boldsymbol{\psi}_{1, i_{1}}^{(2)}, \boldsymbol{\psi}_{2, i_{1}}^{(2)}\right)\right], \\
& \boldsymbol{\Theta}_{2, i_{1} i_{2}}^{(3)}\left(\boldsymbol{\alpha}, \boldsymbol{\varphi}_{i_{1}}^{(3)}\right) \triangleq \frac{\partial}{\partial \boldsymbol{\psi}_{1, i_{1}}^{(2)}}\left[R_{i_{1} i_{2}}^{(2)}\left(\boldsymbol{\alpha}, \mathbf{u} ; \boldsymbol{\psi}^{(1)} ; \boldsymbol{\psi}_{1, i_{1}}^{(2)}, \boldsymbol{\psi}_{2, i_{1}}^{(2)}\right)\right] \text {, } \\
& \boldsymbol{\Theta}_{3, i_{1} i_{2}}^{(3)}\left(\boldsymbol{\alpha}, \boldsymbol{\varphi}_{i_{1}}^{(3)}\right) \triangleq \frac{\partial}{\partial \boldsymbol{\psi}^{(1)}}\left[R_{i_{1} i_{2}}^{(2)}\left(\boldsymbol{\alpha}, \mathbf{u} ; \boldsymbol{\psi}^{(1)} ; \boldsymbol{\psi}_{1, i_{1}}^{(2)}, \boldsymbol{\psi}_{2, i_{1}}^{(2)}\right)\right] \text {, } \\
& \boldsymbol{\Theta}_{4, i_{1} i_{2}}^{(3)}\left(\boldsymbol{\alpha}, \boldsymbol{\varphi}_{i_{1}}^{(3)}\right) \triangleq \frac{\partial}{\partial \mathbf{u}}\left[R_{i_{1} i_{2}}^{(2)}\left(\boldsymbol{\alpha}, \mathbf{u} ; \boldsymbol{\psi}^{(1)} ; \boldsymbol{\psi}_{1, i_{1}}^{(2)}, \boldsymbol{\psi}_{2, i_{1}}^{(2)}\right)\right] \text {. }
\end{aligned}
$$

Using the relations expressed in Equations (A48), (141) and (155) enables the recasting of Equation (154) the latter into the following computationally efficient form, for $i_{1}=$ $1, \ldots, T P ; i_{2}=1, \ldots, i_{1}$ :

$\mathbf{F}_{11}^{(1)}(\boldsymbol{\alpha}, \mathbf{u}) \boldsymbol{\psi}_{1, i_{1} i_{2}}^{(3)}=\boldsymbol{\Theta}_{1, i_{1} i_{2}}^{(3)}\left(\boldsymbol{\alpha}, \boldsymbol{\varphi}_{i_{1}}^{(3)}\right)$,

$\mathbf{A}_{11}^{(1)}(\boldsymbol{\alpha}, \mathbf{u}) \boldsymbol{\Psi}_{2, i_{1} i_{2}}^{(3)}=\mathbf{\Theta}_{2, i_{1} i_{2}}^{(3)}\left(\boldsymbol{\alpha}, \boldsymbol{\varphi}_{i_{1}}^{(3)}\right)-\mathbf{A}_{21}^{(3)}(\boldsymbol{\alpha}, \mathbf{u}) \boldsymbol{\Psi}_{1, i_{1} i_{2}}^{(3)}$

$\mathbf{F}_{11}^{(1)}(\boldsymbol{\alpha}, \mathbf{u}) \boldsymbol{\psi}_{3, i_{1} i_{2}}^{(3)}=\boldsymbol{\Theta}_{3, i_{1} i_{2}}^{(3)}\left(\boldsymbol{\alpha}, \boldsymbol{\varphi}_{i_{1}}^{(3)}\right)-\mathbf{A}_{31}^{(3)}\left(\boldsymbol{\alpha}, \mathbf{u} ; \boldsymbol{\psi}^{(1)}\right) \boldsymbol{\psi}_{1, i_{1} i_{2}}^{(3)}-\mathbf{A}_{32}^{(3)}\left(\boldsymbol{\alpha}, \mathbf{u} ; \boldsymbol{\psi}^{(1)}\right) \boldsymbol{\psi}_{2, i_{1} i_{2}}^{(3)}$

$\mathbf{A}_{11}^{(1)}(\boldsymbol{\alpha}, \mathbf{u}) \boldsymbol{\psi}_{4, i_{1} i_{2}}^{(3)}=\boldsymbol{\Theta}_{4, i_{1} i_{2}}^{(3)}\left(\boldsymbol{\alpha}, \boldsymbol{\varphi}_{i_{1}}^{(3)}\right)-\mathbf{A}_{41}^{(3)}\left(\boldsymbol{\alpha}, \mathbf{u} ; \boldsymbol{\psi}^{(1)}\right) \boldsymbol{\psi}_{1, i_{1} i_{2}}^{(3)}-\mathbf{A}_{42}^{(3)}(\boldsymbol{\alpha}, \mathbf{u}) \boldsymbol{\psi}_{2, i_{1} i_{2}}^{(3)}-\mathbf{A}_{43}^{(3)}(\boldsymbol{\alpha}, \mathbf{u}) \boldsymbol{\psi}_{3, i_{1} i_{2}}^{(3)}$.

The 3rd-level adjoint function $\psi_{i_{1} i_{2}}^{(3)} \triangleq\left(\psi_{1, i_{1} i_{2},}^{(3)}, \psi_{2, i_{1} i_{2},}^{(3)} \psi_{3, i_{1} i_{2}}^{(3)}, \psi_{4, i_{1} i_{2}}^{(3)}\right) \in \mathrm{H}_{\psi}^{(3)}\left(\Omega_{x}\right)$ is obtained by solving Equations (157) and (150), which will be called the 3rd-Level Adjoint Sensitivity System (3rd-LASS). In view of Equations (152)-(154), the "indirect effect term" $\left(\delta R_{i_{1} i_{2}}^{(2)}\right)_{\text {indirect }}$ defined in Equation (137) can now be expressed in terms of the 3rd-level adjoint function $\boldsymbol{\psi}_{i_{1} i_{2}}^{(3)} \triangleq\left(\boldsymbol{\psi}_{1, i_{1} i_{2}}^{(3)}, \boldsymbol{\psi}_{2, i_{1} i_{2}}^{(3)}, \boldsymbol{\psi}_{3, i_{1} i_{2}}^{(3)}, \boldsymbol{\psi}_{4, i_{1} i_{2}}^{(3)}\right) \in \mathrm{H}_{\psi}^{(3)}\left(\Omega_{x}\right)$ as follows:

$$
\begin{aligned}
& \left(\delta R_{i_{1} i_{2}}^{(2)}\right)_{\text {indirect }}=\left\{\int _ { \Omega _ { x } } \left[\boldsymbol{\psi}_{1, i_{1} i_{2}}^{(3)} \cdot \mathbf{Q}_{1, i_{1}}^{(3)}\left(\boldsymbol{\alpha}, \mathbf{u} ; \boldsymbol{\psi}^{(1)} ; \boldsymbol{\psi}_{1, i_{1},}^{(2)} \boldsymbol{\psi}_{2, i_{1}}^{(2)} ; \delta \boldsymbol{\alpha}\right)+\boldsymbol{\psi}_{2, i_{1} i_{2}}^{(3)} \cdot \mathbf{Q}_{2, i_{1}}^{(3)}\left(\boldsymbol{\alpha}, \mathbf{u} ; \boldsymbol{\psi}^{(1)} ; \boldsymbol{\psi}_{1, i_{1}}^{(2)} ; \delta \boldsymbol{\alpha}\right)\right.\right. \\
& \left.\left.+\boldsymbol{\psi}_{3, i_{1} i_{2}}^{(3)} \cdot \mathbf{Q}^{(2)}\left(\boldsymbol{\alpha}, \mathbf{u} ; \boldsymbol{\psi}^{(1)} ; \delta \boldsymbol{\alpha}\right)+\boldsymbol{\psi}_{4, i_{1} i_{2}}^{(3)} \cdot \mathbf{Q}^{(1)}(\boldsymbol{\alpha}, \mathbf{u} ; \delta \boldsymbol{\alpha})\right]\right\}_{\boldsymbol{\alpha}^{0}} d \mathbf{x}-\left\{\hat{P}^{(3)}\left[\boldsymbol{\alpha} ; \mathbf{u} ; \boldsymbol{\psi}^{(1)} ; \boldsymbol{\psi}_{i_{1}}^{(2)}(\mathbf{x}) ; \boldsymbol{\psi}_{i_{1} i_{2}}^{(3)} ; \delta \boldsymbol{\alpha}\right]\right\}_{\boldsymbol{\alpha}^{0}} .
\end{aligned}
$$

The quantities $\mathbf{Q}_{1, i_{1}}^{(3)}\left(\boldsymbol{\alpha}, \boldsymbol{\varphi}_{i_{1}}^{(3)} ; \delta \boldsymbol{\alpha}\right)$ and $\mathbf{Q}_{2, i_{1}}^{(3)}\left(\boldsymbol{\alpha}, \boldsymbol{\varphi}_{i_{1}}^{(3)} ; \delta \boldsymbol{\alpha}\right)$, defined in Equations (143) and (144), respectively, are linear in $\delta \alpha$. The residual bilinear concomitant 
$\hat{P}^{(3)}\left[\boldsymbol{\alpha} ; \mathbf{u} ; \boldsymbol{\psi}^{(1)} ; \boldsymbol{\psi}_{i_{1}}^{(2)}(\mathbf{x}) ; \boldsymbol{\psi}_{i_{1} i_{2}}^{(3)} ; \delta \boldsymbol{\alpha}\right]$ is also linear in $\delta \boldsymbol{\alpha}$. Therefore, these quantities can be written as follows:

$$
\begin{aligned}
& \mathbf{Q}_{1, i_{1}}^{(3)}\left(\boldsymbol{\alpha}, \boldsymbol{\varphi}_{i_{1}}^{(3)} ; \delta \boldsymbol{\alpha}\right)=\sum_{i_{3}=1}^{T P} \frac{\partial \mathbf{Q}_{1, i_{1}}^{(3)}\left(\boldsymbol{\alpha}, \boldsymbol{\varphi}_{i_{1}}^{(3)}\right)}{\partial \alpha_{i_{3}}} \delta \alpha_{i_{3},} \\
& \mathbf{Q}_{2, i_{1}}^{(3)}\left(\boldsymbol{\alpha}, \boldsymbol{\varphi}_{i_{1}}^{(3)} ; \delta \boldsymbol{\alpha}\right)=\sum_{i_{3}=1}^{T P} \frac{\partial \mathbf{Q}_{2, i_{1}}^{(3)}\left(\boldsymbol{\alpha}, \boldsymbol{\varphi}_{i_{1}}^{(3)}\right)}{\partial \alpha_{i_{3}}} \delta \alpha_{i_{3}},
\end{aligned}
$$

and

$$
\hat{P}^{(3)}\left(\boldsymbol{\alpha}, \boldsymbol{\varphi}_{i_{1}}^{(3)} ; \boldsymbol{\psi}_{1, i_{1} i_{2}}^{(3)}, \boldsymbol{\psi}_{2, i_{1} i_{2}}^{(3)}, \boldsymbol{\psi}_{3, i_{1} i_{2}}^{(3)} \boldsymbol{\psi}_{4, i_{1} i_{2}}^{(3)} ; \delta \boldsymbol{\alpha}\right)=\sum_{i_{3}=1}^{T P} \frac{\partial \hat{P}^{(3)}\left(\boldsymbol{\alpha}, \boldsymbol{\varphi}_{i_{1}}^{(3)} ; \boldsymbol{\psi}_{1, i_{1} i_{2}}^{(3)} \boldsymbol{\psi}_{2, i_{1} i_{2}}^{(3)}, \boldsymbol{\psi}_{3, i_{1} i_{2}}^{(3)} \boldsymbol{\psi}_{4, i_{1} i_{2}}^{(3)}\right)}{\partial \alpha_{i_{3}}} \delta \alpha_{i_{3}} \text {. }
$$

Using the results shown in Equations (135)-(137) and (159)-(161) in Equation (134) enables the 3rd-order G-differential, $\delta^{(3)} R\left(\mathbf{e}^{0} ; \mathbf{h}_{1}, \mathbf{h}_{2}, \mathbf{h}_{3}\right)$, of the response $R(\mathbf{e})$ at $\mathbf{e}^{0}=$ $\left(\boldsymbol{\alpha}^{0}, \mathbf{u}^{0}\right) \in \mathrm{E}=\mathrm{E}_{\alpha} \times \mathrm{E}_{u}$ in a neighborhood $\left(\mathbf{e}^{0}+\varepsilon \mathbf{h}\right)$ around $\mathbf{e}^{0}=\left(\boldsymbol{\alpha}^{0}, \mathbf{u}^{0}\right) \in \mathrm{E}$ to be expressed as follows:

$$
\delta^{(3)} R\left(\mathbf{e}^{0} ; \mathbf{h}_{1}, \mathbf{h}_{2}, \mathbf{h}_{3}\right)=\sum_{i_{1}=1}^{T P} \sum_{i_{2}=1}^{T P} \sum_{i_{3}=1}^{T P}\left\{R_{i_{1} i_{2} i_{3}}^{(3)}\left(\boldsymbol{\alpha}, \boldsymbol{\varphi}_{i_{1}}^{(3)} ; \boldsymbol{\psi}_{1, i_{1} i_{2}}^{(3)}, \boldsymbol{\psi}_{2, i_{1} i_{2}}^{(3)}, \boldsymbol{\psi}_{3, i_{1} i_{2}}^{(3)}, \boldsymbol{\psi}_{4, i_{1} i_{2}}^{(3)}\right)\right\}_{\boldsymbol{\alpha}^{0}} \delta \alpha_{i_{1}} \delta \alpha_{i_{2}} \delta \alpha_{i_{3}} .
$$

In Equation (162), the quantities $R_{i_{1} i_{2} i_{3}}^{(3)}$ denote the 3rd-order mixed partial sensitivities (Gderivatives) of the response to the model parameters, and are given by the following expression:

$$
\begin{aligned}
& \text { For } i_{1}, i_{2}, i_{3}=1, \ldots, T P: \quad R_{i_{1} i_{2} i_{3}}^{(3)}\left(\boldsymbol{\alpha}, \boldsymbol{\varphi}_{i_{1}}^{(3)} ; \boldsymbol{\psi}_{1, i_{1} i_{2}}^{(3)}, \boldsymbol{\psi}_{2, i_{1} i_{2}}^{(3)}, \boldsymbol{\psi}_{3, i_{1} i_{2}}^{(3)}, \boldsymbol{\psi}_{4, i_{1} i_{2}}^{(3)}\right) \triangleq \\
& \frac{\partial}{\partial \alpha_{i_{3}}}\left[R_{i_{1} i_{2}}^{(2)}\left(\boldsymbol{\alpha}, \boldsymbol{\varphi}_{i_{1}}^{(3)}\right)-\hat{P}^{(3)}\left(\boldsymbol{\alpha}, \boldsymbol{\varphi}_{i_{1}}^{(3)} ; \boldsymbol{\psi}_{1, i_{1} i_{2}}^{(3)}, \boldsymbol{\psi}_{2, i_{1} i_{2}}^{(3)} \boldsymbol{\psi}_{3, i_{1} i_{2}}^{(3)} \boldsymbol{\psi}_{4, i_{1} i_{2}}^{(3)}\right)\right] \\
& +\int_{\Omega_{x}}\left\{\boldsymbol{\psi}_{1, i_{1} i_{2}}^{(3)} \cdot\left[\frac{\partial\left[-\mathbf{A}_{11}^{(1)}(\boldsymbol{\alpha}, \mathbf{u}) \boldsymbol{\psi}_{2, i_{1}}^{(2)}-\mathbf{A}_{21}^{(2)}(\boldsymbol{\alpha}, \mathbf{u}) \boldsymbol{\psi}_{1, i_{1}}^{(2)}+\boldsymbol{\Theta}_{2, i_{1}}^{(2)}\left(\boldsymbol{\alpha}, \mathbf{u} ; \boldsymbol{\psi}^{(1)}\right)\right]}{\partial \alpha_{i_{3}}}\right]\right. \\
& +\boldsymbol{\psi}_{2, i_{1} i_{2}}^{(3)} \cdot\left[\frac{\partial\left[-\mathbf{F}_{11}^{(1)}(\boldsymbol{\alpha}, \mathbf{u}) \boldsymbol{\psi}_{1, i_{1}}^{(2)}+\boldsymbol{\Theta}_{1, i_{1}}^{(2)}\left(\boldsymbol{\alpha}, \mathbf{u} ; \boldsymbol{\psi}^{(1)}\right)\right]}{\partial \alpha_{i_{3}}}\right] \\
& \left.+\boldsymbol{\psi}_{3, i_{1} i_{2}}^{(3)} \cdot\left[\frac{\partial\left[\Theta^{(1)}(\boldsymbol{\alpha}, \mathbf{u})-\mathbf{A}_{11}^{(1)}(\boldsymbol{\alpha}, \mathbf{u}) \boldsymbol{\Psi}^{(1)}\right]}{\partial \alpha_{i_{3}}}\right]+\boldsymbol{\psi}_{4, i_{1} i_{2}}^{(3)} \cdot\left[\frac{\partial[\mathbf{Q}(\boldsymbol{\alpha})-\mathbf{N}(\boldsymbol{\alpha}, \mathbf{u})]}{\partial \alpha_{i_{3}}}\right]\right\} d \mathbf{x} .
\end{aligned}
$$

The 3rd-Level Adjoint Sensitivity System (3rd-LASS), represented by Equation (157) together with the 3rd-level adjoint boundary conditions represented by Equation (150), is independent of parameter variations $\delta \boldsymbol{\alpha}$, as well as of the variations $\delta \mathbf{u}, \delta \boldsymbol{\psi}^{(1)}$, and $\delta \boldsymbol{\psi}^{(2)}$. Solving the 3rd-LASS amounts to solving four equations of the same size as the 1st-LASS, involving two inversions of the forward operator $\mathbf{F}_{11}^{(1)}(\boldsymbol{\alpha}, \mathbf{u})$ and two inversions of the adjoint operator $\mathbf{A}_{11}^{(1)}(\boldsymbol{\alpha}, \mathbf{u})$. These operators are the same as those that also need to be inverted when solving the 2nd-LASS but have different source terms on their respective right-sides. Hence, the same computer program and "solvers" that are used for solving the 1st-LASS and 2nd-LASS can also be used for solving the 3rd-LASS. The 3rd-LASS does not involve any 2nd- or 3rd-order G-derivatives of the operators underlying the original system but involves only the solution/inversion of the same operators that needed to be inverted for solving the 1st-LASS and 2nd-LASS; it is for this reason that this system is called "the 3rd-Level" rather than the "3rd-order" Adjoint Sensitivity System.

For each 2nd-order mixed differential $\delta R_{i_{1} i_{2}}^{(2)}$, solving the 3rd-LASS once provide the complete set of all of the corresponding 3rd-order sensitivities. It is also important to note that by solving the 3rd-LASS TP-times, the 3rd-order mixed sensitivities will be computed twice, in two different ways, thereby providing an intrinsic (numerical) verification that 
the 3rd-Level adjoint functions $\boldsymbol{\psi}_{i_{1} i_{2}}^{(3)} \triangleq\left(\boldsymbol{\psi}_{1, i_{1} i_{2}}^{(3)} \boldsymbol{\psi}_{2, i_{1} i_{2}}^{(3)} \boldsymbol{\psi}_{3, i_{1} i_{2}}^{(3)}, \psi_{4, i_{1} i_{2}}^{(3)}\right) \in \mathrm{H}_{\psi}^{(3)}\left(\Omega_{x}\right)$ are computed accurately. Since each of the G-differentials $\delta R_{i_{1} i_{2}}^{(2)}\left(\boldsymbol{\alpha}, \boldsymbol{\varphi}_{i_{1}}^{(3)} ; \delta \boldsymbol{\alpha}, \delta \mathbf{u}, \delta \boldsymbol{\psi}^{(1)}, \delta \boldsymbol{\psi}_{1, i_{1}}^{(2)} \delta \boldsymbol{\Psi}_{i, i_{1}}^{(2)}\right)$ contains one complete row $\left(i_{3}=1, \ldots, T P\right)$ of the mixed second-order partial sensitivities, $R_{i_{1} i_{2} i_{3}}^{(3)}\left(\boldsymbol{\alpha}, \mathbf{u} ; \boldsymbol{\varphi}_{i_{1}}^{(3)} ; \boldsymbol{\psi}_{1, i_{1} i_{2}}^{(3)} \boldsymbol{\psi}_{2, i_{1} i_{2}}^{(3)}, \boldsymbol{\psi}_{3, i_{1} i_{2}}^{(3)}, \boldsymbol{\psi}_{4, i_{1} i_{2}}^{(3)}\right)$, the exact computation of all of the partial 3rd-order sensitivities, $\left\{\partial^{3} R(\boldsymbol{\alpha}, \mathbf{u}) / \partial \alpha_{i_{1}} \partial \alpha_{i_{2}} \partial \alpha_{i_{3}}\right\}_{\left(\boldsymbol{\alpha}^{0}, \mathbf{u}^{0}\right)}$, requires at most $O\left(T P^{2}\right)$ largescale (adjoint) computations, as opposed to $O\left(T P^{3}\right)$ large-scale computations as would be required by forward methods. The 3rd-LASS also provides flexibility for prioritizing the computation of the 3rd-order sensitivities, just like the 2nd-LASS provided for the selective computation of the 2nd-order partial sensitivities of the response $R(\mathbf{e})$. Thus, the computation of the 3rd-order sensitivities should be performed in an order of priorities based on the ranking of the relative magnitudes of the 2nd-order sensitivities: the mixed 3rd-order sensitivities corresponding to the largest relative 2nd-order sensitivity should be computed first; then the computation of the 3rd-order sensitivities that correspond to the second largest relative 2nd-order response sensitivity should be considered next, and so on.

To implement the 3rd-CASAM-N into a computer code, the following equations would need to be programmed and solved numerically: (i) the 3rd-LASS represented by Equation (157) together with the corresponding initial/boundary conditions represented by Equation (150); (ii) the expresions provided in Equation (163) for computing the 3rd-order sensitivities, which involves user-chosen quadrature-formulas in order to perform the integrations over the various quantities in the respective integrands; these quantities would need to be evaluated at the chosen quadrature-points/nodes, of course.

\section{Illustrative Comparison of CPU-Times for a Large-Scale OECD/NEA Reactor Physics Benchmark: 3rd-CASAM Versus Finite Differences}

In previous works [36-38] the 3rd-Order Comprehensive Adjoint Sensitivity Analysis of Response-Coupled Linear Forward/Adjoint Systems" (3rd-CASAM-L) developed by Cacuci [34] was applied to perform a 3rd-order sensitivity and uncertainty analysis of an OECD/NEA reactor physics benchmark [32]. The response of interest for this benchmark was the leakage of particles out of the benchmark, which had been experimentally measured. This benchmark was modeled by using the PARTISN [33] code to solve numerically the Boltzmann neutron transport equation. The computational model comprised 21,976 uncertain model parameters, as follows: 180 group-averaged total microscopic cross sections; 21,600 group-averaged scattering microscopic cross sections; 120 fission process parameters; 60 fission spectrum parameters; 10 source parameters; and 6 isotopic number densities. After having computed the 21,976 first-order sensitivities and 482,944,576 second-order sensitivities of the benchmark's leakage with respect to all model parameters of the PERP benchmark, it was found [26-31] that the most important parameters, to which the response exhibited the largest sensitivities, were the total microscopic cross sections. Ranked second in decreasing order of magnitudes were the sensitivities of the leakage response to the isotopic number densities, followed by the sensitivities the average number of neutrons per fission. Significantly smaller were the sensitivities to the fission cross sections and the sensitivities to the source parameters, while the sensitivities to the scattering cross sections were negligible.

In particular, it was shown $[26,31]$ for the PERP benchmark that neglecting second (and higher) order sensitivities would cause the expectation of the response to differ by $40 \%$ from its computed value for $5 \%$ relative standard deviations for uncorrelated total cross sections. Subsequent investigations [36-38] showed that for a 5\% uniform standard deviation, the 1st-order sensitivities contributed $8 \%$ to the response variance; the 2 ndorder sensitivities contributed $17 \%$ to the response variance; the 3rd-order sensitivities contributed $75 \%$ to the response variance. These effects were rapidly amplified as the uncertainties in the cross sections increased. Neglecting the contributions stemming from the third-order sensitivities would cause a large non-conservative errors. 
The results for the OECD/NEA benchmark were obtained [26-31,36-38] by using a DELL computer (AMD FX-8350) with an 8-core processor, the CPU-time for a typical adjoint computation was ca. $24 \mathrm{~s}$, and the CPU-time for computing the integrals over the various adjoint functions which appear in the definition of the respective sensitivities ca. 0.004 seconds. The CPU-time for a typical forward computation was ca. $45 \mathrm{~s}$. The computational times needed for obtaining all of the distinct 1st-, 2nd-, and 3rd-order sensitivities of the PERP leakage response with respect to the 180 microscopic total cross sections using the 4th-CASAM were as follows [36-38]:

(i) To compute the 180 first-order sensitivities, 1 adjoint computation is needed in order to obtain the $1^{\text {st }}$-level adjoint function. Thus, the CPU-time needed is ca. $24 \mathrm{~s}$ plus ca. $1 \mathrm{~s}$ for computing the 180 integrals over this adjoint function. By comparison, ca. $270 \mathrm{~min}$ are needed to compute these 180 1st-order sensitivities using a finitedifference (FD-)formula.

(ii) To compute the $180(180+1) / 2=16,290$ distinct 2 nd-second-order sensitivities, $180 \times 2=360$ adjoint computations are needed to obtain the 2nd-level adjoint functions, requiring ca. $2.4 \mathrm{~h}$ CPU-time; ca. 3 additional minutes were required for computing the integrals over these adjoint functions. By comparison, ca. $810 \mathrm{~h}$ are needed to compute these 2nd-order sensitivities using FD-formula.

(iii) To compute the $180(180+1)(180+2) / 3 !=988,260$ distinct $3 r d$-third-order sensitivities, 32,940 adjoint computations are needed to obtain the 3rd-level adjoint functions, requiring ca. $220 \mathrm{~h}$ CPU-time; an additional $0.6 \mathrm{~h}$ were needed for computing the integrals over these adjoint functions. By comparison, ca. 98,817 $\mathrm{h}$ would have been needed to compute these 3rd-order sensitivities using FD-formula; this CPU-estimate has been obtained [36-38] by rounding up minutes and seconds to the nearest hour. The reason for providing these "best-estimates" of CPU-times (rounded to the nearest CPU-hour) is to enable scaling comparisons between the DELL AMD FX-8350 (with an 8-core processor) computer and some other (more/less performant) computer system.

\section{Concluding Discussion}

This work reviewed the state-of-the-art methodologies for the deterministic sensitivity analysis of nonlinear systems and deterministic quantification of uncertainties induced in model responses by uncertainties in the model parameters. The need for computing highorder sensitivities was underscored by presenting an analytically solvable model of neutron scattering in a hydrogenous medium for which all of the response's relative sensitivities had the same absolute value of unity. It was shown that the wider the distribution of model parameters, the higher the order of sensitivities needed to achieve a desired level of accuracy in representing the response and in computing the response's expectation, variance, skewness and kurtosis.

This work also presented new mathematical expressions that extended to the sixth order of the current state-of-the-art fourth-order formulas for computing the fourth-order correlations among the computed model responses and model parameters. Another novelty presented in this work was the mathematical framework of the 3rd-Order Comprehensive Adjoint Sensitivity Analysis Methodology for Nonlinear Systems (3rd-CASAM-N), which enabled the most efficient computation of the exact expressions of the 1st-, 2nd- and 3rdorder functional derivatives ("sensitivities") of a model's response to the underlying model parameters, including the imprecisely known initial, boundary and/or interface conditions. The 3rd-CASAM-N is the nonlinear counterpart of the 3rd-Order Comprehensive Adjoint Sensitivity Analysis Methodology for Response-Coupled Forward and Adjoint Linear Systems (4th-CASAM-L) conceived by Cacuci [34], which enables the efficient computation of the exact expressions of the 3rd-order sensitivities of a model response that depends on both the forward and adjoint state functions, with respect to all of the parameters underlying the respective forward and adjoint systems. Notably, the computations of the 2nd- and 3rd-level adjoint functions use the same forward and adjoint solvers as used for solving the original forward and adjoint systems. 
The CPU times needed for computing 1st-, 2nd- and 3rd-order response sensitivities to the model parameters for an OECD/NEA reactor physics benchmark [32] using the 3rd-CASAM were compared to the corresponding CPU times that would be required if the respective sensitivities were computed by finite-difference methods. These comparisons highlighted the fact that the finite-difference schemes would not only provide approximate values for the respective sensitivities (in contradistinction to the 3rd-CASAM-N/L, which provides exact expressions for the sensitivities) but would simply be unfeasible for computing sensitivities of order higher than first order. Since statistical methods require orders of magnitude, more computational time and resources than finite-differences, it is evident that the statistical methods could not even compute the first-order sensitivities of the OECD/NEA reactor physics benchmark's response to the benchmark's 21,976 model parameters.

The 3rd-CASAM-N presented in this work provides a fundamentally important step in the quest to overcome the "curse of dimensionality" in the sensitivity analysis, uncertainty quantification and predictive modeling. Ongoing work will generalize the 3rd-CASAM-N to enable the exact computation of arbitrarily higher order sensitivities of responses to model parameters, both for linear and nonlinear systems, while overcoming the curse of dimensionality.

Funding: This research received no external funding.

Institutional Review Board Statement: Not applicable.

Informed Consent Statement: Not applicable.

Data Availability Statement: Not applicable.

Conflicts of Interest: The author declares no conflict of interest.

\section{Appendix A}

The model and boundary parameters are imprecisely known (uncertain) quantities. Their true values (which are unknown) will differ from their nominal (or mean) values (which are known) by quantities denoted as $\delta \boldsymbol{\alpha} \triangleq\left(\delta \alpha_{1}, \ldots, \delta \alpha_{T P}\right)$, where $\delta \alpha_{i} \triangleq \alpha_{i}-\alpha_{i}^{0}$. Since the forward state functions $\mathbf{u}(\mathbf{x})$ are related to the model and boundary parameters $\alpha$ through Equations (1) and (2), it follows that the variations $\delta \alpha$ in the model and boundary parameter will cause corresponding variations $\delta \mathbf{u}(\mathbf{x}) \triangleq\left[\delta u_{1}(\mathbf{x}), \ldots, \delta u_{T I}(\mathbf{x})\right]^{\dagger}$ around the nominal solution $\mathbf{u}^{0}(\mathbf{x})$ in the forward state functions. In turn, the variations $\delta \boldsymbol{\alpha}$ and $\delta \mathbf{u}(\mathbf{x})$ will induce variations in the system's response. For the mathematical derivations to follow, it is convenient to introduce the vector $\mathbf{e} \triangleq(\mathbf{u}, \boldsymbol{\alpha})$, which is defined on the product of the vector fields of the model parameters and state functions. The nominal value of $\mathbf{e} \triangleq(\mathbf{u}, \boldsymbol{\alpha})$ is denoted as $\mathbf{e}^{0} \triangleq\left(\mathbf{u}^{0}, \boldsymbol{\alpha}^{0}\right)$.

Appendix A.1. The First-Order Comprehensive Adjoint Sensitivity Analysis Methodology for Nonlinear Systems (1st-CASAM-N)

The most general definition of the 1st-order total sensitivity of the operator-valued model response $R(\mathbf{u}, \boldsymbol{\alpha})$, as defined in Equation (5), to variations $\delta \mathbf{u}(\mathbf{x})$ and $\delta \boldsymbol{\alpha}$ around the nominal values $\mathbf{u}^{0}$ and $\alpha^{0}$, respectively, has been provided in the pioneering works of Cacuci $(1981 \mathrm{a}, \mathrm{b})$ in terms of the first-order Gateaux-variation $\delta R\left(\mathbf{u}^{0}, \boldsymbol{\alpha}^{0} ; \delta \mathbf{u}, \delta \boldsymbol{\alpha}\right)$ of $R(\mathbf{u}, \boldsymbol{\alpha})$, which is defined as follows:

$$
\delta R\left(\mathbf{e}^{0} ; \mathbf{h}\right) \triangleq\left\{\frac{d}{d \varepsilon} \prod_{i=1}^{T I} \int_{\lambda_{i}\left(\boldsymbol{\alpha}^{0}+\varepsilon \delta \boldsymbol{\alpha}\right)}^{\omega_{i}\left(\boldsymbol{\alpha}^{0}+\varepsilon \delta \boldsymbol{\alpha}\right)} S\left(\mathbf{u}^{0}+\varepsilon \delta \mathbf{u}, \boldsymbol{\alpha}^{0}+\varepsilon \delta \boldsymbol{\alpha} ; \mathbf{x}\right) d x_{i}\right\}_{\varepsilon=0},
$$

for a scalar quantity $\varepsilon$ and arbitrary vector $\mathbf{h}(\mathbf{x}) \triangleq[\delta \mathbf{u}(\mathbf{x}) ; \delta \boldsymbol{\alpha}]^{\dagger}$ in a neighborhood $\left(\mathbf{e}^{0}+\varepsilon \mathbf{h}\right)$ around $\mathbf{e}^{0}$. The G-differential $\delta R\left(\mathbf{e}^{0} ; \mathbf{h}\right)$ is an operator defined on the same domain as 
$R(\mathbf{e})$, has the same range as $R(\mathbf{e})$, and provides the total 1st-order total sensitivity of $R(\mathbf{e})$ with respect to variations in the model's parameters and state functions. The Gdifferential $\delta R\left(\mathbf{e}^{0} ; \mathbf{h}\right)$ satisfies the relation: $R\left(\mathbf{e}^{0}+\varepsilon \mathbf{h}\right)-R\left(\mathbf{e}^{0}\right)=\delta R\left(\mathbf{e}^{0} ; \mathbf{h}\right)+\Delta(\mathbf{h})$, with $\lim _{\varepsilon \rightarrow 0}[\Delta(\varepsilon \mathbf{h})] / \varepsilon=0$. The existence of the G-variation $\delta R\left(\mathbf{e}^{\mathbf{0}} ; \mathbf{h}\right)$ does not guarantee its numerical computability. Numerical methods most often require that $\delta R\left(\mathbf{e}^{0} ; \mathbf{h}\right)$ be linear in $\mathbf{h} \triangleq(\delta \mathbf{u} ; \delta \boldsymbol{\alpha})$ in a neighborhood $\left(\mathbf{e}^{0}+\varepsilon \mathbf{h}\right)$ around $\mathbf{e}^{0}$. Formally, the necessary and sufficient conditions for the G-variation $\delta R\left(\mathbf{e}^{0} ; \mathbf{h}\right)$ of a nonlinear operator $R(\mathbf{e})$ to be linear and continuous in $\mathbf{h}$ in a neighborhood $\left(\mathbf{e}^{0}+\varepsilon \mathbf{h}\right)$ around $\mathbf{e}^{0}=\left(\boldsymbol{\alpha}^{0}, \mathbf{u}^{0}\right)$, and thus admit a total first-order G-derivative, are as follows:

(i) $\quad R(\mathbf{e})$ must satisfy a weak Lipschitz condition at $\mathbf{e}^{0}$, i.e.,

$$
\left\|R\left(\mathbf{e}^{0}+\varepsilon \mathbf{h}\right)-R\left(\mathbf{e}^{0}\right)\right\| \leq k\left\|\varepsilon \mathbf{e}^{0}\right\|, \quad k<\infty
$$

(ii) $\quad R(\mathbf{e})$ must satisfy the following condition for two arbitrary vectors $\mathbf{h}_{1}$ and $\mathbf{h}_{2}$ defined in the same vector space as $\mathbf{e}^{0}$ :

$$
R\left(\mathbf{e}^{0}+\varepsilon \mathbf{h}_{1}+\varepsilon \mathbf{h}_{2}\right)-R\left(\mathbf{e}^{0}+\varepsilon \mathbf{h}_{1}\right)-R\left(\mathbf{e}^{0}+\varepsilon \mathbf{h}_{2}\right)+R\left(\mathbf{e}^{0}\right)=o(\varepsilon) .
$$

In practice, it is usually observed directly in the right-side of Equation (A1) is linear in $\delta \mathbf{u}(\mathbf{x})$. Numerical methods (e.g., Newton's method and variants thereof) for solving Equations (1) and (2) also require the existence of the 1st-order G-derivatives of original model equations, in which case the components of the operators which appear in these equations must also satisfy the conditions described in Equations (A2) and (A3).

To proceed, the conditions described in Equations (A2) and (A3) will henceforth be considered to be satisfied by the operators underlying the physical system, in which case the partial G-derivatives of $R(\mathbf{e})$ at $\mathbf{e}^{0}$ with respect to $\mathbf{u}(\mathbf{x})$ and $\alpha$ exist. It follows that that the first-order G-variation $\delta R\left(\mathbf{e}^{0} ; \mathbf{h}\right)$ defined in Equation (A4) can be written in the following form:

$$
\delta R\left(\mathbf{e}^{0} ; \mathbf{h}\right)=\left\{\delta R\left(\mathbf{e}^{0} ; \mathbf{h}\right)\right\}_{d i r}+\left\{\delta R\left(\mathbf{e}^{0} ; \mathbf{h}\right)\right\}_{i n d^{\prime}}
$$

where the "direct-effect" term $\left\{\delta R\left(\mathbf{e}^{0} ; \mathbf{h}\right)\right\}_{\text {dir }}$ is defined as follows:

$$
\begin{aligned}
& \left\{\delta R\left(\mathbf{e}^{0} ; \mathbf{h}\right)\right\}_{d i r} \triangleq \prod_{i=1}^{T I} \int_{\lambda_{i}\left(\boldsymbol{\alpha}^{0}\right)}^{\omega_{i}\left(\boldsymbol{\alpha}^{0}\right)} d x_{i}\left\{\frac{\partial S(\mathbf{e} ; \boldsymbol{\alpha})}{\partial \boldsymbol{\alpha}}\right\}_{\left(\mathbf{e}^{0}\right)} \delta \boldsymbol{\alpha} \\
& +\sum_{j=1}^{T I} \prod_{\substack{i=1 \\
i \neq j}}^{\omega_{i}\left(\boldsymbol{\lambda}_{i}\left(\boldsymbol{\alpha}^{0}\right)\right.} d x_{i}\left\{S\left[\mathbf{u}\left(x_{1}, ., \omega_{i}(\boldsymbol{\alpha}), ., x_{N_{x}}\right) ; \boldsymbol{\alpha}\right] \frac{\partial \omega_{i}(\boldsymbol{\alpha})}{\partial \boldsymbol{\alpha}} \delta \boldsymbol{\alpha}\right\}_{\left(\mathbf{e}^{0}\right)} \\
& -\sum_{j=1}^{T I} \prod_{\substack{i=1 \\
i \neq j}}^{\omega_{i} \int_{i}\left(\boldsymbol{\alpha}^{0}\right)} d x_{i}\left\{S\left[\mathbf{u}\left(x_{1}, ., \lambda_{i}(\boldsymbol{\alpha}), ., x_{N_{x}}\right) ; \boldsymbol{\alpha}\right] \frac{\partial \lambda_{i}(\boldsymbol{\alpha})}{\partial \boldsymbol{\alpha}} \delta \boldsymbol{\alpha}\right\}_{\left(\mathbf{e}^{0}\right)} .
\end{aligned}
$$

and where the "indirect-effect" term $\left\{\delta R\left(\mathbf{e}^{0} ; \mathbf{h}\right)\right\}_{\text {ind }}$ is defined as follows:

$$
\left\{\delta R\left(\mathbf{e}^{0} ; \mathbf{h}\right)\right\}_{i n d} \triangleq \prod_{i=1}^{T I} \int_{\lambda_{i}\left(\boldsymbol{\alpha}^{0}\right)}^{\omega_{i}\left(\boldsymbol{\alpha}^{0}\right)} d x_{i}\left\{\frac{\partial S(\mathbf{e} ; \mathbf{x})}{\partial \mathbf{u}}\right\}_{\left(\mathbf{e}^{0}\right)} \delta \mathbf{u}(\mathbf{x}) .
$$

The quantity $\left\{\delta R\left(\mathbf{e}^{0} ; \mathbf{h}\right)\right\}_{\text {dir }}$ in Equation (A5) is called the "direct effect term" because it arises directly from parameter variations. The direct-effect term can be computed once the base-case values $\mathbf{e}^{0}$ are available. On the other hand, the "indirect effect term" $\left\{\delta R\left(\mathbf{e}^{0} ; \mathbf{h}\right)\right\}_{\text {ind }}$ 
defined in Equation (A6) arises indirectly, through the variations in the state functions which are caused, through the model, by parameter variations. The indirect-effect term can be quantified only after having determined the variations $\delta \mathbf{u}(\mathbf{x})$ in terms of the variations $\delta \alpha$. The first-order relationship between the variations $\delta \mathbf{u}(\mathbf{x})$ and $\delta \alpha$ is determined by taking the G-differentials of Equations (1) and (2). Thus, applying the definition of the G-differential to Equations (1) and (2) yields the following equations:

$$
\begin{gathered}
\left\{\mathbf{F}_{11}^{(1)}(\boldsymbol{\alpha}, \mathbf{u})\right\}_{\left(\mathbf{e}^{0}\right)} \delta \mathbf{u}=\left\{\mathbf{Q}^{(1)}(\boldsymbol{\alpha}, \mathbf{u} ; \delta \boldsymbol{\alpha})\right\}_{\left(\mathbf{e}^{0}\right)^{\prime}}, \mathbf{x} \in \Omega_{x}, \\
\left\{\mathbf{B}_{F}^{(1)}(\boldsymbol{\alpha}, \mathbf{u} ; \delta \boldsymbol{\alpha}, \delta \mathbf{u})\right\}_{\left(\mathbf{e}^{0}\right)}=0, \mathbf{x} \in \partial \Omega_{x}\left(\boldsymbol{\alpha}^{0}\right),
\end{gathered}
$$

where the superscript "(1)" indicates "1st-Level," the letter " $\mathrm{F}$ " (used as "operator" and "subscript") indicates "Forward", the letter "B" indicates "boundary and/or initial conditions," and where the following definitions were used:

$$
\begin{aligned}
& \mathbf{F}_{11}^{(1)}(\boldsymbol{\alpha}, \mathbf{u}) \triangleq\left\{\frac{\partial \mathbf{N}(\boldsymbol{\alpha}, \mathbf{u})}{\partial \mathbf{u}}\right\} ; \quad \mathbf{Q}^{(1)}(\boldsymbol{\alpha}, \mathbf{u} ; \delta \boldsymbol{\alpha}) \triangleq \frac{\partial[\mathbf{Q}(\boldsymbol{\alpha})-\mathbf{N}(\boldsymbol{\alpha}, \mathbf{u})]}{\partial \boldsymbol{\alpha}} \delta \boldsymbol{\alpha} ; \\
& \mathbf{B}_{F}^{(1)}(\boldsymbol{\alpha}, \mathbf{u} ; \delta \boldsymbol{\alpha}, \delta \mathbf{u}) \triangleq \frac{\partial \mathbf{B}(\boldsymbol{\alpha}, \mathbf{u})}{\partial \mathbf{u}} \delta \mathbf{u}+\frac{\partial[\mathbf{C}(\boldsymbol{\alpha})-\mathbf{B}(\boldsymbol{\alpha}, \mathbf{u})]}{\partial \boldsymbol{\alpha}} \delta \boldsymbol{\alpha} \\
& +\left\{\sum_{i=1}^{T I}\left[\frac{\partial \mathbf{B}[\mathbf{u}(\mathbf{x}) ; \boldsymbol{\alpha}]}{\partial \omega_{i}} \frac{\partial \omega_{i}(\boldsymbol{\alpha})}{\partial \boldsymbol{\alpha}} \delta \boldsymbol{\alpha}\right]_{\mathbf{x}=\mathbf{\omega}}+\left[\frac{\partial \mathbf{B}[\mathbf{u}(\mathbf{x}) ; \boldsymbol{\alpha}]}{\partial \lambda_{i}} \frac{\partial \lambda_{i}(\boldsymbol{\alpha})}{\partial \boldsymbol{\alpha}} \delta \boldsymbol{\alpha}\right]_{\mathbf{x}=\lambda}\right\}_{\left(\mathbf{e}^{0}\right)} .
\end{aligned}
$$

The partial G-derivatives $\partial \mathbf{N}(\boldsymbol{\alpha}, \mathbf{u}) / \partial \mathbf{u}$ and $\partial \mathbf{N}(\boldsymbol{\alpha}, \mathbf{u}) / \partial \boldsymbol{\alpha}$ appearing in Equation (A9) are matrices of the form

$$
\frac{\partial \mathbf{N}(\boldsymbol{\alpha}, \mathbf{u})}{\partial \mathbf{u}} \triangleq\left(\begin{array}{ccc}
\frac{\partial N_{1}}{\partial u_{1}} & \cdots & \frac{\partial N_{1}}{\partial u_{T D}} \\
\vdots & \ddots & \vdots \\
\frac{\partial N_{T D}}{\partial u_{1}} & \cdots & \frac{\partial N_{T D}}{\partial u_{T D}}
\end{array}\right) ; \delta \mathbf{u}(\mathbf{x}) \triangleq\left(\begin{array}{c}
u_{1}(\mathbf{x}) \\
\vdots \\
u_{T D}(\mathbf{x})
\end{array}\right)^{\dagger} ; \frac{\partial \mathbf{N}(\boldsymbol{\alpha}, \mathbf{u})}{\partial \boldsymbol{\alpha}} \triangleq\left(\begin{array}{ccc}
\frac{\partial N_{1}}{\partial \alpha_{1}} & \cdots & \frac{\partial N_{1}}{\partial \alpha_{T P}} \\
\vdots & \ddots & \vdots \\
\frac{\partial N_{T D}}{\partial \alpha_{1}} & \cdots & \frac{\partial N_{T D}}{\partial \alpha_{T P}}
\end{array}\right)
$$

The other partial G-derivatives that appear in Equations (A9) and (A10) are also matrices having structures similar to those defined in Equation (A11). The system comprising Equations (A7) and (A8) are called the "1st-Level Variational Sensitivity System" (1st-LVSS). For a given vector of parameter variations $\delta \alpha$ in a neighborhood around $\alpha^{0}$, the 1st-LVSS could be solved to obtain $\delta \mathbf{u}$ as a function of $\delta \boldsymbol{\alpha}$. In turn, the relationship between $\delta \mathbf{u}$ and $\delta \alpha$ would be used to eliminate the appearance of $\delta \mathbf{u}$ in Equation (A4); consequently, Equation (A4) could be expressed in the form

$$
\delta R\left(\boldsymbol{\alpha}^{0}, \mathbf{u}^{0} ; \delta \boldsymbol{\alpha}\right)=\sum_{i_{1}=1}^{N_{\alpha}} R_{i_{1}}^{(1)} \delta \alpha_{i_{1}}
$$

where the quantities $R_{i_{1}}^{(1)}$ are independent of the parameter variations $\delta \alpha_{i_{1}}$ (although they may depend on $\boldsymbol{\alpha}^{0}, \mathbf{u}^{0}$, and/or other known quantities) and represent the 1st-order partial sensitivities (1st-order partial G-derivatives) of the response $R(\mathbf{e})$ with respect to each of the model parameters $\alpha_{i}$, evaluated at the nominal values $\mathbf{e}^{0}$. The partial sensitivities $R_{i_{1}}^{(1)}$ of the response $R(\mathbf{e})$ with respect to each of the model parameters $\delta \alpha_{i_{1}}$, evaluated at the nominal values $\mathbf{e}^{0}=$ $\left(\boldsymbol{\alpha}^{0}, \mathbf{u}^{0}\right)$, are obtained by successively setting $\left(\delta \alpha_{i}=1, \delta \alpha_{j}=0\right.$ for $\left.j \neq i, i=1, \ldots, N_{\alpha}\right)$ in the expression of $\delta R\left(\mathbf{e}^{0} ; \mathbf{h}\right)$ given in Equation (A4). Computing the (total) response sensitivity $\delta R\left(\mathbf{e}^{0} ; \mathbf{h}\right)$ by using the ( $\delta \boldsymbol{\alpha}$-dependent) solution $\delta \mathbf{u}$ of the 1 st-LVSS is called $[2,3,50]$ the Forward Sensitivity Analysis Procedure (FSAP). From the standpoint of computational costs and effort, the FSAP requires require $O(T P)$ large-scale forward computations; therefore the FSAP is advantageous to employ only if, in the problem under consideration, the number $T R$ of "total number of model responses" of interest exceeds the number of system parameters and/or parameter variations of interest. This is rarely the case in practice, however, since 
most problems of practical interest are characterized by many parameters (i.e., $\alpha$ has many components) and comparatively few responses. In such situations, it is not economical to employ the FSAP since it becomes prohibitively expensive to solve repeatedly the $\delta \alpha$ dependent 1st-LVSS in order to determine As explained in the book by Cacuci [25], even though the 1st-LVSS contains first-order parameter and state-functions variations, it is called "first-level" (rather than "first-order") in anticipation of determining second-order sensitivities, which will use "second-level" forward and adjoint systems. These "second-level" systems will not be called "second-order" because they will not contain second-order parameter and/or state-function variations, but will also contain only first-order variations, even though they will be used for determining second-order sensitivities. Similar terminology, i.e., "third-level" (as opposed to "third-order") forward/adjoint systems, will be used for determining the third-order sensitivities.

In most practical situations, the number of model parameters exceeds significantly the number of functional responses of interest, i.e., $T R \ll T P$. In such cases, the Adjoint Sensitivity Analysis Procedure (ASAP) conceived by Cacuci $[2,3,50]$ is the most efficient method for computing exactly the first-order sensitivities since it requires only $O(T R)$ large-scale computations. The implementation of the ASAP requires the introduction of adjoint operators, which can be defined in Banach spaces but are most useful in Hilbert spaces. The spaces $\mathrm{E}_{u}$ and $\mathrm{E}_{Q}$ are henceforth considered to be Hilbert spaces and denoted as $\mathrm{H}_{u}\left(\Omega_{x}\right)$ and $\mathrm{H}_{Q}\left(\Omega_{x}\right)$, respectively. The elements of $\mathrm{H}_{u}\left(\Omega_{x}\right)$ and $\mathrm{H}_{Q}\left(\Omega_{x}\right)$ are, as before, vector-valued functions defined on the open set $\Omega_{x} \subset \mathbb{R}^{J_{x}}$, with smooth boundary $\partial \Omega_{x}$. On $\mathrm{H}_{u}\left(\Omega_{x}\right)$, the inner product of two vectors $\mathbf{u}^{(a)} \in \mathrm{H}_{u}$ and $\mathbf{u}^{(b)} \in \mathrm{H}_{u}$ will be denoted as $\left\langle\mathbf{u}^{(a)}, \mathbf{u}^{(b)}\right\rangle_{u}$. Typically, the inner product $\left\langle\mathbf{u}^{(a)}, \mathbf{u}^{(b)}\right\rangle_{u}$ is defined as $\delta \mathbf{u}$ for all possible variations $\delta \alpha$ in the model parameters.

$$
\left\langle\mathbf{u}^{(a)}, \mathbf{u}^{(b)}\right\rangle_{u} \triangleq \int_{\Omega_{x}} \mathbf{u}^{(a)}(\mathbf{x}) \cdot \mathbf{u}^{(b)}(\mathbf{x}) d \mathbf{x}=\prod_{i=1}^{T I} \int_{\lambda_{i}\left(\boldsymbol{\alpha}^{0}\right)}^{\omega_{i}\left(\boldsymbol{\alpha}^{0}\right)} \mathbf{u}^{(a)}(\mathbf{x}) \cdot \mathbf{u}^{(b)}(\mathbf{x}) d x_{i} .
$$

where the dot indicates the "scalar product of two vectors" defined as follows: $\mathbf{u}^{(a)}(\mathbf{x}) \cdot \mathbf{u}^{(b)}(\mathbf{x}) \triangleq$ $\sum_{i=1}^{T D} u_{i}^{(a)} u_{i}^{(b)}$. It is important to note that the inner product defined in Equation (A16) is continuous in $\alpha$, i.e., it holds at any value particular value of $\alpha$, including at the nominal parameter values $\boldsymbol{\alpha}^{0}$. The inner product on $\mathrm{H}_{Q}\left(\Omega_{x}\right)$ of two vectors $\mathbf{Q}^{(a)} \in \mathrm{H}_{Q}$ and $\mathbf{Q}^{(b)} \in \mathrm{H}_{Q}$ will be denoted as $\left\langle\mathbf{Q}^{(a)}, \mathbf{Q}^{(b)}\right\rangle_{Q^{\prime}}$ and has the same form as shown in Equation (A13). These Hilbert spaces are customarily self-dual.

The goal of the 1st-Order Comprehensive Adjoint Sensitivity Analysis Methodology (1stCASAM) is to compute the "indirect effect term" defined in Equation (A6) exactly and efficiently without needing to compute explicitly the variations $\delta \mathbf{u}$. The fundamental ideas underlying the 1st-CASAM have been conceived by Cacuci $[2,3,50]$ and they aim at eliminating the appearance of the variation $\delta \mathbf{u}$ in the indirect-effect term defined in Equation (A6), by expressing the right-side of Equation (A6) in terms of adjoint functions that are the solutions of the "1st-Level Adjoint Sensitivity System (1st-LASS)." The construction of the 1stLASS commences by requiring that the following relationship hold for an arbitrary vector $\boldsymbol{\psi}^{(1)}(\mathbf{x}) \equiv\left[\psi_{1}^{(1)}(\mathbf{x}), \ldots, \psi_{N_{u}}^{(1)}(\mathbf{x})\right] \in H_{Q}$, where the superscript " $(1)$ " indicates "1st-Level":

$$
\left\langle\boldsymbol{\psi}^{(1)}, \mathbf{F}_{11}^{(1)}\left(\boldsymbol{\alpha}^{0}, \mathbf{u}^{0}\right) \delta \mathbf{u}\right\rangle_{Q}=\left\langle\mathbf{A}_{11}^{(1)}\left(\boldsymbol{\alpha}^{0}, \mathbf{u}^{0}\right) \boldsymbol{\psi}^{(1)}, \delta \mathbf{u}\right\rangle_{u}+\left\{P^{(1)}\left(\boldsymbol{\alpha}^{0}, \mathbf{u}^{0} ; \boldsymbol{\psi}^{(1)} ; \delta \mathbf{u}\right)\right\}_{\partial \Omega_{x}}{ }^{\prime}
$$

where $\mathbf{A}_{11}^{(1)}(\boldsymbol{\alpha}, \mathbf{u}) \triangleq\left[\mathbf{F}_{11}^{(1)}(\boldsymbol{\alpha}, \mathbf{u})\right]^{*}$ denotes the adjoint operator of $\mathbf{F}_{11}^{(1)}(\boldsymbol{\alpha}, \mathbf{u}) \triangleq \partial \mathbf{N}(\boldsymbol{\alpha}, \mathbf{u}) / \partial \mathbf{u}$. In this work, the symbol [ ] will be used to indicate "adjoint." The quantity $\left\{P^{(1)}\left(\boldsymbol{\alpha}, \mathbf{u} ; \boldsymbol{\psi}^{(1)} ; \delta \mathbf{u}\right)\right\}_{\partial \Omega_{x}}$ in Equation (A14) denotes the associated bilinear form evaluated on $\partial \Omega_{x}$. In certain situations, it might be computationally advantageous to include certain 
boundary components of $\left\{P^{(1)}\left(\boldsymbol{\alpha}, \mathbf{u} ; \boldsymbol{\psi}^{(1)} ; \delta \mathbf{u}\right)\right\}_{\partial \Omega_{x}}$ into the components of $\mathbf{A}_{11}^{(1)}(\boldsymbol{\alpha}, \mathbf{u})$. Note that the formal adjoint, $\mathbf{A}_{11}^{(1, f \circ r)}(\boldsymbol{\alpha}, \mathbf{u})$, of $\mathbf{F}_{11}^{(1)}(\boldsymbol{\alpha}, \mathbf{u})$, is defined as the TD $\times$ TD matrix obtained by transposing the formal adjoints of the components of the TD $\times T D$ matrix $\partial \mathbf{N}(\boldsymbol{\alpha}, \mathbf{u}) / \partial \mathbf{u}$ in Equation (A14). The formal adjoint, $\mathbf{A}_{11}^{(1, f \circ r)}(\boldsymbol{\alpha}, \mathbf{u})$, will usually differ from "the adjoint" operator $\mathbf{A}_{11}^{(1)}(\boldsymbol{\alpha}, \mathbf{u}) \triangleq\left[\mathbf{F}_{11}^{(1)}(\boldsymbol{\alpha}, \mathbf{u})\right]^{*}$.

The domain of $\mathbf{A}_{11}^{(1)}(\boldsymbol{\alpha}, \mathbf{u})$ is determined by selecting appropriate adjoint boundary and/or initial conditions, which will be denoted in operator form as:

$$
\left\{\mathbf{B}_{A}^{(1)}\left(\boldsymbol{\alpha}, \mathbf{u} ; \boldsymbol{\psi}^{(1)}\right)\right\}_{\left(\mathbf{e}^{0}\right)}=0, \mathbf{x} \in \partial \Omega_{x}\left(\boldsymbol{\alpha}^{0}\right) .
$$

The above boundary conditions for $\mathbf{A}_{11}^{(1)}(\boldsymbol{\alpha}, \mathbf{u})$ are usually inhomogeneous, i.e., $\mathbf{B}_{A}^{(1)}(\boldsymbol{\alpha}, \mathbf{u} ; 0) \neq 0$, and are obtained by requiring that:

(a) They must be independent of unknown values of $\delta \mathbf{u}$ and $\delta \alpha$;

(b) The substitution of the boundary and/or initial conditions represented by Equations (A8) and (A15) into the expression of $\left\{P^{(1)}\left(\boldsymbol{\alpha}, \mathbf{u} ; \boldsymbol{\psi}^{(1)} ; \delta \mathbf{u}\right)\right\}_{\partial \Omega_{x}}$ must cause all terms containing unknown values of $\delta \mathbf{u}$ to vanish.

Constructing the adjoint initial and/or boundary conditions for $\mathbf{A}_{11}^{(1)}(\boldsymbol{\alpha}, \mathbf{u})$ as described above and implementing them together with the forward boundary and/or initial conditions [represented by Equations (A8)] into Equation (A14) reduces the bilinear concomitant $\left\{P^{(1)}\left(\boldsymbol{\alpha}, \mathbf{u} ; \boldsymbol{\psi}^{(1)} ; \delta \mathbf{u}\right)\right\}_{\partial \Omega_{x}}$ to a quantity that will contain boundary terms involving only known values of $\delta \alpha, \alpha^{0}, \mathbf{u}^{0}$, and $\psi^{(1)}$; this quantity will be denoted by $\hat{P}^{(1)}\left(\alpha, \mathbf{u} ; \psi^{(1)} ; \delta \boldsymbol{\alpha}\right)$. In general, $\hat{P}^{(1)}\left(\alpha, \mathbf{u} ; \boldsymbol{\psi}^{(1)} ; \delta \boldsymbol{\alpha}\right)$ does not automatically vanish as a result of the operations discussed in the foregoing. In certain cases, though, $\hat{P}^{(1)}\left(\boldsymbol{\alpha}, \mathbf{u} ; \boldsymbol{\psi}^{(1)} ; \delta \boldsymbol{\alpha}\right)$ may vanish automatically or it may be forced to vanish by considering appropriately constructed extensions of $\mathbf{A}_{11}^{(1, f \circ r)}(\boldsymbol{\alpha}, \mathbf{u})$; however, such extensions are seldom needed in practice.

Implementing the forward and adjoint boundary and/or initial conditions, cf., Equations (A8) and (A15), into Equation (A14) will transform the later into the following relation:

$$
\left\{\left\langle\mathbf{A}_{11}^{(1)}(\boldsymbol{\alpha}, \mathbf{u}) \boldsymbol{\psi}^{(1)}, \delta \mathbf{u}\right\rangle_{u}\right\}_{\left(\mathbf{e}^{0}\right)}=\left\{\left\langle\boldsymbol{\psi}^{(1)}, \mathbf{F}_{11}^{(1)}(\boldsymbol{\alpha}, \mathbf{u}) \delta \mathbf{u}\right\rangle_{Q}\right\}_{\left(\mathbf{e}^{0}\right)}-\left\{\hat{P}^{(1)}\left(\boldsymbol{\alpha}, \mathbf{u} ; \boldsymbol{\psi}^{(1)} ; \delta \boldsymbol{\alpha}\right)\right\}_{\left(\mathbf{e}^{0}\right)} .
$$

The quantity $\mathbf{F}_{11}^{(1)}\left(\boldsymbol{\alpha}^{0}, \mathbf{u}^{0}\right) \delta \mathbf{u}$ in the first term on the right-side of Equation (A16) is now replaced by using the right-side of Equation (A7) to obtain:

$$
\left\{\left\langle\mathbf{A}_{11}^{(1)}(\boldsymbol{\alpha}, \mathbf{u}) \boldsymbol{\psi}^{(1)}, \delta \mathbf{u}\right\rangle_{u}\right\}_{\left(\mathbf{e}^{0}\right)}=\left\{\left\langle\boldsymbol{\psi}^{(1)}, \mathbf{Q}^{(1)}(\boldsymbol{\alpha}, \mathbf{u} ; \delta \boldsymbol{\alpha})\right\rangle_{Q}\right\}_{\left(\mathbf{e}^{0}\right)}-\left\{\hat{P}^{(1)}\left(\boldsymbol{\alpha}, \mathbf{u} ; \boldsymbol{\psi}^{(1)} ; \delta \boldsymbol{\alpha}\right)\right\}_{\left(\mathbf{e}^{0}\right)} .
$$

Since $\psi^{(1)}$ is not completely defined yet; its definition will now be completed by requiring that the left-side of Equation (A17) and the right side of Equation (A6) represent the same functional, i.e., the "indirect effect term" $\left\{\delta R\left(\mathbf{e}^{0} ; \mathbf{h}\right)\right\}_{\text {ind }}$. This requirement is achieved by imposing the following relationship:

$$
\left\{\left\langle\mathbf{A}_{11}^{(1)}(\boldsymbol{\alpha}, \mathbf{u}) \boldsymbol{\psi}^{(1)}, \delta \mathbf{u}\right\rangle_{u}\right\}_{\left(\mathbf{e}^{0}\right)}=\left\{\langle\partial S(\boldsymbol{\alpha}, \mathbf{u}) / \partial \mathbf{u}, \delta \mathbf{u}\rangle_{u}\right\}_{\left(\mathbf{e}^{0}\right)}, \delta \mathbf{u} \in H_{u} .
$$


which implies that the 1 st-level adjoint function $\psi^{(1)}$ is the weak solution (in the sense of distributions) of the following equation:

$$
\left\{\mathbf{A}_{11}^{(1)}(\boldsymbol{\alpha}, \mathbf{u}) \boldsymbol{\psi}^{(1)}(\mathbf{x})\right\}_{\left(\mathbf{e}^{0}\right)}=\left\{\frac{\partial S(\boldsymbol{\alpha}, \mathbf{u})}{\partial \mathbf{u}}\right\}_{\left(\mathbf{e}^{0}\right)}, \mathbf{x} \in \Omega_{x}
$$

while satisfying the adjoint boundary conditions represented by Equation (A15). As discussed in References [2,3,50], the well-known Riesz representation theorem ensures that the relationship in Equation (A19) holds uniquely. The results obtained in Equations (17), (21) and (22) are now replaced in Equation (15) to obtain:

$$
\{\delta R(\mathbf{e} ; \mathbf{h})\}_{\left(\mathbf{e}^{0}\right)}=\left\{\frac{\partial R(\boldsymbol{\alpha}, \mathbf{u})}{\partial \boldsymbol{\alpha}}\right\}_{\left(\mathbf{e}^{0}\right)} \delta \boldsymbol{\alpha}+\left\{\left\langle\boldsymbol{\psi}^{(1)}, \mathbf{Q}^{(1)}(\boldsymbol{\alpha}, \mathbf{u} ; \delta \boldsymbol{\alpha})\right\rangle_{Q}\right\}_{\left(\mathbf{e}^{0}\right)}-\left\{\hat{P}^{(1)}\left(\boldsymbol{\alpha}, \mathbf{u} ; \boldsymbol{\psi}^{(1)} ; \delta \boldsymbol{\alpha}\right)\right\}_{\left(\mathbf{e}^{0}\right)} .
$$

As the right-side of Equationindicates, the desired elimination of all unknown values of $\delta \mathbf{u}$ from the expression of total first-differential of $R(\mathbf{e})$ at $\mathbf{e}^{0}$ has been accomplished. Instead of depending on $\delta \mathbf{u}$, the 1st-order response differential $\delta R\left(\mathbf{e}^{0} ; \mathbf{h}\right)$ now depends on the adjoint function $\psi^{(1)} \in \mathrm{H}_{Q}$, a fact that is emphasized in the above expression by noting explicitly the identity $\delta R\left(\mathbf{e}^{0} ; \mathbf{h}\right) \equiv D R\left(\boldsymbol{\alpha}^{0}, \mathbf{u}^{0} ; \boldsymbol{\psi}^{(1)} ; \delta \boldsymbol{\alpha}\right)$, where $\left\{D R\left(\boldsymbol{\alpha}, \mathbf{u} ; \boldsymbol{\psi}^{(1)} ; \delta \boldsymbol{\alpha}\right)\right\}_{\left(\mathbf{e}^{0}\right)}$ denotes the total first-order differential of the response $R(\mathbf{e})$ with respect to the parameters $\alpha$. Furthermore, to emphasize that $\mathrm{H}_{Q}\left(\Omega_{x}\right)$ becomes the Hilbert space on which the adjoint function $\boldsymbol{\psi}^{(1)} \in \mathrm{H}_{Q}$ is defined, the Hilbert space $\mathrm{H}_{Q}$ will henceforth be denoted as $\mathrm{H}_{\psi}^{(1)}\left(\Omega_{x}\right)$ and the inner product $\langle\bullet, \bullet\rangle_{Q}$ will be denoted as $\langle\bullet, \bullet\rangle_{\psi^{(1)}}$, with $\boldsymbol{\psi}^{(1)} \in \mathrm{H}_{\psi}^{(1)}\left(\Omega_{x}\right)$. This notation will be convenient for the subsequent developments, to be presented in the next Section, below, of the higher-level adjoint sensitivity systems, in the sense that the 2nd-level adjoint sensitivity systems will involve an adjoint function that will be designated as $\psi^{(2)} \in \mathrm{H}_{\psi}^{(2)}\left(\Omega_{x}\right)$, and an inner product in $\mathrm{H}_{\psi}^{(2)}\left(\Omega_{x}\right)$ which will be denoted as $\langle\bullet, \bullet\rangle_{\psi^{(2)}}$.

As indicated by Equation (A20), the total 1st-order response variation $D R\left(\boldsymbol{\alpha}^{0}, \mathbf{u}^{0} ; \boldsymbol{\psi}^{(1)} ; \delta \boldsymbol{\alpha}\right)$ can be computed after solving Equations (A15) and (A19) only once to obtain the adjoint function $\psi^{(1)} \in \mathrm{H}_{\psi}^{(1)}\left(\Omega_{x}\right)$. Equations (A15) and (A19) will be called the 1st-Level Adjoint Sensitivity System (1st-LASS), and its solution, $\boldsymbol{\psi}^{(1)} \in \mathrm{H}_{\psi}^{(1)}\left(\Omega_{x}\right)$, will be called the 1st-level adjoint function. It is very important to note that the 1st-LASS is independent of the variation $\delta \mathbf{u}$ in the original state functions $\mathbf{u}$.

The individual sensitivities of the response $R(\mathbf{e})$ with respect to each of the model parameters $\alpha_{i}$, evaluated at the nominal values $\mathbf{e}^{0}=\left(\boldsymbol{\alpha}^{0}, \mathbf{u}^{0}\right)$ are obtained by successively setting $\left(\delta \alpha_{i}=1, \delta \alpha_{j}=0\right.$ for $\left.j \neq i, i=1, \ldots, N_{\alpha}\right)$ in the expression of $\delta R\left(\mathbf{e}^{0} ; \mathbf{h}\right) \equiv$ $D R\left(\boldsymbol{\alpha}^{0}, \mathbf{u}^{0} ; \boldsymbol{\psi}^{(1)} ; \delta \boldsymbol{\alpha}\right)$ given in Equation (A20) and performing the simple integrations to determine the inner-product term.

In the vast majority of practical situations, the G-differential $\delta \mathbf{Q}\left(\boldsymbol{\alpha}^{0} ; \delta \boldsymbol{\alpha}\right)$ of the source term $\mathbf{Q}\left(\boldsymbol{\alpha}^{0}\right)$ is linear in $\delta \boldsymbol{\alpha}$, which implies that

$$
\mathbf{Q}^{(1)}\left(\boldsymbol{\alpha}^{0}, \mathbf{u}^{0} ; \delta \boldsymbol{\alpha}\right)=\sum_{i_{1}=1}^{N_{\alpha}} \frac{\partial\left[\mathbf{Q}\left(\boldsymbol{\alpha}^{0}\right)-\mathbf{N}\left(\boldsymbol{\alpha}^{0}, \mathbf{u}^{0}\right)\right]}{\partial \alpha_{i_{1}}} \delta \alpha_{i_{1}} .
$$

Since $\hat{P}\left(\boldsymbol{\alpha}^{0}, \mathbf{u}^{0} ; \boldsymbol{\psi}^{(1)} ; \delta \boldsymbol{\alpha}\right)$ is also linear in $\delta \boldsymbol{\alpha}$, it follows that it can be expressed as

$$
\hat{P}^{(1)}\left(\boldsymbol{\alpha}^{0}, \mathbf{u}^{0} ; \boldsymbol{\psi}^{(1)} ; \delta \boldsymbol{\alpha}\right)=\sum_{i_{1}=1}^{N_{\alpha}} \frac{\partial \hat{P}^{(1)}\left(\boldsymbol{\alpha}^{0}, \mathbf{u}^{0} ; \boldsymbol{\psi}^{(1)}\right)}{\partial \alpha_{i_{1}}} \delta \alpha_{i_{1}} .
$$


Therefore, Equation (A12) can be written in the form already anticipated in Equation (A19), namely

$$
\{\delta R(\mathbf{e} ; \mathbf{h})\}_{\left(\mathbf{e}^{0}\right)} \equiv\left\{D R\left(\boldsymbol{\alpha}, \mathbf{u} ; \boldsymbol{\psi}^{(1)} ; \delta \boldsymbol{\alpha}\right)\right\}_{\left(\mathbf{e}^{0}\right)}=\sum_{i_{1}=1}^{N_{\alpha}}\left\{R_{i_{1}}^{(1)}\left(\boldsymbol{\alpha}, \mathbf{u} ; \boldsymbol{\psi}^{(1)}\right)\right\}_{\left(\mathbf{e}^{0}\right)} \delta \alpha_{i_{1}},
$$

where the quantities $R_{i_{1}}^{(1)}\left(\boldsymbol{\alpha} ; \mathbf{u} ; \boldsymbol{\psi}^{(1)}\right)$ denote the 1st-order partial sensitivities (G-derivatives) of the response $R(\mathbf{e})$ with respect to each of the model parameters $\alpha_{i}$, evaluated at $\alpha^{0}, \mathbf{u}^{0}, \boldsymbol{\psi}^{(1)}$, and are given by the following expression:

$$
\begin{aligned}
& R_{i_{1}}^{(1)}\left(\boldsymbol{\alpha}, \mathbf{u} ; \boldsymbol{\psi}^{(1)}\right) \triangleq \frac{\partial R(\boldsymbol{\alpha}, \mathbf{u})}{\partial \alpha_{i_{1}}}-\frac{\partial \hat{P}^{(1)}\left(\boldsymbol{\alpha}, \mathbf{u} ; \boldsymbol{\Psi}^{(1)}\right)}{\partial \alpha_{i_{1}}} \\
& +\int_{\Omega_{x}} \boldsymbol{\psi}^{(1)}(\mathbf{x}) \cdot\left\{\frac{\partial[\mathbf{Q}(\boldsymbol{\alpha})-\mathbf{N}(\boldsymbol{\alpha}, \mathbf{u})]}{\partial \alpha_{i_{1}}}\right\} d \mathbf{x} ; \quad i_{1}=1, \ldots, T P .
\end{aligned}
$$

For subsequent generalizations to higher levels and orders, the standardized notation for the 1st-LASS is summarized below, keeping in mind that the respective operator equations hold in the distributional (weak) sense:

$$
\begin{gathered}
\mathbf{A}_{11}^{(1)}(\boldsymbol{\alpha}, \mathbf{u}) \boldsymbol{\psi}^{(1)}(\mathbf{x})=\Theta^{(1)}(\boldsymbol{\alpha}, \mathbf{u}), \Theta^{(1)}(\boldsymbol{\alpha}, \mathbf{u}) \triangleq \frac{\partial S(\boldsymbol{\alpha}, \mathbf{u})}{\partial \mathbf{u}}, \mathbf{x} \in \Omega_{x}, \boldsymbol{\psi}^{(1)} \in H_{\psi}^{(1)}\left(\Omega_{x}\right), \\
\mathbf{B}_{A}^{(1)}\left(\boldsymbol{\alpha}, \mathbf{u} ; \boldsymbol{\psi}^{(1)}\right)=0, \mathbf{x} \in \partial \Omega_{x}(\boldsymbol{\alpha}),
\end{gathered}
$$

where the superscript "(1)" indicates "1st-Level," the letter " $\mathrm{A}$ " (used as "operator" and "subscript") indicates "Adjoint," and the letter "B" indicates "boundary and/or initial conditions."

Since real Hilbert spaces provide the natural mathematical setting for computational purposes, the derivations presented in this Section are set in real (as opposed to complex) Hilbert spaces, without affecting the generality of the concepts presented here.

Appendix A.2. The Second-Order Comprehensive Adjoint Sensitivity Analysis Methodology for Nonlinear Systems (2nd-CASAM-N)

The 2 nd-order total sensitivity of the model response $R(\mathbf{e})$ to variations in the model parameters and state functions around $\mathbf{e}^{0}=\left(\alpha^{0}, \mathbf{u}^{0}\right)$ will be assumed to exist, will be denoted as $\delta^{(2)} R\left(\mathbf{e}^{0} ; \mathbf{h}_{1}, \mathbf{h}_{2}\right)$, and will be defined inductively as "the total first-variation of the 1st-order total response variation," namely:

$$
\delta^{(2)} R\left(\mathbf{e}^{0} ; \mathbf{h}_{1}, \mathbf{h}_{2}\right) \triangleq\left\{\frac{d}{d \varepsilon_{1}}\left[\delta R\left(\mathbf{e}^{0}+\varepsilon_{1} \mathbf{h}_{1} ; \mathbf{h}_{2}\right)\right]\right\}_{\varepsilon_{1}=0}=\left\{\frac{\partial^{2}}{\partial \varepsilon_{1} \partial \varepsilon_{2}}\left[R\left(\mathbf{e}^{0}+\varepsilon_{1} \mathbf{h}_{1}+\varepsilon_{2} \mathbf{h}_{2}\right)\right]\right\}_{\varepsilon_{1}=\varepsilon_{2}=0},
$$

for $\varepsilon_{1}, \varepsilon_{2} \in \mathrm{F}$, and all (i.e., arbitrary) vectors of variations $\mathbf{h}_{i} \in \mathrm{E}$; $i=1,2$; around $\mathbf{e}^{0}=\left(\boldsymbol{\alpha}^{0}, \mathbf{u}^{0}\right) \in$ E. In order for the 2nd-order response sensitivities (i.e., 2nd-order partial G-derivatives) to model parameters to exist, the 1st-order sensitivities must satisfy conditions equivalent to those expressed in Equations (A2) and (A3). Assuming henceforth that these conditions are satisfied, the total 2nd-order response differential $\delta^{(2)} R\left(\mathbf{e}^{0} ; \mathbf{h}_{1}, \mathbf{h}_{2}\right)$ is bilinear in the parameter variations $\delta \alpha_{i}$, a fact that will be denoted as $D^{(2)} R\left(\mathbf{e}^{0} ; \mathbf{h}_{1}, \mathbf{h}_{2}\right)$, so that Equation (A27) will ultimately be expressed in the following form:

$$
D^{(2)} R\left(\mathbf{e}^{0} ; \mathbf{h}_{1}, \mathbf{h}_{2}\right)=\sum_{i_{2}, i_{1}=1}^{N_{\alpha}} R_{i_{2} i_{1}}^{(2)} \delta \alpha_{i_{2}} \delta \alpha_{i_{1}}
$$

In Equation (A28), the quantities $R_{i_{2} i_{1}}^{(2)}$ do not depend on the parameter variations and represent the mixed 2 nd-order partial sensitivities ( $G$-derivatives) of the model response $R(\mathbf{e})$ at $\mathbf{e}^{0}$. The exact and efficient computation of the 2 nd-order partial sensitivities (G- 
derivatives) $R_{i_{2} i_{1}}^{(2)}$ will be the objective of the developments to be presented in the remainder of this sub-section.

As Equation (A28) indicates, the 1st-order partial sensitivities are functionals of the form $R_{i_{1}}^{(1)}\left(\boldsymbol{\alpha}, \mathbf{u} ; \boldsymbol{\psi}^{(1)}\right) \in \mathrm{H}_{\alpha} \oplus \mathrm{H}_{u}\left(\Omega_{x}\right) \oplus \mathrm{H}_{\psi}^{(1)}\left(\Omega_{x}\right) \rightarrow \mathrm{F}$. The product Hilbert-space $\mathrm{H}_{\psi}^{(1)}\left(\Omega_{x}\right) \oplus \mathrm{H}_{u}\left(\Omega_{x}\right)$ will play a prominent role in the derivations to follow. For notational convenience, therefore, it will be denoted as $\mathrm{H}_{\psi}^{(2)}\left(\Omega_{x}\right) \triangleq \mathrm{H}_{\psi}^{(1)}\left(\Omega_{x}\right) \oplus \mathrm{H}_{u}\left(\Omega_{x}\right)$. An arbitrary point in $\mathrm{H}_{\psi}^{(2)}\left(\Omega_{x}\right)$ will be denoted as $\boldsymbol{\varphi}^{(2)} \triangleq\left(\mathbf{u} ; \boldsymbol{\psi}^{(1)}\right) \in \mathrm{H}_{u}\left(\Omega_{x}\right) \oplus \mathrm{H}_{\psi}^{(1)}\left(\Omega_{x}\right)$, with the respective "nominal value" denoted as $\boldsymbol{\varphi}^{(2,0)} \triangleq\left(\mathbf{u}^{0} ; \boldsymbol{\psi}^{(1,0)}\right) \in \mathrm{H}_{u}\left(\Omega_{x}\right) \oplus \mathrm{H}_{\psi}^{(1)}\left(\Omega_{x}\right)$. A vector of arbitrary variations around $\boldsymbol{\varphi}^{(2,0)} \triangleq\left(\mathbf{u}^{0} ; \boldsymbol{\psi}^{(1,0)}\right)$ will be denoted as $\delta \boldsymbol{\varphi}^{(2)} \triangleq\left(\delta \mathbf{u} ; \delta \boldsymbol{\psi}^{(1)}\right)$. Occasionally, however, the respective arguments of $\varphi^{(2)}, \varphi^{(2,0)}$ and $\delta \varphi^{(2)}$ may be written out explicitly, whenever needed for clarity.

For each $i_{1}=1, \ldots, T P$, the 1 st-order G-differential $\delta R_{i_{1}}^{(1)}\left(\boldsymbol{\alpha}^{0}, \varphi^{(2,0)} ; \delta \boldsymbol{\alpha}, \delta \varphi^{(2)}\right)$ of $R_{i_{1}}^{(1)}\left(\alpha ; \varphi^{(2)}\right)$ can be computed in the neighborhood of the point $\left(\alpha^{0}, \varphi^{(2,0)}\right)$, in the usual manner, namely:

$$
\begin{aligned}
\delta R_{i_{1}}^{(1)}\left(\boldsymbol{\alpha}^{0}, \boldsymbol{\varphi}^{(2,0)} ; \delta \boldsymbol{\alpha}, \delta \boldsymbol{\varphi}^{(2)}\right) & \triangleq\left\{\frac{d}{d \varepsilon} R_{i_{1}}^{(1)}\left(\boldsymbol{\alpha}^{0}+\varepsilon \delta \boldsymbol{\alpha}, \mathbf{u}^{0}+\varepsilon \delta \mathbf{u} ; \boldsymbol{\psi}^{(1,0)}+\varepsilon \delta \boldsymbol{\psi}^{(1)}\right)\right\}_{\varepsilon=0} \\
= & \left(\delta R_{i_{1}}^{(1)}\right)_{\text {direct }}+\left(\delta R_{i_{1}}^{(1)}\right)_{\text {indirect }}
\end{aligned}
$$

where the "direct-effect term" $\left(\delta R_{i_{1}}^{(1)}\right)_{\text {direct }}$ is defined as follows:

$$
\begin{aligned}
& \left(\delta R_{i_{1}}^{(1)}\right)_{\text {direct }} \triangleq \sum_{i_{2}=1}^{N_{\boldsymbol{\alpha}}}\left\{\frac{\partial R_{i_{1}}^{(1)}\left(\boldsymbol{\alpha}, \boldsymbol{\varphi}^{(2)}\right)}{\partial \alpha_{i_{2}}}\right\}_{\left(\boldsymbol{\alpha}^{0}, \boldsymbol{\varphi}^{(2,0)}\right)} \delta \alpha_{i_{2}}, i_{1}=1, \ldots, T P, \\
& \frac{\partial R_{i_{1}}^{(1)}\left(\boldsymbol{\alpha}, \boldsymbol{\varphi}^{(2)}\right)}{\partial \alpha_{i_{2}}}=\frac{\partial R(\boldsymbol{\alpha}, \mathbf{u})}{\partial \alpha_{i_{1}}}+\left\langle\boldsymbol{\psi}^{(1)}, \frac{\partial \mathbf{Q}(\boldsymbol{\alpha})-\mathbf{N}(\boldsymbol{\alpha}, \mathbf{u})]}{\partial \alpha_{i_{1}}}\right\rangle_{\psi^{(1)}}-\frac{\partial \hat{P}^{(1)}\left(\boldsymbol{\alpha}, \mathbf{u} ; \boldsymbol{\psi}^{(1)}\right)}{\partial \alpha_{i_{1}}} .
\end{aligned}
$$

and where the "indirect-effect term" $\left(\delta R_{i_{1}}^{(1)}\right)_{\text {indirect }}$ is defined as follows:

$$
\left(\delta R_{i_{1}}^{(1)}\right)_{\text {indirect }} \triangleq\left\{\frac{\partial R_{i_{1}}^{(1)}}{\partial \boldsymbol{\psi}^{(1)}} \delta \boldsymbol{\psi}^{(1)}+\frac{\partial R_{i_{1}}^{(1)}}{\partial \mathbf{u}} \delta \mathbf{u}\right\}_{\left(\boldsymbol{\alpha}^{0}, \boldsymbol{\varphi}^{(2,0)}\right)}=\left\{\left(\delta \boldsymbol{\psi}^{(1)}, \delta \mathbf{u}\right)\left(\begin{array}{c}
\boldsymbol{\Theta}_{1, i_{1}}^{(2)}\left(\boldsymbol{\alpha}, \boldsymbol{\varphi}^{(2)}\right. \\
\boldsymbol{\Theta}_{2, i_{1}}^{(2)}\left(\boldsymbol{\alpha}, \boldsymbol{\varphi}^{(2)}\right.
\end{array}\right)\right\}_{\left(\boldsymbol{\alpha}^{0}, \boldsymbol{\varphi}^{(2,0)}\right)},
$$

where

$$
\begin{gathered}
\boldsymbol{\Theta}_{1, i_{1}}^{(2)}\left(\boldsymbol{\alpha}, \boldsymbol{\varphi}^{(2)}\right) \triangleq \frac{\partial R_{i_{1}}^{(1)}\left(\boldsymbol{\alpha}, \mathbf{u} ; \boldsymbol{\psi}^{(1)}\right)}{\partial \boldsymbol{\psi}^{(1)}}=\frac{\partial}{\partial \alpha_{i_{1}}}\left[\mathbf{Q}(\boldsymbol{\alpha})-\mathbf{N}(\boldsymbol{\alpha}, \mathbf{u})-\frac{\partial \hat{P}^{(1)}\left(\boldsymbol{\alpha}, \mathbf{u} ; \boldsymbol{\psi}^{(1)}\right)}{\partial \boldsymbol{\psi}^{(1)}}\right] \\
\boldsymbol{\Theta}_{2, i_{1}}^{(2)}\left(\boldsymbol{\alpha}, \boldsymbol{\varphi}^{(2)}\right) \triangleq \frac{\partial R_{i_{1}}^{(1)}\left(\boldsymbol{\alpha}, \mathbf{u} ; \boldsymbol{\psi}^{(1)}\right)}{\partial \mathbf{u}}=\frac{\partial}{\partial \alpha_{i_{1}}}\left[\Theta^{(1)}(\boldsymbol{\alpha}, \mathbf{u})-\boldsymbol{\Lambda}\left(\boldsymbol{\alpha}, \mathbf{u} ; \boldsymbol{\psi}^{(1)}\right)-\frac{\partial \hat{P}^{(1)}\left(\boldsymbol{\alpha}, \mathbf{u} ; \boldsymbol{\psi}^{(1)}\right)}{\partial \mathbf{u}}\right], \\
\boldsymbol{\Lambda}\left(\boldsymbol{\alpha}, \mathbf{u} ; \boldsymbol{\psi}^{(1)}\right) \triangleq\left(\Lambda_{1}, \ldots, \Lambda_{N_{u}}\right) ; \Lambda_{k}\left(\boldsymbol{\alpha}, \mathbf{u} ; \boldsymbol{\psi}^{(1)}\right) \triangleq \sum_{j=1}^{N_{u}} \psi_{j}^{(1)}(\mathbf{x}) \frac{\partial N_{j k}(\boldsymbol{\alpha}, \mathbf{u})}{\partial \mathbf{u}} ; k=1, \ldots, N_{u}
\end{gathered}
$$

Since the "direct-effect term" $\left(\delta R_{i_{1}}^{(1)}\right)_{\text {direct }}$ is independent of variations $\delta \varphi^{(2)} \triangleq$ $\left(\delta \mathbf{u} ; \delta \boldsymbol{\psi}^{(1)}\right)$, it can be computed immediately at this stage, without needing any additional large-scale computations. On the other hand, the "indirect-effect term" $\left(\delta R_{i_{1}}^{(1)}\right)_{\text {indirect }}$ cannot be computed at this stage yet, since the vectors of variations $\delta \mathbf{u}$ and $\delta \boldsymbol{\psi}^{(1)}$ are not completely known. The vector of variations $\delta \psi^{(1)}$ is the solution of the system of operator equations 
that will result after G-differentiating the 1st-LASS given by Equations (A25) and (A26) in the neighborhood $\left(\boldsymbol{\alpha}^{0}+\varepsilon \delta \boldsymbol{\alpha}, \mathbf{u}^{0}+\varepsilon \delta \mathbf{u} ; \boldsymbol{\psi}^{(1,0)}+\varepsilon \delta \boldsymbol{\psi}^{(1)}\right)$ of the point $\left(\boldsymbol{\alpha}^{0}, \boldsymbol{\varphi}^{(2,0)}\right)$. This G-differentiation yields the following system of operator equations:

$$
\begin{gathered}
\left\{\mathrm{F}_{11}^{(2)}(\boldsymbol{\alpha} ; \mathbf{u}) \delta \boldsymbol{\psi}^{(1)}+\mathrm{F}_{12}^{(2)}(\boldsymbol{\alpha} ; \mathbf{u}) \delta \mathbf{u}\right\}_{\left(\boldsymbol{\alpha}^{0}, \boldsymbol{\varphi}^{(2,0)}\right)}=\left\{\mathbf{Q}^{(2)}\left(\boldsymbol{\alpha}, \boldsymbol{\varphi}^{(2)} ; \delta \boldsymbol{\alpha}\right)\right\}_{\left(\boldsymbol{\alpha}^{0}, \boldsymbol{\varphi}^{(2,0)}\right)^{\prime}} \\
\left\{\mathbf{B}_{A}^{(1)}\left(\boldsymbol{\alpha}, \boldsymbol{\varphi}^{(2)} ; \delta \boldsymbol{\alpha}, \delta \boldsymbol{\psi}^{(1)}\right)\right\}_{\left(\boldsymbol{\alpha}^{0}, \boldsymbol{\varphi}^{(2,0)}\right)}=0, \mathbf{x} \in \partial \Omega_{x}\left(\boldsymbol{\alpha}^{0}\right) .
\end{gathered}
$$

where the following definitions were used:

$$
\mathrm{F}_{11}^{(2)}(\boldsymbol{\alpha}, \mathbf{u}) \triangleq \mathbf{A}_{11}^{(1)}(\boldsymbol{\alpha}, \mathbf{u}) ; \mathrm{F}_{12}^{(2)}(\boldsymbol{\alpha} ; \mathbf{u}) \triangleq-\frac{\partial \Theta^{(1)}(\boldsymbol{\alpha}, \mathbf{u})}{\partial \mathbf{u}} ; \mathbf{Q}^{(2)}\left(\boldsymbol{\alpha}, \boldsymbol{\varphi}^{(2)} ; \delta \boldsymbol{\alpha}\right) \triangleq \frac{\partial\left[\Theta^{(1)}(\boldsymbol{\alpha}, \mathbf{u})-\mathbf{A}_{11}^{(1)}(\boldsymbol{\alpha}, \mathbf{u}) \boldsymbol{\psi}^{(1)}\right]}{\partial \boldsymbol{\alpha}} \delta \boldsymbol{\alpha}
$$

As Equations (A35) and (A36) indicate, the vector of variations $\delta \psi^{(1)}$ depends on the parameters variations $\delta \alpha$. To construct a well-posed system of equations for obtaining both $\delta \boldsymbol{\psi}^{(1)}$ and $\delta \mathbf{u}$ in terms of $\delta \boldsymbol{\alpha}$, the 1st-LFSS given by Equations (A7) and (A8) is combined together with Equations (A35) and (A36) to obtain the following block matrix system:

$$
\left\{\left(\begin{array}{cc}
\mathbf{F}_{11}^{(2)}(\boldsymbol{\alpha}, \mathbf{u}) & \mathbf{F}_{12}^{(2)}(\boldsymbol{\alpha}, \mathbf{u}) \\
0 & \mathbf{F}_{11}^{(1)}(\boldsymbol{\alpha}, \mathbf{u})
\end{array}\right)\left(\begin{array}{c}
\delta \boldsymbol{\psi}^{(1)} \\
\delta \mathbf{u}
\end{array}\right)\right\}_{\left(\boldsymbol{\alpha}^{0}, \boldsymbol{\varphi}^{(2,0)}\right)}=\left\{\left(\begin{array}{c}
\mathbf{Q}^{(2)}\left(\boldsymbol{\alpha}, \boldsymbol{\varphi}^{(2)} ; \delta \boldsymbol{\alpha}\right) \\
\mathbf{Q}^{(1)}(\boldsymbol{\alpha}, \mathbf{u} ; \delta \boldsymbol{\alpha})
\end{array}\right)\right\}_{\left(\boldsymbol{\alpha}^{0}, \boldsymbol{\varphi}^{(2,0)}\right)}, \mathbf{x} \in \Omega_{x},
$$

together with the following boundary and/or initial conditions:

$$
\left\{\mathbf{B}_{F}^{(2)}\left(\boldsymbol{\alpha}, \boldsymbol{\varphi}^{(2)} ; \delta \boldsymbol{\alpha}, \delta \boldsymbol{\varphi}^{(2)}\right)\right\}_{\left(\boldsymbol{\alpha}^{0}, \boldsymbol{\varphi}^{(2,0)}\right)} \triangleq\left\{\left(\begin{array}{c}
\delta \mathbf{B}_{A}^{(1)}\left(\boldsymbol{\alpha}, \boldsymbol{\varphi}^{(2)} ; \delta \boldsymbol{\alpha}, \delta \boldsymbol{\varphi}^{(2)}\right) \\
\mathbf{B}_{F}^{(1)}(\boldsymbol{\alpha}, \mathbf{u} ; \delta \boldsymbol{\alpha}, \delta \mathbf{u})
\end{array}\right)\right\}_{\left(\boldsymbol{\alpha}^{0}, \boldsymbol{\varphi}^{(2,0)}\right)}=0, \mathbf{x} \in \partial \Omega_{x}\left(\boldsymbol{\alpha}^{0}\right) .
$$

Equations (A38) and (A39) constitute the 2nd-Level Variational Sensitivity System (2ndLVSS). They can be solved, in principle, to obtain the vectors $\delta \mathbf{u}$ and $\delta \boldsymbol{\psi}^{(1)}$ as functions of the vector of parameter variations $\delta \alpha$, and could subsequently be replaced in in Equation (A31) to determine the indirect effect term $\left(\delta R_{i_{1}}^{(1)}\right)_{\text {indirect }}$. This procedure would be impractical to use for large-scale systems since it would require $O\left(T P^{2}\right)$ large-scale computations.

The 2nd-Level Adjoint Sensitivity System" (2nd-LASS) will be developed using the same guiding principles as those underlying the development of the 1st-LASS. The goal of the $2 n d-L A S S$ is to compute the indirect effect term $\left(\delta R_{i_{1}}^{(1)}\right)_{\text {indirect }}$ in a manner that circumvents the need for computing explicitly the vectors of variations $\delta \psi^{(1)}$ and $\delta \mathbf{u}$. Recall that an element in the Hilbert space $\mathrm{H}_{\psi}^{(2)}\left(\Omega_{x}\right) \triangleq \mathrm{H}_{\psi}^{(1)}\left(\Omega_{x}\right) \oplus \mathrm{H}_{u}\left(\Omega_{x}\right)$ is a 2-block vector of the form $\boldsymbol{\psi}_{i_{1}}^{(2)}(\mathbf{x}) \triangleq\left[\boldsymbol{\psi}_{1, i_{1}}^{(2)}(\mathbf{x}), \boldsymbol{\psi}_{2, i_{1}}^{(2)}(\mathbf{x})\right]^{\dagger} \in \mathrm{H}_{\psi}^{(2)}\left(\Omega_{x}\right)$, with $\boldsymbol{\psi}_{1, i_{1}}^{(2)} \in \mathrm{H}_{\psi}^{(1)}\left(\Omega_{x}\right), \quad \boldsymbol{\psi}_{2, i_{1}}^{(2)} \in \mathrm{H}_{u}\left(\Omega_{x}\right)$. Since Hilbert spaces are self-dual, the spaces $\mathrm{H}_{\psi}^{(1)}\left(\Omega_{x}\right)$ and $\mathrm{H}_{u}\left(\Omega_{x}\right)$ actually coincide, but the distinct notation will be kept in this section for clarity. The inner product $\langle\bullet, \bullet\rangle_{\psi^{(2)}}$ between any two vectors $\mathbf{a}_{i_{1}}^{(2)} \triangleq\left(\mathbf{a}_{1, i_{1}}^{(2)}, \mathbf{a}_{2, i_{1}}^{(2)}\right) \in \mathrm{H}_{\psi}^{(2)}\left(\Omega_{x}\right)$ and $\mathbf{b}_{i_{1}}^{(2)} \triangleq\left(\mathbf{b}_{1, i_{1}}^{(2)}, \mathbf{b}_{2, i_{1}}^{(2)}\right) \in \mathrm{H}_{\psi}^{(2)}\left(\Omega_{x}\right)$ is defined as follows:

$$
\left\langle\mathbf{a}_{i_{1}}^{(2)}, \mathbf{b}_{i_{1}}^{(2)}\right\rangle_{\psi^{(2)}} \triangleq \int_{\Omega_{x}}\left(\mathbf{a}_{1, i_{1}}^{(2)} \cdot \mathbf{b}_{1, i_{1}}^{(2)}+\mathbf{a}_{2, i_{1}}^{(2)} \cdot \mathbf{b}_{2, i_{1}}^{(2)}\right) d \mathbf{x}=\sum_{j=1}^{2} \int_{\Omega_{x}}\left(\mathbf{a}_{j, i_{1}}^{(2)} \cdot \mathbf{b}_{j, i_{1}}^{(2)}\right) d \mathbf{x}
$$

Using the above definition, construct the inner product of Equation (A38) with a yet undefined vector $\boldsymbol{\psi}_{i_{1}}^{(2)}(\mathbf{x}) \triangleq\left[\boldsymbol{\psi}_{1, i_{1}}^{(2)}(\mathbf{x}), \boldsymbol{\psi}_{2, i_{1}}^{(2)}(\mathbf{x})\right]^{\dagger} \in \mathrm{H}_{\psi}^{(2)}\left(\Omega_{x}\right)$ to obtain: 


$$
\begin{aligned}
& \left\{\left\langle\left(\boldsymbol{\Psi}_{1, i_{1}}^{(2)}, \boldsymbol{\Psi}_{2, i_{1}}^{(2)}\right),\left(\begin{array}{cc}
\mathbf{F}_{11}^{(2)}(\boldsymbol{\alpha}, \mathbf{u}) & \mathbf{F}_{12}^{(2)}(\boldsymbol{\alpha} ; \mathbf{u}) \\
0 & \mathbf{F}_{11}^{(1)}(\boldsymbol{\alpha} ; \mathbf{u})
\end{array}\right)\left(\begin{array}{c}
\delta \boldsymbol{\psi}^{(1)} \\
\delta \mathbf{u}
\end{array}\right)\right\rangle_{\psi^{(2)}}\right\}_{\left(\boldsymbol{\alpha}^{0}, \boldsymbol{\varphi}^{(2,0)}\right)} \\
& =\int_{\Omega_{x}}\left\{\left[\boldsymbol{\Psi}_{1, i_{1}}^{(2)} \cdot \mathbf{Q}^{(2)}\left(\boldsymbol{\alpha}, \boldsymbol{\varphi}^{(2)} ; \delta \boldsymbol{\alpha}\right)+\boldsymbol{\Psi}_{2, i_{1}}^{(2)} \cdot \mathbf{Q}^{(1)}(\boldsymbol{\alpha}, \mathbf{u} ; \delta \boldsymbol{\alpha})\right]\right\}_{\left(\boldsymbol{\alpha}^{0}, \boldsymbol{\varphi}^{(2,0)}\right)} d \mathbf{x}, \mathbf{x} \in \Omega_{x}\left(\boldsymbol{\alpha}^{0}\right) .
\end{aligned}
$$

The inner product on the left-side of Equation (A41) is now further transformed by using the definition of the adjoint operator to obtain

$$
\begin{aligned}
& \left\{\left\langle\left(\boldsymbol{\psi}_{1, i_{1}}^{(2)}, \boldsymbol{\psi}_{2, i_{1}}^{(2)}\right),\left(\begin{array}{cc}
\mathbf{F}_{11}^{(2)}(\boldsymbol{\alpha}, \mathbf{u}) & \mathbf{F}_{12}^{(2)}(\boldsymbol{\alpha} ; \mathbf{u}) \\
0 & \mathbf{F}_{11}^{(1)}(\boldsymbol{\alpha} ; \mathbf{u})
\end{array}\right)\left(\begin{array}{c}
\delta \boldsymbol{\psi}^{(1)} \\
\delta \mathbf{u}
\end{array}\right)\right\rangle_{\psi^{(2)}}\right\}_{\left(\boldsymbol{\alpha}^{0}, \boldsymbol{\varphi}^{(2,0)}\right)} \\
& =\left\{\left\langle\left(\delta \boldsymbol{\psi}^{(1)}, \delta \mathbf{u}\right),\left(\begin{array}{cc}
{\left[\mathbf{F}_{11}^{(2)}(\boldsymbol{\alpha}, \mathbf{u})\right]^{*}} & 0 \\
{\left[\mathbf{F}_{12}^{(2)}(\boldsymbol{\alpha}, \mathbf{u})\right]^{*}} & {\left[\mathbf{F}_{11}^{(1)}(\boldsymbol{\alpha}, \mathbf{u})\right]^{*}}
\end{array}\right)\left(\begin{array}{c}
\boldsymbol{\psi}_{1, i_{1}}^{(2)} \\
\boldsymbol{\psi}_{2, i_{1}}^{(2)}
\end{array}\right)\right\rangle_{\psi^{(2)}}\right\}_{\left(\boldsymbol{\alpha}^{0}, \boldsymbol{\varphi}^{(2,0)}\right)} \\
& +\left\{\left\{P^{(2)}\left[\boldsymbol{\alpha}, \boldsymbol{\varphi}^{(2)} ; \boldsymbol{\psi}_{1, i_{1}}^{(2)}, \boldsymbol{\psi}_{2, i_{1}}^{(2)} ; \delta \mathbf{u} ; \delta \boldsymbol{\psi}^{(1)}\right]\right\}_{\partial \Omega_{x}}\right\}_{\left(\boldsymbol{\alpha}^{0}, \boldsymbol{\varphi}^{(2,0)}\right)} \text {. }
\end{aligned}
$$

In Equation (A42), the symbol [ $]^{*}$ indicates "adjoint", as before, while the quantity $\left\{P^{(2)}\left[\alpha, \varphi^{(2)} ; \psi_{1, i_{1}}^{(2)}, \psi_{2, i_{1}}^{(2)} ; \delta \mathbf{u} ; \delta \boldsymbol{\psi}^{(1)}\right]\right\}_{\partial \Omega_{x}}$ denotes the corresponding bilinear concomitant on $\mathbf{x} \in \partial \Omega_{x}\left(\boldsymbol{\alpha}^{0}\right)$. The domains of the adjoint operators appearing on the rightside of Equation (A42) can now be specified by requiring that the function $\boldsymbol{\psi}_{i_{1}}^{(2)}(\mathbf{x}) \triangleq$ $\left[\boldsymbol{\psi}_{1, i_{1}}^{(2)}(\mathbf{x}), \boldsymbol{\psi}_{2, i_{1}}^{(2)}(\mathbf{x})\right]^{\dagger} \in \mathrm{H}_{\psi}^{(2)}\left(\Omega_{x}\right)$ satisfies adjoint boundary conditions denoted as follows:

$$
\left\{\mathbf{B}_{A}^{(2)}\left(\boldsymbol{\alpha}, \boldsymbol{\varphi}^{(2)} ; \boldsymbol{\psi}_{i_{1}}^{(2)}\right)\right\}_{\left(\boldsymbol{\alpha}^{0}, \boldsymbol{\varphi}^{(2,0)}\right)}=0, \mathbf{x} \in \partial \Omega_{x}\left(\boldsymbol{\alpha}^{0}\right) .
$$

The 2nd-level adjoint boundary/initial conditions represented by Equation (A43) are determined by requiring that: (a) they must be independent of unknown values of $\delta \boldsymbol{\alpha} ; \delta \mathbf{u} ; \delta \boldsymbol{\psi}^{(1)}$; (b) the substitution of the boundary and/or initial conditions represented by Equations (47) and (51) into the expression of $\left\{P^{(2)}\left[\boldsymbol{\alpha}, \boldsymbol{\varphi}^{(2)} ; \boldsymbol{\psi}^{(2)} ; \delta \mathbf{u} ; \delta \boldsymbol{\psi}^{(1)}\right]\right\}_{\partial \Omega_{x}}$ must cause all terms containing unknown values of $\delta \mathbf{u}$ and $\delta \boldsymbol{\psi}^{(1)}$ to vanish.

Implementing the 2nd-level forward and adjoint boundary/initial conditions-namely, Equations (A39) and (A43) - into Equation (A42) will transform the latter into the following form:

$$
\begin{aligned}
& \left\{\left\langle\left(\delta \boldsymbol{\psi}^{(1)}, \delta \mathbf{u}\right),\left(\begin{array}{cc}
{\left[\mathbf{F}_{11}^{(2)}\right]^{*}} & 0 \\
{\left[\mathbf{F}_{12}^{(2)}\right]^{*}} & {\left[\mathbf{F}_{11}^{(1)}\right]^{*}}
\end{array}\right)\left(\begin{array}{c}
\boldsymbol{\psi}_{1, i_{1}}^{(2)} \\
\boldsymbol{\psi}_{2, i_{1}}^{(2)}
\end{array}\right)\right\rangle_{\psi^{(2)}}\right\}_{\left(\boldsymbol{\alpha}^{0}, \boldsymbol{\varphi}^{(2,0)}\right)}=-\left\{\hat{P}^{(2)}\left(\boldsymbol{\alpha}, \boldsymbol{\varphi}^{(2)} ; \boldsymbol{\psi}_{i_{1}}^{(2)} ; \delta \boldsymbol{\alpha}\right)\right\}_{\left(\boldsymbol{\alpha}^{0}, \boldsymbol{\varphi}^{(2,0)}\right)} \\
& +\left\{\left\langle\left(\boldsymbol{\psi}_{1, i_{1}}^{(2)}, \boldsymbol{\psi}_{2, i_{1}}^{(2)}\right),\left(\begin{array}{cc}
\mathbf{F}_{11}^{(2)} & \mathbf{F}_{12}^{(2)} \\
0 & \mathbf{F}_{11}^{(2)}
\end{array}\right)\left(\begin{array}{c}
\delta \boldsymbol{\psi}^{(1)} \\
\delta \mathbf{u}
\end{array}\right)\right\rangle_{\psi^{(2)}}\right\}_{\left(\boldsymbol{\alpha}^{0}, \boldsymbol{\varphi}^{(2,0)}\right)} \cdot
\end{aligned}
$$

The right side of Equation (A41) is now used in the second term on the right side of Equation (A44) to obtain:

$$
\begin{aligned}
& \left\{\left\langle\left(\delta \boldsymbol{\psi}^{(1)}, \delta \mathbf{u}\right),\left(\begin{array}{cc}
{\left[\mathbf{F}_{11}^{(2)}\right]^{*}} & 0 \\
{\left[\mathbf{F}_{12}^{(2)}\right]^{*}} & {\left[\mathbf{F}_{11}^{(1)}\right]^{*}}
\end{array}\right)\left(\begin{array}{c}
\boldsymbol{\psi}_{1, i_{1}}^{(2)} \\
\boldsymbol{\psi}_{2, i_{1}}^{(2)}
\end{array}\right)\right\rangle_{\psi^{(2)}}\right\}_{\left(\boldsymbol{\alpha}^{0}, \boldsymbol{\varphi}^{(2,0)}\right)}=-\left\{\hat{P}^{(2)}\left(\boldsymbol{\alpha}, \boldsymbol{\varphi}^{(2)} ; \boldsymbol{\psi}_{i_{1}}^{(2)} ; \delta \boldsymbol{\alpha}\right)\right\}_{\left(\boldsymbol{\alpha}^{0}, \boldsymbol{\varphi}^{(2,0)}\right)} \\
& \left\{\left\langle\left(\boldsymbol{\psi}_{1, i_{1}}^{(2)} \boldsymbol{\psi}_{2, i_{1}}^{(2)}\right),\left(\begin{array}{c}
\mathbf{Q}^{(2)}\left(\boldsymbol{\alpha}, \boldsymbol{\varphi}^{(2)} ; \delta \boldsymbol{\alpha}\right) \\
\mathbf{Q}^{(1)}(\boldsymbol{\alpha}, \mathbf{u} ; \delta \boldsymbol{\alpha})
\end{array}\right)\right\rangle_{\psi^{(2)}}\right\}_{\left(\boldsymbol{\alpha}^{0}, \boldsymbol{\varphi}^{(2,0)}\right)} .
\end{aligned}
$$

The definition of the 2nd-level adjoint function $\psi_{i_{1}}^{(2)}$ will now be completed by requiring that the left side of Equation (A45) and the right side of Equation (A31) represent 
the same functional, namely the "indirect-effect term," $\left(\delta R_{i_{1}}^{(1)}\right)_{\text {indirect }}$, which yields the following relationship:

$$
\left.\left\{\left\langle\left(\delta \boldsymbol{\psi}^{(1)}, \delta \mathbf{u}\right),\left(\begin{array}{cc}
{\left[\mathbf{F}_{11}^{(2)}\right]^{*}} & 0 \\
{\left[\mathbf{F}_{12}^{(2)}\right]^{*}} & {\left[\mathbf{F}_{11}^{(1)}\right]^{*}}
\end{array}\right)\left(\begin{array}{c}
\boldsymbol{\psi}_{1, i_{1}}^{(2)} \\
\boldsymbol{\psi}_{2, i_{1}}^{(2)}
\end{array}\right)\right\rangle_{\psi^{(2)}}\right\}_{\left(\boldsymbol{\alpha}^{0}, \boldsymbol{\varphi}^{(2,0)}\right)}=\left\{\left\langle\left(\delta \boldsymbol{\psi}^{(1)}, \delta \mathbf{u}\right),\left(\begin{array}{c}
\boldsymbol{\Theta}_{1, i_{1}}^{(2)}\left(\boldsymbol{\alpha}, \boldsymbol{\varphi}^{(2)}\right. \\
\boldsymbol{\Theta}_{2, i_{1}}^{(2)}\left(\boldsymbol{\alpha}, \boldsymbol{\varphi}^{(2)}\right.
\end{array}\right)\right)\right\rangle_{\psi^{(2)}}\right\}_{\left(\boldsymbol{\alpha}^{0}, \boldsymbol{\varphi}^{(2,0)}\right)} .
$$

The result obtained in Equation (A46) implies that the 2nd-level adjoint function $\boldsymbol{\psi}_{i_{1}}^{(2)}(\mathbf{x}) \triangleq\left[\boldsymbol{\psi}_{1, i_{1}}^{(2)}(\mathbf{x}), \boldsymbol{\psi}_{2, i_{1}}^{(2)}(\mathbf{x})\right]^{\dagger} \in \mathrm{H}_{\psi}^{(2)}\left(\Omega_{x}\right)$ is the weak solution of the following operator equation:

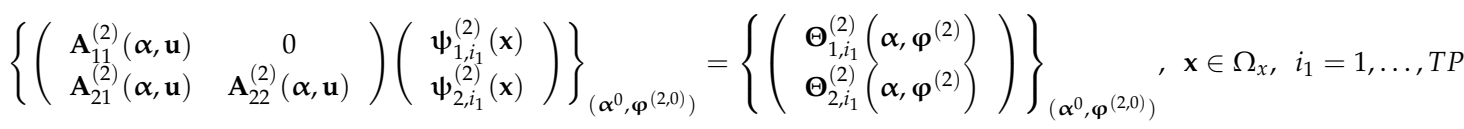

$$
\begin{aligned}
& \mathbf{A}_{11}^{(2)}(\boldsymbol{\alpha}, \mathbf{u}) \triangleq\left[\mathbf{F}_{11}^{(2)}(\boldsymbol{\alpha}, \mathbf{u})\right]^{*}=\left[\mathbf{A}_{11}^{(1)}(\boldsymbol{\alpha}, \mathbf{u})\right]^{*}=\mathbf{F}_{11}^{(1)}(\boldsymbol{\alpha}, \mathbf{u}), \\
& \mathbf{A}_{21}^{(2)}(\boldsymbol{\alpha}, \mathbf{u}) \triangleq\left[\mathbf{F}_{12}^{(2)}(\boldsymbol{\alpha}, \mathbf{u})\right]^{*}, \mathbf{A}_{22}^{(2)}(\boldsymbol{\alpha}, \mathbf{u}) \triangleq\left[\mathbf{F}_{11}^{(1)}(\boldsymbol{\alpha}, \mathbf{u})\right]^{*}=\mathbf{A}_{11}^{(1)}(\boldsymbol{\alpha}, \mathbf{u}) \text {. }
\end{aligned}
$$

Recall that the 2nd-level adjoint function $\boldsymbol{\psi}_{i_{1}}^{(2)}(\mathbf{x}) \triangleq\left[\boldsymbol{\psi}_{1, i_{1}}^{(2)}(\mathbf{x}), \boldsymbol{\psi}_{2, i_{1}}^{(2)}(\mathbf{x})\right]^{\dagger} \in H_{\psi}^{(2)}\left(\Omega_{x}\right)$ satisfies the 2nd-level adjoint boundary conditions represented by Equation (A43).

The results obtained in Equations (A44)-(A47) and (A30) are now replaced in Equation (A29) to obtain:

$$
\begin{aligned}
& \left\{\delta R_{i_{1}}^{(1)}\left(\boldsymbol{\alpha}, \boldsymbol{\varphi}^{(2)} ; \delta \boldsymbol{\alpha}, \delta \mathbf{u}, \delta \boldsymbol{\psi}^{(1)}\right)\right\}_{\left(\boldsymbol{\alpha}^{0}, \boldsymbol{\varphi}^{(2,0)}\right)}=\sum_{i_{2}=1}^{T P}\left\{\frac{\partial R_{i_{1}}^{(1)}\left(\boldsymbol{\alpha}, \boldsymbol{\varphi}^{(2)}\right)}{\partial \alpha_{i_{2}}} \delta \alpha_{i_{2}}\right\}_{\left(\boldsymbol{\alpha}^{0}, \boldsymbol{\varphi}^{(2,0)}\right)} \\
& +\left\{\left\langle\left(\boldsymbol{\psi}_{1, i_{1}}^{(2)}, \boldsymbol{\psi}_{2, i_{1}}^{(2)}\right),\left(\begin{array}{c}
\mathbf{Q}^{(2)}\left(\boldsymbol{\alpha}, \boldsymbol{\varphi}^{(2)} ; \delta \boldsymbol{\alpha}\right) \\
\mathbf{Q}^{(1)}(\boldsymbol{\alpha}, \mathbf{u} ; \delta \boldsymbol{\alpha})
\end{array}\right)\right\rangle_{\psi^{(2)}}\right\}_{\left(\boldsymbol{\alpha}^{0}, \boldsymbol{\varphi}^{(2,0)}\right)}-\left\{\hat{P}^{(2)}\left(\boldsymbol{\alpha}, \boldsymbol{\varphi}^{(2)} ; \boldsymbol{\psi}_{i_{1}}^{(2)} ; \delta \boldsymbol{\alpha}\right)\right\}_{\left(\boldsymbol{\alpha}^{0}, \boldsymbol{\varphi}^{(2,0)}\right)}, i_{1}=1, \ldots, T P .
\end{aligned}
$$

Noting that the quantities $\mathbf{Q}^{(2)}\left(\alpha, \varphi^{(2)} ; \delta \alpha\right)$ and $\hat{P}_{2}\left(\alpha, \varphi^{(2)} ; \psi_{i_{1}}^{(2)} ; \delta \alpha\right)$ are both linear in $\delta \alpha$, it follows that they can be expressed in the following forms:

$$
\begin{gathered}
\left\{\mathbf{Q}^{(2)}\left(\boldsymbol{\alpha}, \boldsymbol{\varphi}^{(2)} ; \delta \boldsymbol{\alpha}\right)\right\}_{\left(\boldsymbol{\alpha}^{0}, \boldsymbol{\varphi}^{(2,0)}\right)}=\sum_{i_{2}=1}^{T P}\left\{\frac{\partial\left[\Theta^{(1)}(\boldsymbol{\alpha}, \mathbf{u})-\mathbf{A}_{11}^{(1)}(\boldsymbol{\alpha}, \mathbf{u}) \boldsymbol{\psi}^{(1)}\right]}{\partial \alpha_{i_{2}}} \delta \alpha_{i_{2}}\right\}_{\left(\boldsymbol{\alpha}^{0}, \boldsymbol{\varphi}^{(2,0)}\right)}, \\
\left\{\hat{P}^{(2)}\left(\boldsymbol{\alpha}, \boldsymbol{\varphi}^{(2)} ; \boldsymbol{\psi}^{(2)} ; \delta \boldsymbol{\alpha}\right)\right\}_{\left(\boldsymbol{\alpha}^{0}, \boldsymbol{\varphi}^{(2,0)}\right)}=\sum_{i_{2}=1}^{T P}\left\{\frac{\partial \hat{P}^{(2)}\left(\boldsymbol{\alpha}, \boldsymbol{\varphi}^{(2)} ; \boldsymbol{\psi}_{1, i_{1},}^{(2)} \boldsymbol{\psi}_{2, i_{1}}^{(2)}\right)}{\partial \alpha_{i_{2}}}\right\}_{\left(\boldsymbol{\alpha}^{0}, \boldsymbol{\varphi}^{(2,0)}\right)} \delta \alpha_{i_{2}} .
\end{gathered}
$$

In view of Equations (A50) and (A51), it follows that Equation (A49) can be expressed as follows:

$$
\delta R_{i_{1}}^{(1)}\left(\boldsymbol{\alpha}, \boldsymbol{\varphi}^{(2)} ; \delta \boldsymbol{\alpha}, \delta \mathbf{u}, \delta \boldsymbol{\psi}^{(1)}\right)=\delta R_{i_{1}}^{(1)}\left(\boldsymbol{\alpha}, \boldsymbol{\varphi}^{(2)} ; \boldsymbol{\psi}^{(2)} ; \delta \boldsymbol{\alpha}\right)=\sum_{i_{2}=1}^{T P} R_{i_{1} i_{2}}^{(2)}\left(\boldsymbol{\alpha}, \boldsymbol{\varphi}^{(2)} ; \boldsymbol{\psi}_{1, i_{1}}^{(2)}, \boldsymbol{\psi}_{i, i_{1}}^{(2)}\right) \delta \alpha_{i_{2}}, i_{1}=1, \ldots, T P,
$$

where the arguments in $\delta R_{i_{1}}^{(1)}\left(\boldsymbol{\alpha}, \boldsymbol{\varphi}^{(2)} ; \boldsymbol{\psi}^{(2)} ; \delta \boldsymbol{\alpha}\right)$ indicate that this quantity no longer depends on the variations $\delta \mathbf{u}$ and $\delta \psi^{(1)}$ but depends on the 2nd-level adjoint function $\psi^{(2)}$, and where

$$
\begin{aligned}
& R_{i_{1} i_{2}}^{(2)}\left(\boldsymbol{\alpha}, \boldsymbol{\varphi}^{(2)} ; \boldsymbol{\psi}_{1, i_{1}}^{(2)}, \boldsymbol{\psi}_{i, i_{1}}^{(2)}\right) \triangleq \frac{\partial}{\partial \alpha_{i_{2}}}\left[R_{i_{1}}^{(1)}\left(\boldsymbol{\alpha}, \boldsymbol{\varphi}^{(2)}\right)-\hat{P}^{(2)}\left(\boldsymbol{\alpha}, \boldsymbol{\varphi}^{(2)} ; \boldsymbol{\psi}_{1, i_{1}}^{(2)}, \boldsymbol{\psi}_{2, i_{1}}^{(2)}\right)\right] \\
& \quad+\int_{\Omega_{x}}\left\{\boldsymbol{\psi}_{1, i_{1}}^{(2)}(\mathbf{x}) \cdot\left[\frac{\partial\left[\Theta^{(1)}(\boldsymbol{\alpha}, \mathbf{u})-\mathbf{A}_{11}^{(1)}(\boldsymbol{\alpha}, \mathbf{u}) \boldsymbol{\psi}^{(1)}\right]}{\partial \alpha_{i_{2}}}\right]+\boldsymbol{\psi}_{2, i_{1}}^{(2)}(\mathbf{x}) \cdot\left[\frac{\partial[\mathbf{Q}(\boldsymbol{\alpha})-\mathbf{N}(\boldsymbol{\alpha}, \mathbf{u})]}{\partial \alpha_{i_{2}}}\right]\right\} d \mathbf{x} .
\end{aligned}
$$


As Equation (A53) indicates, each of the G-differentials $\delta R_{i_{1}}^{(1)}\left(\alpha, \varphi^{(2)} ; \psi^{(2)} ; \delta \alpha\right)$ contains one complete row (running on the index $i_{2}=1, \ldots, T P$ ) of the mixed second-order partial sensitivities, $R_{i_{1} i_{2}}^{(2)}\left(\boldsymbol{\alpha}, \boldsymbol{\varphi}^{(2)} ; \boldsymbol{\psi}_{1, i_{1}}^{(2)}, \boldsymbol{\psi}_{i, i_{1}}^{(2)}\right)$, which can be computed exactly and efficiently after solving only once the 2nd-Level Adjoint Sensitivity System (2nd-LASS), namely Equations (A47) and (A43), to obtain the 2 nd-level adjoint function $\boldsymbol{\psi}_{i_{1}}^{(2)}(\mathbf{x}) \triangleq\left(\boldsymbol{\psi}_{1, i_{1}}^{(2)}(\mathbf{x}), \boldsymbol{\psi}_{2, i_{1}}^{(2)}(\mathbf{x})\right) \in H_{\psi}^{(2)}\left(\Omega_{x}\right)$, for each index $i_{1}=1, \ldots, T P$.

Using in Equation (A47) the relations given in Equation (A48) indicates that the 2nd-LASS has the following form:

$$
\begin{gathered}
\mathbf{F}_{11}^{(1)}(\boldsymbol{\alpha}, \mathbf{u}) \boldsymbol{\Psi}_{1, i_{1}}^{(2)}(\mathbf{x})=\boldsymbol{\Theta}_{1, i_{1}}^{(2)}\left(\boldsymbol{\alpha}, \boldsymbol{\varphi}^{(2)}\right), \mathbf{x} \in \Omega_{x}, i_{1}=1, \ldots, T P, \\
\mathbf{A}_{11}^{(1)}(\boldsymbol{\alpha}, \mathbf{u}) \boldsymbol{\Psi}_{2, i_{1}}^{(2)}(\mathbf{x})=\boldsymbol{\Theta}_{2, i_{1}}^{(2)}\left(\boldsymbol{\alpha}, \boldsymbol{\varphi}^{(2)}\right)-\mathbf{A}_{21}^{(2)}(\boldsymbol{\alpha}, \mathbf{u}) \boldsymbol{\psi}_{1, i_{1}}^{(2)} \mathbf{x} \in \Omega_{x}, i_{1}=1, \ldots, T P .
\end{gathered}
$$

Since Equation (A54) is linear in $\psi_{1, i_{1}}^{(2)}(\mathbf{x})$, solving it requires less computational effort than solving the original Equation (1), which is nonlinear in $\mathbf{u}(\mathbf{x})$. Furthermore, solving Equation (A55) to obtain $\psi_{2, i_{1}}^{(2)}(\mathbf{x})$ is equivalent to solving the 1st-LASS, i.e., Equation (A25), but with a different source term. Thus, the same computer program and "solvers" can be used for solving the 2nd-LASS as were already used for solving the 1st-LASS and for solving the original system, cf., Equations (1) and (2). Only the sources for the respective operator equations and boundary conditions for the 2nd-LASS will differ from those used to solve the 1st-LASS and the original system of equations. Consequently, the 2nd-LASS (which comprises Equations (A54), (A55) and the boundary conditions represented by Equation (A43)) is called the "second-level" rather than "second-order" adjoint sensitivity system, since the 2nd-LASS does not involve any explicit 2nd-order G-derivatives of the operators underlying the original system but involves the inversion of the same operators that needed to be inverted for solving the 1st-LASS and the original system of equations.

Note that the $2 n d-L A S S$ is independent of parameter variations $\delta \alpha$, as well as of the variations $\delta \mathbf{u}$ and $\delta \psi^{(1)}$. Thus, the exact computation of all of the partial second-order sensitivities, $\left\{\partial^{2} R(\boldsymbol{\alpha}, \mathbf{u}) / \partial \alpha_{j} \partial \alpha_{i}\right\}_{\left(\boldsymbol{\alpha}^{0}, \mathbf{u}^{0}\right)}, i, j=1, \ldots, T P$, requires at most TP large-scale (adjoint) computations using the $2 n d$-LASS, rather than $O\left(T P^{2}\right)$ large-scale computations as would be required by forward methods. Another important characteristic of using the $2 n d-L A S S$ is the flexibility it provides for prioritizing the computation of the 2nd-order sensitivities. Only the 2nd-order partial sensitivities of the response $R(\mathbf{e})$ which are deemed important need to be computed; the unimportant ones can be deliberately neglected while knowing the error incurred by neglecting them. The computation of the 2nd-order sensitivities should be based on the priority ranking of the relative magnitudes of the 1st-order sensitivities: the 2nd-order mixed G-derivatives (sensitivities) corresponding to the largest relative 1st-order response sensitivity should be computed first; the second largest relative 1st-order response sensitivity should be considered next, and so on. Computing 2nd-order partial sensitivities that correspond to vanishing 1st-order sensitivities may also be of interest, since vanishing 1st-order sensitivities may indicate critical points of the response in the phase-space of model parameters.

It is also important to note that if the 2nd-LASS is solved TP-times, the 2nd-order mixed sensitivities $R_{i_{1} i_{2}}^{(2)}\left(\alpha, \varphi^{(2)} ; \psi_{1, i_{1}}^{(2)}, \psi_{2, i_{1}}^{(2)}\right)$ will be computed twice, in two different ways, thereby providing an intrinsic (numerical) verification that the 2nd-Level adjoint functions $\psi_{1, i_{1}}^{(2)}(\mathbf{x})$ and $\psi_{2, i_{1}}^{(2)}(\mathbf{x})$ are computed accurately. Finally, the total 2nd-order G-differential, $\delta^{(2)} R\left(\boldsymbol{\alpha}^{0}, \mathbf{u}^{0} ; \delta \boldsymbol{\alpha}_{1}, \delta \boldsymbol{\alpha}_{2}\right)$, of the response $R(\mathbf{e})$ at $\mathbf{e}^{0}=\left(\boldsymbol{\alpha}^{0}, \mathbf{u}^{0}\right)$ in a neighborhood $\left(\mathbf{e}^{0}+\varepsilon \mathbf{h}\right)$ around $\mathbf{e}^{0}$ is given by

$$
\delta^{(2)} R\left(\boldsymbol{\alpha}^{0}, \mathbf{u}^{0} ; \delta \boldsymbol{\alpha}_{1}, \delta \boldsymbol{\alpha}_{2}\right)=\sum_{i_{1}=1}^{T P} \sum_{i_{2}=1}^{T P} R_{i_{1} i_{2}}^{(2)}\left(\boldsymbol{\alpha}^{0}, \boldsymbol{\varphi}^{(2,0)} ; \boldsymbol{\psi}_{1, i_{1}}^{(2,0)}, \boldsymbol{\psi}_{2, i_{1}}^{(2,0)}\right) \delta \alpha_{i_{1}} \delta \alpha_{i_{2}}
$$


As Equation (A56) indicates, the 2nd-order partial sensitivities are functionals of the form $R_{i_{1} i_{2}}^{(2)}\left(\alpha, \varphi^{(2)} ; \psi_{i_{1}}^{(2)}\right) \in \mathrm{H}_{\alpha} \oplus \mathrm{H}_{\psi}^{(2)}\left(\Omega_{x}\right) \oplus \mathrm{H}_{\psi}^{(2)}\left(\Omega_{x}\right)=\mathrm{H}_{\alpha} \oplus 2 \otimes \mathrm{H}_{\psi}^{(2)}\left(\Omega_{x}\right) \rightarrow \mathrm{F}$.

\section{References}

1. Bellman, R.E. Dynamic Programming; Princeton University Press: Princeton, NJ, USA, 1957; ISBN1 978-0-691-07951-6. Courier Dover Publications: Mineola, NY, USA, 2003; ISBN2 978-0-486-42809-3.

2. Wigner, E.P. Effect of Small Perturbations on Pile Period; Chicago Report CP-G-3048; Springer: Berlin/Heidelberg, Germany, 1945.

3. Cacuci, D.G. Sensitivity theory for nonlinear systems: I. Nonlinear functional analysis approach. J. Math. Phys. 1981, 22, 2794-2802. [CrossRef]

4. Cacuci, D.G. Sensitivity theory for nonlinear systems: II. Extensions to additional classes of responses. J. Math. Phys. 1981, 22, 2803-2812. [CrossRef]

5. Mitani, H. Higher-order perturbation method in reactor calculation. Nucl. Sci. Eng. 1973, 51, 180-188. [CrossRef]

6. Seki, Y. Evaluation of the second-order perturbation terms by the generalized perturbation method. Nucl. Sci. Eng. 1973, 51, 243-251. [CrossRef]

7. Gandini, A. Implicit and explicit higher-order perturbation methods for nuclear reactor analysis. Nucl. Sci. Eng. 1978, 67, 347-355. [CrossRef]

8. Greenspan, E.; Gilai, D.; Oblow, E.M. Second-order generalized perturbation theory for source-driven systems. Nucl. Sci. Eng. 1978, 68, 1-9. [CrossRef]

9. Cacuci, D.G.; Weber, C.F.; Oblow, E.M.; Marable, J.H. Sensitivity Theory for General Systems of Nonlinear Equations. Nucl. Sci. Eng. 1980, 75, 88-110. [CrossRef]

10. Haug, E.J. Second-order design sensitivity analysis of structural systems. AIAA J. 1981, 19, 1087-1088. [CrossRef]

11. Haftka, R.T. Second-order sensitivity derivatives in structural analysis. AIAA J. 1982, 20, 1765-1766. [CrossRef]

12. Dems, K.; Mroz, Z. Variational approach to first- and second-order sensitivity analysis of elastic structures. Int. J. Numer. Methods Eng. 1985, 21, 637-661. [CrossRef]

13. Haug, E.J.; Ehle, P.E. Second-order design sensitivity analysis of mechanical system dynamics. Int. J. Numer. Methods Eng. 1982, 18, 1699-1717. [CrossRef]

14. Haftka, R.T.; Mroz, Z. First-and second-order sensitivity analysis of linear and nonlinear structures. AIAA J. 1986, $24,1187-1192$. [CrossRef]

15. Haug, E.; Komkov, J.V.; Choi, K.K. Design Sensitivity Analysis of Structural Systems; Academic Press: New York, NY, USA, 1986.

16. Ye, X.; Li, P.; Liu, F.Y. Exact time-domain second-order adjoint-sensitivity computation for linear circuit analysis and optimization. IEEE Trans. Circuits Syst. 2010, 57, 236-248.

17. Negm, M.H.; Bakr, M.H.; Ahmed, O.S.; Nikolova, N.K.; Bandler, J.W. Wideband second-order adjoint sensitivity analysis exploiting TLM. IEEE Trans. Microw. Theory Tech. 2014, 62, 389-398. [CrossRef]

18. Wang, Z.; Navon, I.M.; Le Dimet, F.X.; Zou, X. The second order adjoint analysis: Theory and applications. Meteorol. Atmos. Phys. 1992, 50, 3-20. [CrossRef]

19. Cacuci, D.G.; Ionescu-Bujor, M.; Navon, M.I. Sensitivity and Uncertainty Analysis: Applications to Large Scale Systems; Chapman \& Hall/CRC: Boca Raton, FL, USA, 2005; Volume 2.

20. Cacuci, D.G.; Navon, M.I.; Ionescu-Bujor, M. Computational Methods for Data Evaluation and Assimilation; Chapman \& Hall/CRC: Boca Raton, FL, USA, 2014.

21. Sandu, A.; Zhang, L. Discrete second order adjoints in atmospheric chemical transport modeling. J. Comput. Phys. 2008, 227, 5949-5983. [CrossRef]

22. Práger, T.; Kelemen, F.D. Adjoint methods and their application in earth sciences. In Advanced Numerical Methods for Complex Environmental Models: Needs and Availability; Faragó, I., Havasi, Á., Zlatev, Z., Eds.; Bentham Science Publishers: Sharjah, United Arab Emirates, 2014; pp. 203-275, Chapter 4A.

23. Cacuci, D.G. Second-Order Adjoint Sensitivity Analysis Methodology for Computing Exactly and Efficiently First- and SecondOrder Sensitivities in Large-Scale Linear Systems: I. Computational Methodology. J. Comp. Phys. 2015, 284, 687-699. [CrossRef]

24. Cacuci, D.G. Second-order adjoint sensitivity analysis methodology (2nd-ASAM) for large-scale nonlinear systems: I. Theory. Nucl. Sci. Eng. 2016, 184, 16-30. [CrossRef]

25. Cacuci, D.G. The Second-Order Adjoint Sensitivity Analysis Methodology; CRC Press, Taylor \& Francis Group: Boca Raton, FL, USA, 2018.

26. Cacuci, D.G.; Fang, R.; Favorite, J.A. Comprehensive second-order adjoint sensitivity analysis methodology (2nd-ASAM) applied to a subcritical experimental reactor physics benchmark: I. Effects of imprecisely known microscopic total and capture cross sections. Energies 2019, 12, 4219. [CrossRef]

27. Fang, R.; Cacuci, D.G. Comprehensive second-order adjoint sensitivity analysis methodology (2nd-ASAM) applied to a subcritical experimental reactor physics benchmark: II. Effects of imprecisely known microscopic scattering cross sections. Energies 2019, 12, 4114. [CrossRef] 
28. Cacuci, D.G.; Fang, R.; Favorite, J.A.; Badea, M.C.; Di Rocco, F. Comprehensive Second-Order Adjoint Sensitivity Analysis Methodology (2nd-ASAM) Applied to a Subcritical Experimental Reactor Physics Benchmark: III. Effects of Imprecisely Known Microscopic Fission Cross Sections and Average Number of Neutrons per Fission. Energies 2019, 12, 4100. [CrossRef]

29. Fang, R.; Cacuci, D.G. Comprehensive second-order adjoint sensitivity analysis methodology (2nd-ASAM) applied to a subcritical experimental reactor physics benchmark: IV. Effects of imprecisely known source parameters. Energies 2020, 13, 1431. [CrossRef]

30. Fang, R.; Cacuci, D.G. Comprehensive second-order adjoint sensitivity analysis methodology (2nd-ASAM) applied to a subcritical experimental reactor physics benchmark: V. Computation of mixed 2nd-order sensitivities involving isotopic number densities. Energies 2020, 13, 2580. [CrossRef]

31. Cacuci, D.G.; Fang, R.; Favorite, J.A. Comprehensive second-order adjoint sensitivity analysis methodology (2nd-ASAM) applied to a subcritical experimental reactor physics benchmark: VI. Overall impact of 1st- and 2nd-order sensitivities on response uncertainties. Energies 2020, 13, 1674. [CrossRef]

32. Valentine, T.E. Polyethylene-Reflected Plutonium Metal Sphere Subcritical Noise Measurements, SUB-PU-METMIXED-001. International Handbook of Evaluated Criticality Safety Benchmark Experiments; NEA/NSC/DOC(95)03/I-IX; Organization for Economic CoOperation and Development, Nuclear Energy Agency: Paris, France, 2006.

33. Alcouffe, R.E.; Baker, R.S.; Dahl, J.A.; Turner, S.A.; Ward, R. PARTISN: A Time-Dependent, Parallel Neutral Particle Transport Code System; LA-UR-08-07258; Los Alamos National Lab: Los Alamos, NM, USA, 2008.

34. Cacuci, D.G. Towards Overcoming the Curse of Dimensionality: The Third-Order Adjoint Method for Sensitivity Analysis of Response-Coupled Linear Forward/Adjoint Systems, with Applications to Uncertainty Quantification and Predictive Modeling. Energies 2019, 12, 4216. [CrossRef]

35. Cacuci, D.G. Nuclear Thermal-Hydraulics Applications Illustrating the Key Roles of Adjoint-Computed Sensitivities for Overcoming the Curse of Dimensionality in Sensitivity Analysis, Uncertainty Quantification and Predictive Modeling. Nucl. Eng. Des. 2019, 351, 20-32. [CrossRef]

36. Cacuci, D.G.; Fang, R. Third-Order Adjoint Sensitivity Analysis of an OECD/NEA Reactor Physics Benchmark: I. Mathematical Framework. Am. J. Comput. Math. 2020, 10, 503-528. [CrossRef]

37. Fang, R.; Cacuci, D.G. Third-Order Adjoint Sensitivity Analysis of an OECD/NEA Reactor Physics Benchmark: II. Computed Sensitivities. Am. J. Comput. Math. 2020, 10, 529-558. [CrossRef]

38. Fang, R.; Cacuci, D.G. Third-Order Adjoint Sensitivity Analysis of an OECD/NEA Reactor Physics Benchmark: III. Response Moments. Am. J. Comput. Math. 2020, 10, 559-570. [CrossRef]

39. Cacuci, D.G. Fourth-Order Comprehensive Adjoint Sensitivity Analysis (4th-CASAM) of Response-Coupled Linear Forward/Adjoint Systems. I. Theoretical Framework. Energies 2021, 14, 3335. [CrossRef]

40. Cacuci, D.G.; Fang, R. Fourth-Order Adjoint Sensitivity Analysis of an OECD/NEA Reactor Physics Benchmark: I. Mathematical Expressions and CPU-Time Comparisons for Computing 1st-, 2nd- and 3rd-Order Sensitivities. Am. J. Comput. Math. 2021, 2, 94-132. [CrossRef]

41. Cacuci, D.G.; Fang, R. Fourth-Order Adjoint Sensitivity Analysis of an OECD/NEA Reactor Physics Benchmark: II. Mathematical Expressions and CPU-Time Comparisons for Computing 4th-Order Sensitivities. Am. J. Comput. Math. 2021, 11, 133-156. [CrossRef]

42. Lewins, J. Importance: The Adjoint Function; Pergamon Press: Oxford, UK, 1965.

43. Stacey, W.M., Jr. Variational Methods in Nuclear Reactor Physics; Academic Pres: New York, NY, USA, 1974.

44. Stacey, W.M. Nuclear Reactor Physics; John Wiley \& Sons Inc.: New York, NY, USA, 2001.

45. Bethe, H. Nuclear Physics, B, Nuclear Dynamics, Theoretical. Rev. Mod. Phys. 1937, 9, 121. [CrossRef]

46. Weiberg, A.M.; Wigner, E.P. The Physical Theory of Neutron Chain Reactors; University of Chicago Press: Chicago, IL, USA, 1958.

47. Amaldi, E. The Production and Slowing Down of Neutrons. In Handbuch der Physik; Part 2; Springer: Berlin/Heidelberg, Germany, 1959; Volume 38, pp. 1-659.

48. Meghreblian, R.V.; Holmes, D.K. Reactor Analysis; McGraw-Hill Book Co.: New York, NY, USA, 1960.

49. Tukey, J.W. The Propagation of Errors, Fluctuations and Tolerances; Unpublished Technical Reports No. 10-12; Princeton University: Princeton, NJ, USA, 1957.

50. Cacuci, D.G. Sensitivity and Uncertainty Analysis: Theory; Chapman \& Hall/CRC: Boca Raton, FL, USA, 2003 ; Volume 1. 